\title{
A Measurement of the Top Quark Mass with a Matrix Element Method
}

by

\author{
Adam Paul Gibson
}

B.A. (Valparaiso University, Indiana) 2000

M.A. (University of California, Berkeley) 2002

A dissertation submitted in partial satisfaction of the
requirements for the degree of
Doctor of Philosophy
in
Physics
in the
GRADUATE DIVISION
of the
UNIVERSITY OF CALIFORNIA, BERKELEY

Committee in charge:

Professor Young-Kee Kim, Co-Chair

Professor James Siegrist, Co-Chair

Professor Yury Kolomensky

Professor Chung-Pei Ma

Fall 2006 
A Measurement of the Top Quark Mass with a Matrix Element Method

Copyright 2006

by

Adam Paul Gibson 


\begin{abstract}
A Measurement of the Top Quark Mass with a Matrix Element Method

by

Adam Paul Gibson

Doctor of Philosophy in Physics

University of California, Berkeley

Professor Young-Kee Kim, Co-Chair

Professor James Siegrist, Co-Chair
\end{abstract}

We present a measurement of the mass of the top quark. Our event sample is selected from proton-antiproton collisions, at $1.96 \mathrm{TeV}$ center-of-mass energy, observed with the CDF detector at Fermilab's Tevatron. We consider a $318 \mathrm{pb}^{-1}$ dataset collected between March 2002 and August 2004. We select events that contain one energetic lepton, large missing transverse energy, exactly four energetic jets, and at least one displaced vertex $b$ tag. Our analysis uses leading-order $t \bar{t}$ and background matrix elements along with parameterized parton showering to construct event-by-event likelihoods as a function of top quark mass. From the 63 events observed with the $318 \mathrm{pb}^{-1}$ dataset we extract a top quark mass of $172.0 \pm 2.6$ (stat) \pm 3.3 (syst) $\mathrm{GeV} / c^{2}$ from the joint likelihood. The mean expected statistical uncertainty is $3.2 \mathrm{GeV} / c^{2}$ for $m_{t}=178 \mathrm{GeV} / c^{2}$ and $3.1 \mathrm{GeV} / c^{2}$ for $m_{t}=172.5$ $\mathrm{GeV} / c^{2}$. The systematic error is dominated by the uncertainty of the jet energy scale.

Professor Young-Kee Kim Dissertation Committee Co-Chair

Professor James Siegrist Dissertation Committee Co-Chair 
To my incomparable wife Erica, and my new son Daniel. 


\section{Contents}

List of Figures $\quad$ v

List of Tables $\quad$ ix

1 Introduction 1

2 Motivation and Theoretical Context 5

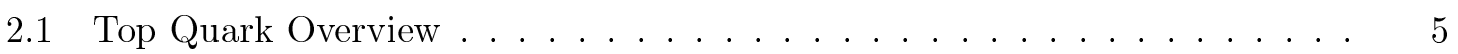

2.1 .1 Top Production . . . . . . . . . . . . . . . . . . 6

2.1 .2 Top Decays . . . . . . . . . . . . . . . . . . . 10

2.2 Precision Electroweak Physics . . . . . . . . . . . . . . . . . . . 12

2.3 Top Quark Beyond the Standard Model . . . . . . . . . . . . . . . . 16

2.4 Other Top Mass Measurements . . . . . . . . . . . . . . . . . . . . . . 19

2.4 Template Methods . . . . . . . . . . . . . . . . 20

2.4.2 Matrix Element Methods . . . . . . . . . . . . . . . . 22

2.4 .3 Recent Measurements . . . . . . . . . . . . . . . 23

3 Experimental Apparatus $\quad 29$

3.1 Accelerator Complex . . . . . . . . . . . . . . . . . . . . 29

3.1.1 Proton Source, Linac, and Booster . . . . . . . . . . . . . 29

3.1 .2 Main Injector . . . . . . . . . . . . . . . . . . . . . . 31

3.1.3 Antiproton Production and Storage . . . . . . . . . . . 31

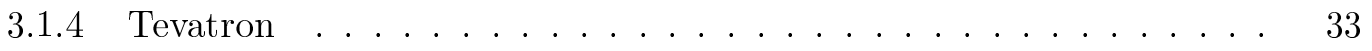

3.2 Collider Detector at Fermilab . . . . . . . . . . . . . . . . 35

3.2 .1 Silicon Vertex Detector . . . . . . . . . . . . . 36

3.2.2 Central Outer Tracker: COT . . . . . . . . . . . . . . . 39

3.2 .3 Calorimeters . . . . . . . . . . . . . . . . . . 41

3.2 .4 Muon Detectors . . . . . . . . . . . . . . . . . . . 43

3.2.5 Cherenkov Luminosity Counter . . . . . . . . . . . . . . . . . 45

3.2.6 Data Acquisition and Trigger System . . . . . . . . . . . . 45

3.2.7 Monte Carlo Event Generation and Detector Simulation . . . . . . . 48 
4 Selection and Reconstruction of $t \bar{t}$ Events $\quad \mathbf{5 0}$

$4.1 t \bar{t}$ Event Characteristics . . . . . . . . . . . . . . . . . . 50

4.2 Trigger Requirements . . . . . . . . . . . . . . . . . 52

4.2.1 Triggering Electrons . . . . . . . . . . . . . . . 52

4.2 .2 Triggering Muons . . . . . . . . . . . . . . . . . . 53

4.3 Offline Event Reconstruction _. . . . . . . . . . . . . . 53

4.3.1 Offline Electron Reconstruction and Selection . . . . . . . . . . 54

4.3.2 Offline Muon Reconstruction and Selection . . . . . . . . . 55

4.4 Jet Reconstruction and Corrections . . . . . . . . . . . . . . 55

4.4 .1 Jet Clustering . . . . . . . . . . . . . . . . 56

4.4 .2 Jet Energy Corrections . . . . . . . . . . . . . . . . . . 57

4.4 .3 Displaced Vertex $b$-Tagging . . . . . . . . . . . . . . . 60

4.5 Missing Transverse Energy . . . . . . . . . . . . . . . . . . . . . . 61

4.6 Summary of Event Selection Requirements . . . . . . . . . . . . . . 62

4.7 Events Observed in Data . . . . . . . . . . . . . . . . . . 62

4.8 Background Estimates and Modelling . . . . . . . . . . . . . . 63

$4.8 .1 W+$ Jets . . . . . . . . . . . . . . . 67

4.8.2 Electroweak and Single Top . . . . . . . . . . . . . . . 70

4.8 .3 Non-W QCD . . . . . . . . . . . . . . . . . . . . 71

4.8.4 Systematic Error on Background Estimates . . . . . . . . . . . 72

5 General Analysis Method and Likelihood Formalism $\quad 74$

$\begin{array}{llr}6 & \text { Transfer Functions } & \mathbf{7 9}\end{array}$

6.1 Jet Energy Transfer Functions _ . . . . . . . . . . . . . . . . . . . 79

6.2 Tests and Cross-Checks . . . . . . . . . . . . . . . 81

6.2.1 One-Dimensional Checks . . . . . . . . . . . . . . . . 82

6.2.2 Two-jet and Three-jet Invariant Masses . . . . . . . . . . . . . . . . . 84

6.3 Systematic Studies . . . . . . . . . . . . . . . . . . . . . 91

6.4 Comparison of Transfer Functions in Different Analyses . . . . . . . . . . . 92

7 Signal Probability $\quad 94$

7.1 Signal Probability in Analytic Form . . . . . . . . . . . . . . . . . . 94

7.2 Exploring the Signal Probability . . . . . . . . . . . . . . . . 100

7.3 Additional Event Selection . . . . . . . . . . . . . . . . . . 102

7.4 Pseudo-Experiment Methodology . . . . . . . . . . . . . . . 106

7.5 Tests of Signal-Only Likelihood with $t \bar{t}$ Pseudo-

Experiments . . . . . . . . . . . . . . . . . . 107

7.6 Tests of Signal-Only Likelihood with

Signal + Background Pseudo-Experiments . . . . . . . . . . . . . . . . 109

8 Background Probability $\quad 112$

8.1 ALPGEN . . . . . . . . . . . . . . . . . . . . . 113

8.2 Interface to ALPGEN . . . . . . . . . . . . . . . . . . . . . . . . . . . . . . . . . . .

8.3 ALPGEN $W q q j j$ process $\ldots \ldots \ldots \ldots \ldots$ 
8.3.1 Wqqjj: differential cross section . . . . . . . . . . . . . . 116

8.3 .2 Performance . . . . . . . . . . . . . . . . . 117

8.3.3 Normalization . . . . . . . . . . . . . . . . . . . . . . 120

8.4 W+Jets Probability using Leading Order VECBOS Matrix Element . . . . 120

8.5 ALPGEN - VECBOS comparison . . . . . . . . . . . . . . . . 124

9 Tests of Signal + Background Likelihood 127

9.1 Pseudo-Experiments for $\sigma_{t \bar{t}}=6.1 \mathrm{pb} \ldots \ldots \ldots \ldots \ldots \ldots$

9.2 Pseudo-Experiments for 63-Event Samples . . . . . . . . . . . . . 133

10 Expected Error and Results from Data 138

10.1 Expected Error . . . . . . . . . . . . . . . . . 138

10.2 Results from Data . . . . . . . . . . . . . . . . . . . 140

11 Systematic Uncertainty 142

11.1 Jet Energy Scale . . . . . . . . . . . . . . . . . . . . . 143

11.2 b Jet Energy Scale . . . . . . . . . . . . . . . . . . . . . . 145

11.3 Generator . . . . . . . . . . . . . . . . . . 145

11.4 Radiation . . . . . . . . . . . . . . . . . . . . . 146

11.5 $t \bar{t}$ Production via Gluon Fusion ( $g g)$ vs. Quark Anti-Quark Annihilation ( $q q) 147$

11.6 Uncertainties in Parton Density Functions . . . . . . . . . . . . . . 148

11.7 Background Composition _. . . . . . . . . . . . . . 150

11.8 Background Modelling . . . . . . . . . . . . . . . . . . 151

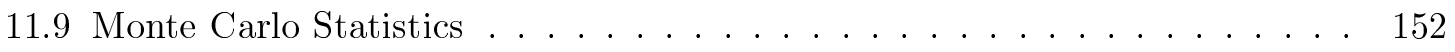

11.10Uncertainty on Mapping Function . . . . . . . . . . . . . . . . . 154

$11.11 b$ Tagging Scale Factor Uncertainty . . . . . . . . . . . . . . . . . . . . 154

11.12Summary of Systematic Errors _. . . . . . . . . . . . . . 154

12 Additional Checks 156

12.1 Understanding the Widths of Pull Distributions . . . . . . . . . . . . . 156

12.2 Results of Blind Samples . . . . . . . . . . . . . . . 166

13 Conclusions $\quad 168$

$\begin{array}{ll}\text { Bibliography } & 172\end{array}$ 


\section{List of Figures}

2.1 Feynman diagrams for $t \bar{t}$ production via the strong interaction, as at a hadron

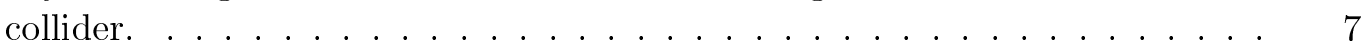

2.2 Latest $t \bar{t}$ production cross section measurements from CDF . . . . . . . 8

$2.3 \sqrt{s}$ dependence of $t \bar{t}$ cross section at CDF $\ldots \ldots \ldots \ldots$

$2.4 m_{t}$ dependence of $t \bar{t}$ cross section at CDF Run $2 \ldots \ldots \ldots \ldots$

2.5 Feynman diagrams for single-top production . . . . . . . . . . . . . . . 10

2.6 Feynman diagram for $t \bar{t}$ production and decay . . . . . . . . . . . . . . 11

2.7 One-loop contributions from the top quark to the $W$ and $Z$ masses. . . . . 13

2.8 One-loop contributions from the Higgs to the $W$ and $Z$ masses. . . . . . . . 14

$2.9 M_{W}$ vs. $m_{t}$ with lines of constant Higgs mass. Direct measurements compared to predictions from precision electroweak. . . . . . . . . . 15

2.10 The impact of current and future $m_{t}$ and $M_{W}$ measurements in the Standard Model and MSSM ........................ 18

2.11 Summary of Tevatron $M_{\text {top }}$ measurements, with preliminary average . . . . 20

2.12 Summary of Run $2 m_{t}$ measurements at CDF as of July 2005 . . . . . . . . 21

2.13 Summary of Run $2 m_{t}$ measurements at CDF as of March $2006 \ldots \ldots$

2.14 Projections for CDF Top Mass Uncertainty in Run $2 \ldots$. . . . . . . . . 27

3.1 Accelerator Chain . . . . . . . . . . . . . . . . . 30

3.2 Tevatron performance . . . . . . . . . . . . . . 34

3.3 Cartoon of particle interactions in detector. . . . . . . . . . . 36

3.4 Elevation View of CDF Detector . . . . . . . . . . . . . . 37

3.5 CDF Tracking System . . . . . . . . . . . . . . . . . . . . 38

3.6 CDF Silicon System . . . . . . . . . . . . . . . . . . 38

3.7 Central Outer Tracker Endplate and Cell Layout . . . . . . . . . . . . . 40

3.8 Plug Calorimeter . . . . . . . . . . . . . . . . . . . . 41

3.9 Central Electromagnetic Calorimeter (CEM) Wedge . . . . . . . . . . . 43

$3.10 \eta-\phi$ Coverage of Muon Detectors . . . . . . . . . . . . . . . . . . . 44

3.11 Muon Detector Depths . . . . . . . . . . . . . . . . . . 45

3.12 Trigger electronics schematic . . . . . . . . . . . . . 46

3.13 Progression of physics content through the Level 1 and Level 2 triggers . . . 47

4.1 Feynman diagram for $t \bar{t}$ production and decay . . . . . . . . . . . . . 51 
4.2 Di-jet balancing for data and $\mathrm{MC} \ldots \ldots \ldots \ldots \ldots$

4.3 Jet energy correction due to multiple interactions . . . . . . . . . . . 59

4.4 Absolute jet energy correction . . . . . . . . . . . . . . . 59

4.5 SecVtx efficiency as a function of jet $E_{T}$ and $\eta \ldots \ldots \ldots$. . . . . . 61

$5.1 t \bar{t}$ acceptance for baseline event selection . . . . . . . . . . . . 77

$5.2 t \bar{t}$ acceptance for $P_{t \bar{t}}$ cut $\ldots \ldots \ldots \ldots \ldots \ldots \ldots$

5.3 Overall $t \bar{t}$ acceptance . . . . . . . . . . . . . . . . . . 78

6.1 Light quark parton energy distribution with fit . . . . . . . . . . 83

$6.2 b$ quark parton energy distribution with fit . . . . . . . . . . . . . 84

6.3 $E_{\text {parton }}-E_{\text {jet }}$ for light jets, comparison between Monte Carlo and predictions from transfer function . . . . . . . . . . . . . . . 85

$6.4 E_{\text {parton }}-E_{\text {jet }}$ for $b$ jets, comparison between Monte Carlo and predictions from transfer function . . . . . . . . . . . . . . . 86

6.5 Two- and three-jet invariant mass comparison of fully simulated events to predictions from transfer functions, correct combination . . . . . . . . .

6.6 Two- and three-jet invariant mass comparison of fully simulated events to predictions from transfer functions, all 12 combinations . . . . . . . 89

6.7 Two- and three-jet invariant mass comparison of fully simulated events to predictions from transfer functions, no matching requirement . . . . . . 90

$7.1 P_{t \bar{t}}$ for twenty fully simulated $t \bar{t}$ events $\ldots \ldots \ldots \ldots \ldots 1 . \ldots \ldots 1$

7.2 Close-up of $P_{t \bar{t}}$ for two fully simulated $t \bar{t}$ events . . . . . . . . . . . . . . . 101

7.3 Negative log likelihood curve for ensemble of twenty $t \bar{t}$ events . . . . . . . . 102

$7.4 P_{t \bar{t}}$ distribution for a pathological event . . . . . . . . . . . . 103

7.5 Mean $m_{t}$ values of pseudo-experiments drawn from a sample with, and without, a single pathological $t \bar{t}$ event . . . . . . . . . . . . . . . . . . . . 104

7.6 Means and errors from 35.94 event $t \bar{t}$ pseudo-experiments analyzed with signal-only likelihood . . . . . . . . . . . . . . . . . . 108

7.7 Pulls from 35.94 event $t \bar{t}$ pseudo-experiments analyzed with signal-only likelihood . . . . . . . . . . . . . . . . . . . . . . . 108

7.8 Linearity tests with $t \bar{t}$ pseudo-experiments analyzed with signal-only likelihood 109

7.9 Pull widths as a function of $m_{t}$ for $t \bar{t}$ pseudo-experiments with signal-only likelihood . . . . . . . . . . . . . . . . . . 110

7.10 Linearity tests with $t \bar{t}+$ background pseudo-experiments analyzed with signalonly likelihood . . . . . . . . . . . . . . . . . 110

7.11 Pull widths as a function of $m_{t}$ for $t \bar{t}+$ background pseudo-experiments analyzed with signal-only likelihood . . . . . . . . . . . .

8.1 Variation in $\log \left(|M|^{2}\right)$ calculated with ALPGEN with an increasing number

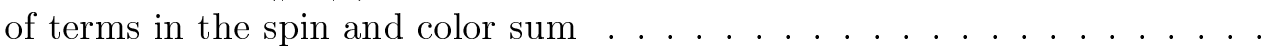

8.2 Variation of the squared ALPGEN matrix element for $p p \rightarrow W(\rightarrow l<n u)+$ $q q+j j$ as a function of the $l \nu$ invariant mass for a specific event. $\ldots . . .115$ 
8.3 ALPGEN background probability for parton level and Gaussian smeared parton level $t \bar{t}$ and $W b \bar{b}$ events . . . . . . . . . . . . .

8.4 ALPGEN background probability for fully simulated $t \bar{t}, W+4 p$, and $W b \bar{b}$ events. . . . . . . . . . . . . . . . . . . 119

8.5 Prospects for ALPGEN background probability as an event selection variable 119

8.6 VECBOS background probability distribution for fully simulated $W+$ jets, non- $W$ QCD and $t \bar{t}$ events. . . . . . . . . . . . . . . . . . . 123

8.7 Fully simulated HERWIG $178 \mathrm{GeV} / \mathrm{c}^{2}$ (ttophl) and ALPGEN+HERWIG $W+4$ parton and $W b \bar{b}$ samples processed with the VECBOS background probability. We plot the log of the differential cross section. . . . . . . . . . 123

8.8 Prospects for VEBCOS background probability as an event selection variable. 124

8.9 Log of ALPGEN probability vs. log of VECBOS probability for fully simulated $t \bar{t}$ (left) and $W+4$ parton, with the $W$ decaying to an electron (right), events. . . . . . . . . . . . . . . .

8.10 Log of ALPGEN probability vs. log of VECBOS probability for fully simulated $W+4$ parton events with the $W$ decaying to an electron (left) and $W b \bar{b}$ events with the $W$ decaying to a muon (right). . . . . . . . . . .

8.11 Log of ALPGEN probability vs. log of VECBOS probability for fully simulated $W b \bar{b}$ events where the $W$ decays to an electron. . . . . . . . . . . . 126

9.1 Linearity tests with $t \bar{t}+$ background pseudo-experiments $(6.1 \mathrm{pb})$ analyzed with full signal + background likelihood . . . . . . . . . . . . .

$9.2 m_{t}$ residuals with $t \bar{t}+$ background pseudo-experiments $(6.1 \mathrm{pb})$ analyzed with full likelihood . . . . . . . . . . . . . . . .

9.3 Pull widths as a function of $m_{t}$ for $t \bar{t}+$ background pseudo-experiments (6.1 pb) with full likelihood . . . . . . . . . . . . . . .

9.4 Linearity tests with $t \bar{t}+$ background pseudo-experiments $(6.1 \mathrm{pb})$ analyzed with full signal + background likelihood and mapping function . . . . . . .

9.5 Pull widths with $t \bar{t}+$ background pseudo-experiments $(6.1 \mathrm{pb})$ analyzed with full signal + background likelihood and mapping function . . . . . . . . .

9.6 Pull means with $t \bar{t}+$ background pseudo-experiments $(6.1 \mathrm{pb})$ analyzed with full signal + background likelihood and mapping function . . . . . . . . .

9.7 Linearity tests with $t \bar{t}+$ background pseudo-experiments (63 events) analyzed with full signal + background likelihood . . . . . . . . . . . . .

$9.8 m_{t}$ residuals with $t \bar{t}+$ background pseudo-experiments (63 events) analyzed with full likelihood . . . . . . . . . . . . . . .

9.9 Pull widths as a function of $m_{t}$ for $t \bar{t}+$ background pseudo-experiments (63 events) with full likelihood . . . . . . . . . . . . . . . .

9.10 Linearity tests with $t \bar{t}+$ background pseudo-experiments (63 events) analyzed with full signal + background likelihood and mapping function . . . .

9.11 Pull widths with $t \bar{t}+$ background pseudo-experiments (63 events) analyzed with full signal + background likelihood and mapping function . . . . . . .

9.12 Pull means with $t \bar{t}+$ background pseudo-experiments (63 events) analyzed with full signal + background likelihood and mapping function . . . . . . . 137

9.13 Expected error as a function of $m_{t}$ for 63 event pseudo-experiments. . . . . 137 
10.1 Error distribution for $6.1 \mathrm{pb}$ pseudo-experiments with $m_{t}=178 \mathrm{GeV} / c^{2}$. . 139

10.2 Error distribution for 63-event pseudo-experiments with $m_{t}=178 \mathrm{GeV} / c^{2} .139$

10.3 Error distribution for 63-event pseudo-experiments with $m_{t}=172.5 \mathrm{GeV} / c^{2}$, for comparison with measurement from collider data . . . . . . . . . . . . 140

10.4 Likelihood fit to the 63 events observed in $318 \mathrm{pb}^{-1}$ of data at CDF . . . . 141

10.5 Alternative views of the likelihood fit to the 63 events observed in $318 \mathrm{pb}^{-1}$ of data at $\mathrm{CDF} \ldots \ldots \ldots \ldots$. . . . . . . . . . . . . . . . 141

11.1 Comparison of $m_{t}$ results as a function of parton distribution function (PDF) for $46 \mathrm{PDF}$ sets including sets from CTEQ and MRST and the 40 different CTEQ6M eigenvector sets . . . . . . . . . . . . . . . . . . 149

11.2 Effect of the limited number of QCD events on estimates derived from pseudoexperiments . . . . . . . . . . . . . . . . . 153

$12.1 m_{t}$ results for blinded $t \bar{t}$ samples $\ldots \ldots \ldots \ldots \ldots$. . . . . . . . 167 


\section{List of Tables}

2.1 Current and anticipated experimental uncertainties for $M_{W}$ and $m_{t} \ldots \ldots$

3.1 Parameters for Several Tevatron Stores . . . . . . . . . . . . . . . . 34

$4.1 t \bar{t}$ electron candidate events observed in $318 \mathrm{pb}^{-1}$ of data collected from March 2002 to August 2004. . . . . . . . . . . . . . . . . . . .

$4.2 t \bar{t}$ muon candidate events observed in $318 \mathrm{pb}^{-1}$ of data collected from March 2002 to August 2004. . . . . . . . . . . . . . . . . 65

4.3 Estimated number of background events in our dataset. . . . . . . . . . 67

4.4 Systematic uncertainties on background estimates. . . . . . . . . 73

6.1 Default transfer function parameters . . . . . . . . . . . . . . 82

6.2 Parton energy distribution parameters corresponding to Eq. 6.6. . . . . . . 83

6.3 Summary of $m_{j j}$ and $m_{j j j}$ shifts in transfer function tests $\ldots \ldots \ldots$. . . 91

7.1 $t \bar{t}$ and background samples before and after cuts related to $P_{t \bar{t}} \ldots \ldots \ldots$

11.1 Systematic uncertainty due to the jet energy scale . . . . . . . . . . . . 144

11.2 Estimated systematic error due to differences in event generators and parton showering codes . . . . . . . . . . . . . . . 145

11.3 Estimated uncertainty due to initial- and final-state radiation . . . . . . . 146

11.4 Measured $m_{t}$ as a function of $g g$ fraction . . . . . . . . . . . . . . 147

11.5 Parton distribution function comparison for fully simulated samples . . . . 150

11.6 Systematic uncertainty due to background composition and normalization . 151

11.7 Systematic uncertainty due to background modelling. . . . . . . . . . . . . 152

11.8 Systematic uncertainty due to possible $E_{T}$-dependence of $b$ tag scale factor, the ratio of the number of $b$ tags expected in data and Monte Carlo . . . 155

11.9 Summary of Systematic Errors _ . . . . . . . . . . . . . . 155

12.1 Pull width studies for events close to parton level . . . . . . . . . . . . . 159

12.2 Pull width studies for various jet-related event selections . . . . . . . . . . 160

12.3 Widths of pull distributions for various lepton-related event selections . . . 160

12.4 Pull distributions for selected samples without the $P_{t \bar{t}}$ cut. . . . . . . . 161

12.5 Widths of pull distributions for various amounts of initial-state radiation $(t \bar{t}$

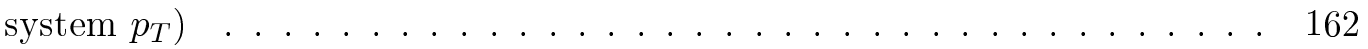


12.6 Widths of pull distributions for various types of lepton information . . . . . 162

12.7 Widths of pull distributions for various types of matching requirements . . 163 12.8 Widths of pull distributions in different bins of $p_{T}^{t \bar{t}}$ to check for radiation effects 163 12.9 Widths of pull distributions for events with four or more tight jets . . . . . 164 12.10Widths of pull distributions for various jet combinations . . . . . . . . . . 164 12.11 Summary of pull width studies . . . . . . . . . . . . 165 


\section{Acknowledgments}

An enormous number of people have contributed, directly and indirectly, to the measurement described in this dissertation and I am personally indebted to many of them.

Any measurement at a large and complex experiment like CDF relies on the efforts of hundreds of other individuals. This fact is partially reflected in the lengthy author lists of our journal articles. This measurement, of course, relies on the hard work of hundreds of CDF members (and non-members) in designing, building, calibrating, and maintaining the detector, and in cooperatively understanding the data that we collectively analyze. The top mass measurement reported in this dissertation relies specifically on the $t \bar{t}$ background estimates performed by Sal Rappoccio and colleagues for the top cross section measurement, and I would like to thank them for their efforts. Similarly, this measurement is patterned significantly after the Run I top mass measurement at D0 by Juan Estrada, Florencia Canelli, and Gaston Gutierrez. I am grateful for their example, and especially for Florencia's careful explanations of the techniques, and her advice throughout the course of this measurement. At CDF, I would also like to thank Daniel Whiteson, Erik Brubaker, Jason Nielsen, Andrew Kovalev, Brian Mohr, Kohei Yorita, Jeremy Lys, and Igor Volobouev for many useful conversations about this analysis.

Then, in a general fashion, I am indebted to many individuals, many of who will remain unnamed, for matters scientific, practical, and personal.

The person most responsible for guiding my graduate career is Young-Kee Kim.

I appreciate Young-Kee's guidance especially for the broad view it gave me of the CDF experiment and particle physics generally, and for her incomparable match-making services. She was repeatedly able to connect me with interesting projects and excellent groups of people to work with.

I learned a great deal, and made many friends, in my various efforts at CDF. This measurement would certainly not have been the same without my partners in crime in the MEAT (Matrix Element Analysis of the Top mass is one, non-canonical, explication) analysis group, especially Florencia, Daniel, Brian, and Eva. I would like to thank Kaori and Greg for their guidance in matters of YMon and online monitoring, and Beate, Anwar, and Giuseppe, among many others, for direction in the world of jet energy corrections. Erik, George, Guram, and Young-Kee were excellent companions for my introduction to top physics, in the guise of the template mass measurement, as later were Jean-Francois, 
Pekka, and Jahred.

I am very grateful for the excellent bunch of grad students, postdocs, and staff scientists in the LBL CDF group. They improved my life daily, in many ways. Thanks especially to Greg, Erik, Henri, Hung-Chung, Jason, and Weiming.

Many faculty members at Berkeley and at Valparaiso were instrumental in my development as a physicist. I especially appreciate the efforts of, and the example set by, Jim Siegrist and Marjorie Shapiro at Berkeley, and Don Koetke, Bob Manweiler, Shirvel Stanislaus, Stan Zygmunt, Bruce Hrivnak, and Dave Grosnick at Valparaiso. The set at Valparaiso, along with Todd Hillwig, Garry Morris, Andy Richter, and David Latimer, also made exceptional colleagues during my year of teaching, during the 2005-2006 academic year, at Valparaiso. I have very much appreciated administrative support at Berkeley and Valparaiso over the years. I am especially grateful for Anne Takizawa's support across three states and three countries over the last six years.

For their efforts in ensuring my psychological and spiritual survival during grad school, I would like to thank Bruce Tammen and the Chicago Chorale, Stephen Pordes and the Fermilab Singers, and three different Lutheran churches (Trinity Alameda, Geneva Lutheran, and St. Andrews Glenwood) and especially their musicians. For their contributions to my physical as well as psychological well-being (nothing beats chasing a piece of plastic for working out job-related frustrations) I would like to recognize all the folks who showed up for Fermilab Ultimate, especially Kip, Alon, Pete, Doug, Jim, and Satish, as well as the members of the Springfield Isotopes softball team, especially Mike, Dan, Pete, Ben, Robin, and John.

I will always be especially indebted, for their contributions throughout my life, and for their unending emotional and psychological support, to my family. I couldn't have done it without you!

Soli Deo Gloria. 


\section{Chapter 1}

\section{Introduction}

At least as far back as the 5th century BC, when the likes of Anaxagoras, Leucippus, and Democritus pondered atomic theory on Aegean shores, humans have wondered what the fundamental constituents of matter might be. Are there any fragments of material so small that they are, indeed, uncuttable? Democritus and other Greek philosophers were on to something when they argued that such an $\alpha \tau o \mu \circ \varsigma$ would have only a few properties - such as size, shape, and mass - and that all of the complex phenomena that we observe in nature are merely the result of the interactions of these fundamental $\alpha \tau o \mu o \iota$. Today we might prefer to substitute intrinsic spin and charge for Democritus' size and shape ${ }^{1}$, but we agree that mass is fundamental.

Beginning before the turn of the 20th century and, continuing beyond the turn of the 21st century, one of the most important pursuits of physicists has been to understand the elementary nature of matter. In 1897 J. J. Thomson confirmed the corpuscular nature of cathode rays by measuring their charge to mass ratio and identified them as universal constituents of matter [1]. The charge to mass ratio was the same regardless of the source of the cathode rays. So, Thomson had made the first concrete identification of what we now consider a fundamental particle, the electron.

Through the course of the 20th century a unified picture of elementary particles and their interactions began to appear. It included matter particles, the fermions, and

\footnotetext{
${ }^{1}$ Democritus would be amused to hear modern-day natural philosophers, such as particle physicists, discussing color and flavor as fundamental properties of matter. He might have explained a bitter flavor as originating from a combination of atoms whose shapes contained sharp corners. Presumably an alternate explanation would present itself for a strange or charmed flavor. He would have explained color also as a derived property rather than a fundamental one.
} 
particles responsible for their interactions, the gauge bosons. The fermions include three generations, nearly identical apart from their masses. Each generation contains two quarks, a charged lepton, and a neutral, elusive, neutrino. The bosons are the $W^{ \pm}$and $Z$, responsible for the weak interaction, the photon, responsible for the electromagnetic interaction, and the gluon, responsible for the strong interaction. This list of particles and their interactions are collectively contained in the Standard Model of particle physics. The Standard Model successfully describes the results of a tremendous number and variety of experiments involving elementary particles. The Standard Model does not describe the gravitational interaction, the origin of neutrino masses is not yet clear, and there are a variety of other reasons to suspect that the Standard Model is not the final theory of particle interactions. But, it remains a spectacularly successful description of nature.

The most recently confirmed entires to the Standard Model menu of particles are two of the third generation fermions, the tau neutrino and the top quark. The direct observation of the tau neutrino was reported in the year 2000 [2] while Fermilab has recently celebrated the tenth anniversary of the discovery of the top quark at the Collider Detector at Fermilab (CDF) $[3,4,5]$ and D0 [6]. Much remains yet to be learned about both the top quark and the tau neutrino, but one critical piece of the Standard Model yet awaits direct experimental confirmation. Without the Higgs mechanism, and its accompanying Higgs boson, the fermions and bosons of the Standard Model have no mass. The Higgs boson has not yet been observed, but the search for the Higgs is ongoing at CDF and D0 and will be a major goal at the Large Hadron Collider when it begins operations in 2007.

There are a number of reasons to be interested in the top quark. The top quark is a fundamental particle in the Standard Model and so characterizing its properties is an important part of studying nature at the most fundamental level. As one of the most recently discovered elementary particles, many of its properties are poorly measured and none are measured as well as we would like. The mass of the top quark is of particular interest. In the Standard Model the mass, in the form of the coupling constant of the top quark to the Higgs boson, is a fundamental parameter and so interesting in its own right. Further, the enormous mass of the top quark, the most massive elementary particle so far observed and far more massive than the other quarks, hints that the top may have some special role among the elementary particles. At minimum, the top has some special connection with the Higgs or with whatever mechanism is responsible for the mass of elementary particles. Finally, the top's large mass means that it has a large impact on any electroweak observ- 
able via radiative corrections. So, precise measurements of the top mass, along with other precision electroweak observables, allow us to constrain the mass of the as yet undiscovered Higgs boson. And, when the Higgs is discovered, these precision electroweak methods will provide an important test of the Standard Model and any successors.

This document reports a measurement of the mass of the top quark using 318 $\mathrm{pb}^{-1}$ of data collected at CDF from March 2002 until August 2004. Events containing top quarks, along with a staggering number of other events, are produced at Fermilab with the complex of accelerators culminating in $1.96 \mathrm{TeV} p \bar{b}$ collisions in the Tevatron. These events are observed with the CDF detector and a tiny fraction of the events, enriched in $t \bar{t}$ events and diminished in backgrounds, is selected. Our measurement is performed with events containing exactly one high energy electron or muon and four "jets" of hadronic energy, the so-called "lepton + jets" channel.

These events are analyzed with a signal and a background probability, each based upon leading-order Standard Model matrix elements. The signal probability asks the question, "What is the probability, given Standard Model expectations that I would observe this particular event?". The signal probability, as a function of top mass, is combined with the background probability event-by-event and all of the events are combined into a joint likelihood. The most probable value of the top mass is then extracted from this likelihood.

However, the leading-order matrix elements are not written in the same language as the events we observe with our detector. In particular, evaluating the matrix elements requires knowledge of quark energies while what we observe in our detectors are several steps removed from the putative quarks. We benefit from many of the same detector calibrations and corrections as other analyses at CDF but we make one significant departure. Instead of using corrections which assign one most probable quark energy to each observed jet, we extract a parton-jet transfer function from simulated events. This transfer function gives the probability distribution of jet energies given the original parton energy.

A number of different top mass measurements have been performed at CDF, with others on the way. They examine different portions of the total available dataset and use different techniques. Our analysis is complementary to these other analyses, but differs in key respects. With the probabilistic matrix element and transfer function based tools we hope to leverage greater statistical information from the same number of events as traditional methods. Further, this measurement is the first in the lepton + jets channel to employ a background probability. 
In the next chapter we describe the theoretical context and the motivation for our measurement in more detail. After this, we provide a description of the Tevatron, the CDF detector, and related technologies. We describe how we select our particular set of 63 events and how they are processed prior to the top mass measurement. Next, we describe our event likelihood and general analysis method in further detail. We proceed to unpack each of its chief constituents: the transfer functions, the signal probability, and the background probability. Having evaluated the performance of the constituents in turn, we then test the full analysis and present expectations for our analysis given the $318 \mathrm{pb}^{-1}$ dataset. We then extract the top mass from data, give an accounting of possible sources of systematic error, perform additional cross-checks, and conclude. 


\section{Chapter 2}

\section{Motivation and Theoretical Context}

There are a large number of reviews and articles of broad scope regarding top physics. I found a number to be especially helpful or of special interest $[7,8,9,10,11,12,13]$. We begin with an overview of the top quark within the Standard Model, where the top is a fundamental particle and its mass, in the form of the Yukawa coupling to the Higgs, is a fundamental parameter of the model. Then, we describe the role of top in precision electroweak physics and its impact on indirect measurements of the mass of the Higgs boson. We then discuss some possibilities for the top beyond the Standard Model and finish by describing the place of our analysis in the context of other top mass measurements at CDF and D0. We will reference some experimental results from CDF and D0 directly, but the latest published and unpublished experimental top physics results are always available from their web pages [21].

\subsection{Top Quark Overview}

In the Standard Model the top quark is the counterpart of the bottom quark in the third generation of quarks. So, it is the weak isospin partner of the bottom quark and so has intrinsic spin $1 / 2$, electric charge $+2 / 3$ and is a color triplet under the strong interaction. To fully characterize top quarks we would like to measure each of these quantum numbers directly and also experimentally determine its mass, lifetime, the Yukawa coupling to the Higgs boson, and the $V_{t b}, V_{t s}$, and $V_{t d}$ parameters of the Cabibbo Kobayashi Maskawa 
(CKM) matrix. Since the top quark was first observed just more than ten years ago and is still only available in modest-size samples most of these parameters have not been measured directly.

In order to discover the top quark, $t \bar{t}$ pair production and subsequent decay have both been observed. The $t \bar{t}$ production cross section has been measured with considerable precision. It is sensitive to the spin of the top and to its coupling with the strong interaction, and observations are consistent with Standard Model expectations. The mass of the top quark is of special interest because it is enormously large. At its current world average mass of $172.7 \pm 2.9 \mathrm{GeV} / c^{2}$ the is more than 30 times as massive as the next heaviest quark, the $b$, and more than four orders of magnitude more massive than the $u$ or $d$ quarks. Yet, as we will discuss, even more precise measurements of the mass are underway and desirable. Studies of top decays have allowed us to conclude directly that $V_{t b}$ is much larger than $V_{t s}$ or $V_{t d}$ but there is much yet to be learned. Comparisons of the kinematics of observed $t \bar{t}$ events with Standard Model expectations have so far revealed no significant deviations, and such general comparisons will become more powerful as more top events become available. With the millions of top quarks expected to be produced each year at the Large Hadron Collider more detailed measurements will be possible and rare processes involving the top quark will become accessible. And, if the possibility of an $e^{+} e^{-}$collider above the $t \bar{t}$ threshold, such as the International Linear Collider, is realized ultra-precise measurements with top quarks will become possible.

\subsubsection{Top Production}

At hadron colliders top quarks are produced by two mechanisms. The most prevalent is $t \bar{t}$ pair production via the strong interaction but top quarks are also produced individually via the electroweak interaction.

Pair production occurs either via $q \bar{q}$ annihilation or by $g g$ fusion, as seen in the leading-order Feynman diagrams of Figure 2.1. That is, the protons and antiprotons accelerated at the Tevatron interact not directly, but via their partonic constituents. At Tevatron energies $t \bar{t}$ production requires collisions between quarks or gluons carrying a significant fraction of the proton energy. During Run 1, with $\sqrt{s}=1.8 \mathrm{TeV}$ (proton-antiproton centerof-mass energy $1.8 \mathrm{TeV}$ ), threshold production was possible if each of the two involved partons carried $20 \%$ of the available energy, which is much more likely for quarks than for 


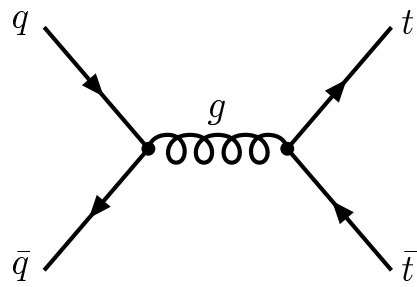

(a)

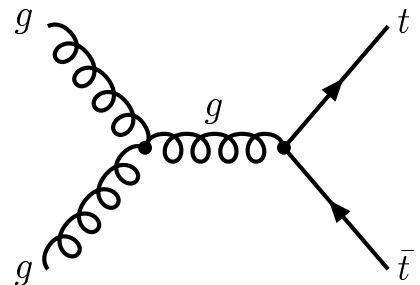

(b)

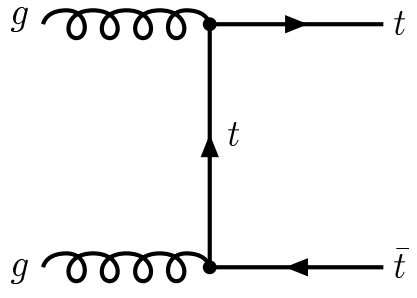

(c)

Figure 2.1: Feynman diagrams for $t \bar{t}$ production via the strong interaction, as at a hadron collider.

gluons. So, during Run $190 \%$ of the cross section was from $q \bar{q}$ and only $10 \%$ from $g g$. Since production is so close to threshold, the modest increase of Run 2 to $\sqrt{s}=1.96 \mathrm{TeV}$ results in a predicted $40 \%$ increase in cross section with $15 \%$ from $\mathrm{gg}$. At the $14 \mathrm{TeV} \mathrm{LHC}$ threshold production will be possible with gluons carrying only $2.5 \%$ of the available. In this regime gluons dominate, so the cross section will be enormous ( $825 \mathrm{pb}$ ) and $90 \%$ from $g g$. In fact, it is this dominance of $g g$ interactions that allows the LHC to collide protons and obviates the need to generate antiprotons.

The $t \bar{t}$ production cross section has been measured at CDF and at D0 in Run 1 and Run 2 in various decay channels and with various techniques. The latest measurements from Run 2 at $\mathrm{CDF}$ are shown in Figure 2.2. State of the art calculations of the $t \bar{t}$ cross section in $1.96 \mathrm{TeV} p \bar{p}$ collisions give $6.70_{-0.88}^{+0.71} \mathrm{pb}$ for $m_{t}=175 \mathrm{GeV} / c^{2}[14,15]$. The most complex complete calculation includes all next-to-leading order (NLO) terms along with a next-to-leading-log (NLL) resummation of softly emitted gluons. Additional NNLO, NNLL, and NNNLL terms have been considered but no such complete calculation exists. The CDF Run 2 average of $7.3 \pm 0.5 \pm 0.6 \pm 0.4 \mathrm{pb}$ is consistent with this expectation.

As has been mentioned, the $t \bar{t}$ cross section depends on the available center-ofmass energy. And, since $t \bar{t}$ pairs have been produced at $\sqrt{s}=1.8 \mathrm{Tev}$ and $1.96 \mathrm{TeV}$ this dependence can be tested, as shown in Figure 2.3. The comparison of Run 1 and Run 2 measurements is consistent with the $40 \%$ increase expected from theory. Unfortunately, the limited statistics available in Run 1 limite the power of this comparison. Of course, the $t \bar{t}$ cross section also depends on $m_{t}$. And, in fact, the CDF measurements of the two quantities are most consistent with Standard Model expectations when this dependence is taken into account as in Figure 2.4.

In the Standard Model top quarks are also produced individually by electroweak 


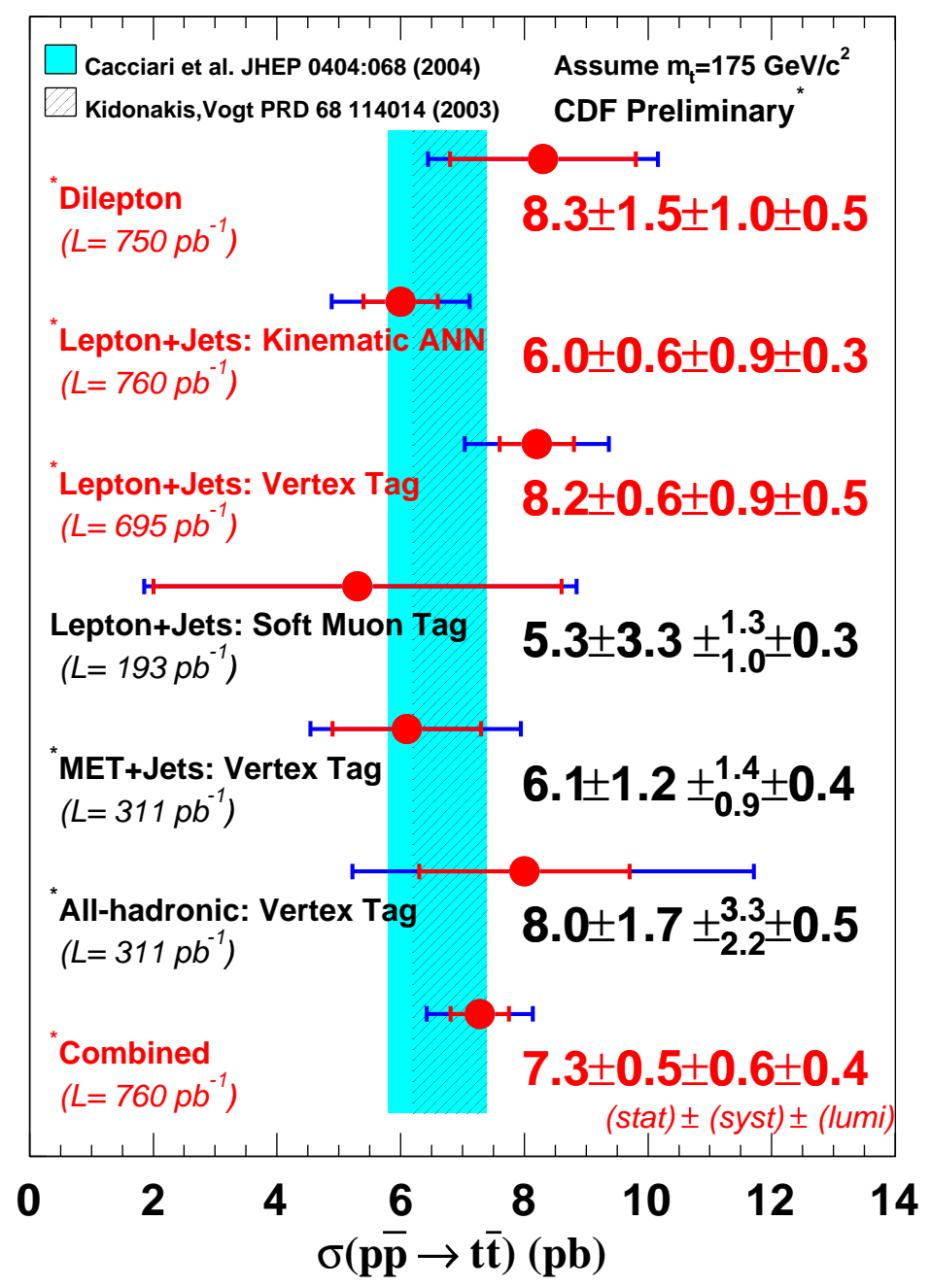




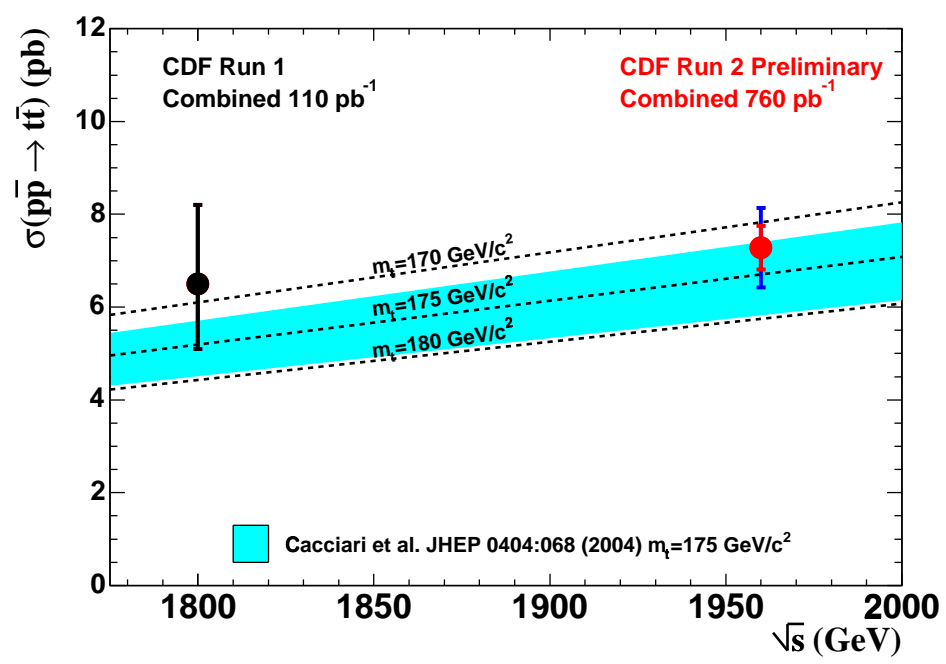

Figure 2.3: The combined Run $1 t \bar{t}$ cross section measurement along with the latest result from Run 2 and the expected $\sqrt{s}$ dependence from Standard Model calculations [14]. The measurements are consistent with the $40 \%$ increase expected from theory.

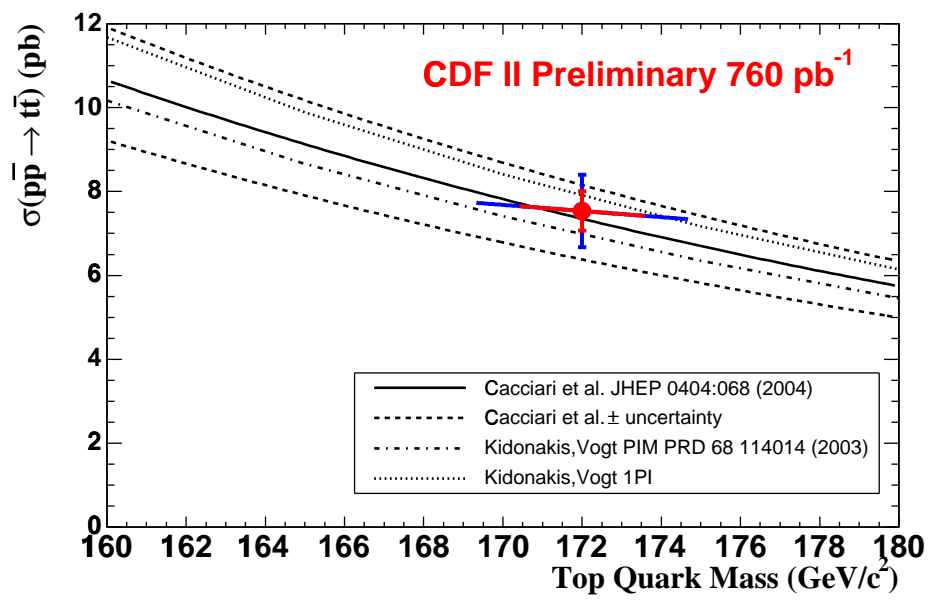

Figure 2.4: The latest CDF combinations (March 2006) of $m_{t}$ and $t \bar{t}$ production cross section are shown along with theoretical expectations for the dependence of the cross section on $m_{t}[14,15]$. The combination of the currently measured values of $7.3 \pm 0.5 \pm 0.6 \pm 0.4 \mathrm{pb}$ and $172.7 \pm 2.9 \mathrm{GeV} / c^{2}$ are consistent with Standard Model expectations. 

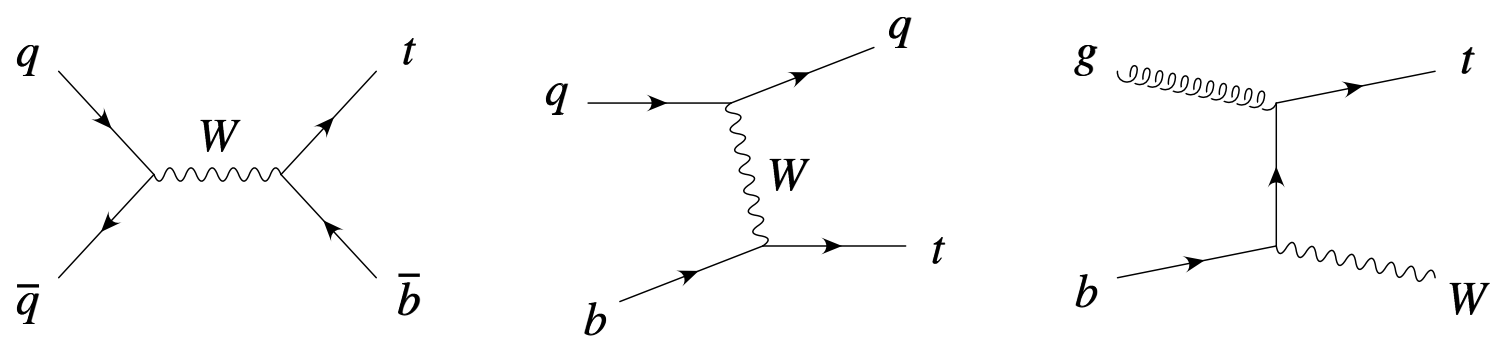

Figure 2.5: Feynman diagrams for single-top production. The first diagram is the $s$-channel subprocess, the second is the $t$-channel subprocess, and the third is one of two diagrams responsible for $W t$ associated production. The $s$ and $t$ channels contribute significantly at the Tevatron, the $t$-channel mostly via $W$-gluon fusion.

interactions. Some of the leading-order Feynman diagrams are shown in Figure 2.5. Because the production is via the electroweak interaction rather than the strong interaction, the cross section is lower than for $t \bar{t}$. At Run 2 of the Tevatron the Standard Model expectations for single-top production are $0.9 \mathrm{pb}$ via the $s$-channel process and $2.0 \mathrm{pb}$ via the $t$-channel. ${ }^{1}$ Compared to $t \bar{t}$ production, fewer events are expected and the backgrounds are considerably more daunting. Searches for single-top production have so far been unsuccessful [54] [55] but limits have not yet reached Standard Model expectations. Single top searches at CDF and D0 will likely reach Standard Model expectations by the end of Run 2, meaning either a discovery or an interesting anomaly.

\subsubsection{Top Decays}

In the Standard Model the branching ratio of top to $b W, B(t \rightarrow b W)>0.998$, that is, a top quark decays to a $W$ boson and a $b$ quark more than $99.8 \%$ of the time. So, $t \bar{t}$ production and decay essentially always occurs as in Figure 2.6. $t \bar{t}$ events may then be characterized according to the decays of the $W$ bosons. Especially important are 30\% of events where one $W$ decays to an electron or a muon and the other decays to quarks, the "lepton + jets" events, and the $5 \%$ of events where each $W$ decays to an electron or a muon, the dilepton events. These two categories of events are the easiest to distinguish at the Tevatron and the most important for top analyses. Esentially all $t \bar{t}$ events also contain $b$ quarks which, along with the $b$ quarks helpfully long lifetime and significant kinematic

\footnotetext{
${ }^{1}$ Both processes involve the exchange of a $W$ boson, one in the $s$ channel, the other in the the $t$ channel.
} 


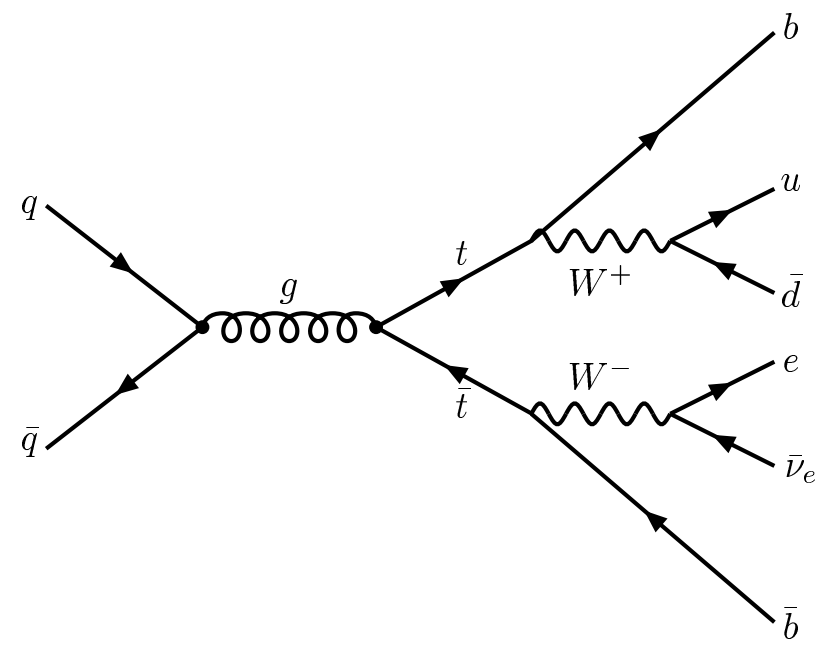

Figure 2.6: Feynman diagram for $t \bar{t}$ production and decay. Specifically, this is production from $q \bar{q}$ and decay into the lepton (electron) + jets channel.

boost frop top decay, makes displaced vertex $b$ tagging a helpful tool for purifying $t \bar{t}$ event samples.

In the Standard Model the top is known to decay so often to $W b$ because of our knowledge of the CKM matrix. The CKM matrix, which connects the weak eigenstates of the quarks to their strong eigenstates, has been explored in considerable detail [17]. While the components involving top have not individually been well measured (almost nothing is known about $V_{t b}$ individually and $V_{t s}$ and $V_{t d}$ are the next least well measured components) the rest of the CKM matrix is very revealing. If there are only three generations of quarks then the unitarity of the CKM matrix indicates $0.9990<\left|V_{t b}\right|<0.9992$. Independent of CKM unitarity $V_{t b}$ is known to be large compared to $V_{t s}$ and $V_{t d}$ as CDF has measured $B(t \rightarrow b W) / B(t \rightarrow q W)=0.94_{-0.24}^{+0.31}$ in Run 1 and that the ratio is $>0.61$ at $95 \%$ confidence level in Run 2 by considering the relative rate of $t \bar{t}$ events with 0,1 , and 2 displaced vertex $b$ tags.

Since the top decays essentially all of the time to $W b$ we can approximate its total decay width as

$$
\Gamma_{t} \approx \Gamma(t \rightarrow W b) \approx \frac{G_{F}}{8 \pi \sqrt{2}} m_{t}^{3}\left|V_{t b}\right|^{2} \approx 1.6 \mathrm{GeV}
$$

at leading order or $1.42 \mathrm{GeV}$ at next-to-leading order. So, the top owes its large decay width to its enormous mass. The decay width is, in turn, responsible for the very short top 
lifetime, $\tau=\hbar / \Gamma \approx 4 \times 10^{-25} \mathrm{~s}$. This short lifetime is actually also responsible, in part, for our ability to measure the top mass so precisely - with a fractional precision much greater than for any other quark. The top decays an order of magnitude more quickly than the characteristic QCD hadronization time and so the top decays as a free quark.

The Standard Model allows for a variety of decays other than $t \rightarrow b W$ but these will be most interesting, and often times only observable, if their branching ratios greatly exceed Standard Model expectations. The decay $t \rightarrow W b g$ is very common and in fact a background for our mass measurement which uses a leading order $t \bar{t}$ matrix element which assumes no gluon radiation. The next most common decays are $t \rightarrow W s, W d$, and $W b \gamma$ the last of which may be useful for directly measuring the top quark's electromagnetic charge. Beyond these are the decays $t \rightarrow W b Z$ and flavor-changing neutral current (FCNC) decays such as $t \rightarrow c g, c \gamma, c Z$. These are beyond the reach of the LHC or even a linear collider in the Standard model, but many scenarios beyond the Standard Model predict large enhancements of FCNC decays.

\subsection{Precision Electroweak Physics}

In the Standard Model, tree-level, leading-order Feynman diagrams like that of $t \bar{t}$ production and decay in Figure 2.6 are only the beginning of the story. To compute a physical process completely we must, in general, include all such Feynman diagrams that have the same incoming and outgoing particle content. The simplest diagrams, such as that of Figure 2.6 are called "tree level" since they contain only straight-line components. The next simplest set of diagrams, necessary for a next-to-leading order calculation, include the one-loop diagrams. For example, the incoming quarks in Figure 2.6, or perhaps the two top quarks, could exchange a gluon.

Similar loops are also possible for the propagators. So, the $W$ boson includes, at next-to-leading-order, the effects of all other particles that interact with it. This includes fermion loops such as the top loop of Figure 2.7. The same is true for the $Z$ boson. So, any process involving $W$ or $Z$ bosons, thus any electroweak observable, is also affected by the properties of the top quark. And, because of the top's enormous mass this effect can be significant.

In the Standard Model the Higgs mechanism is responsible for the masses of the $W$ and $Z$ bosons and also the masses of the fermions. Without the Higgs mechanism the 

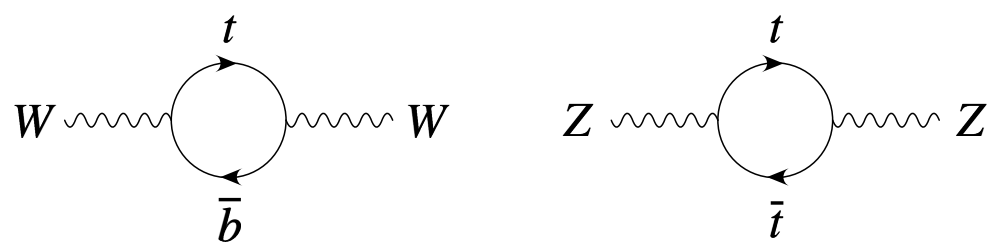

Figure 2.7: One-loop contributions from the top quark to the $W$ and $Z$ masses.

Lagrangian cannot allow masses for the electroweak bosons (or for the fermions) and still be symmetric under electroweak gauge transformations. So, a neutral scalar boson, the Higgs boson, is introduced with a vacuum expectation value. This vacuum expectation value, along with the interaction of the Higgs field with the other fermions and bosons, is responsible for their masses. In the Standard Model the Higgs mechanism is thus responsible for electroweak symmetry breaking (and also flavor symmetry breaking, making the three generations of fermions distinguishable).

At tree level the mass of the $W$ boson is then given by

$$
M_{W}^{2}=\frac{1}{4} g^{2} v^{2}
$$

where $g$ is the $\mathrm{SU}(2)$ coupling constant (the coupling constant of the weak boson field before electroweak symmetry breaking) and $v$ is the vacuum expectation value of the Higgs field. Using the "on-shell" definition of $\sin ^{2} \theta_{W}$

$$
\sin ^{2} \theta_{W} \equiv 1-\frac{M_{W}^{2}}{M_{Z}^{2}}
$$

and relations between three well-measured electroweak quantities $\left(\alpha, G_{F}\right.$, and $\left.M_{Z}\right)$ and the electroweak coupling constants we can rewrite this as

$$
M_{W}^{2}=\frac{\frac{\pi \alpha}{\sqrt{2} G_{F}}}{\sin ^{2} \theta_{W}} .
$$

When we include one-loop corrections this expression is modified:

$$
M_{W}^{2}=\frac{\frac{\pi \alpha}{\sqrt{2} G_{F}}}{\sin ^{2} \theta_{W}(1-\Delta r)},
$$

where $\Delta r$ contains the one-loop corrections. The top quark contribution to the $W$ (and $Z$ ) mass from Figure 2.7 takes the form

$$
(\Delta r)_{\mathrm{top}} \approx-\frac{3 G_{F} m_{t}^{2}}{8 \sqrt{2} \pi^{2}} \frac{1}{\tan ^{2} \theta_{W}},
$$




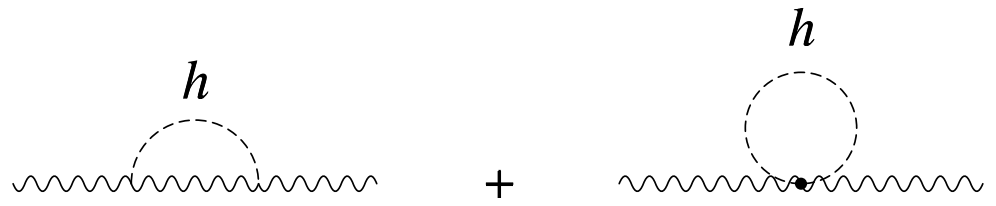

Figure 2.8: One-loop contributions from the Higgs to the $W$ and $Z$ masses.

and so depends quadratically on the top mass.

The Higgs boson similarly contributes to the $W$ and $Z$ masses via the one-loop diagrams of Figure 2.8. The Higgs contribution to $\Delta r$ is given by

$$
(\Delta r)_{\mathrm{Higgs}} \approx \frac{11 G_{F} M_{Z}^{2} \cos ^{2} \theta_{W}}{24 \sqrt{2} \pi^{2}} \ln \frac{m_{h}^{2}}{M_{Z}^{2}},
$$

and depends only logarithmically on the Higgs mass. So, $M_{W}, M_{Z}$, and other electroweak observables are not nearly as dependent on the Higgs mass as they are on the top mass.

Since all electroweak processes involve the $W$ and $Z$ propagators, they are all interrelated and related to the masses of the Higgs boson and the top quark. So, measuring the masses of the $\mathrm{W}$ boson (as has been done at LEP2 and the Tevatron) and the top quark allows us to predict the mass of the Higgs boson, in the context of the Standard Model, as shown in Figure 2.9. The dashed ellipse represents direct measurements of the $W$ mass, from LEP2 and the Tevatron, and the top mass. Diagonal lines of constant Higgs mass are indicated. The lower limit is the $114.4 \mathrm{GeV} / c^{2} 95 \%$ confidence level value from direct searches at LEP2 [16] while theory arguments suggest that the Higgs should not be more massive than $1000 \mathrm{GeV}$ or so. Unfortunately, since the dependence of $M_{W}$ on $m_{h}$ is only logarithmic these two measurements alone do not put a tight constraing on $m_{h}$. They do suggest that the direct measurements of $M_{W}$ and $m_{t}$ prefer a light Higgs and that there is room for reasonable agreeement, within the Standard Model, between the values of $M_{W}$, $m_{t}$, and much of the lower allowed range of $m_{h}$.

Of course, it is also possible to take into account a vast array of other electroweak measurements. Potentially we could include data from deep-inelastic neutrino and electron scattering, atomic parity violation data, and many others. The LEP Electroweak Working Group [18] has combined some such results and a host of data from the $e^{+} e^{-}$colliders at CERN and SLAC in the form of data from SLD, LEP1, LEP2. This data includes results 


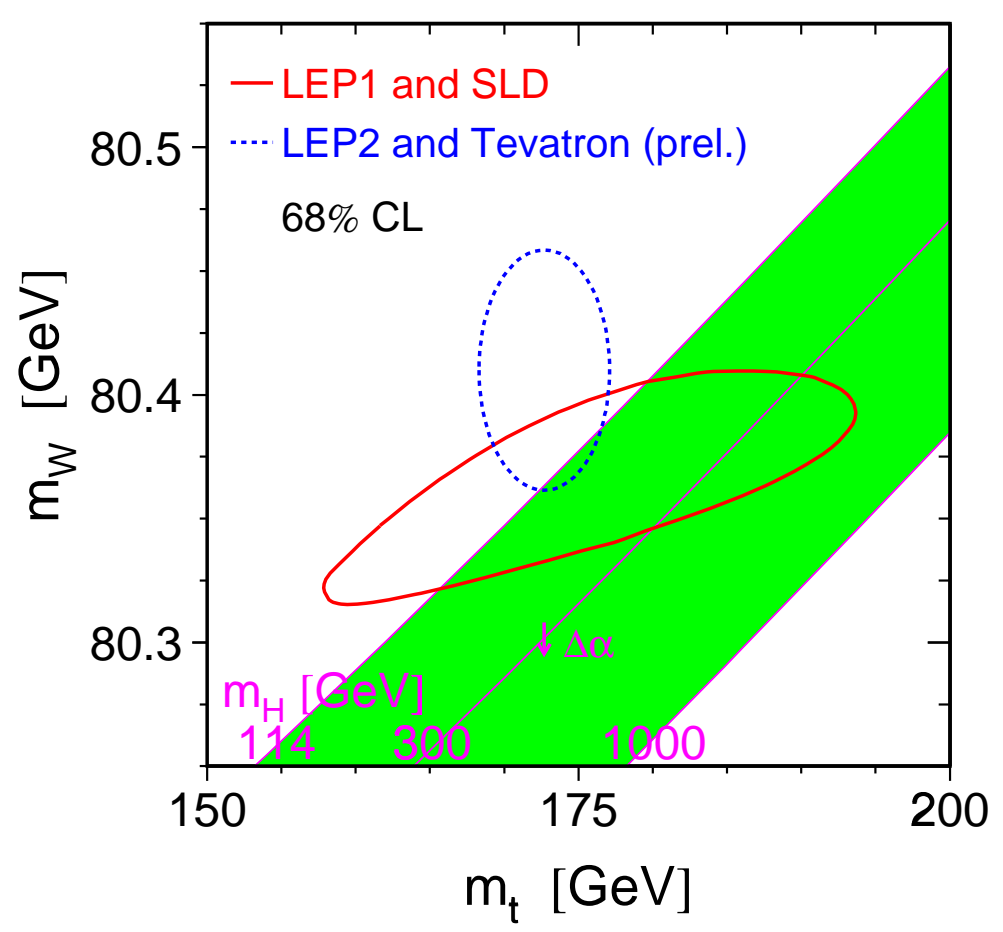

Figure 2.9: The dashed ellipse represents direct measurements of $M_{W}$ (from LEP2 and the Tevatron) and $m_{t}$ (from the Tevatron) while the solid ellipse represents the indirect measurement from precision electroweak data. Lines of constant Higgs mass are shown. Figure from the LEP Electroweak Working Group [18] 
on the masses and widths of the $W$ and $Z$ bosons, forward-backward asymmetries of $b$ and $c$ quarks, and more. All of this precision electroweak data is combined, in the context of the Standard Model, to make predictions (or indirect measurements) of the masses of the $W$ boson and top quark, as given by the solid curved contour in Figure 2.9. The direct and indirect $68 \%$ confidence level contours overlap only slightly, but nevertheless this agreement between direct and indirect measurements is a remarkable test of the consistency of the Standard Model.

It is further possible to combine all relevant precision electroweak data, including direct measurements of $M_{W}$ and $m_{t}$ and leave the Higgs mass as the only unconstrained parameter. These fits are also performed by the LEP Electroweak Working Group, in the Standard Model context. They have the most confidence in the fit including all high $Q^{2}$ data (which neglects some neutrino and atomic physics data). This fit yields a Higgs

mass of $91_{-32}^{+45} \mathrm{GeV} / c^{2}$. This translates into a one-sided $95 \%$ confidence upper limit of 186 $\mathrm{GeV} / c^{2}$ which increases to $219 \mathrm{GeV} / c^{2}$ when the direct search limit from LEP2 is taken into account. The fit is in good agreement with all high $Q^{2}$ data returning, for example, a top mass of $173.3 \pm 2.7 \mathrm{GeV} / c^{2}$, in very good agreement with the inputted world average of $172.7 \pm 2.9 \mathrm{GeV} / c^{2}$. These results suggest that, if the Standard Model is correct, there are Higgs bosons hiding in our data at the Tevatron and that it should be discovered quickly at the LHC. Precise measurements of the top quark mass are a key component of these predictions.

\subsection{Top Quark Beyond the Standard Model}

There is ample reason to suspect that the Standard Model is not the final theory of nature at the fundamental scale. Since any physics beyond the Standard Model should have high energy manifestations a natural place to look is in the most energetic events available, including $t \bar{t}$ events. Any other model of physics will also have to satisfy the constrains and self-consistency check of precision physics, and precision electroweak studies will continue to be a powerful tool for future models.

Probably the most popular set of models for physics beyond the Standard Model is supersymmetry. The simplest case is the minimally supersymmetric version of the Standard Model (MSSM). This extension adds a fermionic (particle with half-integer spin) "superpartner" for every boson (particle with integer spin) in the Standard Model and a bosonic 


\begin{tabular}{|c||c||c|c|c|c|}
\cline { 2 - 6 } \multicolumn{1}{c||}{} & now & Tevatron & LHC & ILC & ILC with GigaZ \\
\hline \hline$\Delta M_{W}[\mathrm{MeV} / c]$ & 34 & 20 & 15 & 10 & 7 \\
\hline$\Delta m_{t}\left[\mathrm{GeV} / c^{2}\right]$ & 2.9 & 2.5 & 1.5 & 0.2 & 0.1 \\
\hline
\end{tabular}

Table 2.1: Current and anticipated experimental uncertainties for $M_{W}$ and $m_{t}$. Each column represents the combined results of all detectors and channels at a given collider, taking into account correlated systematic uncertainties. Table adapted from [19].

superpartner for every fermion. Two Higgs doublets are needed instead of the one doublet of the Standard Model, and a total of five physical Higgs bosons (three neutral and two charged). The MSSM stabilizes the mass of the Higgs boson (eliminating the so-called "gauge hierarchy problem"), aids in the unification of the electroweak and strong interactions, has a dark matter candidate, and is a prerequisite of any string theory.

The implications of precision electroweak physics for the MSSM have been studied in some detail [19]. In Figure 2.10 Standard Model and MSSM predictions of $M_{W}$ as a function of $m_{t}$ are compared to current and anticipated measurements, which are shown in Table 2.1. For the Standard Model prediction the Higgs mass is the only free parameter while for the MSSM the masses of all superpartners are varied in a coordinated manner. The two models show a small overlap - for light Higgs masses in the Standard Model, and for heavy superpartners in the MSSM. The heavy superpartner regime in the MSSM is the socalled decoupling limit where the supersymmetric content has little impact on low energy physics. Current measurements show a mild preference for the MSSM. The anticipated future measurements give a sense for the discriminating power of precision electroweak measurements at, for example, an International Linear Collider.

Another interesting class of theories, those with dynamical electroweak symmetry breaking, do not have fundamental scalar Higgs bosons at all. One possibility is technicolor where the electroweak symmetry is broken by condensates of a new kind of fermions, "technifermions". This symmetry breaking is then communicated to the usual quarks and leptons via a mechanism called extended technicolor which generates the quark and lepton masses. Technicolor theories contain an extended strong interaction which can reduce to two strong interactions at intermediate scales. One can couple to the third generation $(t$ and $b$ quarks) and the other to the other quarks, with the two interactions reducing to the usual strong interaction at low energies. So, it is possible to give the $t$ and $b$ unusually large 


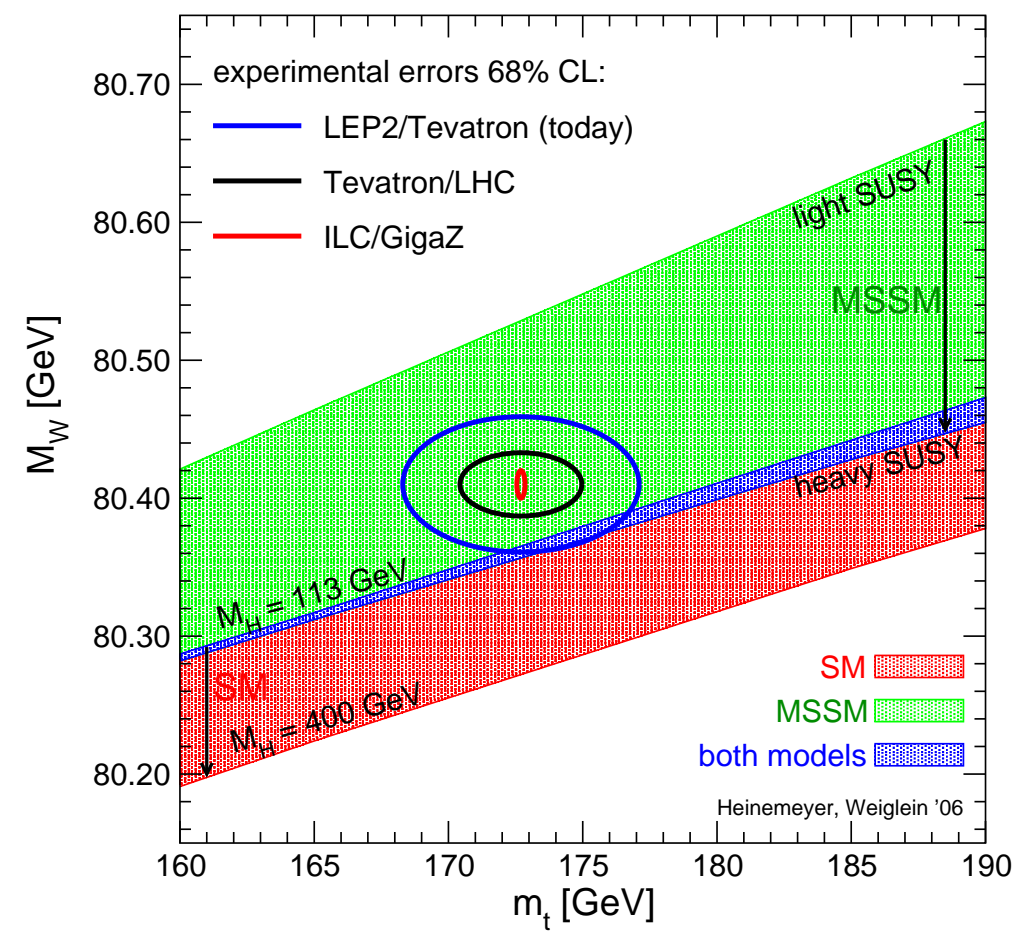

Figure 2.10: The impact of current and future $m_{t}$ and $M_{W}$ measurements in the Standard Model and MSSM. For the Standard Model case the Higgs mass is taken as the only free parameter while the masses of all superpartners is varied. A mild preference is seen for the MSSM over the Standard Model. Figure taken from [19]. Current and future measurements shown in Table 2.1. 
masses. In other versions of such theories, known as topcolor or topcolor-assisted technicolor theories [20], the top plays an even more unique role. In addition to the technifermion condensates the top quark condenses as well. Technicolor causes most of the electroweak symmetry breaking while the top condensate provides most of the top quark mass and the top-bottom mass splitting. This allows large top and bottom quark masses as well as a large top-bottom mass splitting, all while preventing contributions via radiative processes from being unacceptable large. In general any new particles a theory introduces will contribute to processes measurable at low energy via loop diagrams like those of Figures 2.7 and 2.8 and so precision electroweak measurement place tight limits on new theories. One can search for such theories by looking for resonances in the $b \bar{b}$ and $t \bar{t}$ mass spectra that do not exist for lighter quarks. Such searches have been performed at the Tevatron and will continue there and at the Large Hadron Collider.

As one of the highest energy processes currently available for study, top quarks and $t \bar{t}$ events are also a natural place for searches of a general nature for new physics. Top quarks are heavy enough to decay into particles not yet observed, and particles heavier than top could decay into top quarks. For example, in the MSSM a heavy supersymmetric top quarks (stop) could decay into a top quark while if the stop is light the Standard Model top could decay into stop. Heavy intermediate particles from a wide range of theories could decay into $t \bar{t}$ pairs resulting in a possible enhancement in the $t \bar{t}$ cross section and a bump in the $m_{t \bar{t}}$ mass spectrum. So, the $m_{t t b a r}$ spectrum is under study, with no peaks so far observed. And, the kinematics of top quarks events have been, and will continue to be, compared to Standard Model expectations. Any significant deviations would point to new physics - none have so far been observed in Run 2.

\subsection{Other Top Mass Measurements}

The top quark has been of great interest since long before it was discovered in 1995 and so many measurements of its mass have been made. The first such measurements used data from Run 1 of the Tevatron (1992-1996) while more recent measurements, such as the one we describe in this document, are from Run 2. Published Run 1 measurements were combined with then available preliminary Run 2 measurements for an overall result of $172.7 \pm 2.9 \mathrm{GeV} / c^{2}$ with $1.7 \mathrm{GeV} / c^{2}$ of the error being statistical in nature and $2.4 \mathrm{GeV} / c^{2}$ being systematic [22]. The individual contributions to the average are shown in Figure 2.11 


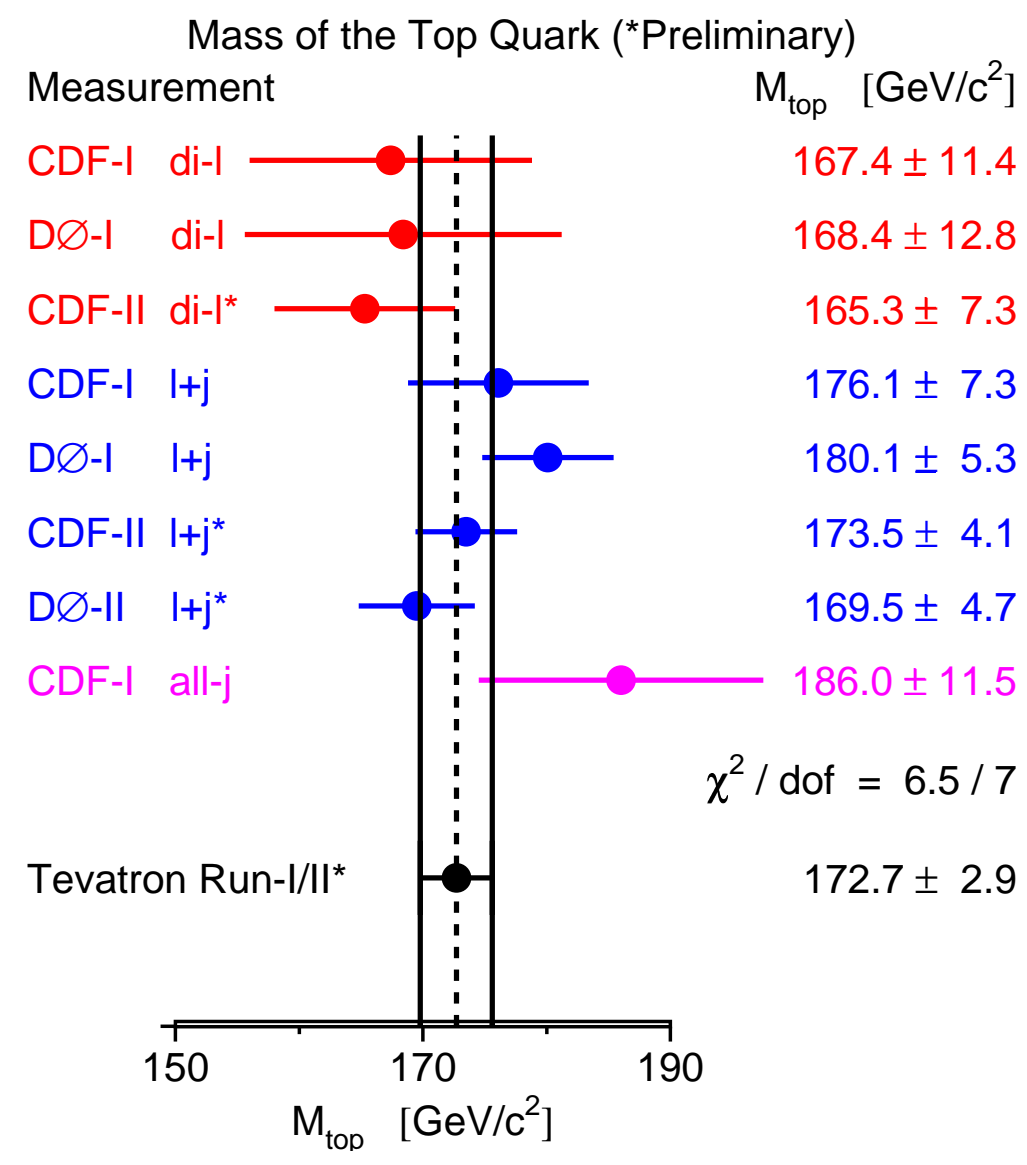

Figure 2.11: Summary of Tevatron $M_{t o p}$ measurements that contribute to the current, preliminary, Tevatron average value of $172.7 \pm 2.9 \mathrm{GeV} / c^{2}$. Plot and average are from the Tevatron Electroweak Working Group [22].

while all Run 2 CDF measurements available at that time are shown in Figure 2.12. CDF and D0 have each made several measurements in the lepton + jets and dilepton channels in Run 1 and Run $2^{2}$ [21] and CDF made a top mass measurement in the all-hadronic channel in Run $1^{3}$. At CDF measurements in the lepton + jets channel have often required a displaced vertex $b$ tag, as is possible also at D0 in Run 2 .

\subsubsection{Template Methods}

Most top mass measurements in Run 1 and many results in Run 2 can be categorized as template methods. The basic idea of a template method is that, for each event

\footnotetext{
${ }^{2}$ The first D0 dilepton top mass results in Run 2 are very recent, from March 2006.

${ }^{3} \mathrm{D} 0$ also released a Run 1 all-hadronic mass measurement in late 2004.
} 


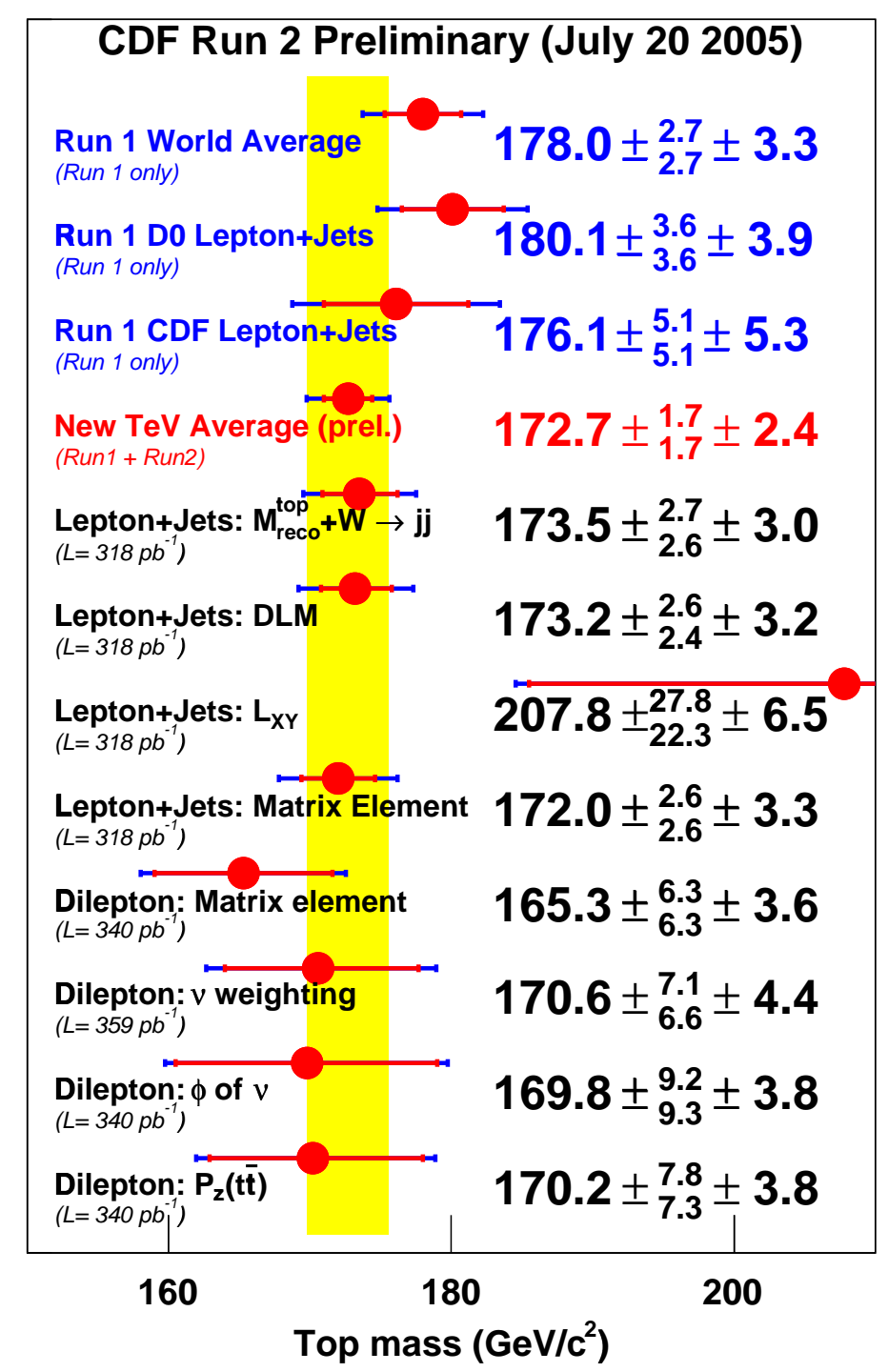

Figure 2.12: Summary of Run $2 m_{t}$ measurements at CDF as of July 2005, along with the best individual results from CDF and D0 in Run 1, the world average from Run 1, and the world average as of July 2005. 
one variable related to the top mass is computed. Using simulated signal and background events templates are constructed for this variable. A series of signal templates are formed for different top masses and various templates are formed for different background processes. The distribution of the selected variable from data is compared with these templates, in what is effectively a shape comparison, and a best-fit top mass is determined. The selected variable could be most anything connected to the top mass, such as $H_{T}$, the scalar sum of transverse energies in the event. But, what is typically chosen is some form of reconstructed top mass. Some set of assumptions about $t \bar{t}$ production and decay are made, and one bestfit kinematic reconstruction of the event is chosen and the reconstructed top mass of that event is calculated from the reconstructed parton and lepton four-vectors. The amount of background in the data sample is generally fit with a constraint indicating the expected amount of background. So, in a traditional template each event is reduced to one number, often a reconstructed top mass. These then enter with equal weight into the final fit, each event having various affinities to each background template or each signal template of a particular mass.

\subsubsection{Matrix Element Methods}

At about the same time as the first Run 2 measurements were made public D0 released a new top mass measurement in the lepton + jets channel, a re-analysis of the Run 1 data set with more sophisticated analytical techniques [23]. These techniques are similar to others previously proposed for use in the dilepton channel $[24,25]$ but the D0 result represents the first time such techniques were used for an actual measurement. The measurement described in this dissertation was patterned in significant part on the D0 reanalysis and so we have already outlined the approach, and will describe it in considerably more detail later in this dissertation.

The D0 re-analysis shares several advantages with a class of analyses which we might call matrix element methods, as opposed to template methods. These advantages relate to the combining of events in the data set, the handling of jet-parton permutations, and the connection between parton energies and jet energies. Together, these enabled significantly more statistical information to be extracted from the same set of events as previous measurements. So, the single D0 measurement was about as precise as the world average of all previous measurements. 
One key way in which matrix element methods depart from traditional template methods is that, instead of representing each event by just one reconstructed top mass, each event is represented by a continuous signal probability, a function of $m_{t}$. So, one event can express preferences for various top masses and the various events in the data set are not combined with equal weights. The D0 re-analysis also included a background probability, and the signal and background probabilities were each calculated from leading-order Standard Model matrix elements. The background probability was especially important for the D0 measurement because in Run 1 D0 had no magnetic field, and thus limited tracking capabilities and no displaced-vertex $b$-tagging. So, the D0 measurement was forced to proceed in the background-heavy pre-tag sample.

The various jet-parton permutations ("Which jet should we assign to the $b$ parton of the hadronic top decay?") are also combined naturally in matrix element methods. The probabilities of the individual permutations are simply added. Template methods typically choose only the one most attractive permutation, thus throwing out the correct permutation a fraction of the time. Matrix-element methods combine all the permutations, allowing them to contribute according to their relative probabilities.

Finally, matrix element methods gain advantage by using jet energy transfer functions. Traditional methods for transforming the jet energies measured in detector calorimeters back to the energies of the original partons ${ }^{4}$ involve a series of physically motivated steps. Some of these steps are common to many analysis while at least one incorporates the specific environment and kinematics of $t \bar{t}$ events. But, traditionally each step is a one-toone function correcting a jet energy to the single most probable corrected energy. Transfer functions, instead, are continuous probability distributions. Given a parton of a certain energy, the transfer function gives the probability that a jet will be observed at various energies. So, instead of allowing only one particular pairing of jet and parton energy, the transfer functions allow all possible pairings, each contributing according to its probability.

\subsubsection{Recent Measurements}

At CDF in Run 2 two lepton + jets measurements of note have so far been published [26]. They were each under development at the same time as the analysis described in this dissertation. The first [27] uses the Dynamical Likelihood Method [25]. It is a ma-

\footnotetext{
${ }^{4}$ In general partons are the constituents of hadrons, so quarks and gluons. From $t \bar{t}$ decays the partons in question are $b, c, u, d$, and $s$ quarks.
} 
trix element method but has several important differences compared to the D0 method, or the method described in this dissertation. First, it generally has somewhat of an a posteriori, as opposed to an a priori approach. Instead of the transfer functions giving the probability distribution of jet energies, given a parton of a particular energy, they give the probability distribution of parton energies, given a particular jet. This seems logical from the perspective of an experiment. After all, we measure jet energies and so it is reasonable to ask, "What is the probability that this jet we have measured came from a 30 (or 35, or 40, etc.) GeV b quark?". But, this necessarily convolutes the particular, mass-dependent, spectrum of parton energies in $t \bar{t}$ events. An additional correction is required to remove this mass-dependence. Our approach, and that of the D0 analysis, is to ask "What is the probability that, given this $40 \mathrm{GeV}$ b quark, we will measure a 25 (or 30, or 35) GeV jet?" We evaluate the matrix element at a particular point in the phase space of the quarks and leptons from $t \bar{t}$ decay and so always know their four-vectors. We then integrate over all possible phase space points. Transfer functions that operate in this direction depend only on the fragmentation properties of the quarks and on detector parameters, and are naturally independent of the mass of the top quark from which the jets originated.

Second, the recent CDF lepton + jets measurement with the Dynamical Likelihood Method uses a signal probability but no background probability. Instead, it studies the bias in its top mass measurement due to simulated backgrounds, and extracts a mapping function to correct for the expected amount of background. Since the measurement requires a $b$ tag there is relatively little expected background and so the loss in precision due to the mapping function is not too great.

The measurement we describe in this dissertation is very similar in concept, but different in a variety of operational details, from the Run 1 D0 re-analysis. So, it incorporates signal and background probabilities and uses transfer functions that map from parton energies to jet energies. But, we require a $b$ tag, use a different background calculation, and take a different approach to the phase space integration of the signal probability. We might expect our analysis to be comparable to the Dynamical Likelihood-based analysis when little background is present, and perhaps to be more adaptable to high-background environments. Certainly the two methods are complementary - taking two related but different approaches to the same goal. A dilepton mass measurement using similar methods to our own has also been performed at CDF, and is awaiting publication [28].

The second recent CDF lepton + jets measurement of note is a template 
method [29], but with significant innovations in the handling of the jet energy scale. While measuring the top mass, the mass of the hadronically decaying $W$ boson is measured in the same event sample. Because the $W$ mass is well known, this allows an in situ measurement of the hadronic jet energy scale. This is the dominant systematic error for top mass measurements in the lepton + jets channel and so an in situ measurement in $t \bar{t}$ events afford a significant reduction in the overall error on the top mass. This template-based measurements stands in a long tradition of template-based top mass measurements at CDF and so much of the analysis is quite mature. This means, for example, that it incorporates separate measurements in the 0,2 , and two different $1 b$ tag sub-samples. Our analysis so far only uses the $\geq 1 b$ tag sample.

Since our analysis was completed in July 2005 a number of other mass measurements have been released at CDF. The current list is shown in Figure 2.13 and the latest measurements are always available electronically [21]. The previously mentioned dilepton measurement has been updated as has the template method with in situ jet energy scale measurement and another novel measurement which measures the top mass directly from the $L_{x y}$ distribution of the $b$ jets. The "Lepton + Jets: Matrix Element" measurement is a descendant of the analysis described in this dissertation and also includes an in situ jet energy scale measurement.

Figure 2.14 shows projections based on the March 2006 results of Figure 2.13. It is meant to give a range of possible future results for the top mass at CDF. The solid line is a relatively conservative extrapolation from current measurements. It assumes that the statistical and the systematic error from the uncertainty on the jet energy scale will scale like the inverse of the square root of the integrated luminosity. Since the number of expected events scales with the integrated luminosity and the jet energy scale is measured from data via the ancillary measurement of the $W$ mass from hadronic jets. The solid line assumes no improvement in the other systematic errors, so that they eventually become the limiting factor in the precision of the top mass measurement. The dashed line assumes that, with additional work, all other systematic errors can be reduced at the same rate.

CDF has already exceeded its goals for top mass precision as outlined in the 1996 Technical Design Report [34], which anticipated a $3 \mathrm{GeV} / c^{2}$ mass measurement. CDF and D0 together, and probably CDF alone, should be able to achieve a $1 \%$ mass measurement before the end of Run 2 and may be able to better event the number anticipated for the LHC in Table 2.1. Of course, we hope the LHC will be able to achieve even more precise 


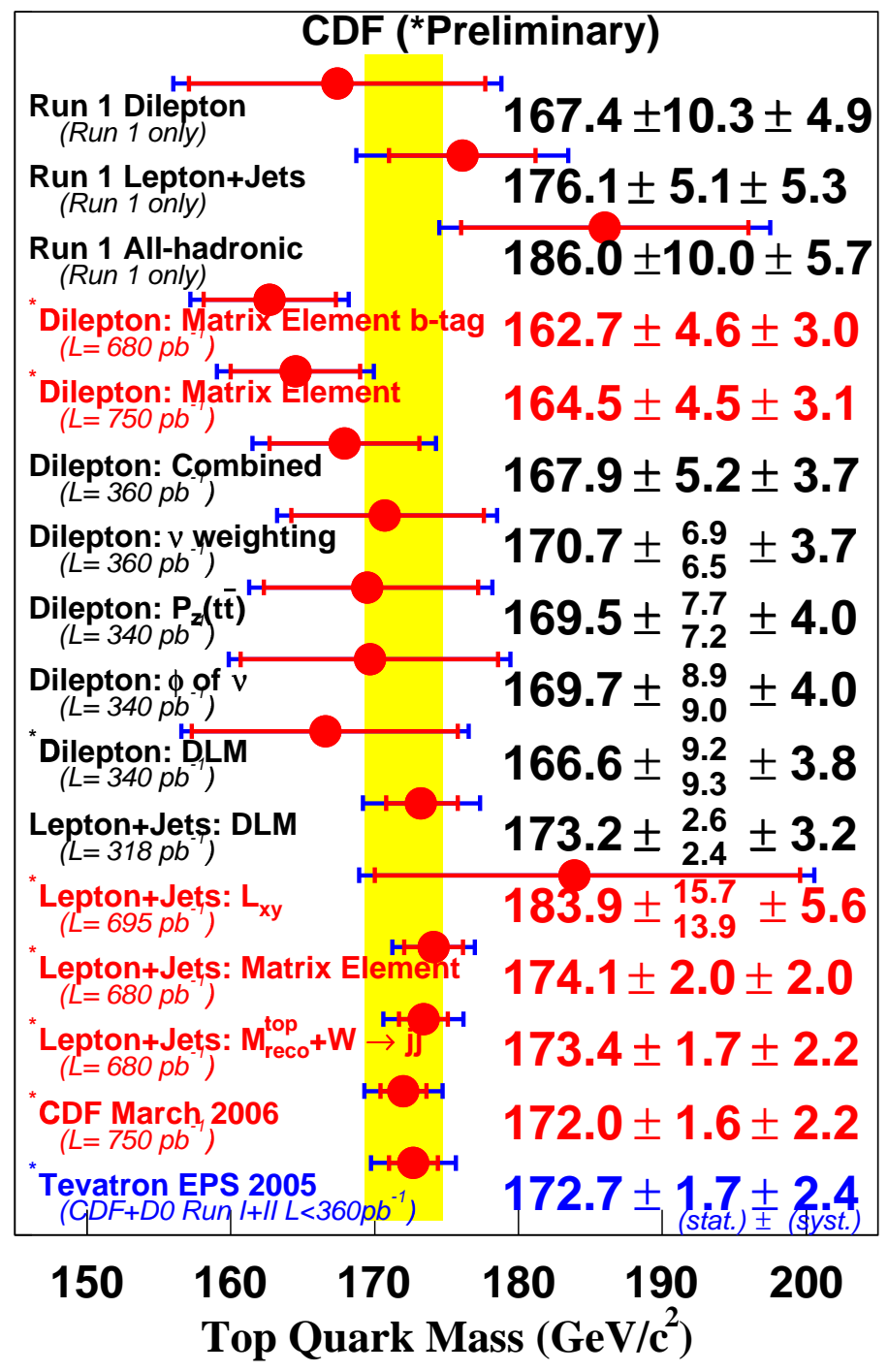

Figure 2.13: Summary of Run $2 m_{t}$ measurements at CDF as of March 2006. Several measurements have been updated with the $750 \mathrm{pb}^{-1}$ data set. The CDF March 2006 is an improvement over the Summer 2005 world average. 


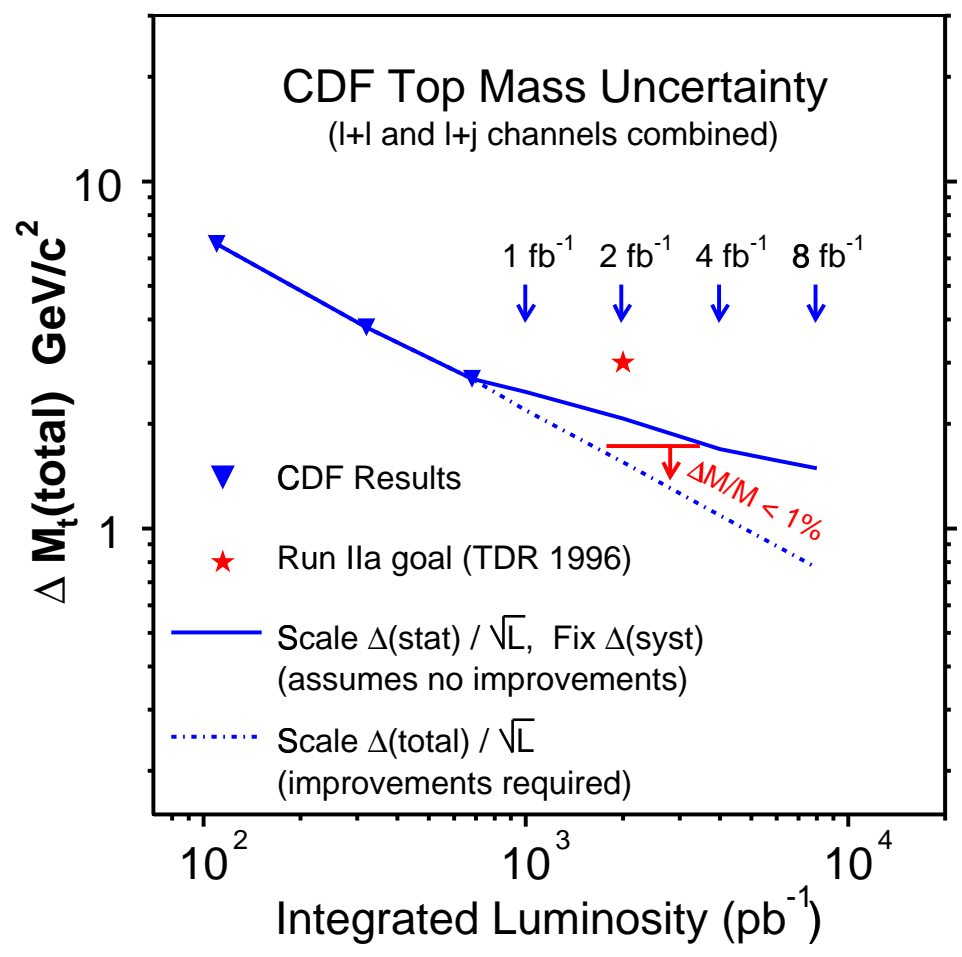

Figure 2.14: CDF projections for Top Mass Uncertainty in Run 2, from March 2006 [30]. The dashed curve scales all uncertainties with the inverse of the square root of the integrated luminosity. The solid curve makes the conservative assumption that the statistical and jet energy scale errors scale like this, but the other systematics remain constant. 
measurements with its much larger $t \bar{t}$ samples. 


\section{Chapter 3}

\section{Experimental Apparatus}

\subsection{Accelerator Complex}

The Tevatron, housed at the Fermi National Accelerator Laboratory (Fermilab), in Batavia, Illinois, is currently the world's highest energy particle accelerator and collider. Until the Large Hadron Collider begins collisions, nominally in 2007, the Tevatron will be the only place on Earth where top quarks can be produced in sufficient numbers to observe them and study their properties. But, the Tevatron is only the last in a series of accelerators responsible for converting hydrogen gas into high energy, high intensity beams of protons and antiprotons $(p \bar{p})$ and bringing them to collision with center-of-mass energy $(\sqrt{s}) 1.96$ $\mathrm{TeV}$. A schematic of the chain of accelerators can be found in Figure 3.1. The information in this section is drawn primarily from the excellent set of "Rookie Books", guides to the accelerator complex for new employees, prepared by the Operations Department of the Fermilab Accelerator Division [31].

\subsubsection{Proton Source, Linac, and Booster}

The accelerator chain begins at one of two possible Cockroft-Walton style electrostatic Preaccelerators. There $\mathrm{H}^{-}$ions (protons with two associated electrons) accelerate from an ion source, kept at $-750 \mathrm{kV}$, to an energy of $750 \mathrm{KeV}$ and are transferred to the Linear Accelerator (Linac). The Linac is approximately $140 \mathrm{~m}$ in overall length, with two distinct sections. The first section, five radio frequency $(\mathrm{RF})$ accelerating cavities with a drift tube design, accelerates the ions from $750 \mathrm{KeV}$ to approximately $166 \mathrm{MeV}$. The second 


\section{FERMILAB'S ACCELERATOR CHAIN}

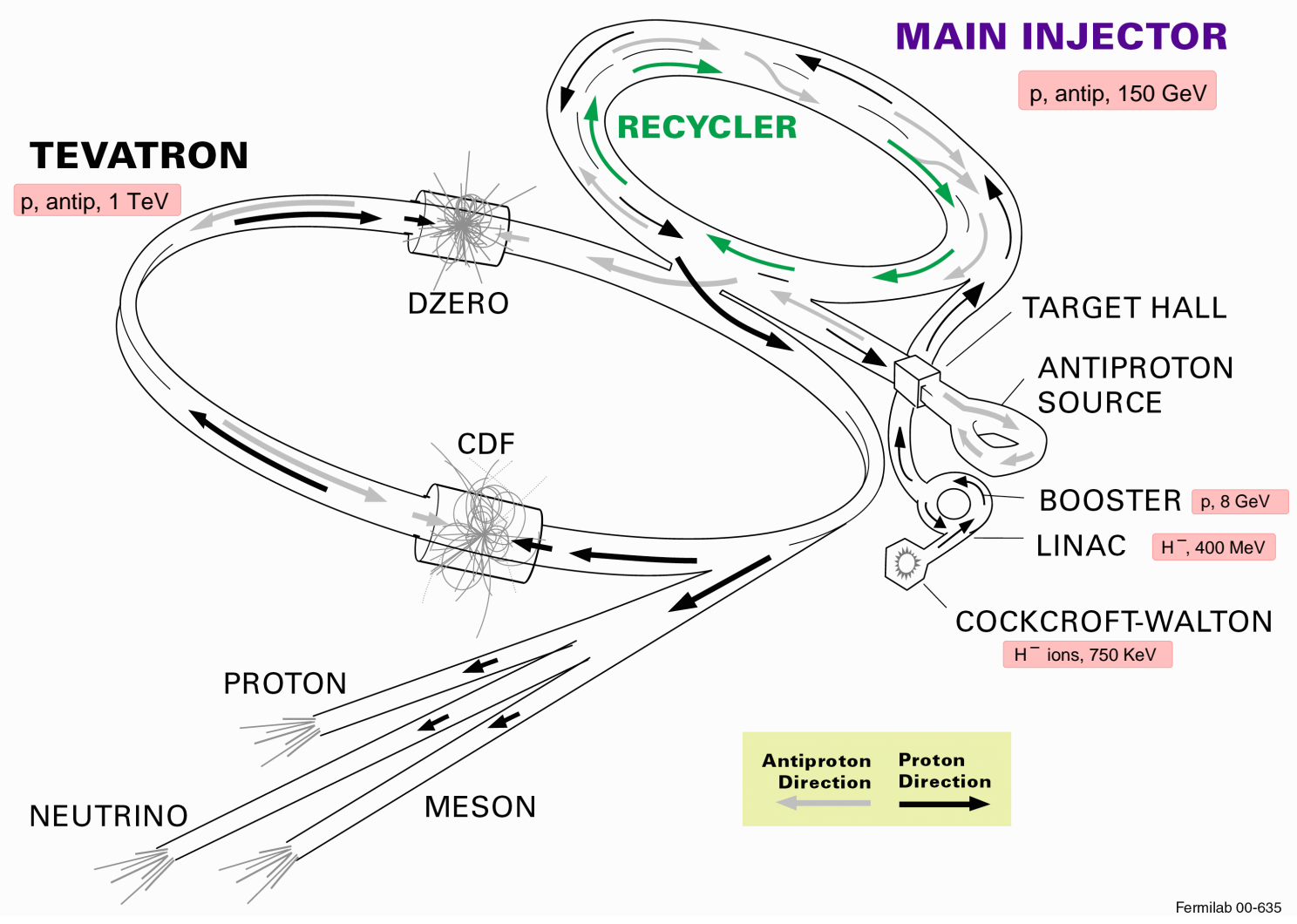

Figure 3.1: Chain of accelerators at Fermilab, culminating in the Tevatron where $980 \mathrm{GeV}$ beams of protons and antiprotons are brought to collision within the CDF and D0 detectors. 
section, 7 RF cavities based on a more modern set of Klystron amplifiers, accelerates the ions the rest of the way to $400 \mathrm{MeV}$. In both sections quadrupole magnets are used to focus the ion beam. After the Linac, the electrons are stripped from the ions, leaving bare protons, which are transferred to the first circular accelerator in the chain, the Booster. This proton synchrotron, with diameter of approximately $150 \mathrm{~m}$, accelerates the proton beam to $8 \mathrm{GeV}$. To maintain circular orbits (e.g. keeping the beam in the vacuum pipe), the dipole magnetic fields in the Booster increase from 0.74 Tesla to 7 Tesla during the acceleration.

\subsubsection{Main Injector}

From the Booster, the proton beam is transferred to the Main Injector, where its destination may be either the Tevatron or the Antiproton Source. In fact, the Main Injectors acts as something of a crossroads for beams of various purposes. The Main Injector is a synchrotron in an approximately elliptical tunnel with circumference of about $3 \mathrm{~km}$. It can accelerate $8 \mathrm{GeV}$ protons from the Booster to either $120 \mathrm{GeV}$ or $150 \mathrm{GeV}$. Protons bound directly for colliding beam operations are accelerated to $150 \mathrm{GeV}$ and transferred to the Tevatron. Other protons are accelerated to $120 \mathrm{GeV}$ for transfer to the antiproton production target and Antiproton Source. $120 \mathrm{GeV}$ protons are also sent to another production target to produce neutrinos for the NuMI (Neutrinos at the Main Injector) project and the MINOS (Main Injector Neutrino Oscillation Search) experiment. The Main Injector also accelerates and transfers antiprotons. It accelerates $8 \mathrm{GeV}$ antiprotons from the Accumulator to $150 \mathrm{GeV}$ for transfer to the Tevatron and colliding beam operations. It also transfers $8 \mathrm{GeV}$ antiprotons from the Accumulator to the Recycler, and potentially (though not in the period of time from which our data comes) receives $150 \mathrm{GeV}$ antiprotons from the Tevatron, decelerating them to $8 \mathrm{GeV}$, and transferring them to the Recycler. Finally, the Main Injector also transfers $8 \mathrm{GeV}$ protons from the Booster to a production target to create neutrinos for MiniBooNE (part of the Booster Neutrino Experiment).

\subsubsection{Antiproton Production and Storage}

The protons for our Tevatron collisions are naturally occurring, in the form of hydrogen gas, but our antiprotons must first be manufactured. So, $120 \mathrm{GeV}$ protons from the Main Injector are steered on to a nickel target. The resulting particle spray contains some antiprotons with a broad momentum distribution, some of which are magnetically 
selected and transferred to the Debuncher. The Debuncher, a rounded triangular-shaped synchrotron with mean radius of 90 meters accepts these antiprotons with a broad momentum distribution and uses a RF manipulation called a bunch rotation to narrow the momentum distribution of the antiprotons at the expense of broadening their time distribution. So, after bunch rotation a given group of antiprotons, a bunch, will have more nearly the same energy and trajectory, at the expense of being more spread out in time (not all antiprotons in the bunch will pass the same point in the ring at the same time). After the bunch rotation, the antiproton beam is transferred to the Accumulator, a second synchrotron and storage ring in the same tunnel as the Accumulator.

Antiproton production is inherently an inefficient process. Only a small fraction of the protons incident on the nickel target produce antiprotons, and only a fraction of these can be usefully retained in the storage rings. Overall, one or two antiprotons are produced for each $10^{5}$ protons incident on the nickel target. The number of antiprotons available for collisions in the Tevatron, and the quality of the beam, is one of the chief limiting factors in the overall Tevatron luminosity and so the number of $t \bar{t}$ pairs that can be produced. So, a variety of techniques are used to maximize the available number, and quality, of antiprotons. First, a variety of cooling techniques are employed to improve the quality (e.g. reduce the momentum spread) of the antiproton beam and increase the number of antiprotons that can be stored for use in collisions. Among these techniques is stochastic cooling [32], a real time feedback mechanism where the beam can be characterized at one location and that information carried across the ring where it can be used to apply corrections to the beam.

Along with cooling mechanisms, to maximize the number of antiprotons available for collision it is also helpful to have more than one antiproton storage ring. So, while all antiprotons from the accumulator are transferred to the Main Injector, some are kept at $8 \mathrm{GeV}$ and transferred to the Recycler, while others are accelerated to $150 \mathrm{GeV}$ and transferred to the Tevatron. The Recycler is another antiproton storage ring located in the same tunnel as the Main Injector. Originally the Recycler was conceived as a means to reuse antiprotons that had already been through colliding beam operations. But, for the data used in this analysis the Recycler is, at most, used as an extension of the Accumulator. The highest luminosity collisions achieved in the Tevatron use a combination of antiprotons from the Accumulator and the Recycler. 


\subsubsection{Tevatron}

The culmination of the chain of accelerators at Fermilab is the Tevatron, a circular synchrotron with $1 \mathrm{~km}$ radius. The Tevatron uses cryogenically cooled, to about $4 \mathrm{~K}$, magnets made of a superconducting niobium-titanium alloy, with the bending magnets having a peak field of more than 4 Tesla. The Tevatron receives $150 \mathrm{GeV}$ protons and antiprotons from the Main Injector, accelerates them to $980 \mathrm{GeV}$, and brings them to collision at two points on the Tevatron ring. These are the locations of the CDF and D0 detectors. There are 36 bunches each of protons and antiprotons co-circulating in the Tevatron and bunch crossing occur every 396 ns.

Run 2 of Tevatron operations began in 2001, with the newly installed Main Injector and the first Tevatron colliding beam operations at $1.96 \mathrm{TeV}$ center of mass energy and 396 ns bunch crossings. The luminosity of the Tevatron early in Run 2 was somewhat lower than expected, but it has been steadily improving especially with the use of the Recycler for stacking additional antiprotons. One batch of protons and antiprotons loaded into the Tevatron is called a store, and may circulate for more than 24 hours. The initial

instantaneous luminosity ${ }^{1}$ for each store, and the cumulative integrated luminosity may be found in Figure 3.2. This analysis uses data from March 2002, when the CDF detector was fully commissioned, to August 2004, representing Tevatron stores between 1120 and 3745 . After selecting data from this period where the detector is operating well, $318 \mathrm{pb}^{-1}$ of data remain for this analysis. Early in Run 2 beam conditions were often too unstable for CDF to safely operate its silicon vertex detectors, essential for identification of b quarks in this analysis, but recent beam conditions have been more favorable.

Parameters from several different Tevatron stores used in our dataset may be found in Table 3.1. They represent three relatively good stores: one from the early period of data taking, one from the late data taking but without the Recycler, and one from late data taking including the use of antiprotons from the Recycler. They represent an increase in instantaneous luminosity of almost a factor of six over two years of operations - a remarkable achievement.

\footnotetext{
${ }^{1}$ The instantaneous luminosity is proportional to the frequency of events of interest, say $t \bar{t}$ production, and is typically measured in $\mathrm{cm}^{-2} \mathrm{~s}^{-1}$. The integrated luminosity $\left(\mathcal{L}=\int L d t\right)$ is a measure of how much total data has been taken and is proportional to the total number of expected events for a process of interest. So, in $318 \mathrm{pb}^{-1}$, if the $t \bar{t}$ production cross section is $6.1 \mathrm{pb}$ we would expect to produce $1939.8 t \bar{t}$ events, on average. Of course, not all of these can be recorded or identified as $t \bar{t}$.
} 

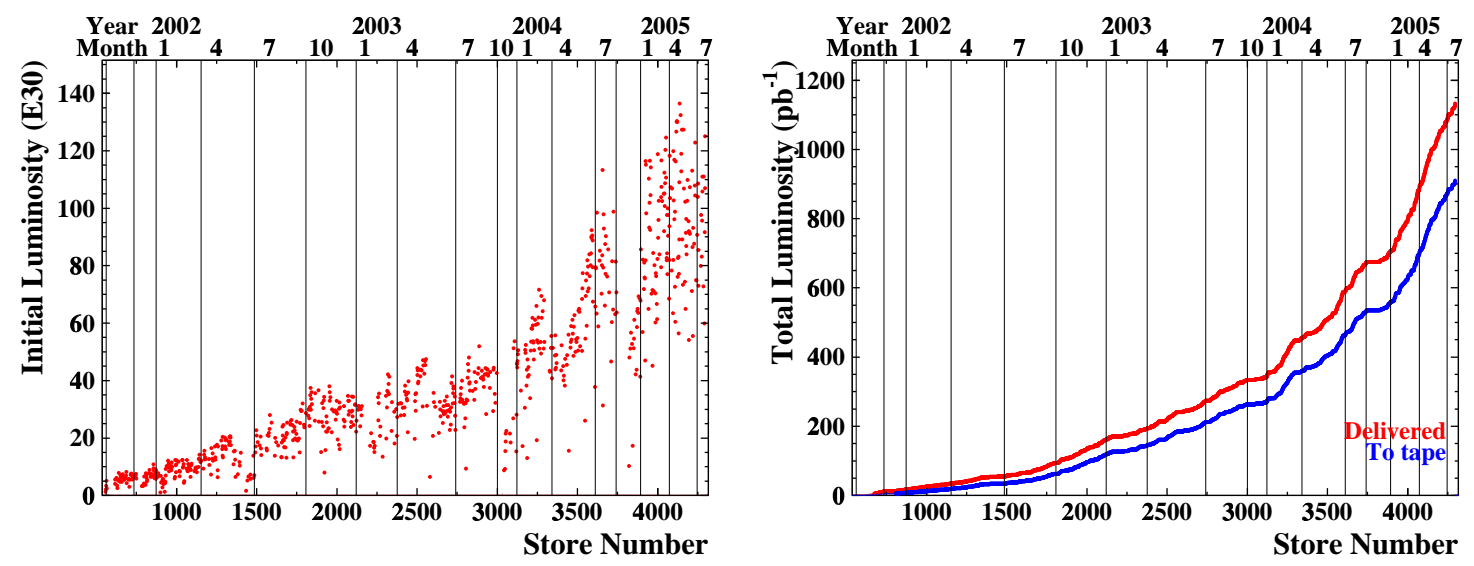

Figure 3.2: Left: Initial instantaneous luminosity for each store. As the accelerator complex has been upgraded, and performance improved, the initial luminosity has greatly increased. Right: Cumulative luminosity vs. store number. The actual amount of luminosity useful for physics analyses is somewhat less than this. So, from stores 1120 to 3745 we use 318 $\mathrm{pb}^{-1}$.

\begin{tabular}{|c|c|c|c|}
\hline Store Number & 1303 & 3717 & 3657 \\
\hline \hline Start Date & May 8, 2002 & August 8, 2004 & July 16, 2004 \\
\hline Length of Store $(\mathrm{hrs})$ & 17.28 & 39.66 & 32.41 \\
\hline Initial Luminosity $\left(10^{30} \mathrm{~cm}^{-2} \mathrm{~s}^{-1}\right)$ & 20.24 & 82.24 & 113.29 \\
\hline Integrated Luminosity $\left(\mathrm{nb}^{-1}\right)$ & 769.33 & 3725.35 & 4456.01 \\
\hline \# of $p$ 's $\left(10^{9}\right)$ & $12,160.89$ & 9096.17 & 8847.94 \\
\hline \# of $\bar{p}$ 's from Accumulator $\left(10^{9}\right)$ & 487.8 & 1027.93 & 1095.31 \\
\hline \# of $\bar{p}$ 's from Recycler $\left(10^{9}\right)$ & 0 & 0 & 460.92 \\
\hline
\end{tabular}

Table 3.1: Selected parameters for several different Tevatron stores. Store 1303 is an example of a good store from early in the dataset used in this analysis, and relatively early in Run 2. Stores 3717 and 3657 represent good stores from the end of the dataset used for this analysis, without and with, respectively, $\bar{p}$ 's from the Recycler. 


\subsection{Collider Detector at Fermilab}

The Collider Detector at Fermilab (CDF Detector) is a general purpose solenoidal detector. To accommodate the varied interests of the approximately 700 physicists who share its data, and to maximize the return on the taxpayers' investment in the Tevatron and $\mathrm{CDF}, \mathrm{CDF}$ attempts to characterize (by measuring 4-momenta and identifying particle type) as many of the particles from as many interesting $p \bar{p}$ collisions as possible. CDF especially emphasizes charged particle tracking and the measurement of central particles, those with large momentum components transverse to the beamline (high $p_{T}$ ). These high $p_{T}$ particles are characteristic of the decays of heavy particles like top quarks, and of very high energy $p \bar{p}$ collisions. This emphasis on tracking and central particles represents a compromise, and a reduced emphasis on calorimetry (the precise measurement of the energies of hadronic particles) and particles close to the beamline (forward particles).

The geometry of the detector is cylindrical, with rough forward-backward and azimuthal symmetry. It is conventional at CDF to use several related sets of coordinates. The $z$ axis points along a tangent to the Tevatron ring, in the direction of proton flight in the accelerator. Transverse quantities such as $E_{T}$ and $p_{T}$ are magnitudes of projections into the plane perpendicular to the $z$ axis. The coordinates $x$ and $y$ (Cartesian) and $r$ and $\phi$ (cylindrical) are defined in this transverse plane, with the $x$ axis pointing outward from the accelerator ring, and the $y$ axis pointing straight up. The angle $\theta$ is the polar angle measured from the proton direction, and $\eta=-\ln \tan \frac{\theta}{2}$ is the pseudorapidity. Typically, $\eta$ is measured from the center of the detector $(0,0,0)$ and is referred to as "detector $\eta$ " occasionally the actual event interaction point is used as the origin to define "event $\eta$ ". The transverse and longitudinal profile of the interaction region are each approximately Gaussian, the former with RMS width of $30 \mu \mathrm{m}$ and the latter with RMS width of $30 \mathrm{~cm}$.

As with most multi-purpose particle detectors CDF takes a multi-layered approach to measure a wide variety of particle interactions. As shown schematically in Figure 3.3 this begins with a tracking volume where the trajectories of charged particles and, via their curvature in the solenoidal magnetic field, their momenta are measured. Progressing radially outward, from the beamline and interaction point out through the detector, we come next to the electromagnetic and hadronic sampling calorimeters. In the electromagnetic calorimeter electrons and photons shower and their energy deposition is measured. Hadrons, like pions, protons, and neutrons, tend to penetrate further, showering in the hadronic 


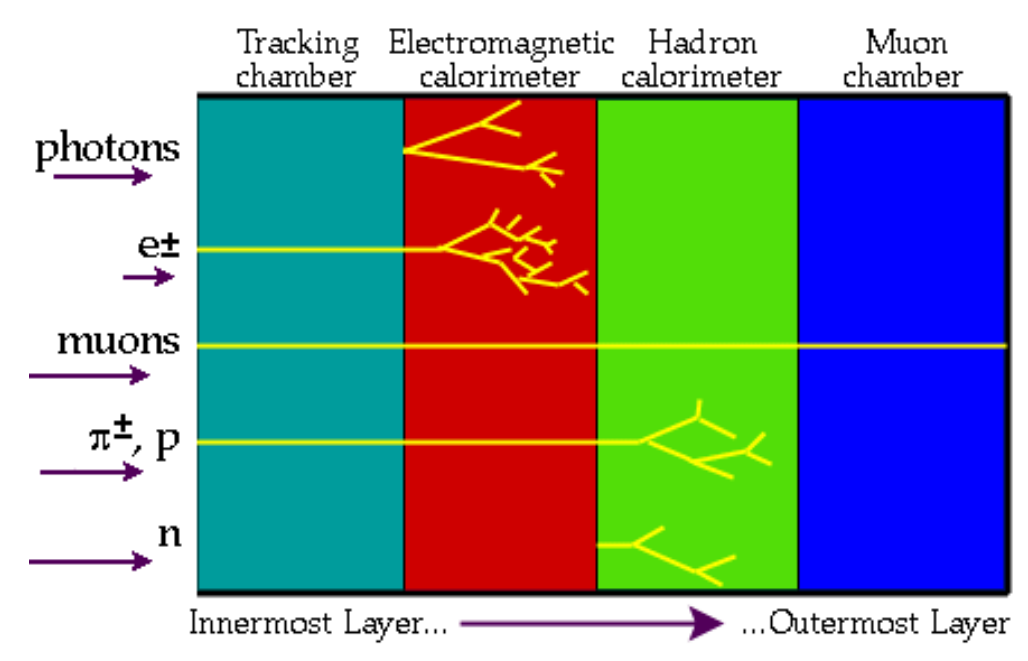

Figure 3.3: Cartoon of particle interactions in detector.

calorimeter. Hadronic jets from quark decays are observed with a combination of the two calorimeter systems. Muons, which are highly penetrating, travel through the calorimeters and are observed in tracking systems outside the rest of the detector. Finally, neutrinos pass through the entire detector without interacting and are inferred from an imbalance of transverse energy in the observed decay products from the $p \bar{p}$ interaction. An overview of the CDF detector can be seen in Figure 3.4 and a clarified view of the tracking system in Figure 3.5.erred from an imbalance of transverse energy in the observed decay products from the $p \bar{p}$ interaction. An overview of the CDF detector can be seen in Figure 3.4 and a clarified view of the tracking system in Figure 3.5.

A single comprehensive reference for the CDF detector in Run 2 does not yet exist. The detector CDF Run 1 detector, from which the current detector has evolved, is concisely described [33]. Many of the upgrades for Run 2 are described in the CDF Technical Design Report [34] and an early Run 2 cross section measurement [35] sometimes serves as a comprehensive reference. We will describe a variety of CDF subsystems in the following sections.

\subsubsection{Silicon Vertex Detector}

The main purpose of a vertex detector is to provide high resolution tracking information close to the interaction point to identify secondary vertices. We would like to detect these vertices, the result of long-lived particles like $B$ hadrons, within even a few $\mathrm{mm}$, in 


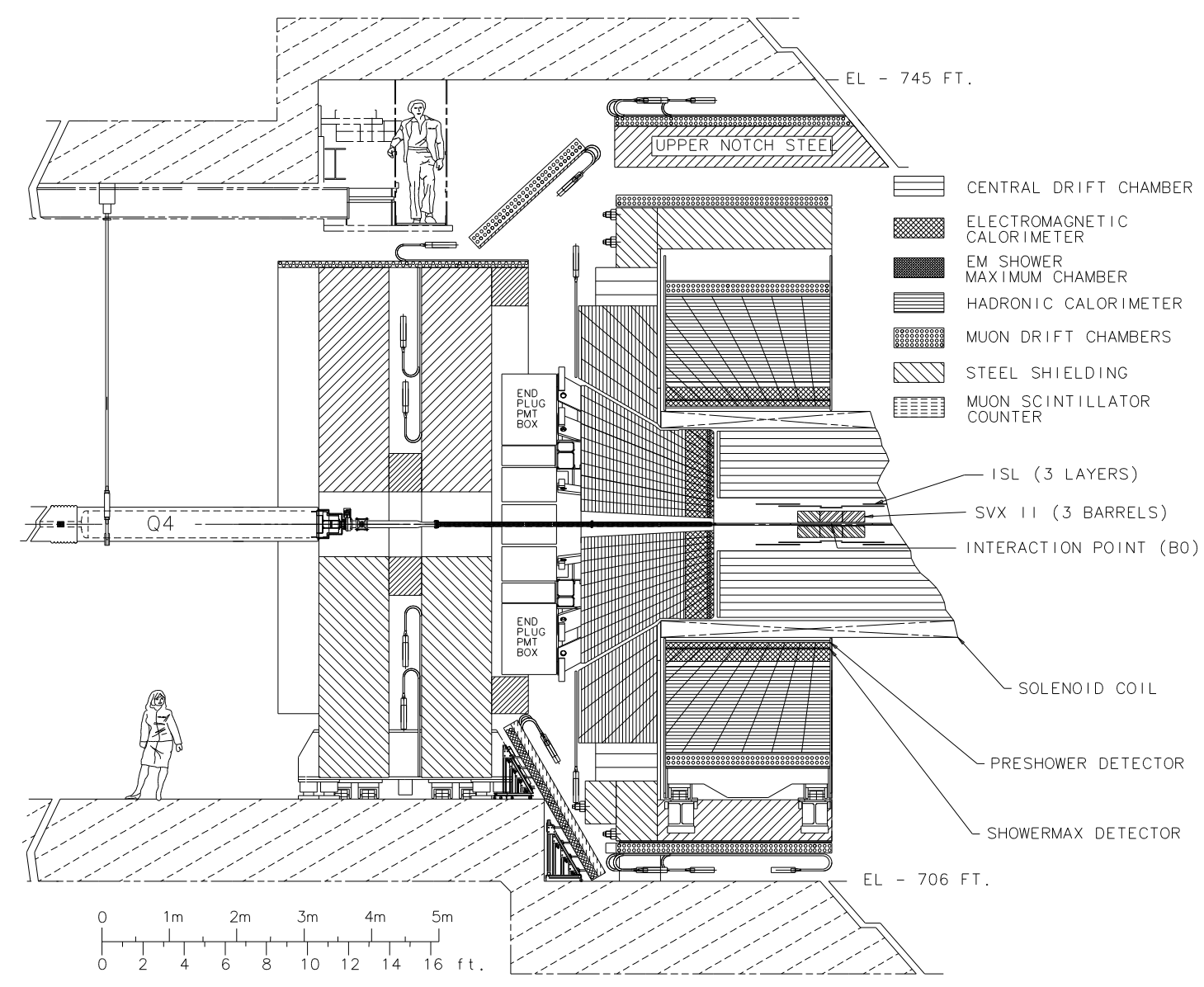

Figure 3.4: An elevation view of the CDF detector. In the central region we see the silicon vertex detector (SVXII) nested inside the open-cell wire drift chamber (COT). These tracking systems are contained in a $1.4 \mathrm{~T}$ solenoidal magnetic field. Then come electromagnetic and sampling calorimeters and muon systems, all of which are also visible in the high $\eta$ region. 


\section{CDF Tracking Volume}

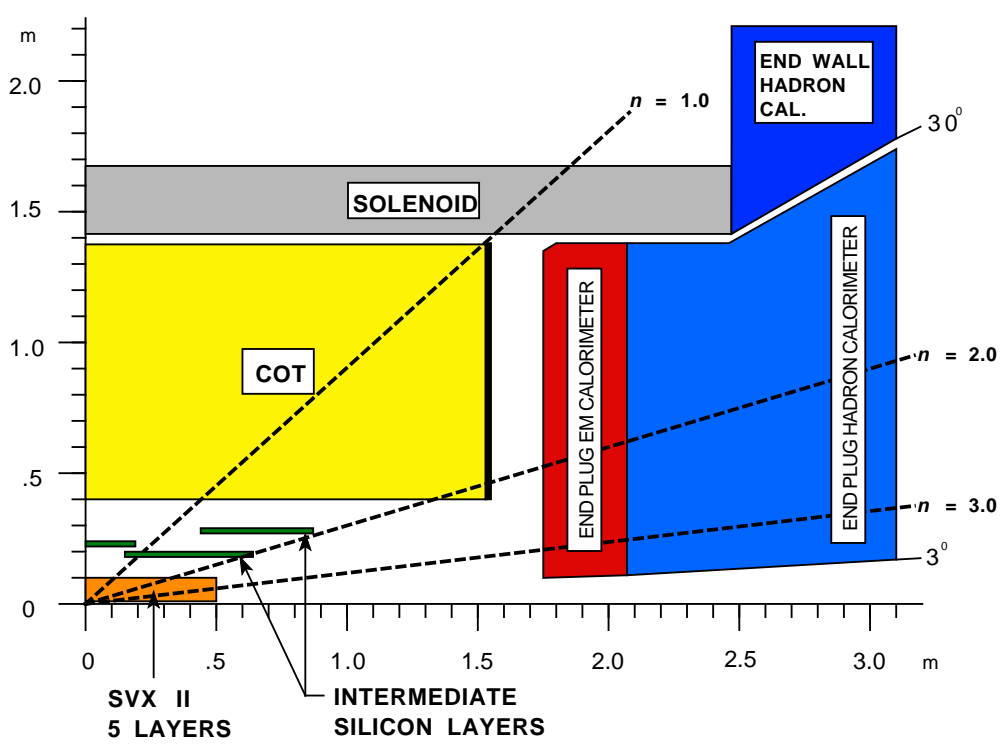

Figure 3.5: The CDF tracking system, here showing one quarter of the overall system.

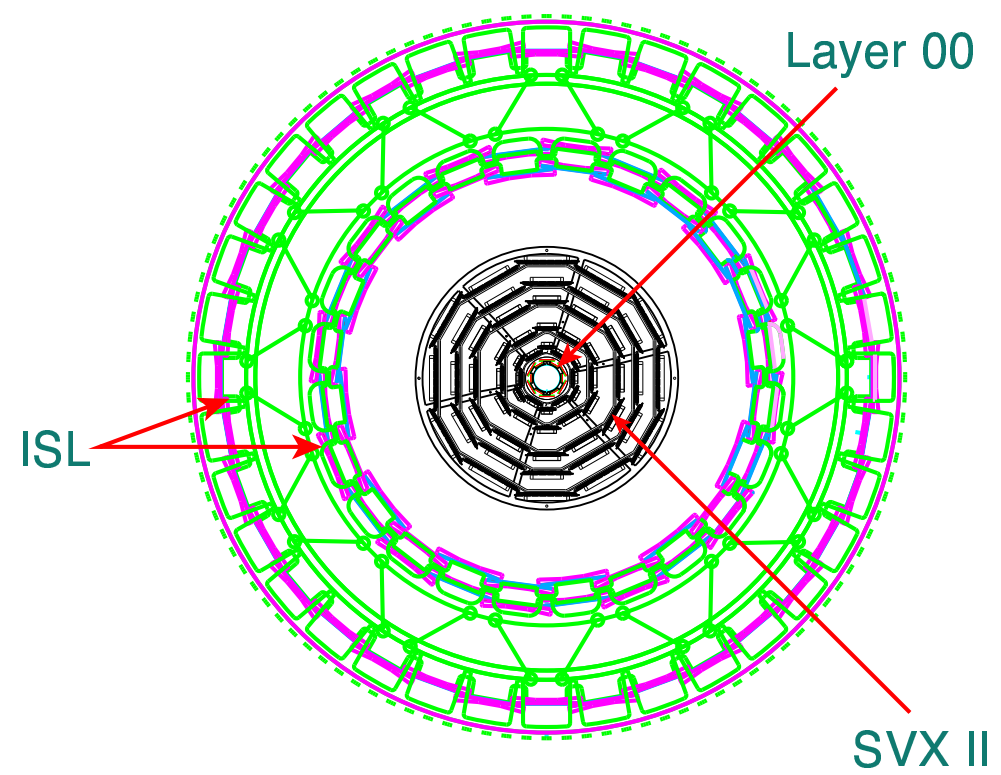
$64 \mathrm{~cm}$

Figure 3.6: An end view of the CDF silicon system. 
the transverse plane, from the interaction point. So, a vertex detector must be close to the beamline, high resolution, and, if we wish to cover most of the luminous region, have very many channels. The solution at CDF is a silicon microstrip detector consisting of three co-axial subsystems [36] as seen in Figure 3.6. There are a total of 722,432 channels in eight layers.

The innermost layer is called Layer 00 which is a radiation-hard, single sided detector. Layer 00 is mounted directly on the beam pipe $(r \approx 1.3 \mathrm{~cm})$ so as to be as close as possible to the interaction point. The SVXII is composed of five double-sided layers $(2.55<r<10.6 \mathrm{~cm})$ while the Intermediate Silicon Layer (ISL) is one double-sided layer in the central region $(r \approx 22 \mathrm{~cm})$ and two in the forward region $(r \approx 20$ and $28 \mathrm{~cm})$ as seen in Figure 3.5. The ISL adds position measurements to those provided by the COT and SVX in the central region, and together with the SVX allows tracking in the forward region where the COT coverage drops off.

The double-sided layers provide $\phi$ measurements with axial strips one side and $z$ measurements with stereo strips on the other. The stereo sides contain strips oriented at either $90^{\circ}$ or $1.2^{\circ}$ from the $z$ axis and allow for the possibility of track reconstruction in three dimensions. Typical strip pitch is $55-65 \mu \mathrm{m}$ for axial strips, $60-75 \mu \mathrm{m}$ for small-angle stereo strips, and $125-145 \mu \mathrm{m}$ for $90^{\circ}$ stereo strips. The impact parameter resolution of the combination of SVXII ad ISL is $40 \mu \mathrm{m}$ including a $30 \mu \mathrm{m}$ contribution from the beamline. The $z_{0}$ resolution of the SVXII and ISL is $70 \mu \mathrm{m}$. In order to minimally disturb particle trajectories, all systems in the tracking volume must have as little mass as possible. For a particle emitted at $z=0$ the entire silicon system, represents several percent of a radiation length at normal incidence, and up to $40 \%$ at $\eta=2$ [37]. This is dominated by passive material (support structures, cooling systems, wiring, etc.) rather than by the sensors themselves.

\subsubsection{Central Outer Tracker: COT}

Tracking in the central region, up to about $|\eta|<1$, is provided over a large radial distance by the Central Outer Tracker (COT) [38]. The COT is an open-cell drift chamber using small drift cells and a fast gas ( $50 \%$ argon, $50 \%$ ethane) to limit drift times to less than 100 ns, such that all of the hits can be read out between each Tevatron bunch crossing.

The basic drift cell has a line of 12 sense wires alternating with field shaper wires 

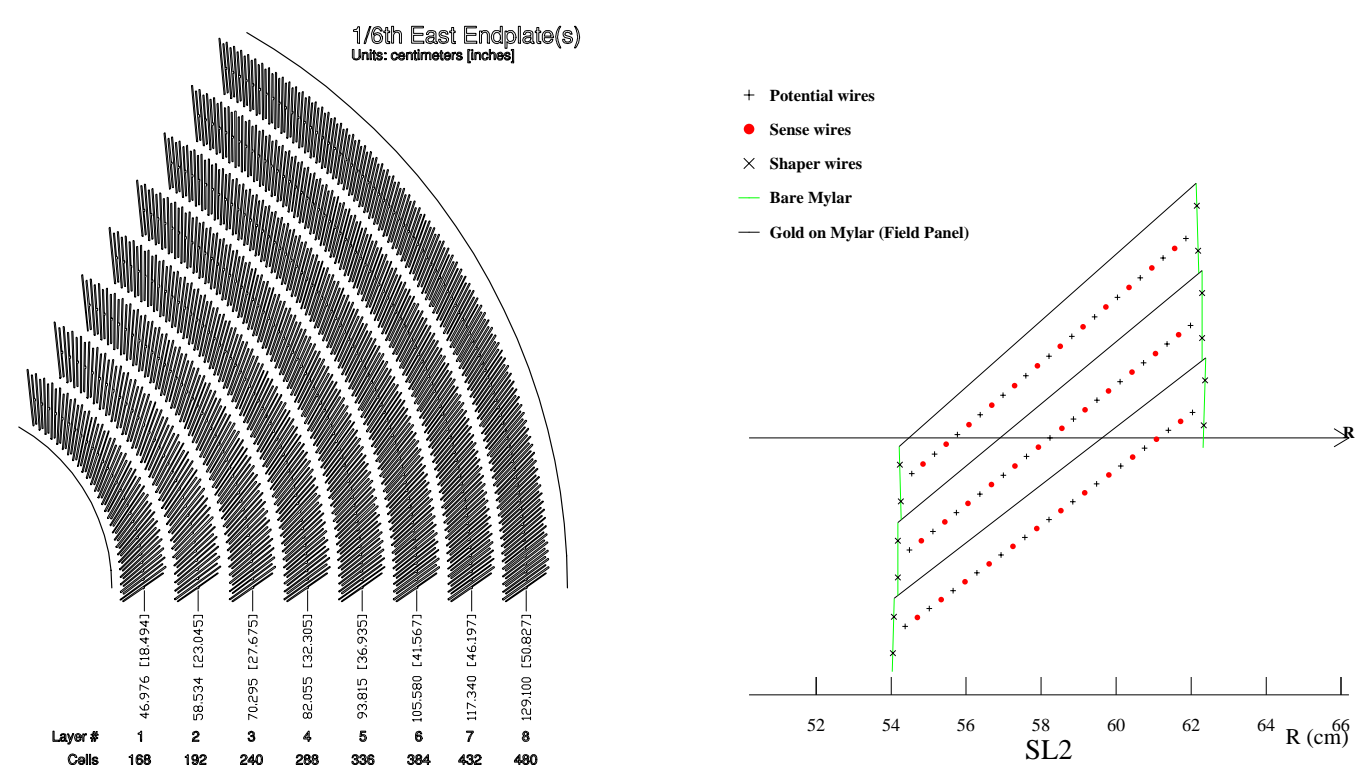

Figure 3.7: Left: 1/6 of the Central Outer Tracker endplate. The machined slots for sense and field planes give a sense of the superlayer geometry.

Right: Cell layout for superlayer 2. The sense ans shape wires and the cathode planes are shown. The COT has a total of 2,520 drift cells and 30,240 readout channels.

every $3.8 \mathrm{~mm}$, all of which is between two gold-on-mylar cathode planes separated by $\approx$ $2 \mathrm{~cm}$. The COT is $3.1 \mathrm{~m}$ in overall length and covers a radial range from 40 to $137 \mathrm{~cm}$. The cells are organized into eight superlayers, alternating between axial and $\pm 2^{\circ}$ stereo superlayers, for a total of 96 measurement layers. The cell and superlayer geometry is displayed in Figure 3.7.

The hit position resolution is approximately $140 \mu \mathrm{m}$ and the momentum resolution $\sigma\left(p_{T}\right) / p_{T}^{2}=0.0015(\mathrm{GeV} / \mathrm{C})^{-1}$. As well as characterizing particle trajectories for momenta measurements the COT also provides $d E / d x$ information for tracks. This information is useful for particle identification helping, for example, to discriminate pions from kaons.

Both the COT and the silicon systems are contained within a superconducting solenoid, $1.5 \mathrm{~m}$ in radius and $4.8 \mathrm{~m}$ in length. A current of $4650 \mathrm{~A}$ goes through the 1164 turns of aluminum=stabilized NbTi to produce a magnetic field of $1.41 \mathrm{~T}$ oriented in the $-z$ direction. The solenoid coil amounts to $85 \%$ of a radiation length at normal incidence, marking the end of the tracking volume and the special concern with minimizing material to avoid pertrubing particle trajectories. By contrast, all of the COT material amounts to $1.69 \%$ of a radiation length, also at normal incidence. 


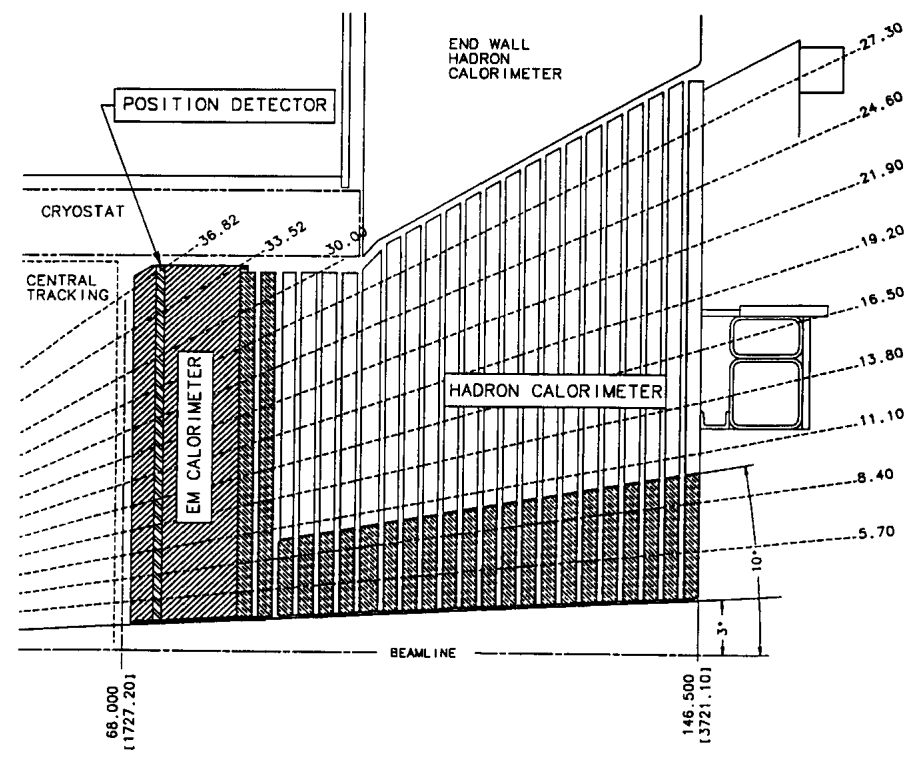

Figure 3.8: View of the forward, or plug, calorimeters (PEM and PHA).

Just outside the COT, in a few centimeters of clearance inside the solenoid, is a Time-of-Flight (TOF) detector [39]. While not used in this analysis, this detector, based on plastic scintillator and fine-mesh photomultipliers is useful for particle identification, using its $\approx 100 \mathrm{ps}$ time resolution to provide two standard deviation separation between $K^{ \pm}$and $\pi^{ \pm}$for momenta $p<1.6 \mathrm{GeV} / \mathrm{c}$.

\subsubsection{Calorimeters}

The purpose of the calorimeters is to measure the energy of particles likely to produce electromagnetic (photons and electrons) or hadronic (hadrons, including neutral hadrons like neutrons) showers as they interact. CDF uses sampling calorimeters, with dense absorbers that initiate and develop showers while absorbing most of the energy in the shower, interleaved with scintillators which convert some of the shower energy, an amount proportional to the total energy, to photons for readout by photomultipliers. The calorimeters at CDF come just outside the solenoid in the central region, and just outside the tracking volume in the forward, or plug, region. The calorimeters are segmented into individual towers which are read out separately. This projective geometry can be seen for the plug calorimeter in Figure 3.8 and also in Figure 3.4. 
The central region is covered by the central electromagnetic [40] (CEM) and hadronic [41] (CHA) calorimeters. The wall hadronic [41] calorimeter (WHA) completes and fills the gap between the plug and central hadronic calorimeters, as labeled in Figures 3.5 and 3.8 and especially visible in Figure 3.4, covering $0.6<|\eta|<1.3$.

The CEM is divided into wedges subtending $15^{\circ}$ in $\phi$ each of which contains ten towers subtending 0.10 units of pseudorapidity, for a total of 480 towers. The absorber layers are each $1 / 8$ inch of aluminum-clad lead, while the scintillator layers are each $5 \mathrm{~mm}$ of polystyrene scintillator, for a total depth of 18 radiation lengths of material. Wavelength shifting sheets collect the photons produced in the scintillator and send them to photomultiplier tubes located behind the calorimeters, as seen in the view of one wedge of the CEM in Figure 3.9. The transverse energy resolution for high-energy electrons and photons is $\frac{\sigma\left(E_{T}\right)}{E_{T}}=\frac{13.5 \%}{\sqrt{E_{T}[\mathrm{GeV}]}} \oplus 2 \%$. Embedded in the CEM at the approximate depth of maximum shower development of the shower $\left(5.9 X_{0}\right)$ are proportional wire chambers (CES). The CES consists of wire proportional chambers with wires and cathode strips providing stereo stereo position information. It can determine the position of an electron shower with a resolution of $2 \mathrm{~mm}$ and is useful in distinguishing electrons from hadronic jets, comparing the shower location with the extrapolation of an electron candidate track. A second set of proportional chambers, the CPR, is located between the CEM and the magnet coil, and is useful for distinguishing photons from neutral pions and hadronic jets.

The CHA and WHA consist of alternating layers of steel absorber $(2.5 \mathrm{~cm}$ per layer for the CHA, $5 \mathrm{~cm}$ for the WHA) and PMMA naphthalene scintillator (1.0 $\mathrm{cm}$ per layer). They are segmented to match the CEM towers, 0.11 units of pseudorapidity per tower and $15^{\circ}$ of azimuth per wedge. There are a total of $384 \mathrm{CHA}$ towers and 288 WHA towers. The total depth of the CHA and WHA are 4.7 and 4.5 interaction lengths, respectively.

The CDF plug calorimeters [42] are similar in concept to the central calorimeters, with electromagnetic and hadronic calorimeters, and shower position and preshower detectors, but the details of the implementation are somewhat different. The plug calorimeters have a more varied tower geometry than the central, but are again projective. There are $48 \phi$ wedges in the lower $\eta$ (less forward) region of the plug, each subtending $7.5^{\circ}$ of $\phi$ and subtending from 0.1 (lowest $\eta$ ) to 0.15 (highest $\eta$ ) units of pseudorapidity. In the most forward region $(|\eta|>2.1)$ there are 24 wedges each subtending $15^{\circ}$ of $\phi$. This makes for a total 480 towers in the plug electromagnetic calorimeter (PEM) and 432 towers in the plug hadronic calorimeter (PHA, with fewer towers because of the existence of the WHA). 


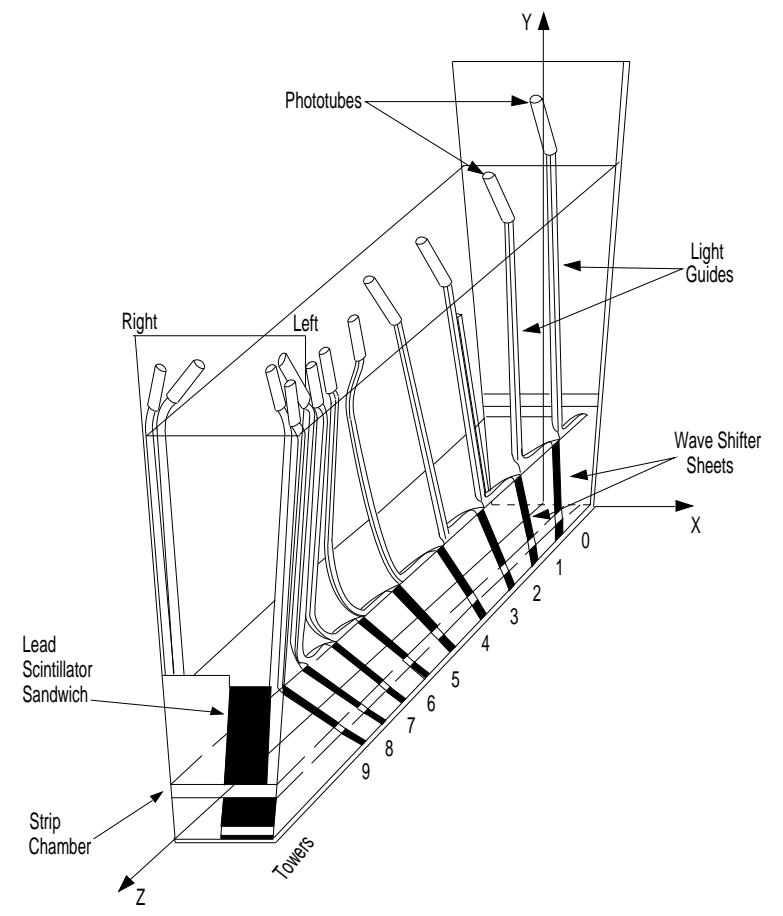

Figure 3.9: One wedge of the central electromagnetic calorimeter (CEM). The strip chambers at shower maximum, $5.9 X_{0}$ into the calorimeter, are indicated.

The PEM alternates 4.55 layers of lead with $4 \mathrm{~mm}$ layers of scintillating tiles, for a total of about 21 radiation lengths at normal incidence. The transverse energy resolution for high-energy electrons and photons is $\frac{\sigma\left(E_{T}\right)}{E_{T}}=\frac{14.4 \%}{\sqrt{E_{T}[G e V]}} \oplus 0.7 \%$. The first layer of scinitillating tiles are read out by multi-anode photomultiplier tubes and act as a preshower detector (PPR) as in the central calorimeter. At the position of maximum shower development (about $6 X_{0}$ ) is located a shower position detector (PES) made of scintillating strips. The plug hadronic calorimeter alternates 2 inch layers of iron absorber with $6 \mathrm{~mm}$ layers of scintalliting tile for a total depth of about 7 interaction lengths.

\subsubsection{Muon Detectors}

The outermost part of the CDF detector are the muon chambers. Muons are detected at CDF by taking advantage of the fact that the muons of interest are minimum ionizing particles, which travel through the calorimeter while losing relatively little energy. So, particles that leave a track in the COT and/or silicon systems, traverse the calorimeter in a matter consistent with minimum ionizing particles, and leave a matching track (stub) 


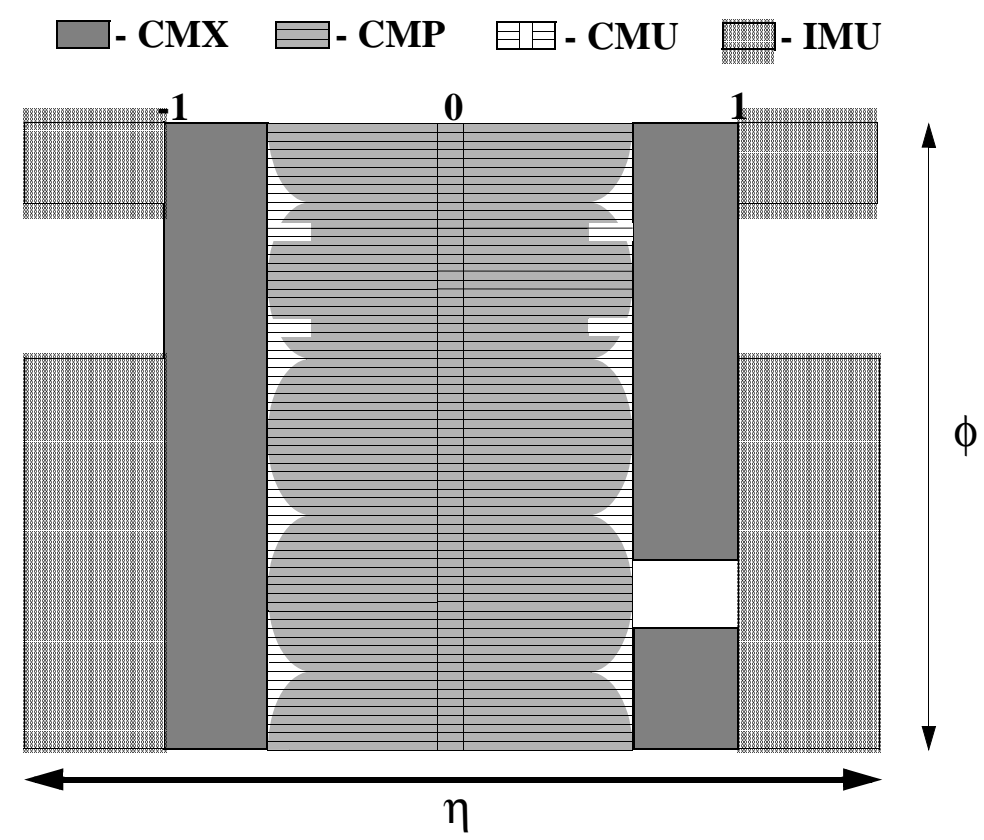

Figure 3.10: $\eta-\phi$ coverage of muon detectors. The IMU is not used in this analysis - we use muons that are detected by the CMX or by both the CMU and CMP.

in the muon detectors, are likely to be muons.

Three muon detectors are used for this analysis. The Central Muon Detector [43] (CMU) and Central Muon Upgrade (CMP) cover $|\eta|<0.6$ while the Central Muon Extension (CMX) covers $0.6|\eta|<1.0$. The CMU is located just outside the central hadronic calorimeter (CHA). It consists of 144 modules of 16 rectangular cells per module, each 6.35 x 2.68 x $226 \mathrm{~cm}$ in size with a $50 \mu \mathrm{m}$ stainless steel wire in the center. The 16 cells in a module are stacked four deep in the radial direction. So, the cells are single-wire drift tubes operated in proportional mode. The CMP is located behind an additional $60 \mathrm{~cm}$ of steel, to reduce the number of hadronic particles that can "punch through" the calorimeters to the muon chambers, and consists of 1076 drift tubes, each, $2.5 \times 15 \times 640 \mathrm{~cm}$ and arranged in a similar four-layer configuration. The CMX contains 2208 total drift tubes, each $2.5 \mathrm{x} 15 \mathrm{x}$ $180 \mathrm{~cm}$, and again in a four-layer configuration. The CMX benefits from no shielding aside from the calorimeter, but because of angle at which muons pass through the calorimeters in reaching the CMX, the total amount of shielding tends to be greater than for the CMU as seen in Figure 3.11.

Because of the size of the individual muon cells, the maximum drift time can be 


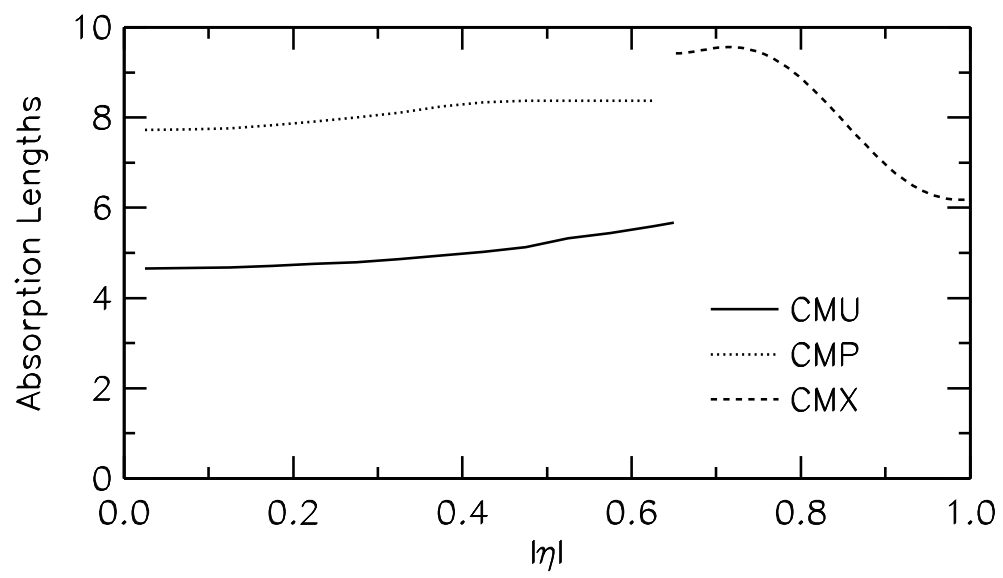

Figure 3.11: The number of absorption, or interaction, lengths through which muons pass before reaching the three muon detectors used for this analysis.

as large as $1.4 \mu \mathrm{s}$, large compared to the $396 \mathrm{~ns}$ bunch crossing time at the Tevatron. The occupancy in the muon detectors is low enough that we can afford to integrate over several events. But, in order to associate the muons with particular events, and for purposes of triggering, a faster system is desirable. So, the muon chambers are accompanied by fast scintillator counters [44].

\subsubsection{Cherenkov Luminosity Counter}

The beam luminosity is determined by using gas Cherenkov counters [45] located in the $3.7<|\eta|<4.7$ region on either end of the detector. These counters measure the average number of inelastic $p \bar{p}$ collisions per bunch crossing. They carry photomultiplier tubes that detect the ultraviolet Cherenkov light emitted by the charged particles traversing the counters.

\subsubsection{Data Acquisition and Trigger System}

With bunch crossing at the Tevatron occurring every $396 \mathrm{~ns}$, a rate of $2.5 \mathrm{MHz}$, we are unable to record all of the $p \bar{p}$ collisions that occur at CDF. Instead, we use a three level trigger system to select the most interesting events, and permanently record events at a maximum rate of about $75 \mathrm{~Hz}$.

The dataflow through the trigger system is shown schematically in Figure 3.12. The trigger system was designed to cope with 132 ns bunch crossings originally envisioned 


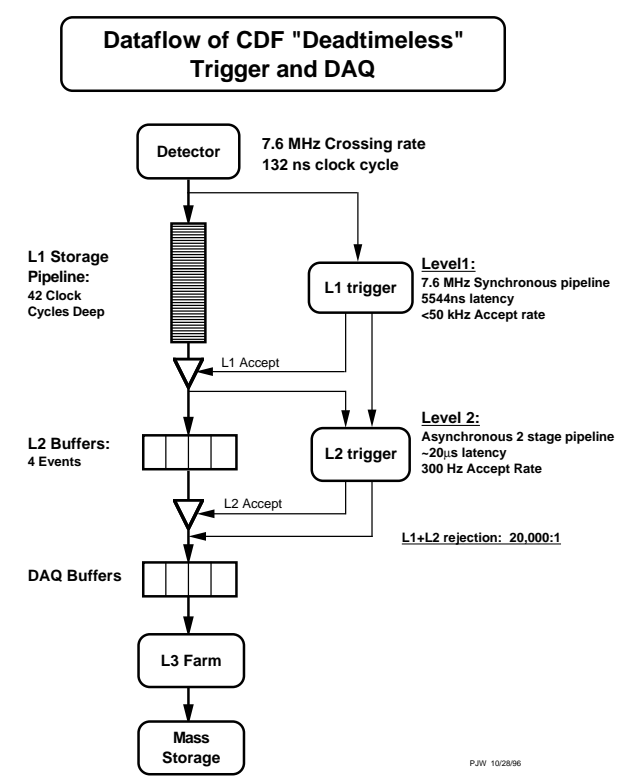

Figure 3.12: Data flow through the CDF trigger system. The crossing rate at the Tevatron is actually only $2.5 \mathrm{MHz}$, but the trigger was designed for the originally envisioned $132 \mathrm{~ns}$, 7.6 $\mathrm{MHz}$ crossings.

for the Tevatron, and so all detector front-end electronics have a synchronous pipeline with on-board buffering where detector signals from every event progress through 42 steps of 132 ns each while waiting for a decision from the Level 1 trigger. Low-level detector information is processed by custom Level 1 trigger electronics where after $5.5 \mu$ s a decision is made whether the event should proceed to Level 2. The Level 1 accept rate is typically 16-20 kHz. When an event passes Level 1 it is stored in one of four available Level 2 buffers while additional reconstruction is performed and Level 2 decision is made after typically $20 \mu \mathrm{s}$. The Level 2 trigger is again implemented in custom electronics. Decisions at Level 2 are asynchronous: events are kept in the buffer for a variable amount of time until a decision is made. The rate of Level 2 accepts is typically 250-300 Hz. If all four Level 2 buffers are occupied when a Level 1 accept is issued the event is lost, and so some dead time is introduced. After being accepted by the Level 2 trigger the entire data event is read out and loaded onto a farm of several hundred commodity PC's. A version of the offline reconstruction, optimized for speed and without final calibrations, is run on each event and events are accepted at rates of typically $60-70 \mathrm{~Hz}$ and permanently stored. Events at level 3 are also available to online monitoring programs run on other workstations, where detector performance is monitored in real time. 


\section{RUN II TRIGGER SYSTEM}

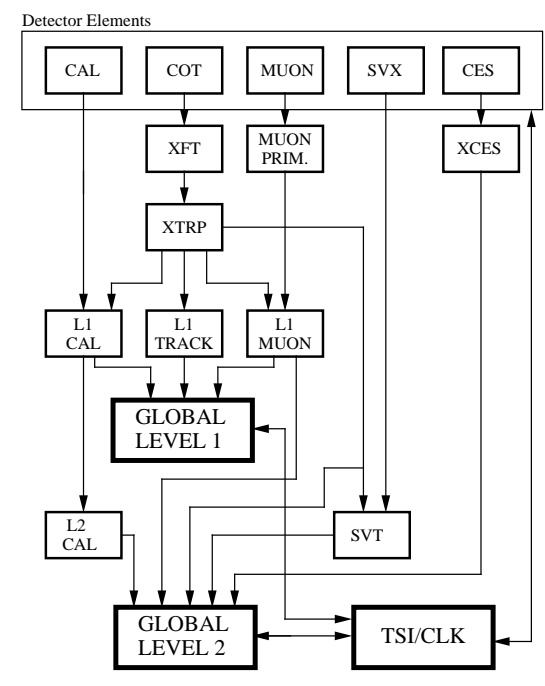

Figure 3.13: Detector information as it is processed and made available to the Level 1 and Level 2 triggers. Particularly notable are the XFT-based COT tracking available at Level 1 and the SVT displaced vertex information available at Level 2.

The logical information available to each level of the trigger is shown in a block diagram as Figure 3.13. Calorimeter, muon, and tracking information from the COT are available at Level 1. A route-based hardware tracking system, the eXtremely Fast Tracker (XFT) distills tracks from patterns of COT hits and these tracks are matched to calorimeter towers and muon tracks in the muon chambers. Level 1 triggering can also be based on calorimeter trigger towers (two physical detector towers) above a certain electromagnetic or total threshold, on the missing transverse energy of the entire calorimeter, or on the total energy deposited in the calorimeter.

At Level 2 information from the silicon vertex detector and the CES shower maximum detector are introduced, and additional processing of calorimeter information is performed. Hadronic jets at CDF generally affect more than one calorimeter tower, and so at level 2 jet clustering is performed to find total jet energies. Shower information from the CES is introduced to help separate jets, electrons, and photons. For the first time at a hadron-collider, the Silicon Vertex Tracker (SVT) provides the ability to trigger on displaced vertices. It reconstructs tracks in the silicon vertex detector, and measures and triggers on their impact parameter. Such triggering is especially helpful for heavy flavor 
physics, the study of $b$ and $c$ quarks. At Level 3 all detector information is available in a format very similar to the full offline event, albeit with primitive calibrations.

\subsubsection{Monte Carlo Event Generation and Detector Simulation}

The ability to accurately simulate the production of physics events and their interaction with the detector is important for almost every analysis, and is essential to this measurement of the top quark mass. We take simulated events based on our best knowledge of underlying physical processes and vary only the mass of the top quark to test our analytical methods. Of course, we treat any uncertainty in our modelling as systematic uncertainties. These events are produced and their interactions with the CDF detector simulated using randomized processes in the form of Monte Carlo (MC) statistical methods.

Our primary event generator for $t \bar{t}$ events is HERWIG [46] while background events and samples for the study of systematic uncertainties are generated using HERWIG, PYTHIA [47], ALPGEN [48], and MadEvent [49]. Parton-level events from ALPGEN are interfaced with HERWIG which provides the fragmentation and hadronization of quarks and gluons from the hard scattering event. Similarly, parton-level events from MadEvent are interfaced with PYTHIA. After fragmentation and hadronization we have, from the generators, a list of typically hundreds of final state particles, and their momenta four-vectors.

These are passed to a detailed description of the CDF detector geometry [50] including the active detector elements as well as much passive material such as read-out electronics, cabling, support structures, and the like. The overall framework for the simulation is GEANT 3 [51] and many interactions with detector matter are handled there. Other interactions are handled with specific parameterized models, such as the GFLASH [52] shower simulation package which is tuned to single particle response and shower shape based on test beam and collision data and used to model particle interactions in the calorimeter. Drift times in the COT and charge deposition in the silicon sensors are also handled by parameterized models outside of GEANT.

The software tools we have just described are of common use among CDF collaborators. We also use modified versions of the ALPGEN and VECBOS [53] to model background events in a way unique to our analysis. That is, we use these codes to calculate a matrix element based probability that an observed event originated through our dominant $\mathrm{W}+4$ parton background mechanism. We describe our implementation of these codes 
later. The ALPGEN-based probability is used in the default background probability four our top mass measurement while the VEBCOS-based probability is used as a cross-check.

The output from the simulation is stored in the same format as real collision data, and is handled by the reconstruction software in substantially the same way as real data. 


\section{Chapter 4}

\section{Selection and Reconstruction of $t \bar{t}$ Events}

\section{1 $t \bar{t}$ Event Characteristics}

Top quarks at the Tevatron are most often produced in $t \bar{t}$ pairs, through quark annihilation $(\approx 85 \%$ during Run 2$)$ and gluon-gluon fusion $(\approx 15 \%$ during Run 2$)$. In the Standard Model they decay almost immediately, with a mean lifetime of $4 \times 10^{-25} \mathrm{~s}$, without enough time even to hadronize. So, their decays are the relatively simple decays of free quarks. For top quarks, given the observed CKM matrix, this means esentially all of the time to a $W$ boson and a $b$ quark. The $W$ 's can then decay either to quarks (e.g. $W^{+} \rightarrow u \bar{d}$ ) or to leptons (e.g. $W^{+} \rightarrow e^{+} \nu_{e}$ ). An example of a Feynman diagram for this process can be found in Figure 4.1.

The $45 \%$ of $t \bar{t}$ events where both $W$ 's decay hadronically are difficult to separate from the multi-jet events abundant at hadron colliders. The two leptons present in the $5 \%$ of events where both $W$ 's decay leptonically represent a clean event signature at a hadron collider, but the two neutrinos complicate reconstruction of the top mass. So, we focus on the $30 \%$ of events where one $W$ decays hadronically and the other decays leptonically. These are the so-called "lepton + jets" events that have historically represented the winning compromise in top mass measurements. We do not specifically target the $20 \%$ of $t \bar{t}$ events with at least one $W$ decaying to a $\tau$ because the $\tau$ decays are difficult to reconstruct. Some $\tau+$ jets events undoubtedly make their way into our event sample and are accounted for in 


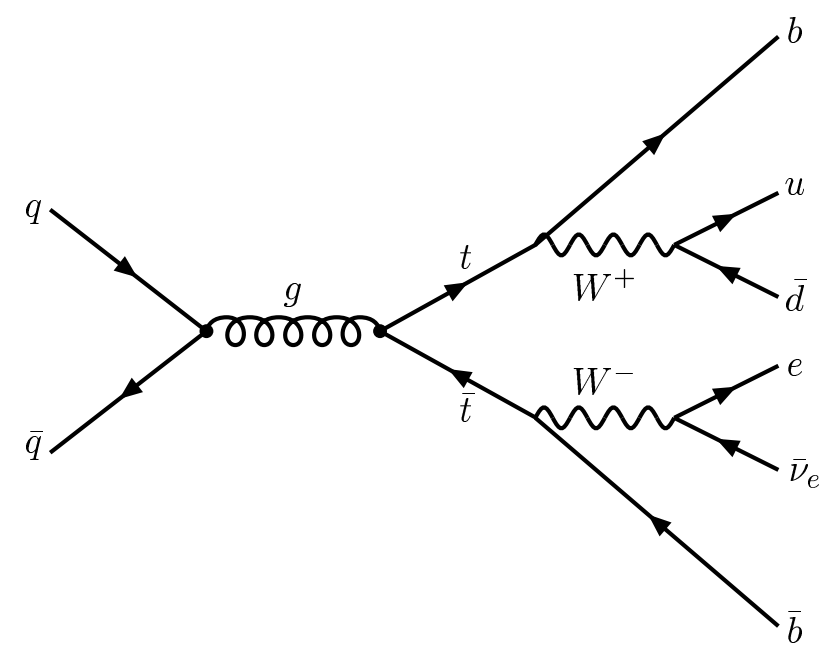

Figure 4.1: Feynman diagram for $t \bar{t}$ production and decay. The gluon-gluon fusion production process is not shown here, and indeed our analysis does not specifically model that process, treating its neglect instead as a systematic uncertainty.

the calibration of our mass measurement. So, here and for the rest of this document when we say "lepton" we mean an electron or a muon, though in its usual sense the term would also include neutrinos and $\tau$ 's.

So, lepton + jets $t \bar{t}$ events contain one electron or muon, two $b$ quarks, two other quarks, and a neutrino. Our task is to select a sample of events enriched in $t \bar{t}$ events, with as little background contamination as possible, and containing events well suited to a precise mass measurement. For example, such events could generally contain additional gluons from initial and final state radiation, but in order to agree with our matrix element's assumption of leading-order $t \bar{t}$ production and decay we veto such events. We will also restrict ourselves to events where all five observable decay particles (neglecting, for now, the neutrino) are measured in well calibrated regions of our detector, as described below. Owing to the large mass of the top quark and $W$ boson each of these six decay products tends to be energetic, typically tens of $\mathrm{GeV}$. This will be the starting point for our event selection which proceeds in two pieces - triggering and offline reconstruction and selection. 


\subsection{Trigger Requirements}

As previously described in Section 3.2.6 the purpose of the trigger at CDF is to sort out the 60 or 75 interesting events that we can write to tape per second from the 2.5 million bunch crossings in that same period. With very few top quarks being produced, less than 20 in the best day of running, the trigger objective for top physics is to record as many $t \bar{t}$ events as possible while not taking up too much of the available trigger bandwidth. For this analysis, where we restrict ourselves to central leptons, triggering is relatively easy. A high transverse energy electron or muon is a reasonably clean signature at a hadron collider and we will get $W$ and $Z$ boson events, interesting in their own right, along in the mix. We use three trigger paths, one for electrons and two for muons.

\subsubsection{Triggering Electrons}

Electrons deposit most of their energy in the electromagnetic calorimeter and leave tracks in the tracking systems. So, to select electron events we begin by requiring, in the Level 1 trigger, a deposit of electromagnetic energy in the calorimeter and a track pointing to it. That is, we require $8 \mathrm{GeV}$ in a central calorimeter trigger tower and that the ratio of energy deposited in the hadronic tower compared to the electromagnetic $\left(E_{\text {had }} / E_{\text {em }}\right.$ be less than $12.5 \%$. We require an XFT track pointing to that tower, with at least 10 (or 11) COT hits in 3 (or 4) superlayers, and with momentum of at least $8.34 \mathrm{GeV} / c$.

At Level 2 we require a calorimeter cluster with $E_{T}$ of at least $16 \mathrm{GeV}$ and with $E_{\text {had }} / E_{e m}<12.5 \%$ for the entire cluster. The cluster is formed by beginning with a seed tower of at least $8 \mathrm{GeV}$ and adding a tower of at least $7.5 \mathrm{GeV}$, adjacent in $\eta$, if available. The XFT track must point to the seed tower of the cluster.

At Level 3 we require an offline central electromagnetic cluster with $E_{T}>18$ $\mathrm{GeV}$ and $E_{\text {had }} / E_{\text {em }}<12.5 \%$ with a matched COT track with $p_{T}>9 \mathrm{GeV} / c$. The lateral sharing of energy between calorimeter towers in the cluster must agree with that of test beam electrons, and the centroid of a CES cluster must agree with the extrapolated track position to within $8 \mathrm{~cm}$ in $z$.

Trigger requirements have evolved somewhat over the time period used in this analysis in order to optimize trigger rates, efficiencies, and fake rates as instantaneous

luminosity has increased. Typical trigger rates for this electron trigger path are $80 \mathrm{~Hz}$ at Level $1,8 \mathrm{~Hz}$ at Level 2, and $1 \mathrm{~Hz}$ at Level 3. The efficiency of this trigger path has been 
found to be $0.9656 \pm 0.0006$ for events that would otherwise pass an offline lepton + jets event selection [56] similar to ours.

\subsubsection{Triggering Muons}

For muons we have two similar trigger paths, one for muons that are detected in both the CMU and the CMP (CMUP) $(|\eta|<0.6)$ and another in the CMX $(0.6<|\eta|<1.0)$. They begin by requiring a muon "stub", a collection of related hits in subsequent layers of the muon chambers. A CMU stub requires corroborating hits in the CMP, and both trigger

paths require a corresponding XFT track $(4.09 \mathrm{GeV} / c$ for the CMUP, $8.34 \mathrm{GeV} / c$ for the CMX). At Level 2 the XFT requirement is tightened, to $8.34 \mathrm{GeV} / c$ in the CMUP and 10.1 $\mathrm{GeV} / c$ in the CMX. At Level 3 the position of the centroid of a CES cluster must match the extrapolated track position in the $r-\phi$ plane to within $10 \mathrm{~cm}$ in the CMU or CMX, and for CMUP muons also within $20 \mathrm{~cm}$ in the CMP.

Typical trigger rates for these muon trigger paths are $90 \mathrm{~Hz}$ at Level $1,9 \mathrm{~Hz}$ at Level 2, and $0.4 \mathrm{~Hz}$ at Level 3 for the CMUP path and $9 \mathrm{~Hz}$ at Level 1, $7 \mathrm{~Hz}$ at Level 2, and $0.25 \mathrm{~Hz}$ at Level 3 for the CMX path. The efficiency of the CMUP trigger path has been found to be $0.887 \pm 0.007(0.954 \pm 0.006$ for the CMX) for events that would otherwise pass an offline lepton + jets event selection [56] similar to ours.

\subsection{Offline Event Reconstruction}

The trigger requirements, described above, determine which events are permanently stored. But, so far, the events we have described are distinguished only by a high $E_{T}$ electron or high $p_{T}$ muon. So, it is a sample enriched in $W$ and $Z$ bosons as well as with top quarks. Offline, we require four high $E_{T}$ jets, the product of four quarks after hadronization, and apply corrections to their measured energies. We require large missing $E_{T}$ as a sign of the neutrino from $W$ decay, impose additional quality and fiduciality requirements on electrons and muons, and reject events with more than one high energy lepton or with other event topologies likely to come from background contamination. We also require at least one jet to have a secondary vertex as evidence of a $b$ quark. 


\subsubsection{Offline Electron Reconstruction and Selection}

Offline electrons begin with an electromagnetic (EM) cluster in the CEM with $E_{T}>20 \mathrm{GeV}$. As in the trigger, a cluster is one or two towers adjacent in $\eta$. The ratio of hadronic to electromagnetic energy in the cluster must fall within the range $E_{\text {had }} / E_{\text {em }}<$ $0.055+0.00045 E_{t o t}$, where the energy dependence acknowledges that higher energy electrons are more likely to produce showers that extend into the hadronic calorimeter. The cluster must also be isolated, that is the $E_{T}$ deposited in a cone of $\Delta R=\sqrt{(\Delta \eta)^{2}+(\Delta \phi)^{2}} \leq 0.4$ around the cluster must be less than $10 \%$ of the cluster $E_{T}$. We require a good threedimensional COT track [57] that points to the EM cluster. It must contain at least three good axial track segments and two good stereo segments, each with at least five hits. The track must point to a fiducial region of the calorimeter: to an instrumented region and away from the edges of towers. The track's vertex, where it crosses the beamline, must be within $60.0 \mathrm{~cm}$ of the center of the CDF detector to guarantee high COT reconstruction efficiency - this is, anyhow, the expected luminous region. The track $p_{T}$ must be at least $10 \mathrm{GeV}$ and the ratio $E / p$ of cluster energy to track momentum must be less than 2.0, allowing some latitude for radiated bremsstrahlung photons which reduce the track momentum but likely deposit energy in the same EM cluster. This requirement is removed for track $p_{T}>50$ $\mathrm{GeV}$.

Photon conversions are identified by searching for opposite sign COT tracks with trajectories consistent with the conversion hypothesis. If a candidate electron is part of such a conversion pair it is rejected. But, if a third track also satisfies the conversion definition then the likely history is a high-energy bremsstrahlung photon emitted from the original electron, which then converts into an electron-positron pair (a "trident" electron). So, the original particle was likely an electron, and is accepted as such.

We require a cluster in the CES shower-max detector whose position agrees with the extrapolated track position. Its position must agree to within $2.0 \mathrm{~cm}$ in $z$ and must satisfy $-3.0<Q \times \Delta x<1.5 \mathrm{~cm}$ where $\mathrm{Q}$ is the charge of the track and $\Delta x$ is the separation in the $r-\phi$ plane between the CES cluster centroid and the extrapolated track position. This asymmetric cut is again to accommodate photonic radiation, which does not bend in the magnetic field and so distorts the position of the CES cluster from what we would expect from the electron (or positron) track alone. And, we require that the CES shower profile and the lateral sharing of energy between calorimeter towers in the EM cluster must 
agree with expectations based on test beam electrons.

The electron energy is taken from the EM cluster energy with small corrections (i.e. face corrections) applied depending on where in the calorimeter tower the electron enters. The mass of the electron is set to zero and the angles for the momentum components are taken from the COT track.

\subsubsection{Offline Muon Reconstruction and Selection}

Offline muon reconstruction begins with a good three-dimensional COT track and corresponding hits in the CMX or CMU and CMP muon chambers (a muon "stub"). The COT track has the same basic requirements as in the electron case, but the $p_{T}$ must be greater than $20 \mathrm{GeV} .|\Delta x|$ between the muon stub and the extrapolated COT track must be less than $3.0 \mathrm{~cm}$ in the $\mathrm{CMU}$ and $5.0 \mathrm{~cm}$ in the CMP or less than $6.0 \mathrm{~cm}$ in the CMX. As a fiduciality requirement, the exit radius of a muon track from the COT must be greater than $140 \mathrm{~cm}$ to guarantee that the muon passes through all four axial layers of the COT and falls within the XFT acceptance. To eliminate muons resulting from decays-in-flight of heavier particles the impact parameter of the muon track must be less than $0.2 \mathrm{~cm}$, or less than $0.02 \mathrm{~cm}$ if there are silicon hits attached to the COT tracks providing improved impact parameter resolution. Energy deposited in the calorimeter along the trajectory of the muon candidate is require to be consistent with a minimum ionizing particle, and muons identified as possibly coming from cosmic rays are vetoed. Cosmic ray candidates are identified by comparing the track beamline crossing time with the actual beam crossing and by checking consistency with a back-to-back track hypothesis. Muon candidates are also required to be isolated, that is the $E_{T}$ deposited in a cone of $\Delta R=\sqrt{(\Delta \eta)^{2}+(\Delta \phi)^{2}} \leq 0.4$ around the muon must be less than $10 \%$ of the muon $p_{T}$. A small correction to the muon momentum is applied (curvature corrections) due to chamber misalignment.

\subsection{Jet Reconstruction and Corrections}

Strongly interacting particles produced in high-energy interactions, quarks and gluons, do not interact directly in our detector. Instead, they quickly fragment and hadronize into a collimated group of pions, kaons, and other hadrons (a "jet") which then interacts in the the detector. The charged members of this group interact interact in the tracking systems and then the entire group deposits its energy across generally a large number of 
towers in the electromagnetic and hadronic calorimeters. To measure the top mass we would like to know the momentum four-vector of the original quark. We begin by clustering the energy in the calorimeter into a reconstructed jet and then apply a series of corrections to move back towards parton level. In parallel, we use the tracking information from the jet to identify interaction vertices displaced from the primary event vertex, evidence of a jet originating from a $b$ quark.

\subsubsection{Jet Clustering}

This analysis uses the JETCLU clustering algorithm, with a cone of radius $R=$ $\sqrt{(\Delta \eta)^{2}+(\Delta \phi)^{2}}=0.4$, in a way similar to what was used at CDF in Run 1 [58]. The algorithm begins with a list of seed towers, towers with $E_{T}>1.0 \mathrm{GeV}$, defined in order of decreasing $E_{T}$. Clusters are formed from these seed towers by including all towers with energy of at least $100 \mathrm{MeV}$ whose centers are within a $R=0.4$ from the seed tower.

The clusters then evolve through an iterative procedure. The cluster $E_{T}, \phi$, and $\eta$ are calculated:

$$
\begin{gathered}
E_{T}^{\text {cluster }}=\sum_{i=0}^{N_{\text {tow }}} E_{T i} \\
\phi^{\text {cluster }}=\sum_{i=0}^{N_{\text {tow }}} \frac{E_{T i} \phi_{i}}{E_{T}^{\text {cluster }}} \\
\eta^{\text {cluster }}=\sum_{i=0}^{N_{\text {tow }}} \frac{E_{T i} \eta_{i}}{E_{T}^{\text {cluster }}}
\end{gathered}
$$

where $N_{\text {tow }}$ is the number of towers having energy of at least $100 \mathrm{MeV}$ and within a radius of $R=0.4$ of the previous iteration's cluster centroid (the cluster centroid for the first iteration is the seed tower itself). This is repeated iteratively until the list of towers in the cluster is stable. Then, clusters are merged if they overlap significantly or otherwise they are split apart. The final products of the clustering are our reconstructed jets. Ultimately, a tower can be present in only one jet.

The energy of the jet is a scalar sum of tower energies

$$
E_{j e t}=\sum_{i=0}^{N_{\text {tow }}} E_{i}
$$


while the momentum of the jet is a scalar sum:

$$
\begin{gathered}
p_{x}^{j e t}=\sum_{i=0}^{N_{\text {tow }}} E_{i} \sin \left(\theta_{i}\right) \cos \left(\phi_{i}\right) \\
p_{y}^{\text {jet }}=\sum_{i=0}^{N_{\text {tow }}} E_{i} \sin \left(\theta_{i}\right) \sin \left(\phi_{i}\right) \\
p_{z}^{\text {jet }}=\sum_{i=0}^{N_{\text {tow }}} E_{i} \cos \left(\theta_{i}\right)
\end{gathered}
$$

The angular position of the jet is determine from the momentum components, and the transverse momentum and energy are calculated based on these angles. This algorithm will form jets around our electron candidates also, but these jets are removed.

\subsubsection{Jet Energy Corrections}

To connect the measured jet energy, as just described, to the original parton energy of the quarks from $t \bar{t}$ decay we use two sets of tools. The first are the generic jet corrections which we use in this analysis to take us from calorimeter jets back to the jets of particles before interaction in the calorimeter. That is, we use them to remove all detector effects. The last stage, the mapping between parton level and particle jet level, will be handled by $t \bar{t}$-specific transfer functions and described in Chapter 6 . We will describe the generic corrections briefly - they, and the jet energy scale at CDF generally, are described in detail elsewhere [59].

The first corrections to be applied remove the $\eta$-dependence of the calorimeter with respect to the well understood central region. This is especially important where there are significant non-uniformities or uninstrumented regions, such as between the two halves of the central calorimeter, or between the central, wall, and plug calorimeters. These relative corrections are extracted from samples of di-jet events, over a wide range of energies, from data and PYTHIA MC. They rely on the fact that in an event with only two jets, their transverse energies should balance. The $p_{T}$ of a "probe" jet, anywhere in the calorimeter is compared to the $p_{T}$ of a "trigger" in the central region, away from uninstrumented regions, $0.2<|\eta|<0.6$. Results from one data and MC sample can be seen in Figure 4.2. They agree well up to $\eta=2.0$ but different corrections are extracted for data and MC. The final corrections are spline-interpreted continuous functions of $p_{T}$ and binned functions of $\eta$. 


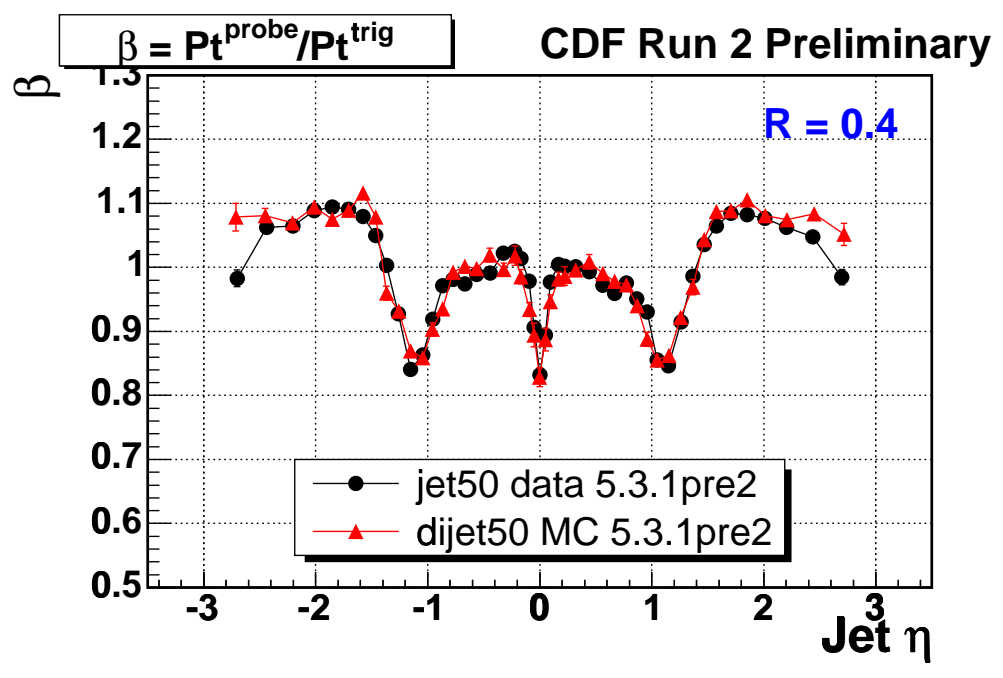

Figure 4.2: Di-jet balancing for PYTHIA MC and data with a $50 \mathrm{GeV}$ jet trigger. The probe jet is a central jet away from uninstrumented regions, $0.2<|\eta|<0.6$ while the probe jet may be anywhere in the calorimeter.

The next correction is to correct for multiple $p \bar{p}$ interactions that can occur in the same bunch crossing. Energy from such interactions can be deposited within the cone of a jet from a $t \bar{t}$ event. An average correction is determined by studying the amount of energy deposited in a randomly chosen cone of radius 0.4 in a minimum bias event, an event triggered simply by requiring hits in the CLC counters on either side of the detector. The correction is parameterized on the number of primary vertices observed in an event, and amounts to $0.36 \mathrm{GeV}$ per additional vertex.

The last generic correction used in this analysis is the so-called absolute correction. It accounts for the non-linear response of the calorimeter and for the various ways energy in a jet can be shared among its constituent particles. The CDF calorimeters respond differently to particles of various energies, and the energy spectrum of particles in a jet varies event by event. One $50 \mathrm{GeV} b$ quark could fragment into a jet where most of the energy goes to a leading hadron, while a second $50 \mathrm{GeV} b$ quark might share its energy more democratically. An average correction is determined from di-jet $\mathrm{MC}$ corrected to a flat $p_{T}$ spectrum. This correction relies on the careful tuning of the detector simulation, based on in situ calibrations from data tracks at low energies and on test beam data at high energies, and on a well-modelled MC fragmentation process. This correction is a function of $p_{T}$ only and can be seen in Figure 4.4. 


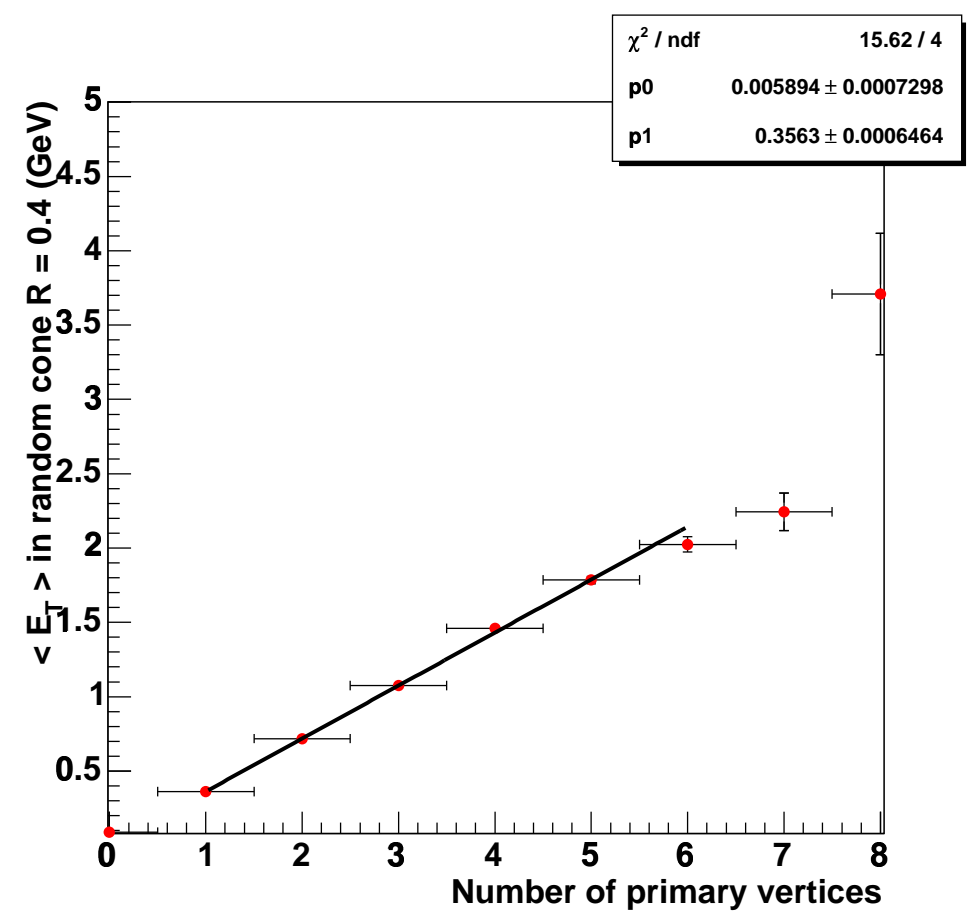

Figure 4.3: Energy in a random cone, radius 0.4, in minimum bias events. This function, parameterized on the number of primary vertices in the event, is used to correct for the effects of multiple $p \bar{p}$ interactions in one bunch crossing.

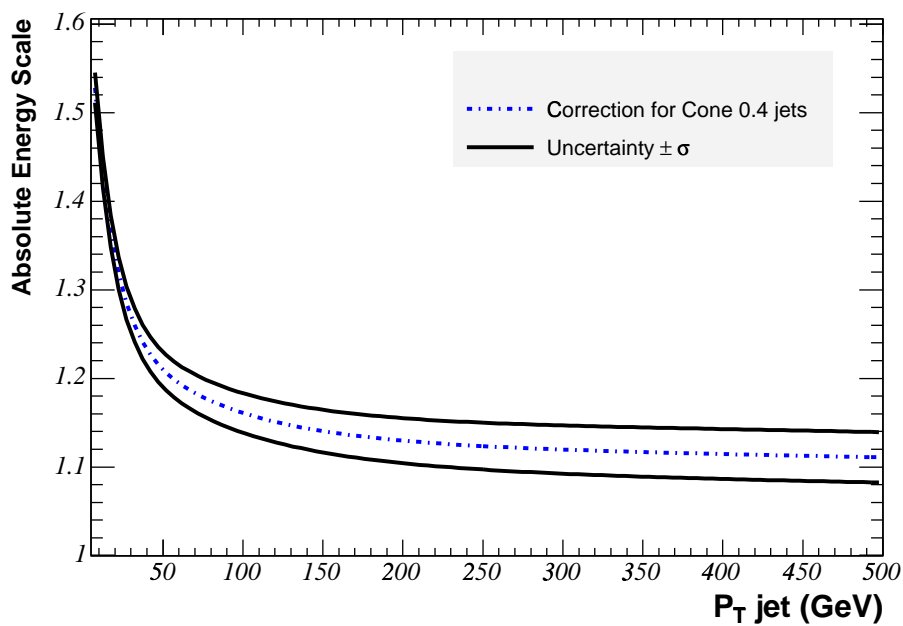

Figure 4.4: The absolute energy scale correction as a function of jet $p_{T}$. This factor corrects, on average, for calorimeter non-linearities and fragmentation effects. 
Generic corrections are also available to correct for portions of the original $p \bar{p}$ system not involved in the hard-scattering event (i.e. the underlying event) and to correct for parton energy that does not fall within the cone of our clustering algorithm. In this analysis these effects are absorbed into our transfer functions. The systematic uncertainties on these corrections will be relevant, but will be described along with all other systematic uncertainties in a later chapter.

\subsubsection{Displaced Vertex $b$-Tagging}

Since every top quark decay results in a $b$ quark, identifying events with $b$ quarks is a helpful way to obtain a pure sample of $t \bar{t}$ events; it also reduces the number of jet-parton assignments that must be considered when reconstructing the mass of the top quark. $B$ hadrons, with a mean lifetime of about $1.4 \mathrm{ps}$, are highly boosted in $t \bar{t}$ decays and can travel millimeters before decaying, often far enough to be observed as secondary vertices with the silicon vertex detector.

We use the "tight" version of the SecVtx algorithm which considers jets with uncorrected $E_{T}>15 \mathrm{GeV}$. The algorithm begins by considering good quality tracks, with both COT and silicon hits, inside the jet. If two such tracks are found that reconstruct to a common secondary vertex the distance $\left(L_{x y}\right)$ between the primary and secondary vertices in the transverse $(r-\phi)$ plane is considered. $L_{x y}$ is a signed distance, with a negative $L_{x y}$ indicating a vertex displaced in the opposite direction from the jet's momentum. These negative $L_{x y}$ 's are indicative of backgrounds and/or CDF's vertex resolution. If the secondary vertex is significantly displaced from the primary event vertex (we require $L_{x y} / \sigma\left(L_{x y}\right)>7.5$ ) then the jet is considered tagged. The tagging efficiency as a function of jet $E_{T}$ is shown in Figure 4.5. The tagging efficiency also depends significantly on $\eta$ as also seen in Figure 4.5, dropping off significantly for $|\eta|>1.0$ as the silicon and especially the COT become less efficient. These are the efficiencies for tagging a particular $b$ jet in a $t \bar{t}$ event, about $50 \%$ for jets with $|\eta|<1.0$ and $E_{T}>40 \mathrm{GeV}$. The efficiency to tag at least one $b$ jet in a lepton + jets $t \bar{t}$ event is about $60 \%$. It is also possible to mistakenly tag a light quark jet. The SecVtx algorithm mistags light quark jets with an $E_{T}$-dependent probability of about $1 \%$ in $t \bar{t}$ events. More details on displaced vertex $b$ tagging are available elsewhere [56]. 

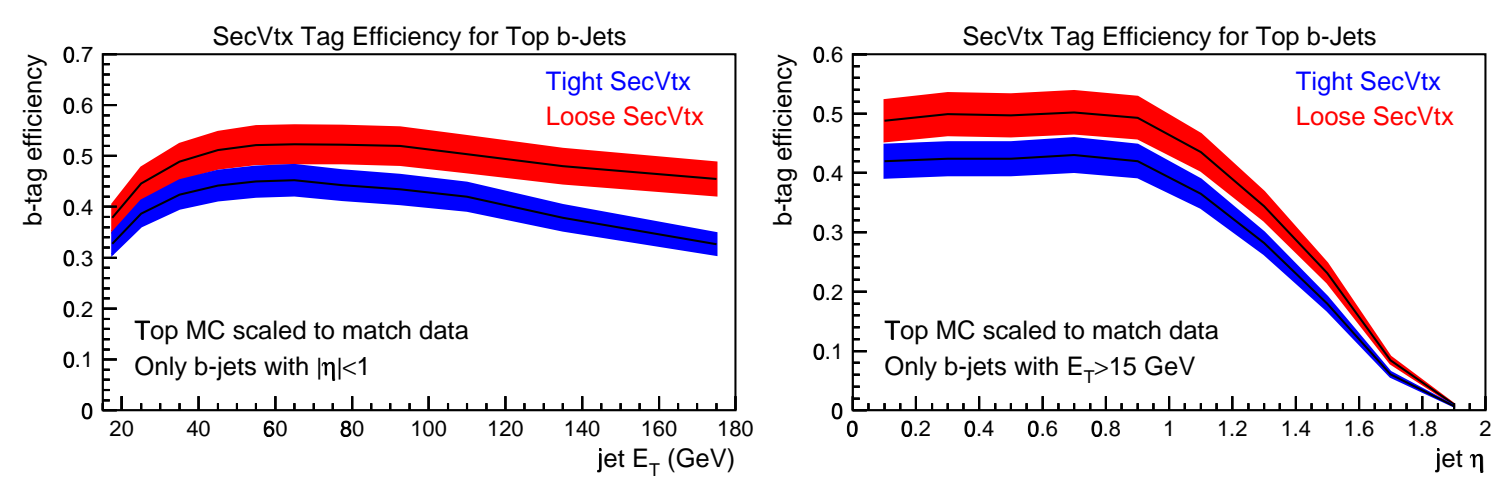

Figure 4.5: The efficiency to tag a particular $b$ jet in $t \bar{t}$ events with the SecVtx algorithm.

\subsection{Missing Transverse Energy}

If we assume that the initial transverse momentum of the incoming partons in $t \bar{t}$ production is zero - that is that the quarks or gluons responsible for the hard scattering have momenta only along the beamline, i.e. in the $z$ direction - then we may interpret any imbalance of transverse energy in the detector as evidence for a neutrino. And, we may interpret the magnitude of such a transverse energy imbalance as the $p_{T}$ of the neutrino.

We define the missing transverse energy as

$$
E_{T}=-\left|\sum_{i} E_{T}^{i} \hat{n}_{i}\right|
$$

where the sum is over all towers in the central, wall, and hadronic calorimeters and where $\hat{n}_{i}$ is the unit vector pointing from the event vertex to the $i$ th calorimeter tower, which has transverse energy $E_{T}^{i}$. Since muons deposit little energy in the calorimeter, for events with a muon we correct the $\mathbb{E}_{T}$ by inserting the corrected muon momentum into the above equation and removing the corresponding calorimeter towers. An improved estimate of the energy in jets is also available, as we have described in Section 4.4.2. So we also correct Equation 4.8 by adding the transverse energy of jets, corrected for response relative to the central calorimeter and for multiple interactions, to the sum and removing the associated calorimeter towers. For an event to enter our $t \bar{t}$ sample we require that the corrected $\mathbb{E}_{T}$ be greater than $20 \mathrm{GeV}$. As we will describe in detail later, we do not use this corrected $\mathbb{E}_{T}$ variable directly in our mass reconstruction. Instead we calculate the neutrino momentum for each point in phase space using the corrected lepton momentum and the parton momenta of that point in phase space. 


\subsection{Summary of Event Selection Requirements}

So, with those definitions behind us, our basic event selection is to require exactly four jets with corrected $E_{T}>15.0 \mathrm{GeV}$ and $|\eta|<2.0$, one central electron (or muon) with corrected $E_{T}>20 \mathrm{GeV}\left(p_{T}>20 \mathrm{GeV} / c\right)$, and large corrected $\mathbb{E}_{T}\left(\mathscr{E}_{T}>20 \mathrm{GeV}\right)$. We also require at least one of the jets to have a tight secondary vertex $b$ tag as evidence of a $b$ quark.

The full event selection criteria contain a few other details of a global nature. We allow events that have the above-mentioned four jets (four "tight" jets) and also contain additional jets that satisfy looser criteria ("loose" jets). Only the four tight jets are used for mass reconstruction and we veto events with more than four tight jets. To help guarantee consistency with our lepton + jets hypothesis, and to make combinations of $t \bar{t}$ measurements across channels easier, we reject events which satisfy the selection criteria used for dilepton $t \bar{t}$ analyses at CDF. That is, we reject events which have one primary (or "tight" lepton, as described in sections 4.3.1 and 4.3.2) and one secondary ("tight", or "loose", satisfying looser quality requirements or observed outside the central region of the det

We require that the lepton track is consistent with the event vertex. That is, we require that the $z$ coordinate of the event vertex corresponds to the lepton $z_{0}$ to within 5.0 $\mathrm{cm}$. The lepton $z_{0}$ is the $z$ coordinate of the point of closest approach of the lepton track to the origin in the $x-y$ plane $(x=y=0)$. We also require that the event vertex is within the fiducial and luminous region of the CDF detector, $|z|<60 \mathrm{~cm}$.

Most of the event selection criteria so far described are common to many high- $p_{T}$ analyses at CDF, and especially to lepton + jets $t \bar{t}$ analyses. There is one additional event selection parameter, unique to our analysis, which will be described in detail later. We veto events that exhibit a certain pathological behavior when processed by our $t \bar{t}$ signal probability. We will refer to this one additional event selection requirement as the $t \bar{t}$ signal probability, or $P_{t \bar{t}}$, cut.

\subsection{Events Observed in Data}

The overall acceptance of $t \bar{t}$ events, the probability for a $t \bar{t}$ event to pass the above event selection requirements, is about $2 \%$. In $318 \mathrm{pb}^{-1}$ of data collected from March 2002 to August 2004 we observe 63 events that satisfy these selection requirements. This represents 
Tevatron stores between 1120 and 3745. The data acquisition system at CDF subdivides data into "runs" and this dataset represents runs betweeen 141544 and 186598. The 318 $\mathrm{pb}^{-1}$ represents runs where all relevant subdetectors are delivering data of good quality. CMX muons are only available for a portion of this dataset, runs after 150145 representing a luminosity of approximately $305 \mathrm{pb}^{-1}$. The events are listed in Tables 4.1 and 4.2. There are a total of $39 \mathrm{CEM}$ electron candidate events, 18 CMUP muon candidates, and $6 \mathrm{CMX}$ muon candidates. 47 of the events have on SecVtx tagged $b$ jet and 16 events have two $b$ tags.

\subsection{Background Estimates and Modelling}

The $318 \mathrm{pb}^{-} 1 t \bar{t}$ data set that we use for our analysis, based on the event selection requirements we have described, is perhaps more honestly referred to as a $W+$ jets sample. Or, with the requirement of a displaced-vertex $b$ tag, a $W+$ heavy flavor sample. There are a number of other physics processes that can contribute events to the same sample. Some of these are relatively rare processes fairly similar to the lepton + jets $t \bar{t}$ events of interest, while others are not nearly so similar to $t \bar{t}$ but are very abundant. Both broad categories of events can contribute comparable numbers of background events.

These background events are problematic primarily for two reasons. First, the matrix elements and associated signal and background probabilities with which we measure the mass of the top quark only explicitly model leading-order $t \bar{t}$ and $W+$ heavy flavor events. Events of any other type at best contribute noise to our measurement. They may reduce our sensitivity to the top mass, may affect the accuracy of our error estimate, and may even bias our mass measurement. In particular, many of the backgrounds are kinematically similar to $t \bar{t}$ events with a smaller top mass than has so far been measured experimentally. Since these events could potentially bias our mass measurement it is important to understand their frequency and to be able to model their effect on our analysis.

The backgrounds that we explicitly consider can be divided into four categories:

$W+$ Jets, Non- $W$ QCD, electroweak, and single top. The $W+$ Jets background consists of events with a real $W$ boson, which decays to an electron or a muon. At least four associated jets are also produced, which include either a legitimately tagged $b$ or $c$ jet or a light-flavor jet mistagged by the SecVtx algorithm. The non- $W$ QCD background consists of events with no electroweak production at all, containing only a large number (five or more) of jets. 


\begin{tabular}{|c|c|c|c|}
\hline Run Number & Event Number & $\# b$ tags & Lepton Type \\
\hline 153694 & 1694029 & 2 & CEM \\
\hline 153738 & 205803 & 2 & CEM \\
\hline 153738 & 2083102 & 1 & CEM \\
\hline 154175 & 1630925 & 1 & CEM \\
\hline 155320 & 480816 & 1 & CEM \\
\hline 155919 & 2689969 & 1 & CEM \\
\hline 156457 & 13182 & 1 & CEM \\
\hline 160153 & 1270879 & 1 & CEM \\
\hline 160230 & 805211 & 2 & CEM \\
\hline 160441 & 3910866 & 1 & CEM \\
\hline 160594 & 290458 & 1 & CEM \\
\hline 161633 & 1571961 & 1 & CEM \\
\hline 161792 & 391660 & 1 & CEM \\
\hline 162423 & 261933 & 1 & CEM \\
\hline 164110 & 954852 & 2 & CEM \\
\hline 164274 & 1449940 & 1 & CEM \\
\hline 164819 & 1242550 & 1 & CEM \\
\hline 165314 & 236898 & 1 & CEM \\
\hline 166614 & 804529 & 2 & CEM \\
\hline 166653 & 1499964 & 2 & CEM \\
\hline 166715 & 357810 & 1 & CEM \\
\hline 166717 & 3530653 & 1 & CEM \\
\hline 167053 & 12401969 & 1 & CEM \\
\hline 168563 & 2395692 & 1 & CEM \\
\hline 177314 & 2950396 & 1 & CEM \\
\hline 177345 & 3135596 & 1 & CEM \\
\hline 178258 & 782935 & 1 & CEM \\
\hline 178677 & 4378990 & 1 & CEM \\
\hline 183209 & 1059754 & 1 & CEM \\
\hline 183631 & 183631 & 1 & CEM \\
\hline 183752 & 3562502 & 1 & CEM \\
\hline 184419 & 291129 & 1 & CEM \\
\hline 184453 & 19917 & 1 & CEM \\
\hline 184782 & 2170277 & 1 & CEM \\
\hline 185075 & 4388549 & 1 & CEM \\
\hline 185332 & 1622825 & 1 & CEM \\
\hline 185349 & 57399 & 2 & CEM \\
\hline 185777 & 5392044 & 1 & CEM \\
\hline 186145 & 9795252 & 1 & CEM \\
\hline
\end{tabular}

Table 4.1: $t \bar{t}$ electron candidate events observed in $318 \mathrm{pb}^{-1}$ of data collected from March 2002 to August 2004. 


\begin{tabular}{|c|c|c|c|}
\hline Run Number & Event Number & $\# b$ tags & Lepton Type \\
\hline 145036 & 245760 & 1 & CMUP \\
\hline 152266 & 3554 & 1 & CMUP \\
\hline 153693 & 799494 & 2 & CMUP \\
\hline 160437 & 280173 & 1 & CMUP \\
\hline 161788 & 361577 & 2 & CMUP \\
\hline 162837 & 921871 & 1 & CMUP \\
\hline 163012 & 2249546 & 1 & CMUP \\
\hline 166529 & 4938 & 1 & CMUP \\
\hline 166567 & 11615607 & 1 & CMUP \\
\hline 166717 & 2288892 & 2 & CMUP \\
\hline 166805 & 2534588 & 1 & CMUP \\
\hline 168889 & 1456443 & 1 & CMUP \\
\hline 178120 & 86683 & 1 & CMUP \\
\hline 178855 & 5504617 & 2 & CMUP \\
\hline 185248 & 8569330 & 2 & CMUP \\
\hline 185332 & 4430084 & 1 & CMUP \\
\hline 185518 & 330101 & 1 & CMUP \\
\hline 186087 & 17361 & 2 & CMUP \\
\hline \hline 154654 & 6534372 & 1 & CMX \\
\hline 166367 & 516271 & 2 & CMX \\
\hline 167139 & 1191211 & 2 & CMX \\
\hline 178785 & 1428968 & 1 & CMX \\
\hline 179039 & 2128943 & 2 & CMX \\
\hline 184832 & 12978334 & 1 & CMX \\
\hline
\end{tabular}

Table 4.2: $t \bar{t}$ muon candidate events observed in $318 \mathrm{pb}^{-1}$ of data collected from March 2002 to August 2004. 
One of these jets is mis-identified as an electron or a muon, one obtains a real or fake SecVtx tag, and a combination of jet $E_{T}$ mismeasurements results in large $\mathbb{E}_{T}$. The electroweak background consists of $W W, W Z$, or $Z Z$ events with additional jets. The electroweak bosons provide the leptons (and, in the case of $W W$ and $W Z, \mathbb{E}_{T}$ ) and some of the jets. This, along with additional jets, fills out the event selection requirements, including a real or fake SecVtx tag (and, for the $Z Z$ events, fake $\mathbb{E}_{T}$ ). The single top background consists of isolated top quarks from electroweak production, either in the $s$ or the $t$ channel. The top decay provides a $b$ quark, a lepton, and $\mathbb{E}_{T}$, meaning that only three additional jets are needed to satisfy our event selection criteria.

The number of background events expected in each of these processes is estimated with a combination of information from data, from Monte Carlo studies, and from theoretical inputs such as the cross sections of the electroweak processes. For each background we also need a source of events for use in studies of our mass analysis techniques in simulated experiments (pseudo-experiments). These events come from various Monte Carlo event generators and, in the case of the non- $W$ QCD events, from data. The process of determining the background estimates and obtaining events for pseudo-experiments will be described, briefly, below.

As we have mentioned, the event selection requirements are similar for most lepton + jets $t \bar{t}$ analyses at CDF. We have taken special care to ensure that our event selection is compatible with one particular lepton + jets $t \bar{t}$ cross section measurement [60], from which we draw our background estimates. It is common at CDF for cross section analyses, where a detailed understanding of backgrounds is of first importance, to make a careful study of backgrounds and for mass analyses to take advantage of their study. We summarize their results below, but full details are available elsewhere [60]. As we discuss later, we consider the uncertainty on the normalization and composition of the backgrounds as a source of systematic error.

Apart from our $P_{t \bar{t}}$ cut, the event selection requirements of the two analyses are identical. Our event selection has also been confirmed to be identical to the CDF top mass measurement using the Dynamical Likelihood Method [26, 27]. The comparison with the cross section analyses is with the one or more $b$ tag, exactly four jet, version of their "unoptimized" 1 analysis.

\footnotetext{
${ }^{1}$ For their main result they attempted to minimize the statistical uncertainty on $\sigma_{t \bar{t}}$ by maximizing $\frac{S}{\sqrt{S+B}}$, where $\mathrm{S}$ and $\mathrm{B}$ are the number of signal and background events, respectively, in the sample. Their
} 


\begin{tabular}{|c|c|c|c|}
\hline & $\begin{array}{c}\text { Expected Events } \\
\text { Before } P_{t \bar{t}} \text { Cut }\end{array}$ & $\begin{array}{c}\text { Fraction Passing } \\
P_{t \bar{t}} \text { Cut }\end{array}$ & $\begin{array}{c}\text { Expected Events } \\
\text { After } P_{t \bar{t}} \text { Cut }\end{array}$ \\
\hline$W+4 p$ (Mistags) & $2.23 \pm 0.44$ & $0.9458 \pm 0.0040$ & $2.11 \pm 0.42$ \\
\hline$W b \bar{b}$ & $1.70 \pm 0.80$ & $0.9342 \pm 0.0080$ & $1.59 \pm 0.75$ \\
\hline Non-W (QCD) & $3.07 \pm 1.06$ & $0.960 \pm 0.022$ & $2.95 \pm 1.02$ \\
\hline$W c \bar{c}$ & $0.81 \pm 0.40$ & $0.939 \pm 0.014$ & $0.76 \pm 0.37$ \\
\hline Wc & $0.51 \pm 0.23$ & $0.965 \pm 0.012$ & $0.49 \pm 0.22$ \\
\hline Single Top & $0.41 \pm 0.09$ & $0.9710 \pm 0.0064$ & $0.40 \pm 0.09$ \\
\hline EW (WW, WZ, ZZ) & $0.39 \pm 0.08$ & $0.955 \pm 0.022$ & $0.37 \pm 0.08$ \\
\hline \hline Total Background & $9.12 \pm 1.83$ & $0.9477 \pm 0.0030$ & $8.67 \pm 1.74$ \\
\hline \hline$t \bar{t}(6.1$ pb) & $36.86 \pm 3.80$ & $0.9751 \pm 0.0024$ & $35.94 \pm 3.71$ \\
\hline \hline & Observed Events & & Observed Events \\
& Before $P_{t \bar{t}}$ Cut & & After $P_{t \bar{t}}$ Cut \\
\hline Data & 63 & & 63 \\
\hline
\end{tabular}

Table 4.3: Estimated number of background events in our dataset and expected number of $t \bar{t}$ events for $\sigma_{t \bar{t}}=6.1 \mathrm{pb}$. Estimates before $P_{t \bar{t}}$ cut are from a $t \bar{t}$ cross section measurement with the same event selection requirements [60]. They measure $\sigma_{t \bar{t}}=8.9 \mathrm{pb}$ with these event selection requirements.

Their background estimates are shown as the second column of Table $4.3^{2}$. We pass simulated $t \bar{t}$ events, and events representing each of the background types, through our signal probability to estimate the fraction passing the $P_{t \bar{t}}$ cut and use it to update the expected number of events. We will describe the original background estimates briefly, below. More details are available elsewhere [60]. And, once we have described the $t \bar{t}$ signal probability, we will describe the $P_{t \bar{t}}$ cut in more detail.

\subsection{1 $W+$ Jets}

The $W+$ Jets background is considered as two separate populations, those events with real heavy flavor jets, and those with light jets misidentified as containing heavy flavor. Monte Carlo based predictions are available for these processes, but only in the leading order. These predictions, in the form of ALPGEN-generated events with parton

"optimized" choice of event selection added two cuts: $H_{T}>200 \mathrm{GeV}$ (the scalar sum of transverse energies in the event) and $M_{T}^{W}>20 \mathrm{GeV}$ (the transverse mass of the leptonic $W$ boson). As with most lepton + jets cross section measurements they used events with three or more jets.

${ }^{2}$ It has lately come to our attention that three small changes have been made to the background estimates. The most significant of these is the number of mistagged $\mathrm{W}+$ light jets events. We use $2.23 \pm 0.44$ while their revised estimate is $2.27 \pm 0.45$. Since the magnitude of the change is so small compared to the estimated error, which we count as a systematic uncertainty on the top mass, we have not updated our result. 
showering and hadronization provided by HERWIG, are one input to the calculation. But, because of inadequacies in the event generators (such as imprecise values of heavy flavor fractions) and in our detector simulation (such as the imperfect description of our tracking systems leading to incorrect values for $b$ tagging efficiencies) it is necessary to incorporate results from data as well.

\section{$W+$ Heavy Flavor}

The $W+$ heavy flavor background consists of events with a real $W$ boson with associated jets and, typically, a pair of $b$ or $c$ quarks produced by the splitting of a gluon. Our estimate of the number of such events begins by considering the number of $W+$ Jets events in data, before requiring a $b$ tag, and then subtracting the number of events we expect from other backgrounds (non- $W$ QCD, EW, and single top) and the number of expected $t \bar{t}$ events. ${ }^{3}$ This corrected number of events is then multiplied by the heavy flavor fraction (the number of events expected to contain heavy flavor), by the event tagging efficiency from Monte Carlo, and by a scale factor to correct this event tagging efficiency to appropriately describe data.

The event tagging efficiency, the probability of the SecVtx algorithm tagging an event which legitimately contains heavy flavor, is evaluated with $W+$ heavy flavor ALPGEN + HERWIG Monte Carlo samples. ALPGEN produces $W$ events with a known number of additional partons in the matrix element, and HERWIG may then add additional jets from initial and final-state radiation. So, a matching algorithm is employed to correctly combine various ALPGEN + HERWIG samples into an appropriate $W+4$ Jet sample. This event tagging efficiency must be corrected by a scale factor which accounts for the differences in event tagging rates in data and Monte Carlo. This event tagging scale factor is a combinatoric factor that takes into account the efficiency of tagging individual $b$ and $c$ jets, the efficiency of mistagging light jets, and the data-Monte Carlo scale factors to correct these individual jet tagging rates. The individual jet scale factors are measured by comparing data tagging efficiencies, as measured in heavy flavor enriched di-jet data samples, to Monte Carlo expectations.

The heavy flavor fraction is measured in a multi-step process, by calibrating the

\footnotetext{
${ }^{3}$ The number of $t \bar{t}$ events subtracted is the number expected based on the measured cross section, which is determined based upon these same background calculations. So, the top cross section measurement is an iterative process, repeated until the cross section converges.
} 
heavy flavor fraction in ALPGEN in a control data set and then applying this calibration to ALPGEN heavy flavor predictions for $W+$ Jets events. The calibration begins by forming templates, from ALPGEN di-jet Monte Carlo, of the $c \tau$ distribution of $b, c$, and light flavor jets. These templates are then compared to the $c \tau$ distribution of tagged jets in data and the fraction of tagged jets with $b, c$, and light flavor content is determined. Then, we divide by the tagging efficiency to obtain the heavy flavor fraction in the pre-tagged sample. The heavy flavor fraction is also determined directly in Monte Carlo. We take the ratio of pre-tag heavy flavor fractions, in di-jet events, in data and Monte Carlo and treat it as a heavy flavor scale factor. Then, we measure the heavy flavor fraction in ALPGEN $W+$ Jets events, and multiply by the data-Monte Carlo heavy flavor scale factor to obtain our final heavy flavor fraction. This final $W+$ Jets heavy flavor fraction is used to predict the number of expected $W+$ heavy flavor events in our data set.

With the predicted number of $W+$ heavy flavor events in hand, we still need a source of simulated events to use in pseudo-experiments in order to test our analysis machinery for the top mass measurement. We use a variety of ALPGEN + HERWIG Monte Carlo samples and apply our full event selection, exactly as it is used on the actual data. The samples include $W b \bar{b}+2$ partons, $W c \bar{c}+2$ partons, and $W c+3$ partons.

\section{$W+$ Light Flavor, Mistags}

The $W+$ Light Flavor background consists of events with a real $W$ boson with associated light $(u, c$, or $s)$ jets where one of the light jets is mistakenly tagged as a $b$ jet. To estimate the number of such events in our data sample, we begin with the number of pre-tagged $W+$ Jets events. We first correct for the number of events due to other backgrounds and the number of expected events from $t \bar{t}$. Then, we apply a negative tag, or mistag, matrix which gives the expected rate of mistagging light quarks as a function of $E_{T}$. Finally, we apply an asymmetry factor to correct the results of the mistag matrix.

The mistag matrix parameterizes those fake tags due to CDF's tracking resolution. Consider a promptly decaying hadron, whose decay vertex should be consistent with the primary event vertex. Because of the resolution of CDF's tracking systems a vertex reconstructed from tracks in this jet will lie somewhere within an error ellipse. At the extremes of this ellipse, a light jet may satisfy the requirements of the SecVtx algorithm and be declared "tagged". To measure the frequency of this occurrence a sample of di-jet 
events from data, not enriched in heavy flavor, is considered. Those jets with negative $L_{x y}$ (i.e. whose vertices are on the side of the primary vertex opposite to what we would expect from the jet's momentum) are considered to be mistagged. Since these mistags are assumed to result from a Gaussian (and hence symmetric) tracking resolution distribution our first assumption is that we will have an equal number of mistagged jets with positive $L_{x y}$.

However, we do not expect the distribution of $L_{x y}$ values for light jets to be completely symmetric. In particular, we expect certain long-lived hadrons, such as $\Lambda$ and $K_{0}^{s}$, to be present in light jets. These tend to cause a long positive tail in the $L_{x y}$ distribution of light jets. So, a correction for this asymmetry is necessary. This factor is calculated in a manner similar to the heavy flavor fractions, described above. Templates of the $c \tau$ distributions of $b, c$, and light jets are formed from di-jet MC samples and fit to corresponding samples from data. From this, an $E_{T}$-dependent asymmetry factor is extracted.

For our pseudo-experiments we use $W+4$ parton events from ALPGEN + HERWIG. We apply our entire event selection regimen, as used in data, with one exception. We do not require a SecVtx tag as this requirement would require generating a prohibitively large number of light flavor events. In any case, we do not expect our simulation to accurately represent mistagged $W+$ light flavor events, and so would expect no appreciable improvement in fidelity for such laboriously simulated events.

\subsubsection{Electroweak and Single Top}

The expected numbers of events from electroweak ( $W W, W Z$, and $Z Z$ ) and single top backgrounds are calculated based upon theoretical cross sections with acceptances and efficiencies calculated in Monte Carlo and modified with corrections from data. These backgrounds contribute real leptons and (except for $Z Z$ ) real $\mathbb{E}_{T}$. They require additional jets from initial or final-state radiation, and of course must have a real or fake $b$ tag. The cross sections used are: $p \bar{p} \rightarrow W W 13.25 \pm 0.25 \mathrm{pb}, p \bar{p} \rightarrow W W 3.96 \pm 0.06 \mathrm{pb}, p \bar{p} \rightarrow W W$ $1.58 \pm 0.02 \mathrm{pb}$, single top ( $s$-channel) $0.88 \pm 0.05 \mathrm{pb}$, single top ( $t$-channel) $1.98 \pm 0.08 \mathrm{pb}$. Feynman diagrams for $s$ and $t$-channel single top production are shown in Figure 2.5.

The samples used to evaluate Monte Carlo acceptances and efficiencies are the same as those used for our pseudo-experiments. For the electroweak samples we use PYTHIA as an event generator and for parton showering, while for the single top case we use MadEvent + PYTHIA. In addition, the $t$-channel case involves a combination of LO and NLO samples. 
For pseudo-experiments we apply our entire set of event selection requirements to these samples. The number of expected events is determined by multiplying the theoretical cross section, the measured integrated luminosity, the pre-tag efficiency, and the event tagging efficiency. The event tagging efficiency is determined in the same way as for the $W+$ heavy flavor background, described above. The pre-tag efficiency is measured in Monte Carlo, and corrected for differences between data and Monte Carlo in lepton ID efficiencies, trigger efficiencies, and in the efficiency of events to be within the $60.0 \mathrm{~cm}$ fiducial region of the CDF detector. The lepton ID scale factors, trigger efficiencies, and $z_{0}$ efficiency are described elsewhere $[61,62,63]$.

\subsubsection{Non- $W$ QCD}

QCD multi-jet events can mimic our $t \bar{t}$ event signature in a variety of difficult to simulate ways. The necessary lepton can be a fake lepton from a jet, or a real lepton from a semi-leptonic hadron decay. Similarly, the $\mathbb{E}_{T}$ can be from a combination of jet mismeasurements or from a neutrino from a semi-leptonic decay. And, the $b$ tag can also be real or fake. Since the fake lepton and $\mathbb{E}_{T}$ processes are difficult to simulate and the heavy flavor content of QCD samples is not well known, these events are difficult to simulate and thus they, and the estimate of their number, are taken from data. We consider the lepton isolation and $\mathbb{E}_{T}$ sidebands to make the estimate, dividing the events from data into four regions:

- Region A: isolation $(I)>0.2$ and $\mathbb{E}_{T}<15 \mathrm{GeV}$

- Region B: $I<0.1$ and $\mathbb{E}_{T}<15 \mathrm{GeV}$

- Region C: $I>0.2$ and $E_{T}>20 \mathrm{GeV}$

- Region D (signal region): $I<0.1$ and $\mathbb{E}_{T}>20 \mathrm{GeV}$

We assume that, for the QCD events of interest, lepton isolation and $\notin_{T}$ are uncorrelated quantities. So, we can assume that the ratio of low isolation to high isolation QCD events at low $\mathbb{E}_{T}$ is the same as that at high $\mathbb{E}_{T}$ and so we can calculate the number of expected QCD events in the signal region as:

$$
N_{D}^{Q C D}=\frac{N_{B} N_{C}}{N_{A}}
$$


This equation is used to determine the number of pre-tag QCD events expected, and the number of tagged QCD events is computed as the weighted average of two estimates. The first estimate (the "tag method") uses Equation 4.9 directly with tagged events, but suffers from low statistics. The second estimate (the "pre-tag method") assumes that the tag rate is independent of $E_{T}$ and measures the tagging rate, in data, from Region B. This tagging rate is then applied to the pre-tag estimate from Equation 4.9.

Our events for pseudo-experiments similarly come from data. Specifically, we use events from Region C, with high $\mathbb{E}_{T}$ but also high isolation. Further details of the event selection are described elsewhere [64]. In order to ensure adequate statistics, or at least come as close as possible, we relax our $b$ tagging requirement when extracting QCD events for our pseudo-experiments. We consider the limited number of QCD events available as a systematic error, described later. We also consider the assumptions implicit in our modelling of background samples with a generous systematic error for background modelling, also described later. Neither of these sources of systematic error makes a significant contribution to the total systematic error.

\subsubsection{Systematic Error on Background Estimates}

Systematic uncertainties on the background estimates have been considered and are included in the estimates as shown in Table 4.3. The systematic uncertainties for each background are summarized in Table 4.4. The electroweak and single top estimates, since they are calculated using the integrated luminosity, and so are affected by the intrinsic resolution of the Cerenkov counters and by the uncertainty in the total $p \bar{p}$ cross section. The $W+$ heavy flavor estimate is affected by the details of the jet-parton matching prescription, by uncertainties in the modelling of initial and final-state radiation, and by other effects. The $W+$ light flavor (mistag) estimate is affected by uncertainties in the mistag matrix and in the calculation of the asymmetry factor. The error in the non- $W$ QCD estimate is evaluated by varying the size of the sideband regions, and by considering the error on the tag rate as estimated from Region B. Further details are available elsewhere [60]. 


\begin{tabular}{|c|c|c|}
\hline \multicolumn{2}{|l|}{ Background Level Fractional Uncertainties } \\
\hline EW + Single Top & $11 \%$ & $\begin{array}{c}\text { Lepton ID + modelling }(6.7 \%), b \text { tag SF }(6.6 \%), \\
\text { JES }(3.9 \%), \int L d t(6.0 \%)\end{array}$ \\
\hline W + HF & $26 \%$ & $\begin{array}{c}\text { Matching }(15 \%), \text { ISR } / \text { FSR }(10 \%), m_{Q}(6 \%), \\
\text { JES }(5 \%), \text { PDF }(5 \%), Q^{2} \text { scale }(4 \%), b \text { tag SF }(6.6 \%)\end{array}$ \\
\hline Mistags & $23 \%$ & Mistag matrix (8\%), light-flavor asymmetry $(22 \%)$ \\
\hline Non-W, Tag Method & $25 \%$ & Vary sidebands (25\%) \\
Non-W, pre-tag Method & $33 \%$ & Vary sidebands $(25 \%)$, tag rate $(20 \%)$ \\
\hline
\end{tabular}

Table 4.4: Systematic uncertainties on background estimates. 


\section{Chapter 5}

\section{General Analysis Method and Likelihood Formalism}

To extract the top mass from a sample of events in data or Monte Carlo simulation, we use the method of maximum likelihood [17] with a probability constructed from $t \bar{t}$ and background probabilities calculated using Standard Model matrix elements. Generally speaking, we make the hypothesis that our $N$ events observed in data, each with a set of measured properties $\left\{x_{i}\right\}$, come from a probability density function $f(x ; \theta)$ where $\theta$ is the set of unknown parameters that we wish to estimate. In our case, we will be especially interested in $m_{t}$. In general, the likelihood function is then the product of the individual event probabilities

$$
L(\theta)=\prod_{i=1}^{N} f\left(x_{i}, \theta\right)
$$

and our estimated value of theta is that which maximizes $L$ for the $N$ events we observe in data. Frequently, it's convenient to minimize - $\ln L$ instead of maximizing $L$. And then, to determine the $s$-standard deviation errors we form the contour

$$
-\ln L(\theta)=-\ln L_{\max }+\frac{s^{2}}{2}
$$

and the corresponding values of $L$ are the $s$-standard deviation errors around our best fit value of $\theta$.

For our measurement we are interested in the probability of observing events in our detector as a function of $m_{t}, \bar{P}\left(x ; m_{t}\right)$. Here $x$ are measured detector quantities such as jet and lepton energies and angles (rather than the parton quantities contained in the 
production and decay matrix elements) and the bar indicates that this is the probability to actually observe events, rather than to merely produce them. In general we write this probability as the sum of all possible event production processes, each of which might depend on $m_{t}$. These processes could include multiple processes for actually producing top quarks, such as $q \bar{q}, g g$, and single-top production - all of which naturally depend on $m_{t}$. They also include background processes without actual top quarks - these of course are independent of $m_{t}$. So, we have

$$
\bar{P}\left(x ; m_{t}\right)=\sum_{j=1}^{k} c_{j} \overline{P_{j}}(x)
$$

where $c_{j}$ is the fraction of the overall sample that comes from the process described by probability $\overline{P_{j}}$.

For this measurement, we consider two probabilities. The first, $\bar{P}_{t \bar{t}}\left(x ; m_{t}\right)$, is the probability of observing a set of detector quantities, $x$, because of $t \bar{t}$ production via $q \bar{q}$ annihilation. The other, $\bar{P}_{B a c k}(x)$, is the probability of observing $x$ from a single background process. We will discuss background probabilities based on the ALPGEN[48] WQ $\bar{Q}$ calculation and the VECBOS[53] $W+4 p$ calculation. For our measurement we use the ALPGEN-based probability while the VECBOS-based probability is an important cross check.

The division of processes between the background and signal categories is, to some extent, just a matter of nomenclature. We use only one $m_{t}$ dependent probability, that for $q \bar{q}$ production and decay, while not including a probability for $g g$ production. Our analysis is calibrated with simulated $t \bar{t}$ samples that do include $g g$ production and this simplified treatment of $g g$ events is treated as a systematic error. We use only a single background probability, but calibrate our analysis with simulated events containing the expected types and combinations of background events. These background events contain single-top events which are also sensitive to $m_{t}$. The uncertainties on the number and modelling of background events are considered as systematic errors.

Assuming that both the $t \bar{t}$ and background probabilities are normalized (we will discuss the signal and background normalizations when describing their respective probabilities), we have

$$
\bar{P}\left(x ; c_{1}, m_{t}\right)=c_{1} \bar{P}_{t \bar{t}}\left(x ; m_{t}\right)+\left(1-c_{1}\right) \bar{P}_{\text {Back }}(x) .
$$

So far we have been discussing the probability of observing a set of detector quan- 
tities $x, \bar{P}\left(x ; m_{t}\right)$, but it is difficult to write down an analytic expression for this. Instead, we relate the probability to observe $x, \bar{P}\left(x ; m_{t}\right)$, to the probability to produce $x, P\left(x ; m_{t}\right)$ :

$$
\bar{P}\left(x ; m_{t}\right) \propto A c c(x) P\left(x ; m_{t}\right)
$$

where $\operatorname{Acc}(x)$ is the acceptance of our detector and includes such quantities as the geometrical acceptance, event selection criteria like jet and lepton energy requirements, etc.

Keeping the individual $\bar{P}$ 's normalized we have

$$
\bar{P}_{t \bar{t}}\left(x ; m_{t}\right)=\frac{A c c(x) P_{t \bar{t}}\left(x ; m_{t}\right)}{\int A c c(x) P_{t \bar{t}}\left(x ; m_{t}\right) d x}=\frac{A c c(x) P_{t \bar{t}}\left(x ; m_{t}\right)}{\langle A c c(x)\rangle_{t \bar{t}}\left(m_{t}\right)}
$$

and

$$
\bar{P}_{B a c k}=\frac{A c c(x) P_{B a c k}(x)}{\int A c c(x) P_{B a c k}(x) d x}=\frac{A c c(x) P_{B a c k}(x)}{\langle A c c(x)\rangle_{B a c k}} .
$$

Here $\langle A c c(x)\rangle_{t \bar{t}}\left(m_{t}\right)$ is the average acceptance, averaged over all phase space, for events produced according to $P_{t \bar{t}}$ and $\langle A c c(x)\rangle_{B a c k}$ for events produced according to $P_{B a c k}$. Note that the average acceptance for the $t \bar{t}$ process depends on $m_{t}$. For example, we expect a larger fraction of events to pass our jet and lepton energy requirements when $m_{t}$ is large. The average background acceptance does not depend on $m_{t}$ unless $P_{\text {Back }}$ depends on $m_{t}$, and our $P_{\text {Back }}$ does not.

Since the common $A c c(x)$ term, in the numerator, does not depend on $m_{t}$, we can remove it from the likelihood - it will not affect the minimization. So, we have

$$
L\left(m_{t}\right)=\prod_{i=1}^{N} c_{1} \frac{P_{t \bar{t}}\left(x_{i} ; m_{t}\right)}{\langle A c c(x)\rangle_{t \bar{t}}\left(m_{t}\right)}+\left(1-c_{1}\right) \frac{P_{B a c k}\left(x_{i}\right)}{\langle A c c(x)\rangle_{B a c k}} .
$$

When we perform our likelihood fit for a particular collection of events, we will find the value of $m_{t}$ that minimizes $-\ln L$ (i.e. that maximizes $L\left(m_{t}\right)$ ). For the $b$-tagged Lepton + Jets sample the signal fraction, $c_{1}$, is well known. In general the requirement of a $b$ tag is important because it reduces background contamination. Because of this $t \bar{t}$ cross section analyses prefer the $b$-tagged sample and have studied its composition in detail. This precise knowledge of $c_{1}$ is a helpful feature for our analysis.

The $t \bar{t}$ acceptance is calculated by considering the fraction of fully simulated HERWIG $t \bar{t}$ events that pass our event selection requirements as described in Section 4.6. This fraction is considered as a function of $m_{t}$ and is shown in Figure 5.1. This first case is actually the acceptance for our baseline event selection, which we hold in common with other $t \bar{t}$ analyses. We must also account for the one event selection requirement unique to 


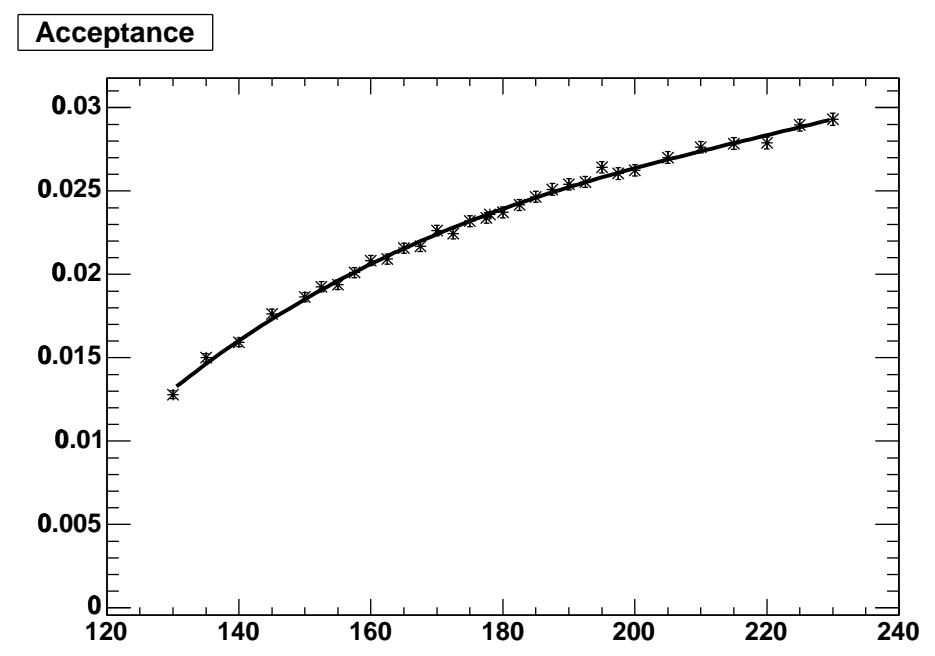

Figure 5.1: Signal $(t \bar{t})$ acceptance for baseline event selection, evaluated using fully simulated HERWIG $t \bar{t}$ events. Fractional Acceptance vs. $m_{t}\left(\mathrm{GeV} / c^{2}\right)$.

our analysis. This cut removes events with very low values of $P_{t \bar{t}}$ some of which exhibit a certain pathological behavior when processed by our $t \bar{t}$ signal probability. We will describe this $P_{t \bar{t}}$ cut in detail once we have developed the signal probability. The acceptance due to this additional cut is shown in Figure 5.2 while the overall signal acceptance, the product of these two pieces, is shown in Figure 5.3. The shape of this additional acceptance is not dramatic, ranging from almost $100 \%$ at low mass, to a few percent in the expected $m_{t}$ region, to as much as $10-12 \%$ for very lage $m_{t}$.

The background acceptance will be described in detail later, along with the background probability. 


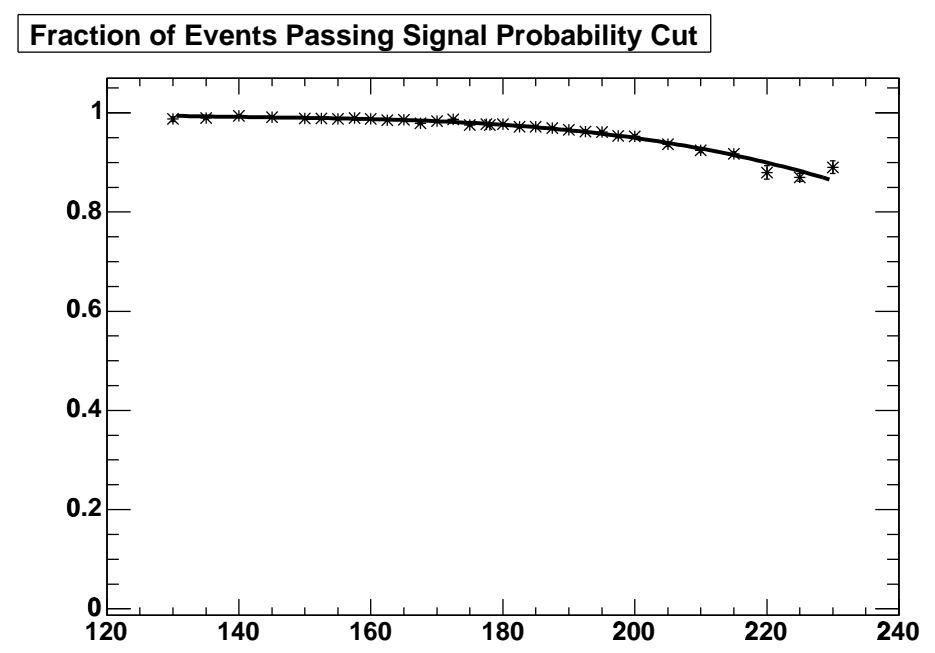

Figure 5.2: Signal $(t \bar{t})$ acceptance for $P_{t \bar{t}}$, evaluated using fully simulated HERWIG $t \bar{t}$ events. That is, the fraction of events, after regular event selection, that pass the additional $P_{t \bar{t}}$ cut. The shape of this additional acceptance is not dramatic when compared to the acceptance from the baseline event selection (see Figure 5.1), but it is also included in the likelihood. Fractional Acceptance vs $m_{t}\left(\mathrm{GeV} / c^{2}\right)$.

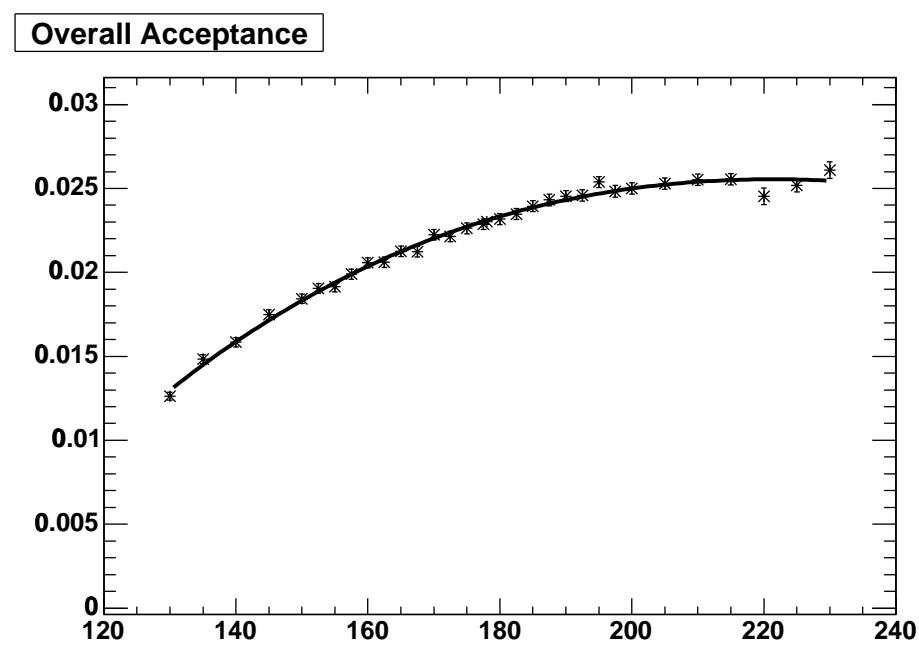

Figure 5.3: Signal $(t \bar{t})$ acceptance for overall event selection, the product of Figures 5.1 and 5.2. This is the acceptance used in Equation 5.8. Fractional Acceptance vs. $m_{t}$ $\left(\mathrm{GeV} / c^{2}\right)$. 


\section{Chapter 6}

\section{Transfer Functions}

Transfer functions map measured parton level quantities, $\vec{y}$, to reconstruction level quantities, $\vec{x}$. For example, a transfer function can include the probability of a particular parton energy resulting in a reconstructed calorimeter jet of a certain energy. We generally assume leptons and jet angles are well measured, such that the transfer function contains delta-functions for these parameters, leaving only the mapping between jet and parton energy. The full transfer function in this case is given by

$$
W(\vec{x}, \vec{y})=\delta^{3}\left(p_{\nu}^{y}-p_{\nu}^{x}\right) \delta^{3}\left(p_{l}^{y}-p_{l}^{x}\right) \prod_{i=1}^{4} W_{j e t}\left(E_{i}^{y}, E_{i}^{x}\right) \prod_{j=1}^{4} \frac{1}{\left(p_{j}^{x}\right)^{2}} \delta^{2}\left(\Omega_{j}^{y}-\Omega_{j}^{x}\right) .
$$

We designate the jet/parton energy mapping, $W_{\text {jet }}\left(E_{i}^{x}, E_{i}^{y}\right)$, the jet energy transfer function, but we will call it simply the transfer function and may often omit the subscript. This chapter is devoted to the parameterization of this term. The specific implementation of the transfer functions has been revisited several times in the course of our analysis [65]. More details on the particular implementation used for the $318 \mathrm{pb}^{-1}$ analysis can be found in reference [66].

\subsection{Jet Energy Transfer Functions}

Jet energies are measured as energy depositions in the calorimeter and reconstructed using a jet clustering algorithm. We have described jet energy measurements briefly in Section 4.4.2 and the corrections used in common by many analyses at CDF are described in detail elsewhere [59]. But, by way of introduction, we outline the approach 
again here. Since the calorimeter is not perfect, many corrections are applied to the measured energies in order to determine the original energy of the group of particles incident on the calorimeter. That is, to correct the calorimeter jet energy back to the energy of the particle jet. Non-instrumented regions and the non-linear calorimeter response are taken into account using generic corrections [67].

Unlike some other physics analysis, the measurement of top quark properties generally relies on a knowledge of the parton level energies. The usual strategy for the measurement of the top mass is to estimate the parton level four vectors of the partons, lepton, and neutrino from $t \bar{t}$ decay. Knowing these four vectors we could, in principle, simply add them back up the decay chain and take invariant masses to find $m_{t}$.

These parton level four vectors are usually obtained by applying generic out-of-cone jet corrections (OOC) [68] or top specific corrections (TS) [69] after the generic ${ }^{1}$ calorimeter corrections are applied to the jet energies. The top specific corrections are extracted from simulated $t \bar{t}$ events and so have, for example, the same jet $p_{T}$ spectrum as we expect from $t \bar{t}$ events in data. There are also separate top specific corrections for light jets and $b$ jets, whose calorimeter response we expect to be significantly different because of, for example, semi-leptonic $b$ decays.

These OOC and TS corrections use the most probable value or the mean of the difference between jet energies and parton energies $\left(\delta_{E}\right)$ and apply a Gaussian assumption. In reality the $\delta_{E}$ distributions are not symmetrical, and the $b$ jet distributions very noticeably not so. So, the Gaussian assumption can be significantly limiting. Our transfer function accounts for this by describing the full shape of the distribution as a probability density. Matrix element analyses take advantage of the additional information in their integration over parton energy distributions.

The transfer function, $W_{\text {jet }}\left(E_{\text {jet }}, E_{\text {parton }}\right)$, is defined as the probability of measuring a jet with $E_{j e t}$ given a parton with fixed value of $E_{\text {parton }}$. They are similar to the ones obtained for the generic OOC corrections from di-jet events [68] and are inspired by the transfer functions used in the D0 matrix element analyses [23]. We parameterize the transfer function as the sum of two Gaussians:

$$
W_{\text {jet }}\left(E_{\text {jet }}, E_{\text {parton }}\right)=\frac{1}{\sqrt{2 \pi}\left(p_{2}+p_{3} p_{5}\right)}\left[\exp \frac{-\left(\delta-p_{1}\right)^{2}}{2 p_{2}}+p_{3} \exp \frac{-\left(\delta-p_{4}\right)^{2}}{2 p_{5}}\right]
$$

\footnotetext{
${ }^{1}$ We call these corrections generic because they are used by a wide range of analyses and are not meant to be specific to a particular hard scattering physics process.
} 
where $\delta \equiv E_{\text {parton }}-E_{\text {jet }}$. Conceptually, one Gaussian function describes the symmetric peak while the other accommodates the asymmetric tails of the $\delta \equiv E_{\text {parton }}-E_{\text {jet }}$ distribution. We normalize Equation 6.2 in $E_{\text {jet }}$ such that it describes the probability of observing a jet with $E_{\text {jet }}$ produced by a parton with $E_{\text {parton }}$ :

$$
\int W_{j e t}\left(E_{j e t}, E_{\text {parton }}\right) d E_{j e t}=1 .
$$

We allow each of the parameters to depend linearly with parton energy ${ }^{2}$. We therefore parameterize this dependence with $p_{i}=a_{i}+b_{i} E_{\text {parton }}$, resulting in a total of 10 parameters describing the transfer function.

The parameterization of Equation 6.2 is perhaps semi-empirical, but certainly not unique. Other parameterization, such as a single Gaussian or a Pearson 4 function, have been considered but found to offer no significant improvement.

The transfer function parameters are extracted from fully simulated $t \bar{t}$ Monte Carlo events. We begin by applying the full offline reconstruction and event selection and then match reconstructed jets with partons. As we have stated, we expect the $\delta_{E}$ distributions to differ for $b$ quarks and light quarks and therefore, obtain 2 different transfer functions. We use pairs of matched jets and partons requiring $\Delta R \equiv \sqrt{\Delta \phi^{2}+\Delta \eta^{2}}<0.15$, and that there are no other jets or partons within $\Delta R<0.6$. Different such isolation requirements have been explored.

After event selection is applied, we do not require that all of the jet-parton pairs in an event pass our matching requirement - we extract parameters from any pair passing the requirement. To determine the parameters in Eq. 6.2 we use an unbinned likelihood fit:

$$
-\ln \mathcal{L}=-\sum_{i=1}^{N} \ln W_{\text {jet }}\left(E_{\text {jet }}, E_{\text {parton }}\right)
$$

Our default transfer function is derived from HERWIG $t \bar{t} M_{t o p}=178 \mathrm{GeV} / c^{2}$ The parameters for this default transfer function are shown in Table 6.1.

\subsection{Tests and Cross-Checks}

We check the quality of the transfer function parameterization by comparing reconstruction level distributions with predictions obtained using the transfer function and

\footnotetext{
${ }^{2}$ A quadratic dependence was also considered but found not to offer any significant improvement.
} 
Table 6.1: The parameters for our default transfer function, with the parameterization of Equation 6.2. The default transfer function is extracted from HERWIG $t \bar{t} M_{t o p}=178$ $\mathrm{GeV} / c^{2}$ Monte Carlo.

\begin{tabular}{|c|c|c|c|c|}
\hline \hline & \multicolumn{2}{|c|}{ light quark jets } & \multicolumn{2}{c|}{ b quark jets } \\
$p_{i}=a_{i}+b_{i} E_{\text {parton }}$ & $a_{i}$ & $b_{i}$ & $a_{i}$ & $b_{i}$ \\
\hline$p_{1}(\mathrm{GeV})$ & 1.09923 & -0.0522277 & -1.59438 & -0.0473066 \\
$p_{2}(\mathrm{GeV})$ & 2.02192 & 0.103122 & 2.77686 & 0.0737285 \\
$p_{3}$ & 0.0506705 & $5.69989 \mathrm{e}-12$ & 0.413792 & $1.78746 \mathrm{e}-12$ \\
$p_{4}(\mathrm{GeV})$ & 25.0381 & -0.531444 & 5.57249 & -0.261912 \\
$p_{5}(\mathrm{GeV})$ & 10.9866 & 0.114647 & 0.991688 & 0.197914 \\
\hline \hline
\end{tabular}

parton level quantities in Monte Carlo. Two types of tests are done. The first compares simple quantities like jet energies and the second compares two-jet and three-jet invariant masses. So, the first is a low-level test while the second relates more directly to the variables used in the $t \bar{t}$ probability. Both of these types of tests are, in a sense, preliminary. They aim only to characterize, and imperfectly, the performance of the transfer functions. Ultimately the most important tests are of the transfer function in the context of the overall analysis technique. These tests will come in Chapters 7 and 9.

\subsubsection{One-Dimensional Checks}

Our one-dimensional checks compare one-dimensional slices of the $E_{\text {jet }}, E_{\text {parton }}$ space used to extract the transfer functions.

The jet and parton level quantities are known in Monte Carlo, and we compare them with an analytical expression involving parton energies and the transfer function. To calculate the parton distributions, $H$, we convolute the transfer function, $W_{j e t}$, with the parton energy spectrum, $n\left(E_{\text {parton }}\right)$. That is,

$$
\begin{aligned}
H\left(E_{j e t}\right) & =\int_{E_{\text {parton }}^{1}}^{E_{\text {parton }}^{2}} d E_{\text {parton }} n\left(E_{\text {parton }}\right) W_{\text {jet }}\left(E_{\text {jet }}, E_{\text {parton }}\right) \\
H\left(E_{\text {parton }}\right) & =\int_{E_{\text {jet }}^{1}}^{E_{\text {jet }}^{2}} d E_{j e t} n\left(E_{\text {parton }}\right) W_{\text {jet }}\left(E_{\text {jet }}, E_{\text {parton }}\right) \\
H\left(\delta_{E}\right) & =\int_{E_{\text {parton }}^{1}}^{E_{\text {parton }}^{2}} n\left(E_{\text {parton }}\right) W_{\text {jet }}\left(E_{\text {parton }}-\delta_{E}, E_{\text {parton }}\right)
\end{aligned}
$$

where the integral over $d E_{i}$ is over the range of the bins in the histogrammed jet distribu- 
tions. We fit the parton energy distribution in two different ways for the light and b-quarks:

$$
\begin{aligned}
n\left(E_{\text {parton }}^{\text {light quarks }}\right) & =c_{1} \exp \left(c_{2}-E_{\text {parton }}\right)^{2} / 2 c_{3}^{2}+c_{4} \exp \left(c_{5}-E_{\text {parton }}\right)^{2} / 2 c_{6}^{2} \\
n\left(E_{\text {parton }}^{\text {b-quarks }}\right) & =c_{1}\left(E_{\text {parton }}-c_{2}\right)^{c_{3}} \exp \left[-c_{4}\left(E_{\text {parton }}-c_{2}\right)\right]
\end{aligned}
$$

Table 6.2 lists the parameters derived from Herwig $M_{t o p}=178 \mathrm{GeV} t \bar{t}$ Monte Carlo and Figure 6.1 and 6.2 show the fits to the light and $b$-quark parton energy distributions.

Table 6.2: Parton energy distribution parameters corresponding to Eq. 6.6.

\begin{tabular}{|c|c|c|c|c|c|c|}
\hline & $c_{1}$ & $c_{2}$ & $c_{3}$ & $c_{4}$ & $c_{5}$ & $c_{6}$ \\
\hline light quarks & 242.08 & -676.23 & 184.77 & -0.30182 & -3.8753 & 25.611 \\
\hline b quarks & 0.22263 & 38.792 & 1.21023 & 0.032703 & 0.016693 & $\mathrm{n} / \mathrm{a}$ \\
\hline
\end{tabular}

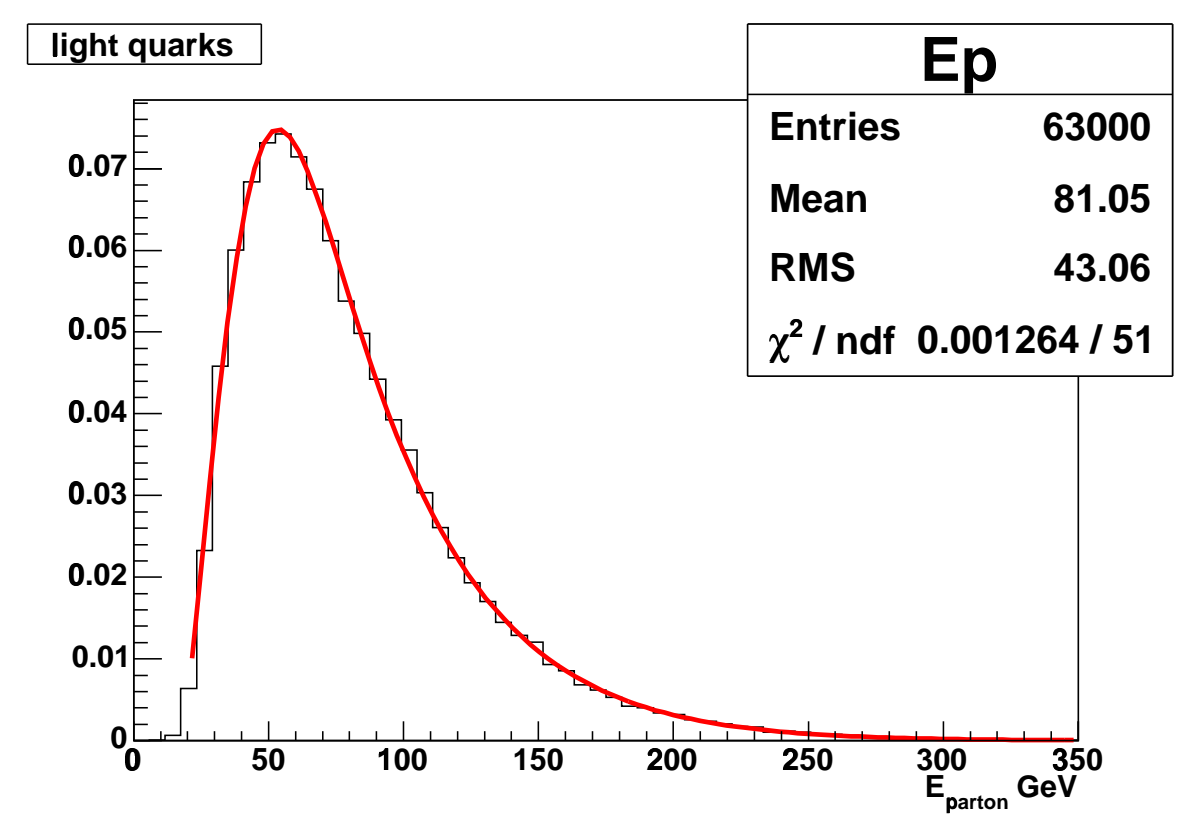

Figure 6.1: Distribution of light quark energies from Herwig $M_{t o p}=178 \mathrm{GeV} t \bar{t}$ Monte Carlo. The line is a fit to the histogram using Eq. 6.6.

The comparison of simulated events to predictions from parton-level events convoluted with the transfer function is show for $\delta_{E} \equiv E_{\text {parton }}-E_{\text {jet }}$ in Figure 6.3 for light quarks and Figure 6.4 for $b$ quarks. Here the asymmetric nature of the jet response curves is evident, and especially so for $b$ quarks at high energy. Fairly good agreement is observed between Monte Carlo and predictions, in both the central measures and the shape of the 


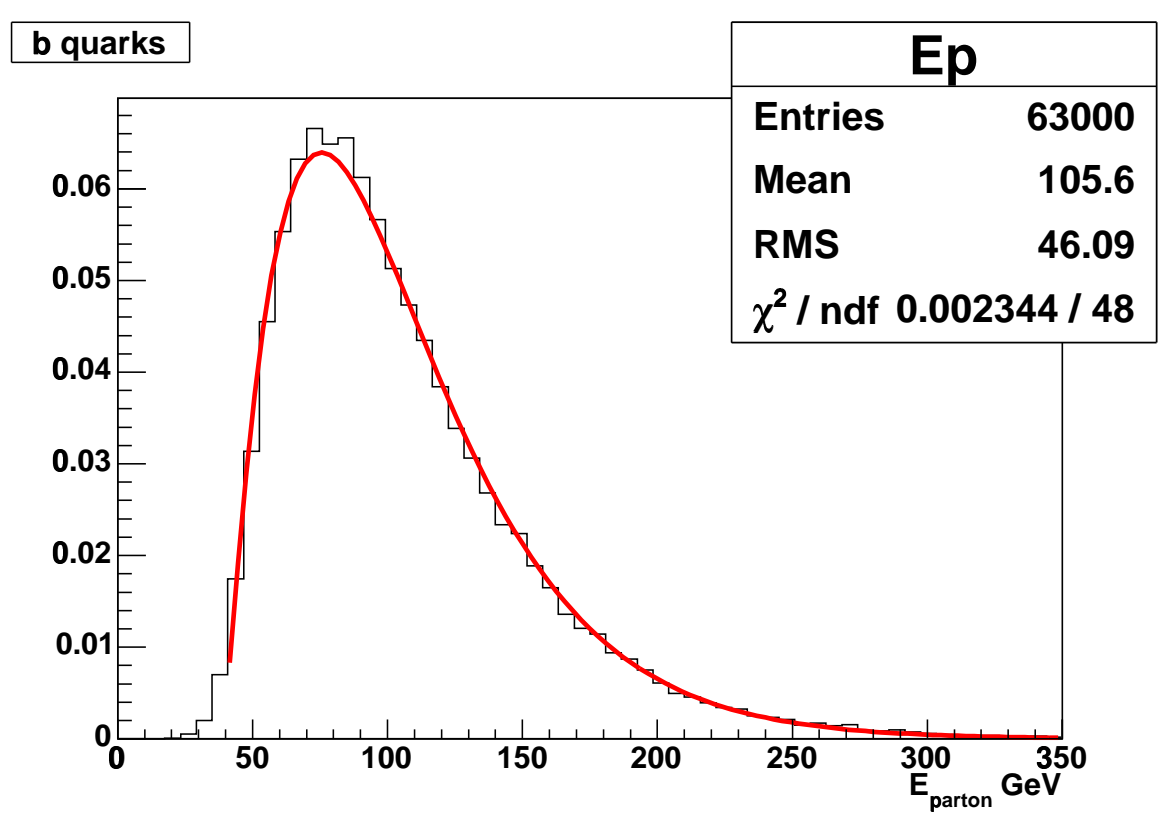

Figure 6.2: Distribution of $b$ quark energies from Herwig $M_{t o p}=178 \mathrm{GeV} t \bar{t}$ Monte Carlo. The line is a fit to the histogram using Eq. 6.6.

distributions. We do not attempt to quantify the level of agreement between the histogram and curve, but use these plots as an indication that our parameters and parameterization are reasonable. Quantitative studies are performed with the invariant mass tests. Comparisons for the other predictions of Equation 6.6 can be found elsewhere [66].

\subsubsection{Two-jet and Three-jet Invariant Masses}

A higher-level test of the transfer function is made by comparing the two-jet and three-jet invariant masses from the Monte Carlo to the predictions obtained from the transfer function. We follow the technique used in reference [70].

In general, given an acceptance function, $\operatorname{Acc}(\vec{x})$, and a normalized probability density, $P(\vec{x})$, we can calculate the number of observed events with:

$$
N=\int \operatorname{Acc}(\vec{x}) P(\vec{x}) d \vec{x}
$$

To determine the two-jet or three-jet invariant mass we calculate $d N / d m_{x}$, changing vari- 


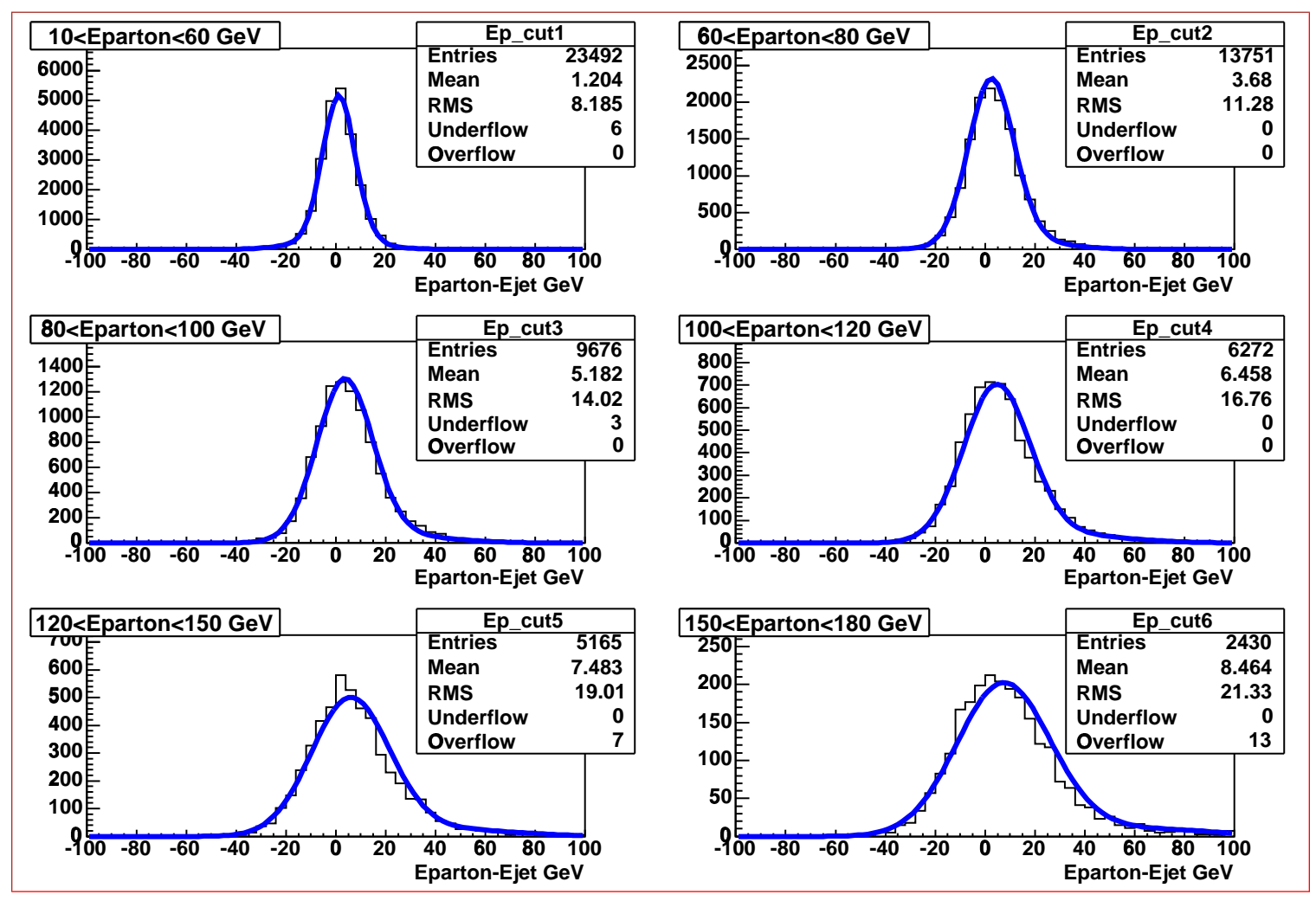

Figure 6.3: Comparison of light jet $E_{\text {parton }}-E_{\text {jet }}$ distribution from Monte Carlo, in bins of $E_{\text {parton }}$, with the prediction from parton-level events convoluted with the transfer function.

ables from $\vec{x}=\left(x_{1}, x_{2}, \ldots, x_{n}\right)$ to $\left(m_{x}, x_{2}, \ldots, x_{n}\right)$ :

$$
\begin{aligned}
N & =\int \operatorname{Acc}(\vec{x}) P(\vec{x}) d \vec{x} \\
& =\int d m_{x} d x_{2} \ldots d x_{n} J\left(\frac{x_{1}, x_{2}, \ldots, x_{n}}{m_{x}, x_{2}, \ldots, x_{n}}\right) \operatorname{Acc}(\vec{x}) P(\vec{x})
\end{aligned}
$$

with the Jacobian specified by:

$$
J\left(\frac{m_{x}, x_{2}, \ldots, x_{n}}{x_{1}, x_{2}, \ldots, x_{n}}\right)=\left|\begin{array}{cccc}
\frac{\partial m_{x}}{\partial x_{1}} & \frac{\partial m_{x}}{\partial x_{2}} & \ldots & \frac{\partial m_{x}}{\partial x_{n}} \\
\frac{\partial x_{2}}{\partial x_{1}} & \frac{\partial x_{2}}{\partial x_{2}} & \ldots & \frac{\partial x_{2}}{\partial x_{n}} \\
\vdots & \vdots & \ldots & \vdots \\
\cdot & \cdot & \cdot \\
\frac{\partial x_{n}}{\partial x_{1}} & \frac{\partial x_{n}}{\partial x_{2}} & \ldots & \frac{\partial x_{n}}{\partial x_{n}}
\end{array}\right|
$$




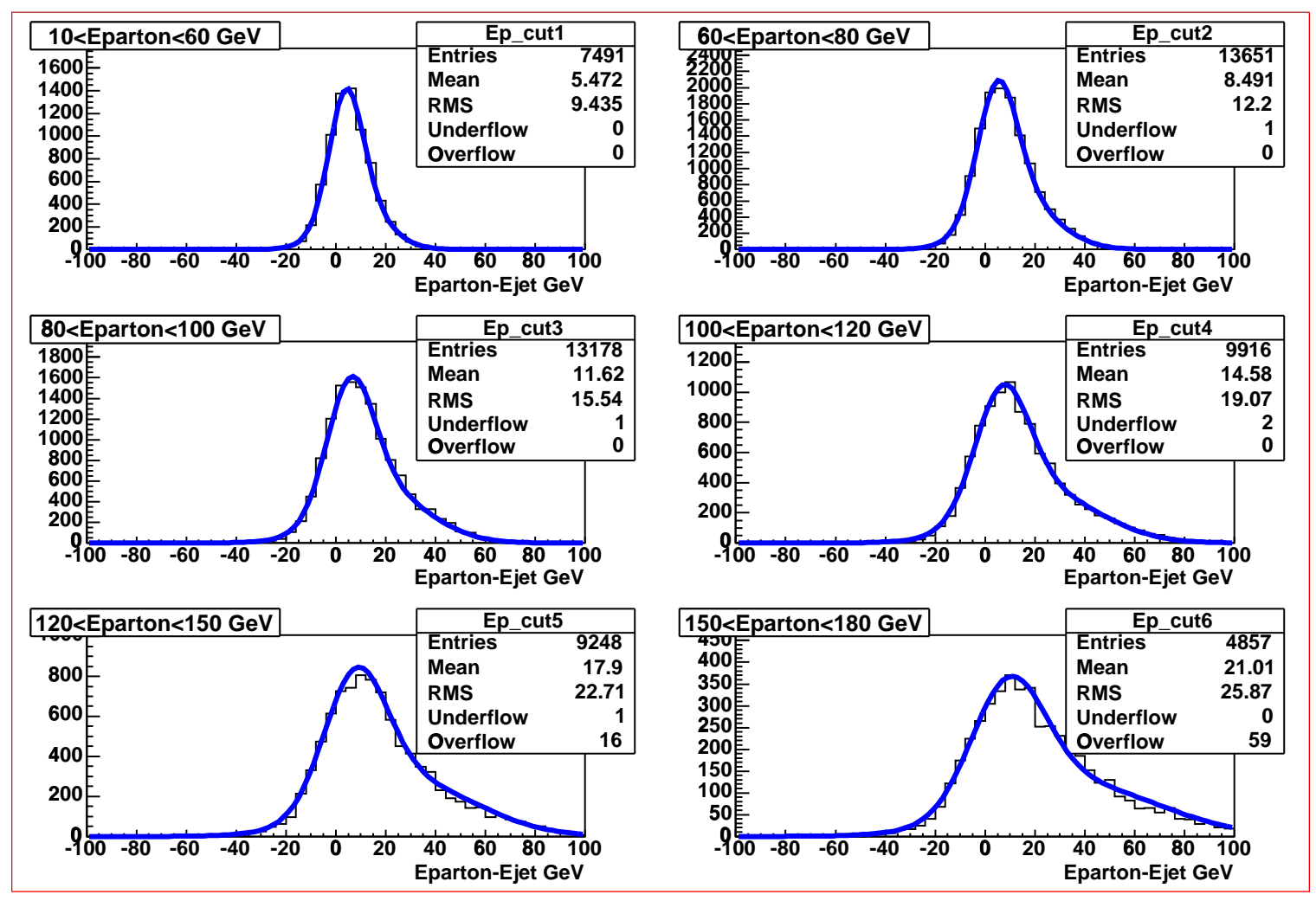

Figure 6.4: Comparison of $b$ jet $E_{\text {parton }}-E_{\text {jet }}$ distribution from Monte Carlo, in bins of $E_{\text {parton }}$, with the prediction from parton-level events convoluted with the transfer function. The asymmetric shape of the jet response is clear, especially for high energy $b$ jets. We do a fairly good job of modelling the shape of the response as well as the central measures.

The differential cross-section for $t \bar{t}$ events is given by

$$
P(\vec{x})=\int d^{n} \sigma(\vec{y}) d q_{1} d q_{2} f\left(q_{1}\right) f\left(q_{2}\right) W(\vec{x}, \vec{y})
$$

where $\vec{x}$ indicates reconstruction level quantities and $\vec{y}$ indicates parton level quantities. Summing over possible permutations of jet and parton combinations and integrating over the delta functions in $W(\vec{x}, \vec{y})$, results in

$$
\frac{d N}{d m_{x}}=\int d^{n} \sigma(\vec{y}) d q_{1} d q_{2} f\left(q_{1}\right) f\left(q_{2}\right) Q(\vec{y})
$$

where we define the integral over the remaining reconstruction level quantities - three of 
the jet energies - as

$$
Q(\vec{y}) \equiv \sum_{\text {perm. }} \int d x_{2} d x_{3} d x_{4} J\left(\frac{x_{1}, x_{2}, \ldots, x_{n}}{m_{x}, x_{2}, \ldots, x_{n}}\right) \operatorname{Acc}(\vec{x}) \prod_{i j e t=1}^{4} W_{j e t}\left(E_{i}^{x}, E_{i}^{y}\right)
$$

We integrate over the parton quantities with Monte Carlo, simply using the partonlevel events in our fully simulated Monte Carlo samples. Since we also have full reconstruction information for the parton events, we effectively integrate over the acceptance, $\operatorname{Acc}(\vec{x})$, in $Q(\vec{y})$ by requiring our partons to pass the usual event selection cuts at reconstruction level.

In the Monte Carlo integration of the invariant mass distribution, we numerically evaluate $Q(\vec{y})$ (not including acceptance) for each parton event.

To avoid edge effects in the integration, we set the cutoff on jet energy slightly lower in the MC than in the final analysis. Ignoring the mass of the W-daughters, we define the invariant masses as

$$
\begin{aligned}
m_{j j}^{2} & =E_{1} E_{2}\left(1-\cos \theta_{12}\right) \\
m_{j j j}^{2} & =m_{b}^{2}+2\left(E_{b} p_{1}-p_{b} p_{1} \cos \theta_{\mathrm{b} 1}\right)+2\left(\mathrm{E}_{\mathrm{b}} \mathrm{p}_{2}-\mathrm{p}_{\mathrm{b}} \mathrm{p}_{2} \cos \theta_{\mathrm{b} 2}\right) \\
& +2 p_{1} p_{2}\left(1-\cos \theta_{12}\right)
\end{aligned}
$$

The Jacobians are

$$
\begin{aligned}
J\left(\frac{E_{1} E_{2}}{m_{j j} E_{2}}\right) & =\frac{m_{j j}}{E_{2}\left(1-\cos \theta_{12}\right)} \\
J\left(\frac{E_{1} E_{2}}{m_{j j j} E_{2}}\right) & =\frac{m_{j j j}}{E_{b}-p_{b} \cos \theta_{\mathrm{b} 1}+\mathrm{E}_{2}\left(1-\cos \theta_{12}\right)}
\end{aligned}
$$

This results in a prediction of the invariant mass distribution based on the partons used in integration and the parameters of the transfer function.

We construct the invariant mass plots by comparing the predicted distribution with the distribution of the invariant masses of the reconstruction level jets. Since the normalization of the parton curve is not determined by our calculation, we normalize the parton curve to have the same integral as the area under the jet histogram. We estimate the relative shift between the parton curve and the jet histogram by calculating the $\chi^{2}$ between them for offsets of five bins in either direction. The minimum of the resulting curve is taken to be the shift and the error to be the width at which the value is one greater than that of the minimum. 

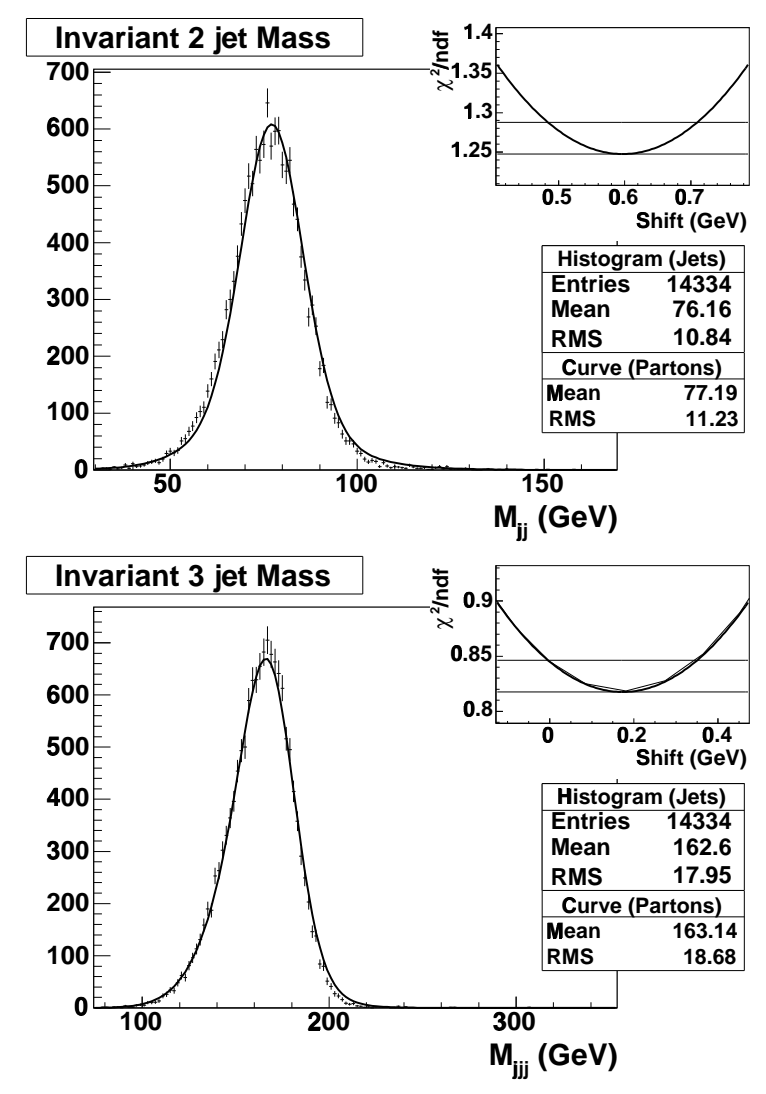

Figure 6.5: The curve was obtained using the default transfer function, and calculated using only the correct combination and using parton-level events from matched-to-jets partons. The invariant masses from the histogram were calculated using matched-to-partons jets. All samples are generated with $M_{t o p}=178 \mathrm{GeV} / c^{2}$. The $\chi^{2}$-fit range is $65-90 \mathrm{GeV} / c^{2}$ in $m_{j j}$ and $120-190 \mathrm{GeV} / c^{2}$ in $m_{j j j}$.

Figure 6.5 shows the two- and three-jet invariant mass comparisons for our default transfer function parameters with the correct jet-parton assignment. We use matched-tojets partons in the prediction curve and matched-to-partons jets in the reference histogram.

Note that the expected values of $m_{j j}$ and $m_{j j j}$ here are not $80 \mathrm{GeV} / c^{2}$ and 178 $\mathrm{GeV} / c^{2}$. We are attempting to reproduce the results of fully simulated events by convoluting parton level events with the transfer function. So, the goal here is to reproduce the jetlevel results. Our transfer functions connect partons to particle-level jets and so the $m_{j j}$ and $m_{j j j}$ that we expect are those of particle-level jets, and so lower than the nominal 80 $\mathrm{GeV} / c^{2}$ and $178 \mathrm{GeV} / c^{2}$.

Figure 6.6 shows the same comparison, but with all 12 possible permutations of jet 

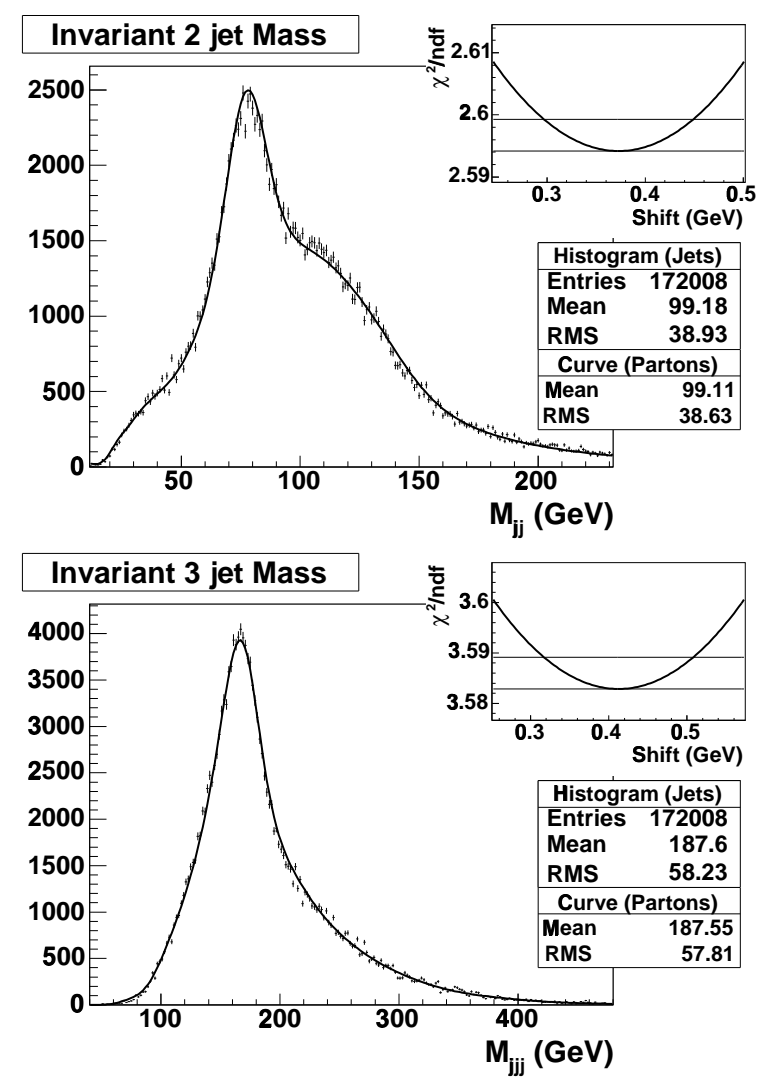

Figure 6.6: The curve is the prediction obtained using the default transfer function as in Fig. 6.5 but calculating all 12 combinations with matched-to-jets partons. The invariant masses from the histogram were calculated using matched-to-partons jets and using all the permutations among jets. All samples are generated with $M_{t o p}=178 \mathrm{GeV} / c^{2}$. The $\chi^{2}$-fit range $25-220 \mathrm{GeV} / c^{2}$ in $m_{j j}$ and $80-400 \mathrm{GeV} / c^{2}$ in $m_{j j j}$.

and parton pairing. Note that each event has 12 entries in each histogram, corresponding to the 12 possible parton-jet combinations..

Finally, in Figure 6.7 we relax the matching requirement on the jets and partons, allowing all events passing selection. The matching requirement selects an unnaturally clean set of events, usually events which at jet level look quite a lot like parton level. Many events with radiation, merged or split jets, or significant angular deviations between partons and jets are removed with the matching requirement. Of course we cannot make this requirement in data, so this selection is in this sense the most realistic. But, in our actual analysis we have the signal and background probabilities to help emphasize well measured $t \bar{t}$-like events and to emphasize the best combinations. In Figure 6.7 all combinations and 

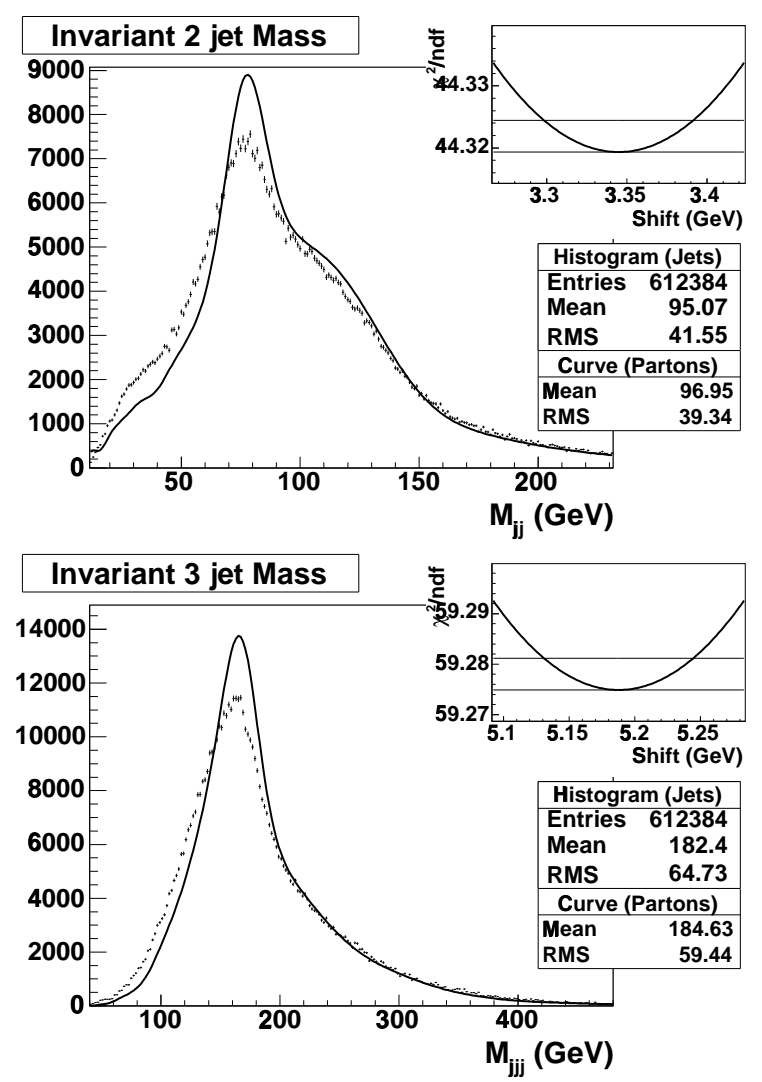

Figure 6.7: The curve is the prediction obtained using the default transfer function as in Fig. 6.5 but calculating all 12 combinations with matched-to-jets partons. The invariant masses from the histogram were calculated using all jets, without requiring matching to partons, and using all the permutations among jets. All samples are generated with $M_{\text {top }}=178$ $\mathrm{GeV} / c^{2}$. The $\chi^{2}$-fit range $25-220 \mathrm{GeV} / c^{2}$ in $m_{j j}$ and $80-400 \mathrm{GeV} / c^{2}$ in $m_{j j j}$.

all events contribute equally. So, the relatively poor agreement between prediction and full simulation shown here does not necessarily represent disaster. The most complete and important tests will come with our full analysis machinery, including the transfer function, in Chapters 7 and 9.

Table 6.3 summarizes the results of the invariant mass tests for our transfer function parameters derived from HERWIG, using HERWIG events in the Monte Carlo integration and reference histogram. We observe a shift of $0.6 \mathrm{GeV} / c^{2}$ in the two-jet mass and a shift consistent with zero in the three-jet mass for the correct jet/parton combination. When considering all possible jet/parton permutations, we see a $0.4 \mathrm{GeV} / c^{2}$ shift in both the two- and three-jet invariant masses in the matched case and significant biases in the 
Table 6.3: Summary of $m_{j j}$ and $m_{j j j}$ shifts in transfer function tests. The results are shown graphically in Figures 6.5, 6.6, and 6.7.

\begin{tabular}{|l|c|c|}
\hline \hline Sample & $m_{j j}$ & $m_{j j j}$ \\
\hline Matched Corr. Combo & $0.60 \pm 0.11$ & $0.17 \pm 0.18$ \\
Matched 12 Combos & $0.37 \pm 0.08$ & $0.41 \pm 0.10$ \\
Unmatched 12 Combos & $3.3 \pm 0.05$ & $5.2 \pm 0.06$ \\
\hline \hline
\end{tabular}

unmatched case.

This last results suggest that our transfer functions do not a very good job in the case of events where we are unable to match jets to partons. This is not really a surprise. The transfer functions map parton and jet energies only. So, for events where the parton angles do not correspond to the jet angles (e.g. unmatchable events) they are not expected to perform well. We would expect these effects to be present in the $m_{j j}$ and $m_{j j j}$ tests but not in the earlier one-dimensional tests.

\subsection{Systematic Studies}

We have conducted a number of additional tests of the quality of our particular transfer function fit and checks for systematic biases arising from the parameterization and assumptions made while extracting the transfer function parameters.

We explored the possible advantages of having $\eta$-dependent transfer functions. We divided the calorimeter into five bins in $\eta$ and fit a transfer function in each separately. No significant advantage was found. We also tested the $m_{t}$-dependence of transfer functions. We applied our default transfer function (extracted from $m_{t o p}=178 \mathrm{GeV} / c^{2}$ Monte Carlo) to samples with $m_{t}$ ranging from 130 to $210 \mathrm{GeV} / c^{2}$ and it worked well in each case (though showed some problems when applied to the extreme $230 \mathrm{GeV} / c^{2}$ sample). This is the behavior we expect, as we will describe further in Section 6.4. We considered differences between two popular comprehensive event generation codes, Pythia and Herwig. We extracted transfer functions from Herwig (our default) and applied them to Pythia, and vice versa. Differences were seen at the level of about $0.5 \mathrm{GeV} / c^{2}$ in $m_{j j}$ and $m_{j j j}$ tests. Many other studies were conducted, including the effects of under- or over-estimated radiation in the Monte Carlo samples, and the particular matching requirements imposed. These are documented in more detail elsewhere [66]. Other tests were conducted in an earlier version 
of our analysis [65]. These included other possible parameterizations of the transfer functions, and the possible effect of systematic differences in the opening angles of the parton pair from $W$ decay and the subsequent pair of jets.

In all cases those systematics that we expect to actually affect our final measured $m_{t}$ value, that is those that represent known or suspected differences between data and Monte Carlo, will be considered with the full analysis machinery in Chapter 11.

\subsection{Comparison of Transfer Functions in Different Analyses}

The top mass measurement at CDF using the Dynamical Likelihood Method (DLM) [27] also uses transfer functions that aim to relax the Gaussian assumption and model the shape of the jet response as well as the central measures. But, the DLM transfer functions, along with the traditional top-specific and generic jet corrections at CDF, operate in the opposite sense from the transfer functions documented in this thesis.

The DLM transfer functions and CDF generic jet corrections take a jet energy as input and return a parton energy. They ask questions such as, "Given a $50 \mathrm{GeV}$ jet, what is the probability that it came from a $40 \mathrm{GeV}$ parton?". The answer necessarily depends on how common a $40 \mathrm{GeV}$ parton is in a particular physical process relative to, say, a 50 $\mathrm{GeV}$ parton. This means that these corrections are necessarily dependent upon the parton $p_{t}$ spectrum of the particular process in question, and we expect them to vary from process to process as well as from $m_{t}$ to $m_{t}$.

The transfer functions we have described in this thesis instead take a parton energy as input and return a jet energy. They ask questions such as, "Given a $40 \mathrm{GeV}$ parton, what is the probability that it will result in a $50 \mathrm{GeV}$ jet?". The answer of course depends on the flavor of the parton. But, a particular $40 \mathrm{GeV} b$ parton fragments and hadronize into a particle jet (and then interacts in the calorimeter) in a manner relatively independent of the

details of the underlying hard scattering process. So, we expect our transfer functions to be relatively independent of process, and quite independent of the particular $m_{t}$ of the Monte Carlo sample from which they were extracted. As an extreme case, you might expect $t \bar{t}$ transfer functions to be applicable even to di-jet events. But, we do expect transfer functions to depend on parton flavor (gluon vs. light vs. $b$ ) and they will of course not be completely independent of the particle content and color flow of the rest of the event.

There are of course other differences between DLM transfer functions and those 
described in this thesis. There are other differences in assumptions made, and in details of the transfer function extraction and application. But, the one key philosophical difference we have described is especially worth noting. 


\section{Chapter 7}

\section{Signal Probability}

\subsection{Signal Probability in Analytic Form}

To use the likelihood from Equation 5.8 we will need an expression for $P_{t \bar{t}}\left(x ; m_{t}\right)$, the probability of producing a $t \bar{t}$ event with kinematics $x$, where $x$ are quantities measured by the CDF detector such as jet and lepton energies and angles. To construct this probability we would like to compare our measured event properties with our Standard Model knowledge of the physics of $t \bar{t}$ production and decay, as contained in the leading-order matrix element and differential cross section.

We can write this probability as

$$
P_{t \bar{t}}\left(x ; m_{t}\right)=\frac{1}{\sigma\left(m_{t}\right)} \int d \sigma(y) d q_{1} d q_{2} f\left(q_{1}\right) f\left(q_{2}\right) W(x ; y)
$$

where $x$ is the set of detector-level (e.g. jet) 4-vectors and $y$ is the set of parton-level 4 -vectors. $d \sigma(y)$ is the parton-level differential cross section. $W(x ; y)$ is the generalized transfer function of of Equation 6.1 giving the probability for an event to have detectorlevel quantities $x$ when parton-level quantities $y$ are produced. The two additional integrals, $d q_{1} f\left(q_{1}\right)$ and $d q_{2} f\left(q_{2}\right)$ enter because $d \sigma(y)$ will be the parton-level cross section, but we are considering the process $p \bar{p} \rightarrow t \bar{t}$. So, $f\left(q_{i}\right)$ are the parton distributions with $f\left(q_{i}\right) d q_{i}$ the probability that parton $i$ carries longitudinal momentum $q_{i}$, a fraction of the $980 \mathrm{GeV} / c$

proton momentum. $\sigma\left(m_{t}\right)$, the total cross section, in the denominator normalizes $P_{t \bar{t}}\left(x ; m_{t}\right)$ so that it is a properly normalized probability. Note that $\sigma\left(m_{t}\right)$ depends on the top mass. 
Following [17], we write

$$
d \sigma=\frac{(2 \pi)^{4}|M|^{2}}{4 \sqrt{\left(q_{1} \cdot q_{2}\right)^{2}-m_{1}^{2} m_{2}^{2}}} d \Phi_{n} .
$$

Here $q_{1}$ and $q_{2}$ are the four-vectors of the incoming $q$ and $\bar{q}$ with masses $m_{1}$ and $m_{2}, M$ is the matrix element for the process of interest, and $d \Phi_{n}$ is an element of $n$-body phase space. Since we are interested $t \bar{t}$ production and decay, with six particles in the final state, we use $d \Phi_{6}$.

If we measured all of the parton-level quantities, $y$, in our detector $W(x ; y)$ would simply be a $\delta$-function and we could directly evaluate $P_{t \bar{t}}\left(x ; m_{t}\right)$. However, this is not the case. In particular, we do not observe the neutrino or incoming partons at all, and we observe the outgoing partons only indirectly as jets. We choose not to consider the jet energies well measured, instead using explicit jet energy transfer functions as described in Chapter 6. But, a number of other constraints are available.

Starting from the 32-dimensional phase space containing the $t \bar{t}$ production and decay we can reduce:

- $[-8]$ : We know the masses of the two incoming and six outgoing partons

- [-4]: Conservation of four-momentum between initial and final states.

- $[-8]$ : We assume the jet angles are well measured.

- $[-3]$ : We assume that the charged lepton energy and angles are well measured.

- $[-4]$ : We assume that we can approximate the transverse momentum of the incoming $q$ and $\bar{q}$ zero.

This leaves five undetermined variables. We can think of, for example, the four parton energies and the $z$ component of the neutrino momentum. So, from Equation 7.2 we will have a five-dimensional integral remaining.

Returning to our equations, from [17] we have

$$
d \Phi_{6}=\delta^{4}\left(q_{1}+q_{2}-\sum_{i=1}^{6} p_{i}\right) \prod_{i=1}^{6} \frac{d^{3} p_{i}}{(2 \pi)^{3} 2 E_{i}}
$$


where $p_{i}$ are the four-momenta of the six outgoing particles with energy $E_{i}$. So, taking this along with Equations 7.1 and 7.2, and incorporating our constraints, we have

$$
\begin{aligned}
P_{t \bar{t}}\left(x ; m_{t}\right) & =\frac{1}{\sigma\left(m_{t}\right)} \int \frac{(2 \pi)^{4}|M|^{2} d q_{1} d q_{2} f\left(q_{1}\right) f\left(q_{2}\right)}{4 \sqrt{\left(q_{1} \cdot q_{2}\right)^{2}-m_{1}^{2} m_{2}^{2}}} \prod_{i=1}^{6} \frac{d^{3} p_{i}}{(2 \pi)^{3} 2 E_{i}} \\
& \times \delta^{4}\left(q_{1}+q_{2}-\sum_{i=1}^{6} p_{i}\right) \delta\left(p_{l}^{\text {meas }}-p_{l}\right) \prod_{j=1}^{N_{\text {jets }}=4} W\left(E_{j}^{j e t}, E_{j}^{\text {parton }}\right) \delta\left(\Omega_{j}^{\text {jet }}-\Omega_{j}^{\text {parton }}\right)
\end{aligned}
$$

where the charged lepton four-momentum and the jet angle measurements, $\Omega_{j}^{j e t}$, contribute $\delta$ functions. We use two of the constraints from energy conservation to eliminate the neutrino $p_{T}$ integrals and the other two to eliminate the integrals over $q_{1}$ and $q_{2}$. Further, we ignore the incoming quark masses so that $\sqrt{\left(q_{1} \cdot q_{2}\right)^{2}-m_{1}^{2} m_{2}^{2}}=2\left|q_{1}\right|\left|q_{2}\right|$. So, we have

$$
\begin{aligned}
P_{t \bar{t}}\left(x ; m_{t}\right) & =\frac{1}{\sigma\left(m_{t}\right)} \int \frac{(2 \pi)^{4}|M|^{2} f\left(q_{1}\right) f\left(q_{2}\right)}{(2 \pi)^{18} 2^{9}\left|q_{1}\right|\left|q_{2}\right|} \frac{1}{E_{l}} \frac{d p_{\nu}^{z}}{E_{\nu}} \\
& \times \prod_{i=1}^{N_{\text {jets }}=4} \frac{d^{3} p_{i}}{E_{i}} W\left(E_{i}^{\text {jet }}, E_{i}^{\text {parton }}\right) \delta\left(\Omega_{i}^{\text {jet }}-\Omega_{i}^{\text {parton }}\right) .
\end{aligned}
$$

We will discuss the integration, but first let us consider the matrix element itself. We base our matrix element on the expression from Mahlon and Parke[71]. Switching to notation where our process is $q \bar{q} \rightarrow t \bar{t} \rightarrow W^{+} b W^{-} \bar{b} \rightarrow \bar{e} \nu b d \bar{u} \bar{b}$, the form we use for the matrix element, averaged over the initial quark colors and spins and summed over the final colors and spins, is

$$
\sum|M|^{2}=\frac{g_{s}^{4}}{9} F \bar{F}\left(2-\beta^{2} s_{q t}^{2}\right)
$$

where $g_{s}$ is the strong coupling constant, $\beta$ is the velocity $(v / c)$ of the $t$ in the $q \bar{q}$ center of mass reference frame, and $s_{q t}$ is the sine of the angle between $q$ and $t$ in that same reference frame. Here we will make the approximation of neglecting the spin correlations, though Mahlon and Parke include them.

The factors $F$ and $\bar{F}$ come from the decay of the top quark $\left(t \rightarrow W^{+} b \rightarrow \bar{e} \nu b\right)$ and anti-top quark $\left(\bar{t} \rightarrow W^{-} \bar{b} \rightarrow d \bar{u} \bar{b}\right)$, respectively. Relative to Mahlon and Parke we restore the full top quark propagator, giving

$$
F=\frac{g_{w}^{4}}{4} \frac{\left(m_{t}^{2}-m_{\bar{e} \nu}^{2}\right)}{\left(m_{t}^{2}-M_{t}^{2}\right)^{2}+\left(M_{t} \Gamma_{t}\right)^{2}} \frac{m_{t}^{2}\left(1-\hat{c}_{\bar{e} b}^{2}\right)+m_{\bar{e} \nu}^{2}\left(1+\hat{c}_{\bar{e} b}\right)^{2}}{\left(m_{\bar{e} \nu}^{2}-M_{W}^{2}\right)^{2}+\left(M_{W} \Gamma_{W}\right)^{2}}
$$


where $g_{w}$ is the weak coupling constant, $m_{t}$ and $m_{w}$ represent kinematic mases, $M_{t}$ and $M_{W}$ represent pole masses, and $\hat{c}_{i j}$ is the cosine of the angle between particles $i$ and $j$ in the $W$ rest frame. The pole mass is the central value of the Breit-Wigner line shape expected for a given particle (approximately $80.4 \mathrm{GeV} / c^{2}$ for the $W$ ) while the kinematic mass is the mass of a particular $W$ boson, which varies form the pole mass according to the width of the Breit-Wigner (approximately $2 \mathrm{GeV} / c^{2}$ for the $W$ ). It is the kinematic mass that you would measure from one particular event where you knew all the parton four-momenta, but it is the pole mass that we are interested in determining from our ensemble of events.

For the hadronic side, knowing that we will soon sum the probability over all jetparton permutations, we perform a bit of symmetrization. The matrix element is nearly identical under exchange of the two quarks from the hadronic $W$ decay. We replace Mahlon and Parke's $m_{d \bar{u}}^{2}\left(1+\hat{c}_{d \bar{b}}\right)^{2}$ with $m_{d \bar{u}}^{2}\left(1+\hat{c}_{d \bar{b}}^{2}\right)$ and so implicitly sum over the jet-parton permutations that differ merely in the exchange of the two quarks from $W$ decay, since $\hat{c}_{d \bar{b}}=-\hat{c}_{\bar{u} \bar{b}}$. So, for the hadronic contribution we have

$$
\bar{F}=\frac{g_{w}^{4}}{4} \frac{\left(m_{t}^{2}-m_{d \bar{u}}^{2}\right)}{\left(m_{t}^{2}-M_{t}^{2}\right)^{2}+\left(M_{t} \Gamma_{t}\right)^{2}} \frac{m_{t}^{2}\left(1-\hat{c}_{d \bar{b}}^{2}\right)+m_{d \bar{u}}^{2}\left(1+\hat{c}_{d \bar{b}}^{2}\right)}{\left(m_{d \bar{u}}^{2}-M_{W}^{2}\right)^{2}+\left(M_{W} \Gamma_{W}\right)^{2}} .
$$

Now, we can see that the shape of the matrix element is driven by the top and $W$ Breit-Wigners. Since we intend to perform the integral with Monte Carlo methods, it makes sense to change to these variables where the integrand peaks sharply, specifically the top and $W$ invariant (pole) masses. But first we will change the jet integrals from Cartesian to spherical coordinates, $d^{3} p_{i} \rightarrow \rho_{i}^{2} d \rho_{i} d \Omega_{i}$, so that Equation 7.5 becomes

$$
P_{t \bar{t}}\left(x ; m_{t}\right)=\frac{1}{\sigma\left(m_{t}\right)} \int \frac{(2 \pi)^{4}|M|^{2} f\left(q_{1}\right) f\left(q_{2}\right)}{(2 \pi)^{18} 2^{9}\left|q_{1}\right|\left|q_{2}\right|} \frac{1}{E_{l}} \frac{d p_{\nu}^{z}}{E_{\nu}} \prod_{i=1}^{N_{\text {jets }}=4} \frac{\rho_{i}^{2} d \rho_{i}}{E_{i}} W\left(E_{i}^{\text {jet }}, E_{i}^{\text {parton }}\right)
$$

with our five integrals being over four jet energies $\left(\rho_{i}\right)$ and the neutrino $p_{z}$.

Next, we would like to change variables from $\left(\rho_{j_{1}}, p_{\nu}^{z}, \rho_{j_{2}}, \rho_{b_{h a d}}, \rho_{b_{l e p}}\right)$ to $\left(\rho_{j_{1}}, p_{\nu}^{z}, m_{W_{h a d}}, m_{t_{h a d}}^{2}, m_{t_{l e p}}^{2}\right)$ where $j_{1}$ and $j_{2}$ are the quarks from the hadronically decaying $W$. This will introduce a Jacobian term

$$
J=\left|\begin{array}{lll}
\frac{\delta m_{W_{\text {had }}}}{\delta \rho_{j_{2}}} & \frac{\delta m_{W_{\text {had }}}}{\delta \rho_{b_{\text {had }}}} & \frac{\delta m_{W_{\text {had }}}}{\delta \rho_{b_{l}}} \\
\frac{\delta m_{t_{\text {had }}}^{2}}{\delta \rho_{j_{2}}} & \frac{\delta m_{t_{\text {had }}}^{2}}{\delta \rho_{b_{h a d}}} & \frac{\delta m_{t_{\text {had }}}}{\delta \rho_{b_{l e p}}} \\
\frac{\delta m_{t_{l e p}}^{2}}{\delta \rho_{j_{2}}} & \frac{\delta m_{t_{l e p}}^{2}}{\delta \rho_{\rho_{\text {had }}}} & \frac{\delta m_{t_{l e p}}^{2}}{\delta \rho_{b_{l e p}}}
\end{array}\right| .
$$


Ignoring the light quark $\left(j_{2}\right)$ mass this becomes

$$
\begin{aligned}
J=\mid\left(\frac{E_{j_{1}}-\rho_{j_{1}} \cos \theta_{j_{1} j_{2}}}{m_{W}}\right) 2 & \left.\frac{E_{j_{2}} \rho_{b_{h a d}}}{E_{b_{h a d}}}+\frac{E_{j_{1}} \rho_{b_{h a d}}}{E_{b_{h a d}}}-\rho_{j_{1}} \cos \theta_{j_{1} b_{h a d}}-\rho_{j_{2}} \cos \theta_{j_{2} b_{h a d}}\right) \\
& \times 2\left(\frac{E_{l} \rho_{b_{l e p}}}{E_{b_{l e p}}}+\frac{E_{\nu} \rho_{b_{l e p}}}{E_{b_{l e p}}}-\rho_{\nu} \cos \theta_{\nu b_{l e p}}-\rho_{l} \cos \theta_{l b_{l e p}}\right) \mid
\end{aligned}
$$

and applying this change of variables to Equation 7.9 gives

$$
\begin{aligned}
P_{t \bar{t}}\left(x ; m_{t}\right) & =\frac{1}{\sigma\left(m_{t}\right)} \int \frac{|M|^{2} f\left(q_{1}\right) f\left(q_{2}\right)}{(2 \pi)^{14} 2^{9}\left|q_{1}\right|\left|q_{2}\right|} \frac{1}{E_{l}} \frac{d p_{\nu}^{z}}{E_{\nu}} \frac{1}{J} \\
& \times \prod_{i=1}^{N_{\text {jets }}=4} \frac{\rho_{i}^{2} d \rho_{j_{1}}}{E_{i}} W\left(E_{i}^{\text {jet }}, E_{i}^{\text {parton }}\right) d m_{W_{h a d}} d m_{t_{\text {had }}}^{2} d m_{t_{l e p}}^{2} .
\end{aligned}
$$

So, we still have five integrals, but with integration variables chosen to facilitate rapid integration with Monte Carlo methods.

Next, to simplify the Monte Carlo integration even further, we employ a narrow width approximation to the two $m_{t}^{2}$ integrals and Breit-Wigners (see, e.g., Ellis, Stirling, and Weber [72])

$$
\frac{1}{\left(m_{t}^{2}-M_{t}^{2}\right)^{2}+M_{t}^{2} \Gamma_{t}^{2}} \approx \frac{\pi}{M_{t} \Gamma_{t}} \delta\left(m_{t}^{2}-M_{t}^{2}\right) .
$$

Under this approximation, Equations 7.7 and 7.8 become

$$
F=\frac{g_{w}^{4}}{4} \frac{\pi\left(M_{t}^{2}-m_{\bar{e} \nu}^{2}\right)}{M_{t} \Gamma_{t}} \frac{M_{t}^{2}\left(1-\hat{c}_{\bar{e} b}^{2}\right)+m_{\bar{e} \nu}^{2}\left(1+\hat{c}_{\bar{e} b}\right)^{2}}{\left(m_{\bar{e} \nu}^{2}-M_{W}^{2}\right)^{2}+\left(M_{W} \Gamma_{W}\right)^{2}}
$$

and

$$
\bar{F}=\frac{g_{w}^{4}}{4} \frac{\pi\left(M_{t}^{2}-m_{d \bar{u}}^{2}\right)}{M_{t} \Gamma_{t}} \frac{M_{t}^{2}\left(1-\hat{c}_{d \bar{b}}^{2}\right)+m_{d \bar{u}}^{2}\left(1+\hat{c}_{d \bar{b}}^{2}\right)}{\left(m_{d \bar{u}}^{2}-M_{W}^{2}\right)^{2}+\left(M_{W} \Gamma_{W}\right)^{2}} .
$$

Incidentally, we also take into account the $m_{t}$-dependence of $\Gamma_{t}$, following in the style of Canelli [70]. Applying a narrow width approximation to the $W$ Breit-Wigner in the expression for top decay, we have

$$
\Gamma_{t}=\frac{g_{w}^{4} M_{t}^{3} \bar{\pi}}{2^{7}(2 \pi)^{3} 3} \frac{1-3\left(\frac{M_{W}}{M_{t}}\right)^{4}+2\left(\frac{M_{W}}{M_{t}}\right)^{6}}{M_{W} \Gamma_{W}}
$$

with

$$
\bar{\pi}=\arctan \left[\frac{\left(M_{t}-M_{b}\right)^{2}-M_{W}^{2}}{M_{W} \Gamma_{W}}\right]-\arctan \left[\frac{-M_{W}^{2}}{M_{W} \Gamma_{W}}\right] .
$$

As we have mentioned, since in general we cannot attach unique parton assignments to the jets we must consider all jet-parton permutations. Since we are considering 
four-jet events there are, in general, 24 permutations. In symmetrizing $F$ we have reduced this number to 12 . Owing to the roots of this analysis as a pre-tag measurement we sum over all twelve of these combinations. In a future version of this analysis we plan to also take advantage of the $b$ tagging information to reduce the number of combinations considered to six for an event with one $b$ tag and just two for an event with two $b$ tags. As well as the jet-parton permutations, we must also sum over the multiple possible solutions to the kinematic equations. As we will discuss below, there we consider up to four kinematic solutions at each point in the phase space of the integral. So, the final form of our probability, becomes

$$
\begin{aligned}
P_{t \bar{t}}\left(x ; m_{t}\right)=\frac{1}{\sigma\left(m_{t}\right)} \int d p_{\nu}^{z} d \rho_{j_{1}} d m_{W_{\text {had }}} & \\
& \times \sum_{\text {comb,soln's }} \frac{|M|^{2} f\left(q_{1}\right) f\left(q_{2}\right)}{(2 \pi)^{14} 2^{9}\left|q_{1}\right|\left|q_{2}\right|} \frac{1}{E_{l}} \frac{1}{E_{\nu}} \frac{1}{J} \prod_{i=1}^{N_{j e t s}=4} \frac{\rho_{i}^{2}}{E_{i}} W\left(E_{i}^{\text {jet }}, E_{i}^{\text {parton }}\right)
\end{aligned}
$$

For the $m_{t}$-dependent shape of $\sigma\left(m_{t}\right)$ we use a parameterized curve fit to cross section values from leading-order PYTHIA $t \bar{t}$ Monte Carlo, generated with no radiation or hadronization. This calculation mimics what we would expect from the analytically expressed leading-order differential cross section in $P_{t} \bar{t}$. Since it can be difficult to keep track of all of the factors of $2, \pi$, etc. in $P_{t \bar{t}}$ we have performed a 15-D Monte Carlo integral of $P_{t \bar{t}}$ over the entire phase space of measured variables. The result of this calculation could be used as a normalization for $P_{t \bar{t}}$ and then be used with a correctly normalized $P_{b a c k}$ in our overall likelihood (Equation 5.8). However, as we will discuss in Chapter 8, we handle the normalization of our overall likelihood empirically. So, it is not important that $P_{t \bar{t}}$ itself have a physically meaningful overall normalization. A missing mass-dependence in the normalization would be significant, but this is taken care of the parameterized curve from PYTHIA. So, the $P_{t \bar{t}}$ we use in practice has an arbitrary (but $m_{t}$-independent) multiplicative factor included, and thus the absolute scale has no immediate physical interpretation.

So, in Equation 7.18 we are left with three integrals to perform, and the variables are the neutrino $p_{z}$, the energy of one quark from $W$ decay, and the kinematic mass of the hadronically decaying $W$. We perform the three-dimensional integration using VEGAS [73], a standard adaptive-grid Monte Carlo integration code. The particular implementation we use is that of the GNU Scientific Library [74]. We evaluate the integral for specific values of $m_{t}$, in $1 \mathrm{GeV} / c^{2}$ steps, from $130-230 \mathrm{GeV} / c^{2}$. At each point in the integral we know the 
values of $m_{t}, m_{W_{h a d}}, p_{\nu}^{z}$, and $\rho_{j_{1}}\left(\right.$ i.e. $\left.\left|\vec{p}_{j_{1}}\right|\right)$, as well as the angles of the charged lepton and all jets. From $m_{W_{h a d}}$ and $\rho_{j_{1}}$ it is easy to solve for $\rho_{j_{2}}$. Then, with $m_{t}$ it is easy to solve for $\rho_{b_{\text {had }}}$. On the leptonic side things are a little more complex. To facilitate a closed-form algebraic solution we make the approximation, as part of the kinematic solutions only, that the mass of the leptonic $b$ quark is zero. Then, there are up to four algebraic solutions for $\rho_{b_{l e p}}$, and the rest of the event kinematics follow easily. With the full event kinematics in hand, at each point in the phase space of the integral we simply evaluate the transfer functions, the matrix element, and the rest of $P_{t \bar{t}}$.

\subsection{Exploring the Signal Probability}

The results of the $P_{t \bar{t}}$ calculation for twenty signal events $\left(m_{t}=178 \mathrm{GeV} / c^{2}\right.$ from fully simulated HERWIG $t \bar{t}$ events) can be found in Figure 7.1. These curves are actually not quite $P_{t \bar{t}}$, as the $\sigma\left(m_{t}\right)$ normalization has not yet been applied. And, as we have discussed, the overall vertical scale is arbitrary. Since we will multiply these curves to form a joint likelihood curve, multiplying an individual curve by a constant will have no effect on the measured mass. What affects how much a particular curve contributes to the likelihood is how sharply peaked the curve is, and, of course, where it peaks. For a closer look, two of the events are shown separately in Figure 7.2.

One simple test of the convergence of the integration procedure is to examine the smoothness of the probability for a given event. The true probability is expected to be a smooth function of $m_{t}$; since each point is integrated independently, the variation from a smooth function indicates the level of convergence of the integration.

We can take these twenty events and construct a pseudo-experiment from which to extract a best-fit $m_{t}$. Using a signal-only likelihood (Equation 5.8 with $c_{1}$ set to 1.0) we construct a $-\ln L$ curve as can be seen in Figure 7.3. We fit this curve to a parabola, fitting a range $10 \mathrm{GeV} / c^{2}$ to either side of the maximum likelihood mass. The best-fit $m_{t}$ is given by the minimum of the parabola, and the naively estimated error is given by the distance

to the mass which gives $\Delta \ln L=\frac{1}{2}$. As we will discuss shortly, to accurately estimate the error we will need to inflate this naive error from $\Delta \ln L=\frac{1}{2}$. For this particular set of 20 events we measure $m_{t}=175.1 \mathrm{GeV} / c^{2}$ and the naively estimated error is $3.4 \mathrm{GeV} / c^{2}$. 

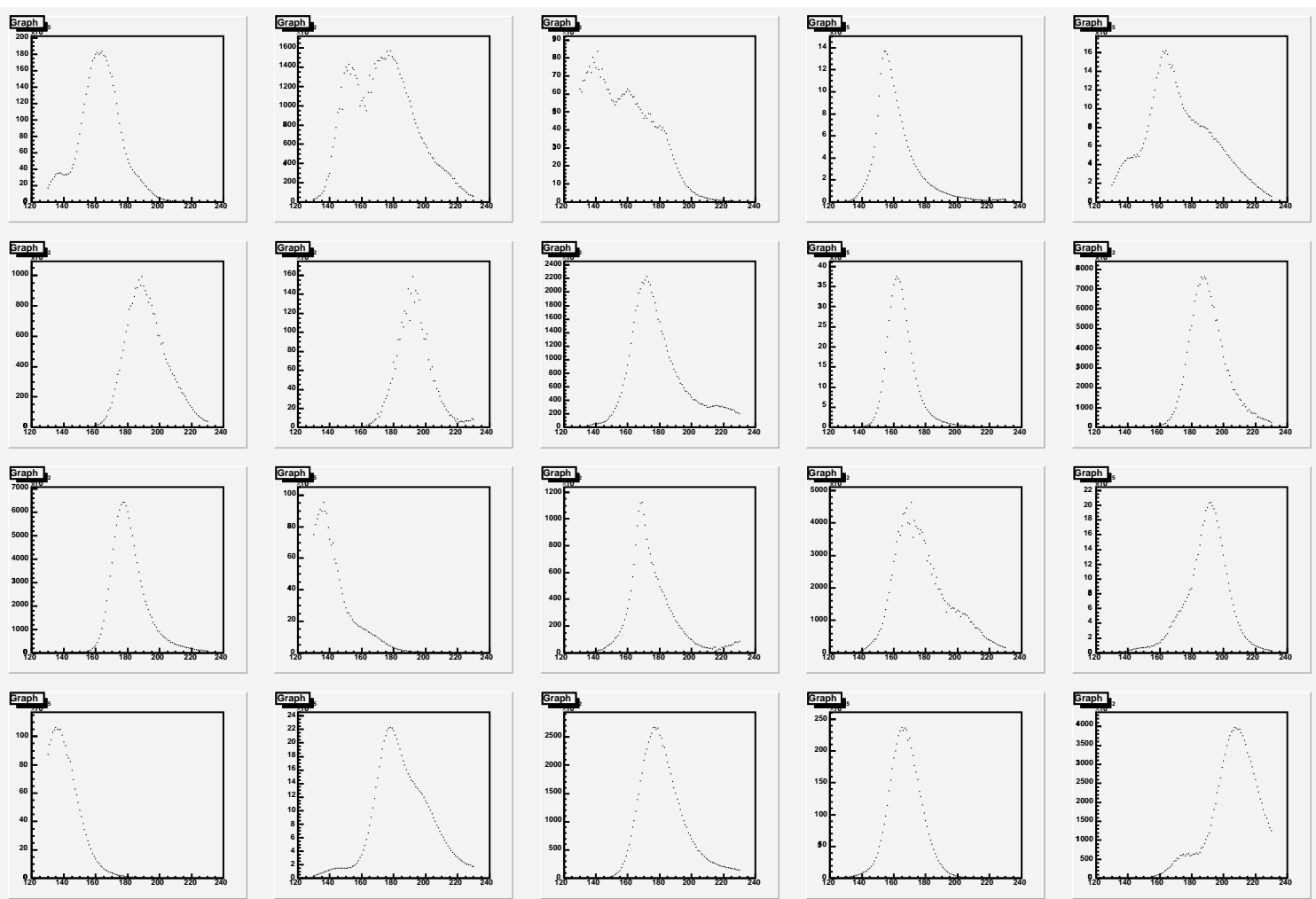

Figure 7.1: Unnormalized signal probability for each of twenty fully simulated HERWIG $(t \bar{t}$ events, $m_{t}=178 \mathrm{GeV} / c^{2}$. The $y$ axis is proportional, apart from the missing $\sigma\left(m_{t}\right)$ factor, to $P_{t \bar{t}}$. The $x$ axis is $m_{t}$ from 130 to $230 \mathrm{GeV} / c^{2}$.
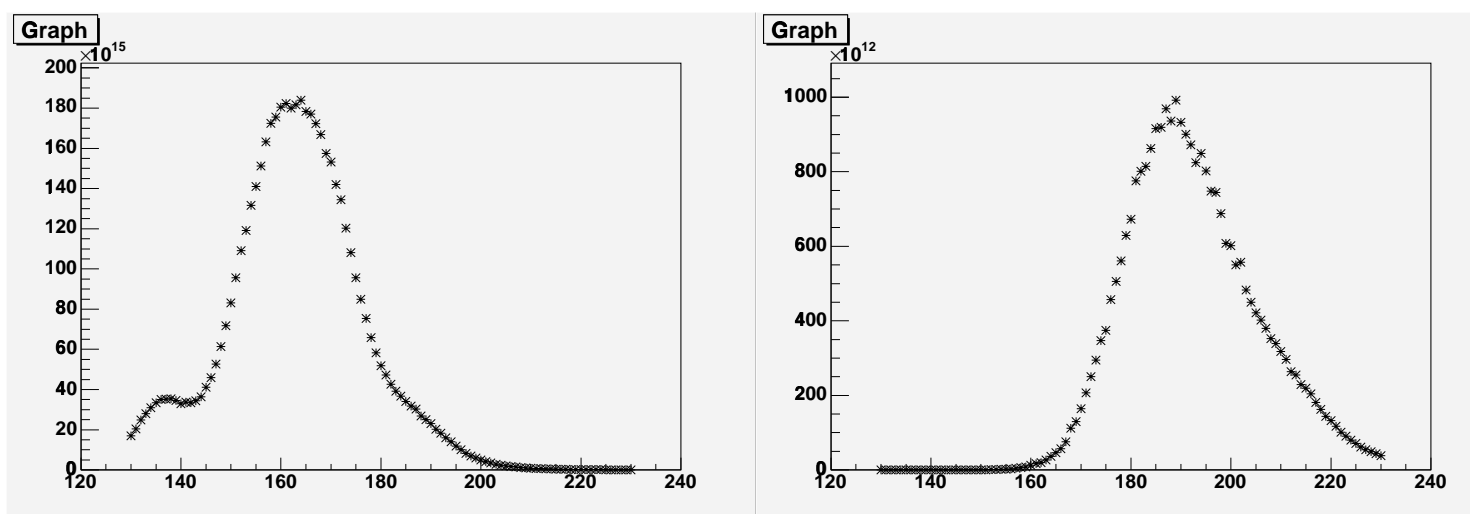

Figure 7.2: Closer view of two of the events from Figure 7.1. The $y$ axis is proportional, apart from the missing $\sigma\left(m_{t}\right)$ factor, to $P_{t \bar{t}}$. 

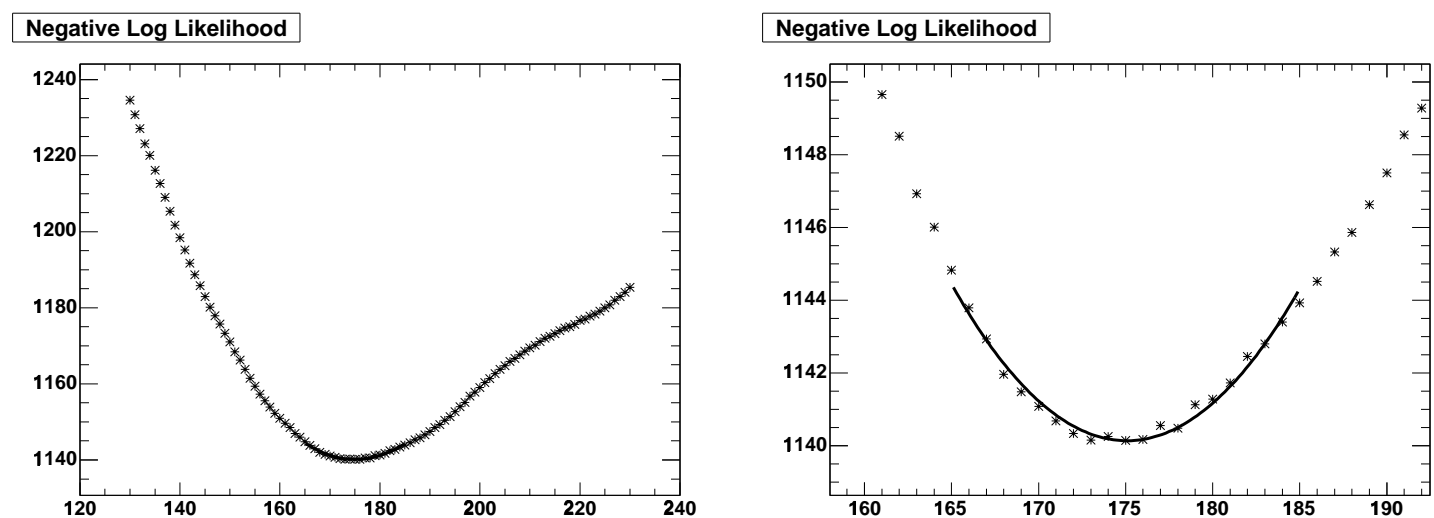

Figure 7.3: Negative log likelihood curve for the twenty events in Figure 7.1. The normalization is arbitrary, but all mass-dependent corrections have been applied. The $x$ axis is $m_{t}$ from 130 to $230 \mathrm{GeV} / c^{2}$. The mean is $175.1 \mathrm{GeV} / c^{2}$ and the naively estimated error is $3.4 \mathrm{GeV} / c^{2}$, both extracted from the fitted parabola. The right-hand plot is simply a zoomed-in version of the left-hand.

\subsection{Additional Event Selection}

A small fraction of events behave badly in our $P_{t \bar{t}}$ calculation, leading us to reject them with an analysis-specific event selection requirement. There are two related pathologies.

First, for a small fraction of events $\left(0.5 \%\right.$ for $178 \mathrm{GeV} / c^{2} t \bar{t}$ evevnts, $0.8 \%$ for background samples) our $P_{t \bar{t}}$ code simply fails to converge for one or more of the pole masses (130-230 GeV/ $\left./ c^{2}\right)$ considered. This can happen because the integration is converging too slowly to reasonably finish the calculation, because the value of the integral toward which the code is converging is too small to handle, or because of a failure to find kinematic solutions. These events need to be vetoed in order to avoid discontinuities in our joint likelihood distribution.

Second, some events produce pathological $P_{t \bar{t}}$ shapes for related reasons. The code does not fail to converge, but produces extremely low values for part of the $m_{t}$ space considered. This leads to events with extreme $P_{t \bar{t}}$ shapes which, when multiplied with other probability curves in a signal-only likelihood (a likelihood with only $P_{t \bar{t}}$ information), pathologically distort the results.

For example, consider the $P_{t \bar{t}}$ distribution, shown in Figure 7.4, of a single, pathological, fully simulated Monte Carlo $t \bar{t}$ event. This event does not have a $b$ tag, so would 


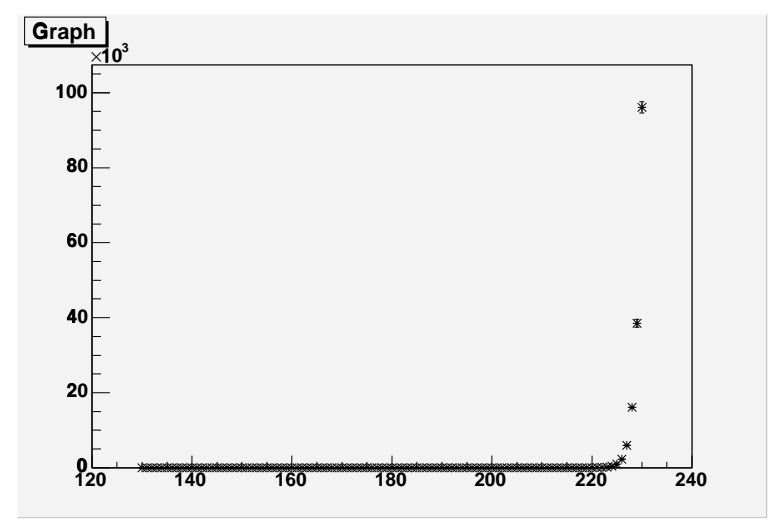

Figure 7.4: Pathological $t \bar{t}$ event which leads to a significant distortion of pseudoexperiments. The $y$ axis is proportional, apart from the missing $\sigma\left(m_{t}\right)$ factor, to $P_{t \bar{t}}$. The $x$ axis is $m_{t}$ from 130 to $230 \mathrm{GeV} / c^{2}$. The probability, for any $m_{t}$ is much lower than a typical event, but is extremely "shapely", varying across 100 orders of magnitude.

not form part of the dataset for this analysis. But, it is an interesting case study. For this event, our code never actually returns $P_{t \bar{t}}=0$, but the results range from $1 \times 10^{-96}$ at $130 \mathrm{GeV} / c^{2}$ to $1 \times 10^{-4}$ at $230 \mathrm{GeV} / c^{2}$. For typical events, peak $P_{t \bar{t}}$ values are in the range $1 \times 10^{-10}-1 \times 10^{-17}$. We construct pseudo-experiments ${ }^{1}$ by randomly selecting ensembles of 78 events from a large sample of simulated $t \bar{t}$ events, while allowing an event to participate in more than one ensemble (allowing resampling). The mean $m_{t}$ values from ensembles of such pseudo-experiments drawn from samples with and without this single pathological event are shown in Figure 7.5. Every pseudo-experiment that contains this event is biased toward a high $m_{t}$, forming a secondary peak around $195 \mathrm{GeV} / c^{2}$.

The effect of events like this would be ameliorated in the full signal + background likelihood, because adding even a modest background probability (e.g. $1 \times 10^{-1}$ ) would greatly reduce the "shapeliness" of the event, which currently varies across 100 orders of magnitude. But, it seems desirable to have $P_{t \bar{t}}$ results that can be trusted independently of $P_{b a c k}$. And, it would be a shame to have an event like that of Figure 7.4 pass our event selection and, for example, be included in our $m_{t}$ measurement in data. So, we established an additional event selection cut to avoid such events.

Finally, the effect of such events in the background samples seems even more pronounced. Events such as that of Figure 7.4 are more frequent than in $t \bar{t}$ events. This seems reasonable since $P_{t \bar{t}}$ is not intended to model background events. We chose an event

\footnotetext{
${ }^{1}$ We discuss our pseudo-experiment methodology in detail in Section 7.4.
} 

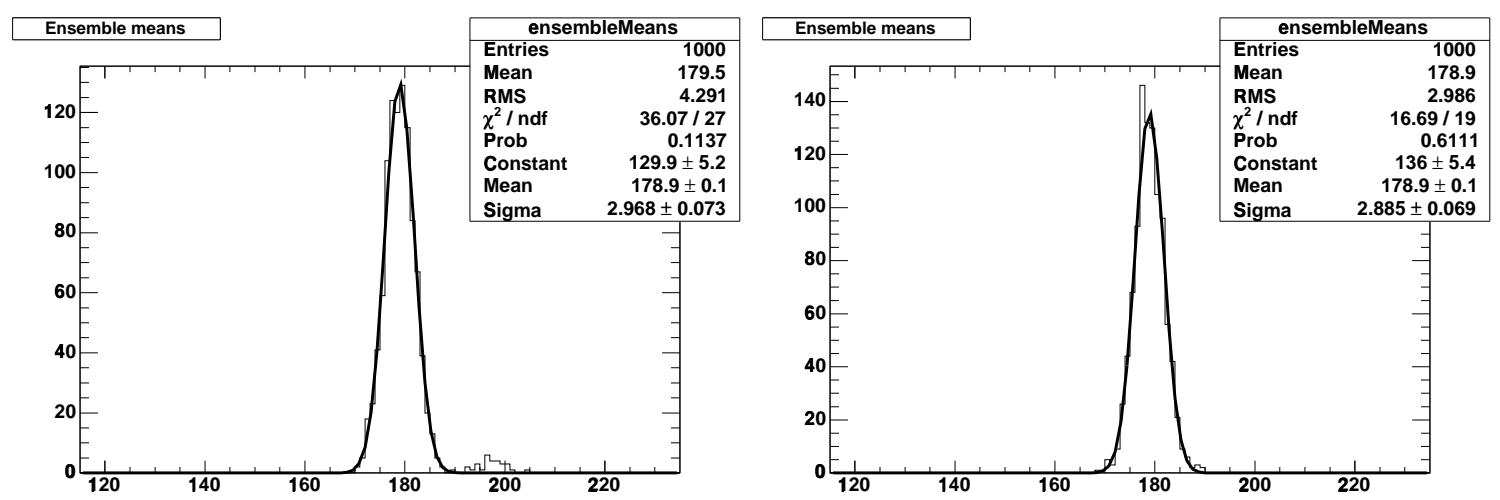

Figure 7.5: Means of 78-event resampled pseudo-experiments drawn from a sample of simulated $t \bar{t}$ events with (left) and without (right) the pathological event of Figure 7.4. Every pseudo-experiment that includes this event is biased high, and included in the second peak around $195 \mathrm{GeV} / c^{2}$. The $x$ axis is $m_{t}$ from 130 to $230 \mathrm{GeV} / c^{2}$.

selection cut designed to directly address the observed pathology. We remove events where the minimum $P_{t \bar{t}}$ (in the $130-230 \mathrm{GeV} / c^{2}$ region scanned) is less than $1 \times 10^{-1}$, in the arbitrary (but, of course, fixed) overall normalization that we have adopted. This is designed to eliminate anomalously shapely events caused by very low signal probabilities. It is effectively equivalent to raising the threshold, internal to our $P_{t \bar{t}}$ code, below which our code gives up on calculating the integral. So, we are effectively moving these pathologically shapely events from the second category of failures to the first, those which are automatically rejected by our $P_{t \bar{t}}$ code.

We tune the value of this $P_{t \bar{t}}$ cut by considering two distributions. The first is the mean $m_{t}$ value of pseudo-experiments, which we have already seen. The second is the distribution of most probable $m_{t}$ values for each event. This is effectively a onedimensional mass template, and indicates the shape of the $m_{t}$ distribution if all events had unit weight (i.e. if the shape of the $P_{t \bar{t}}$ curve is ignored). We chose a cut that removed the pathological events (as indicated by the first distribution) while minimally biasing the second distribution, and while also attempting to minimize the number of $t \bar{t}$ events removed. The effect of this cut on simulated $178 \mathrm{GeV} / c^{2} t \bar{t}$ events on background samples can be seen in Table 7.1.

The overall acceptance for these two cuts $\left(P_{t \bar{t}}=0\right.$ removal and the $1 \times 10^{-1} P_{t \bar{t}}$ cut $)$ can be seen in Figure 5.2. The number of events removed for large $m_{t}$ is significant, so if we expected $m_{t}$ to be significantly above $178 \mathrm{GeV} / c^{2}$ we would re-consider the cut value. But, 


\begin{tabular}{|c|c|c|c|c|c|c|c|}
\hline Sample & $\begin{array}{c}\text { Events } \\
\text { before } \\
P_{t \bar{t}} \\
\text { cuts }\end{array}$ & $\begin{array}{c}\text { Events } \\
\text { after } \\
0 \text { cut }\end{array}$ & $\begin{array}{c}\text { Events } \\
\text { after } \\
1 \times 10^{-1} \\
\text { cut }\end{array}$ & $\begin{array}{c}\text { max L } \\
\text { mean } \\
\text { pre-cut } \\
\left(\mathrm{GeV} / c^{2}\right)\end{array}$ & $\begin{array}{c}\text { max L } \\
\text { mean } \\
\text { post-cut } \\
\left(\mathrm{GeV} / c^{2}\right)\end{array}$ & $\begin{array}{c}\text { mean } \\
\text { from PE } \\
\text { pre-cut } \\
\left(\mathrm{GeV} / c^{2}\right)\end{array}$ & $\begin{array}{c}\text { mean } \\
\text { from PE } \\
\text { post-cut } \\
\left(\mathrm{GeV} / c^{2}\right)\end{array}$ \\
\hline$t \bar{t}$ & 4347 & 4326 & 4239 & 174.3 & 173.5 & 179.5 & 177.6 \\
\hline$W(\mu)+4 p$ & 1697 & 1679 & 1590 & 166.9 & 163.8 & 180.5 & 164.4 \\
\hline$W(e)+4 p$ & 1550 & 1541 & 1481 & 166.0 & 163.7 & 174.0 & 164.1 \\
\hline$W(e) b \bar{b}$ & 422 & 417 & 393 & 169.1 & 166.0 & 180.7 & 166.1 \\
\hline$W(\mu) b \bar{b}$ & 535 & 530 & 501 & 167.2 & 164.3 & 177.6 & 167.6 \\
\hline$W(e) c \bar{c}$ & 150 & 149 & 142 & 167.1 & 164.6 & 176.7 & 165.3 \\
\hline$W(\mu) c \bar{c}$ & 160 & 158 & 149 & 166.0 & 163.4 & 175.4 & 163.1 \\
\hline$W c$ & 256 & 254 & 247 & 163.8 & 162.5 & 167.7 & 159.3 \\
\hline Single $t(\mathrm{~s})$ & 408 & 405 & 393 & 171.3 & 169.7 & 174.8 & 170.0 \\
\hline Single $t(\mathrm{t})$ & 282 & 281 & 277 & 170.0 & 169.3 & 173.1 & 171.7 \\
\hline$Z Z$ & 13 & 13 & 12 & 173.5 & 168.8 & 185.6 & 169.1 \\
\hline$W W$ & 28 & 28 & 28 & 153.7 & 153.7 & 152.3 & 152.3 \\
\hline$W Z$ & 48 & 47 & 45 & 161.5 & 158.5 & 164.7 & 159.6 \\
\hline $\mathrm{QCD}$ & 43 & 43 & 43 & 171.1 & 171.1 & 169.0 & 169.0 \\
\hline $\mathrm{QCD}$ & 33 & 33 & 30 & 172.3 & 166.9 & 197.2 & 173.8 \\
\hline
\end{tabular}

Table 7.1: Fully simulated $178 \mathrm{GeV} / c^{2} t \bar{t}$ and background samples before and after cuts related to $P_{t \bar{t}}$. The " 0 cut" is the removal of events with one or more $m_{t}$ points of $P_{t \bar{t}}=0$. All of the mass values listed, the four rightmost columns, include this cut. The " $1 \times 10^{-1}$ cut" is the cut to remove pathological events that distort pseudo-experiments (PE's). The mean of the distribution of maximum likelihood masses gives an indication of the preferred mass if all events are given unit weight. The mean of the mass from pseudo-experiments (here they are 36.68 event pseudo-experiments) is the result from the signal-only likelihood. For each row pseudo-experiments were constructed using events from that sample only. 
at $178 \mathrm{GeV} / c^{2}$ the number of events removed is modest (for $t \bar{t} 0.5 \%$ due to zeroes and $2.0 \%$ due to the $1 \times 10^{-1}$ cut; for background $0.8 \%$ due to zeroes and $5.2 \%$ due to the $1 \times 10^{-1}$ cut). And, the scale and $m_{t}$ dependence of this additional acceptance (Figure 5.2) is modest compared to the acceptance of the usual event selection (Figure 5.1). The acceptance of this cut, including $m_{t}$ dependence, is included along with all of the traditional cuts in the overall acceptance used in our likelihood (Equation 5.8).

\subsection{Pseudo-Experiment Methodology}

Even with the integration significantly simplified, computing the signal probability is computationally intensive. Computing $P_{t \bar{t}}$ for one event at $100 m_{t}$ points takes on order ten minutes on a reasonably modern personal computer. So, the the number of fully simulated Monte Carlo events that we can process with the $P_{t \bar{t}}$ code is not unlimited. Thus, for tests of our methods we sometimes employ statistical resampling. When estimating the value of quantities such as the mean $m_{t}$ result from an ensemble of pseudo-experiments we do not expect resampling to improve the uncertainty of our estimate. But, when estimating quantities such as the width of pull distributions we do expect resampling to improve our uncertainty. It has been suggested that resampling can improve the uncertainty of such quantities when using as many as $N_{\text {exclusive }}^{2}$ pseudo-experiments, where $N_{\text {exclusive }}$ is the number of pseudo-experiments available without resampling. To be conservative, we assume that we can benefit from no more than $\frac{1}{3}$ this number of pseudo-experiments.

In general, we extract most of our test results from heavily resampled pseudoexperiments. For quantities such as the mean $m_{t}$ we take the central value from a Gaussian fit of the resampled pseudo-experiments. Since resampling should not decrease the uncertainty on the mean $m_{t}$ value of the ensemble of pseudo-experiments we may not rely simply on the error on the fitted mean $m_{t}$. It will be artificially small. Instead, we estimate the uncertainty as $\frac{\sigma}{\sqrt{N_{\text {exclusive }}}}$ where $\sigma$ is the width of the $m_{t}$ distribution, taken from the Gaussian fit. This is the uncertainty we would expect based upon the number of possible exclusive pseudo-experiments. For quantities such as the width of pull distributions we also take the central value from a Gaussian fit of the resampled pseudo-experiments. We estimate the uncertainty as $\frac{\sigma}{\sqrt{\frac{1}{3} N_{\text {exclusive }}^{2}}}$. As we will discuss further below, this uncertainty seems to be underestimated, at least in some cases. But, the uncertainty on the mean seems robust. It seems worth noting that the error on a quantity improved by resampling in no way enters 
our calculation of the top mass, or the uncertainty on the top mass. The error on such quantities is only helpful for diagnostic purposes.

We form our pseudo-experiments using events from $t \bar{t}$ samples and from one or more backgrounds. The number of events chosen from each sample is Poisson-fluctuated independently about its mean. We will consider pseudo-experiments with two different comparisons in mind. For comparison with other analyses, we construct pseudo-experiments assuming a $6.1 \mathrm{pb} t \bar{t}$ cross section. With our acceptance and integrated luminosity this is equivalent to 35.94 events, as discussed in Table 4.3. So, these $6.1 \mathrm{pb}$ pseudo-experiments contain 35.94 Poisson-mean $t \bar{t}$ events. But, we have 63 events in our actual dataset. So, for comparison to data we use pseudo-experiments with 54.33 Poisson-mean $t \bar{t}$ events. When we add background events to our pseudo-experiments they are added according to Table 4.3.

\subsection{Tests of Signal-Only Likelihood with $t \bar{t}$ Pseudo- Experiments}

Now, with our tools and methodology in place we can look at results of a signalonly likelihood (using $P_{t \bar{t}}$ but not $P_{b a c k}$ ) for $t \bar{t}$ events. In this section all pseudo-experiments are constructed with the assumption of a $6.1 \mathrm{pb} t \bar{t}$ cross section, and with no background events included.

First, we consider pseudo-experiments drawn from a sample of fully simulated $t \bar{t}$ events with $m_{t}=178 \mathrm{GeV} / c^{2}$. For each pseudo-experiment we fit a parabola to the $-\ln L$ curve and extract a mean $m_{t}$ with a naively estimated error from $\Delta \ln L=\frac{1}{2}$. Distributions of the measured $m_{t}$ and the naively estimated error from such an ensemble of pseudoexperiments can be found in Figure 7.6.

The pulls (where we define pull as $\frac{m_{t}^{f i t}-m_{t}^{\text {true }}}{\text { estimatederror }}$ ) can be found in Figure 7.7. If $m_{t}$ is correctly estimated we expect the mean of the pull distribution to be zero. If the error on $m_{t}$ is correctly estimated we expect the width of the pull distribution to be unity. It is obvious that the width of the pull distribution is significantly greater than one, which is consistent with the width of the distribution of fitted mean $m_{t}$ 's being larger than the mean of the distribution of naively estimated errors. That is, the naively estimated error

from $\Delta \ln L=\frac{1}{2}$ underestimates the actual error on $m_{t}$. This is because a number of the assumptions we make in formulating our likelihood are broken in these fully simulated 

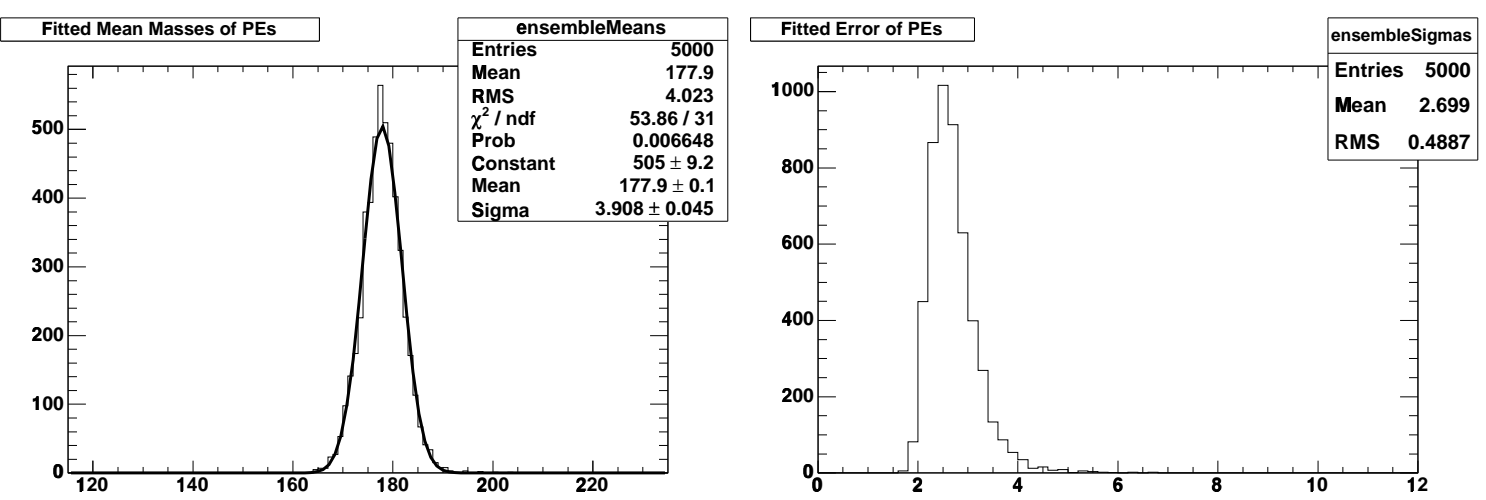

Figure 7.6: Means (left) and estimated, uncorrected errors $\left(\Delta \ln L=\frac{1}{2}\right)$ (right) from 35.94 event resampled pseudo-experiments drawn from $178 \mathrm{GeV} / c^{2} t \bar{t}$ events and analyzed with the signal-only likelihood. The $x$ axis is $m_{t}$ in $\mathrm{GeV} / c^{2}$.

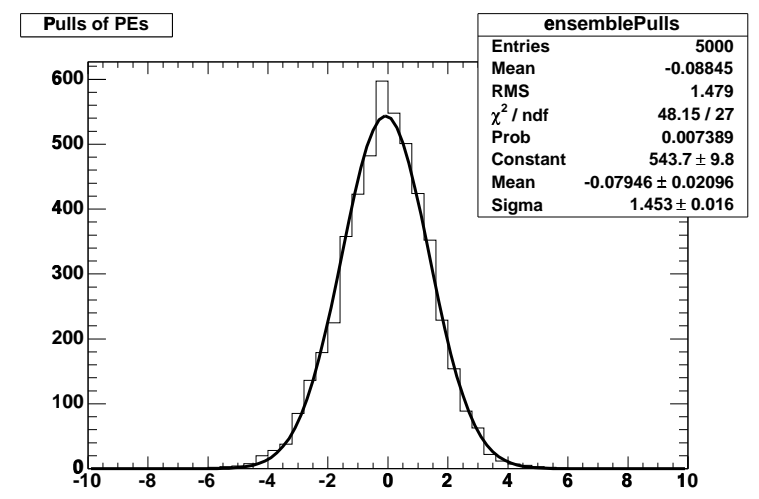

Figure 7.7: Pulls from 35.94 event resampled pseudo-experiments drawn from $178 \mathrm{GeV} / c^{2}$ $t \bar{t}$ events and analyzed with the signal-only likelihood. The pull of a pseudo-experiment is defined as $\frac{m_{t}^{f i t}-m_{t}^{\text {true }}}{\text { estimatederror }}$.

events. We will discuss this phenomenon in detail in Section 12.1.

Next, we construct similar ensembles of pseudo-experiments from a wide variety of top mass samples. We plot the measured $m_{t}$ values from these ensembles in Figure 7.8, and the width of the pull distributions in Figure 7.9. The plot on the left of Figure 7.8 is often referred to as a linearity plot since an unbiased measurement of the top mass would have a slope of unity. Figure 7.8 is not consistent with having a slope of unity, but it is not too far off. This indicates that our signal-only likelihood can measure $m_{t}$ over a wide range of masses, but that at low masses a correction as large as $1.5 \mathrm{GeV} / c^{2}$ would be necessary. It is clear that the errors on the pull widths in Figure 7.9 are underestimated, as the $\chi^{2}$ per 

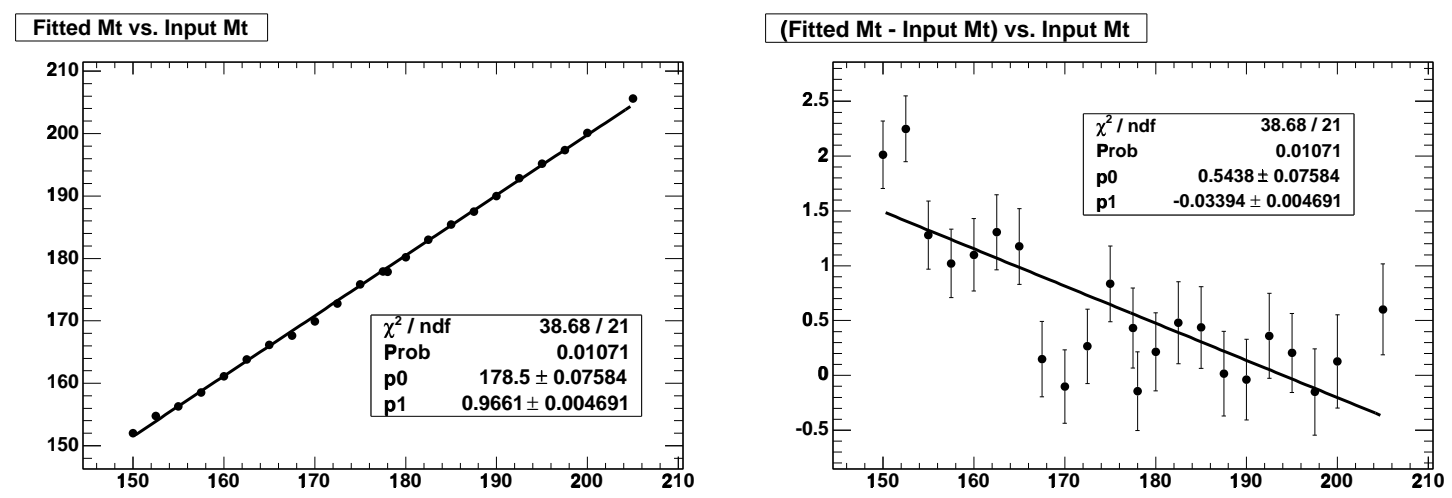

Figure 7.8: Fully simulated $t \bar{t}$ events analyzed with signal-only likelihood, 35.94 event pseudo-experiments. On the left is the usual linearity test, fitted $m_{t}$ vs. input $m_{t}$. On the right is the same information plotted as (fitted $m_{t}$ - input $m_{t}$ ) vs. input $m_{t}$. Both are fit to $\mathrm{p} 0+\mathrm{p} 1 *$ (input $m_{t}-178 \mathrm{GeV} / c^{2}$ ). All axes are in $\mathrm{GeV} / c^{2}$.

degree of freedom is unacceptable. But they are consistent with being flat as a function of $m_{t}$, having a fitted mean of 1.46 . So, if we wanted to make a reliable pseudo-experiment by pseudo-experiment estimate of the uncertainty of the fitted $m_{t}$ we would inflate the naively estimated error by $46 \%$.

For comparison with other cases, we note that the mean expected error at 178 $\mathrm{GeV} / c^{2}$, taken from the fitted with of the $m_{t}$ distribution in Figure 7.6 is $3.91 \mathrm{GeV} / c^{2}$. So, our expected error with the number of $t \bar{t}$ events we expect from a $6.1 \mathrm{pb} t \bar{t}$ cross section is $3.91 \mathrm{GeV} / c^{2}$. Here our pseudo-experiments and are likelihood each consider only $t \bar{t}$.

\subsection{Tests of Signal-Only Likelihood with Signal + Background Pseudo-Experiments}

Next, we prepare pseudo-experiments using the full set of expected background samples. For the number of signal events we again use the $6.1 \mathrm{pb}$ assumption, and so have 35.94 mean signal events per pseudo-experiment. The mean number of background events, for each background, is as in Table 4.3. Since we are introducing background events without modelling them (i.e. without including a background term in the likelihood) we expect that our $m_{t}$ measurement may be biased and that the width of our pull distributions may be inflated. We plot the measured $m_{t}$ values from these ensembles in Figure 7.10, and the width of pull distributions in 7.11 . 


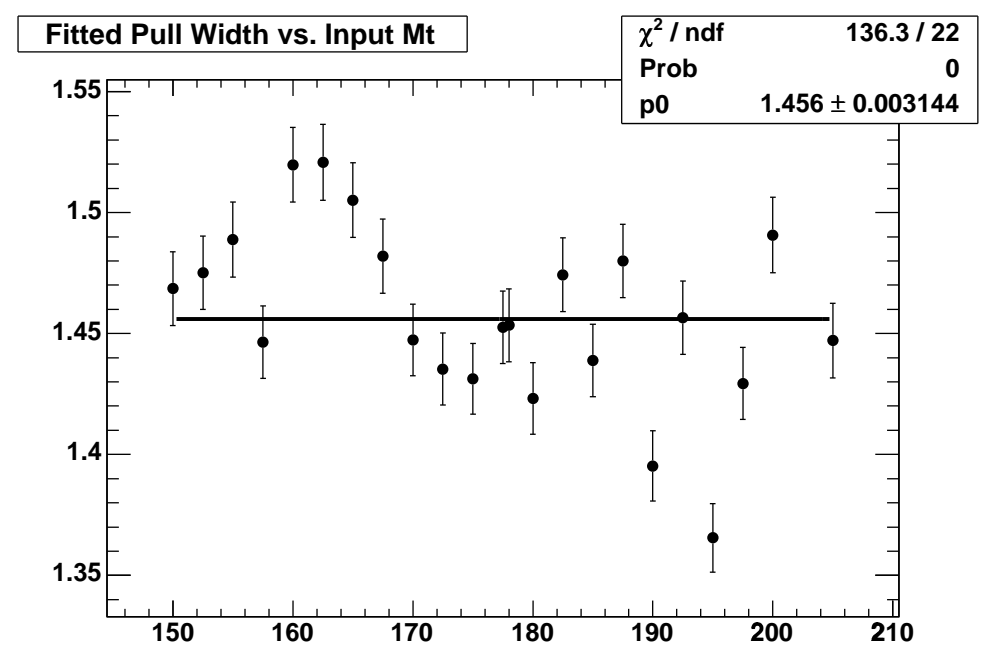

Figure 7.9: Fitted widths of pull distributions plotted vs input $m_{t}$. Fully simulated $t \bar{t}$ events analyzed with signal-only likelihood, 35.94 event pseudo-experiments.
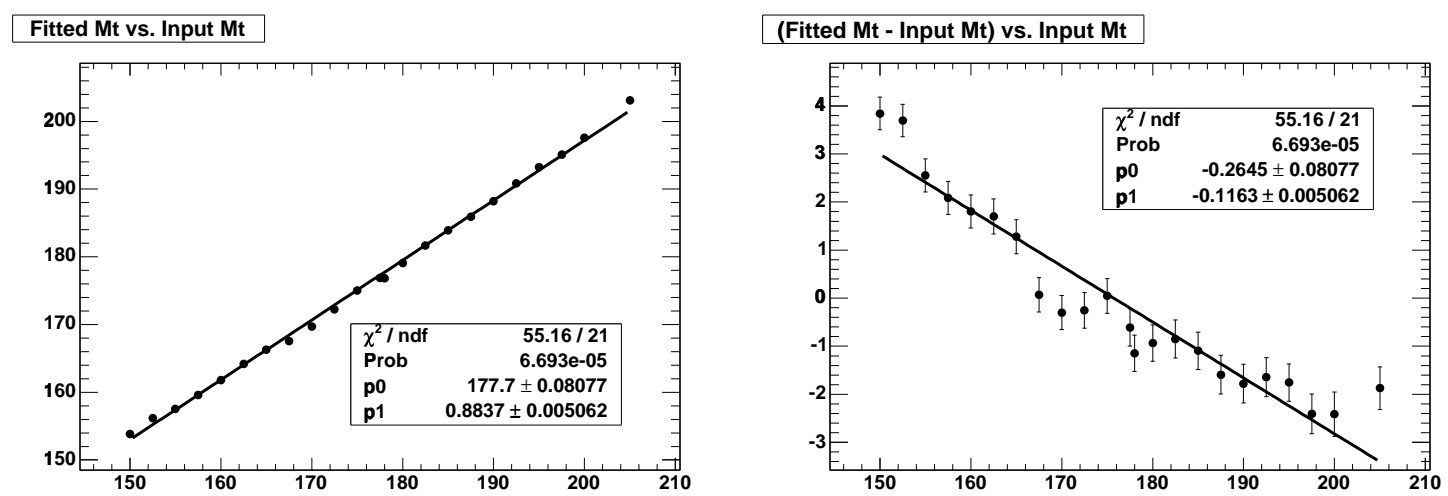

Figure 7.10: Fully simulated $t \bar{t}$ and background events analyzed with signal-only likelihood, pseudo-experiments with 35.94 Poisson-mean $t \bar{t}$ events and backgrounds as per Table 4.3. On the left is the usual linearity test, fitted $m_{t}$ vs. input $m_{t}$. On the right is the same information plotted as (fitted $m_{t}$ - input $m_{t}$ ) vs. input $m_{t}$. Both are fit to p0 $+\mathrm{p} 1 *$ (input $\left.m_{t}-178 \mathrm{GeV} / c^{2}\right)$. All axes are in $\mathrm{GeV} / c^{2}$. 


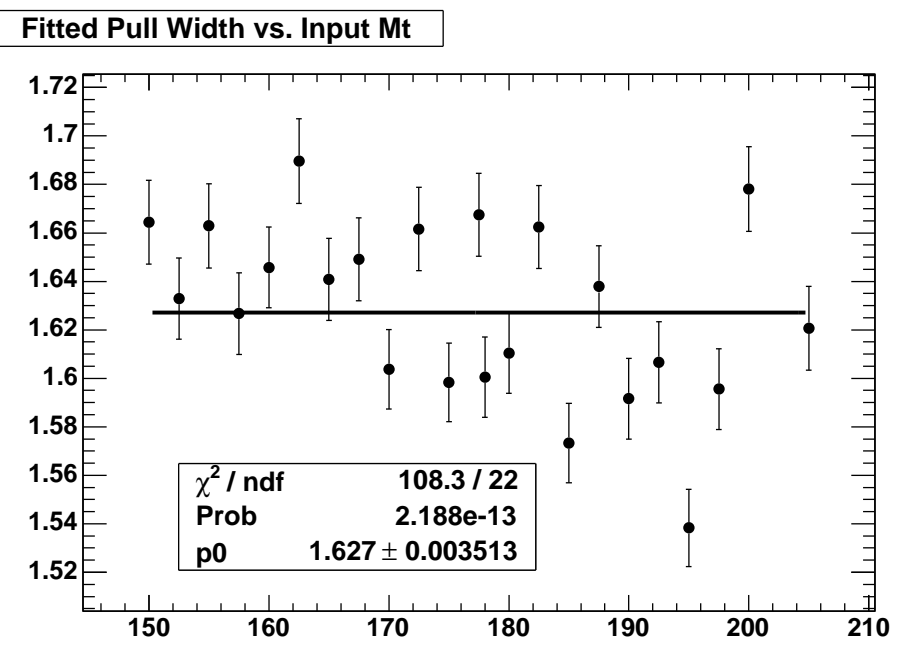

Figure 7.11: Fitted widths of pull distributions plotted vs input $m_{t}$. Fully simulated $t \bar{t}$ and background events analyzed with signal-only likelihood, 35.94 Poisson-mean $t \bar{t}$ events and backgrounds as per Table 4.3.

With the addition of unmodelled background our fitted $m_{t}$ are now considerably biased. For low input $m_{t}$ the background pulls the mass up, and likewise for high input $m_{t}$ it pulls it down, by as much as $3 \mathrm{GeV} / c^{2}$ in each case. Similarly, the pull widths are now 1.63, as compared with 1.46 for the case with only signal events. Our model now has the significant broken assumption that there are no background events, so some increase in the pull widths is expected. Likewise, the bias in $m_{t}$ due to background events is expected. As can be seen in Table 7.1 background events on there own tend to prefer $m_{t}$ values of $160-170$ $\mathrm{GeV} / c^{2}$. This tends not be a strong preference - the $P_{t \bar{t}}$ distributions for background events are not sharply peaked. But, it is enough to bias the $m_{t}$ measurement if the background is left unmodelled.

For comparison with other cases, the mean expected error at $178 \mathrm{GeV} / c^{2}$, taken from the fitted with of the $m_{t}$ distribution for that sample, is $4.10 \mathrm{GeV} / c^{2}$. So, the addition of of unmodelled background degrades our expected $m_{t}$ resolution by about $5 \%$ relative to the $3.91 \mathrm{GeV} / c^{2}$ expected with only signal events.

In order to avoid the bias on the measured $m_{t}$ and to try to avoid the degradation of our $m_{t}$ resolution, we chose to attempt to model the background using an additional matrix-element based probability. 


\section{Chapter 8}

\section{Background Probability}

Background processes are different from the signal process in an important way. While the signal process can be well described using a small number of diagrams (e.g. top quark pair production, where we even make the assumption of only including the $q \bar{q}$ production process), the background to these events with hard jets typically require more than an order of magnitude more diagrams to accurately describe the process. The QCD background is especially challenging, as it is not easy to model jets faking electrons.

As we will see, the $P_{\text {Back }}$ from the formalism of Chapter 5 will be defined in much the same way as the $P_{t \bar{t}}$ signal probability that we have explored in Chapter 7 . It is based upon a differential cross section, which in turn is based on a leading-order Standard Model matrix element.

We choose to extract the matrix-element calculation machinery from a well-known Monte Carlo event generator which employs effective approximations to estimate the matrix element. This has several advantages:

- It is straightforward to generate events from the same matrix element in order to test its evaluation

- The matrix element code is well known and has been extensively tested

- The authors of the code are able to provide assistance and technical knowledge.

As we will discuss, background probability machinery based on the ALPGEN [48] generator will be our default, while machinery based on the VECBOS [53] generator serves 
as a cross check. As we will see, the results from the two sets of machinery are highly correlated and either code could be used to similar effect in our final top mass measurement.

\subsection{ALPGEN}

There exists a wide variety of Monte Carlo event generators to choose from. Recently, generators such as COMPHEP and MADGRAPH have provided generation based directly on the explicit construction and evaluation of the Feynman diagrams. These generators offer a transparent and clean interface to the matrix element. However, in the case of a final state with more than a single jet, the number of diagrams quickly becomes unwieldy and the calculation very slow. For this reason, we have chosen to use the matrix element evaluation of the ALPGEN[48] generator as it offers the following advantages:

- The ALPHA algorithm avoids explicit generation and evaluation of every diagram

- Large, well-studied samples of generated events are available and form the basis of understanding of the experimentally collected data at CDF.

In the next sections, we discuss the prescription that we have developed for the evaluation of the matrix element by adapting a portion of the ALPGEN program.

\subsection{Interface to ALPGEN}

The matrix element calculated by ALPGEN is a function of the initial and final particles' spin, color, and flavor as well as momentum. In the generation of events, a single spin, color and flavor assignment is randomly generated. As this information is not available to us from detector measurements, we must perform a sum over all possible configurations.

As the number of final state particles increases, the number of spin and color configurations grows quickly; to rigorously sum over each of the possibilities would be impractical and, fortunately, unnecessary. It is possible to approximate the total sum with a statistical sampling. Figure 8.1 shows the distribution of $|M|^{2}$ in generated events using sums of a specific number of terms. So, the matrix element calculation converges fairly rapidly.

We sample spin and color configurations until the sum has converged, satisfying the criteria 

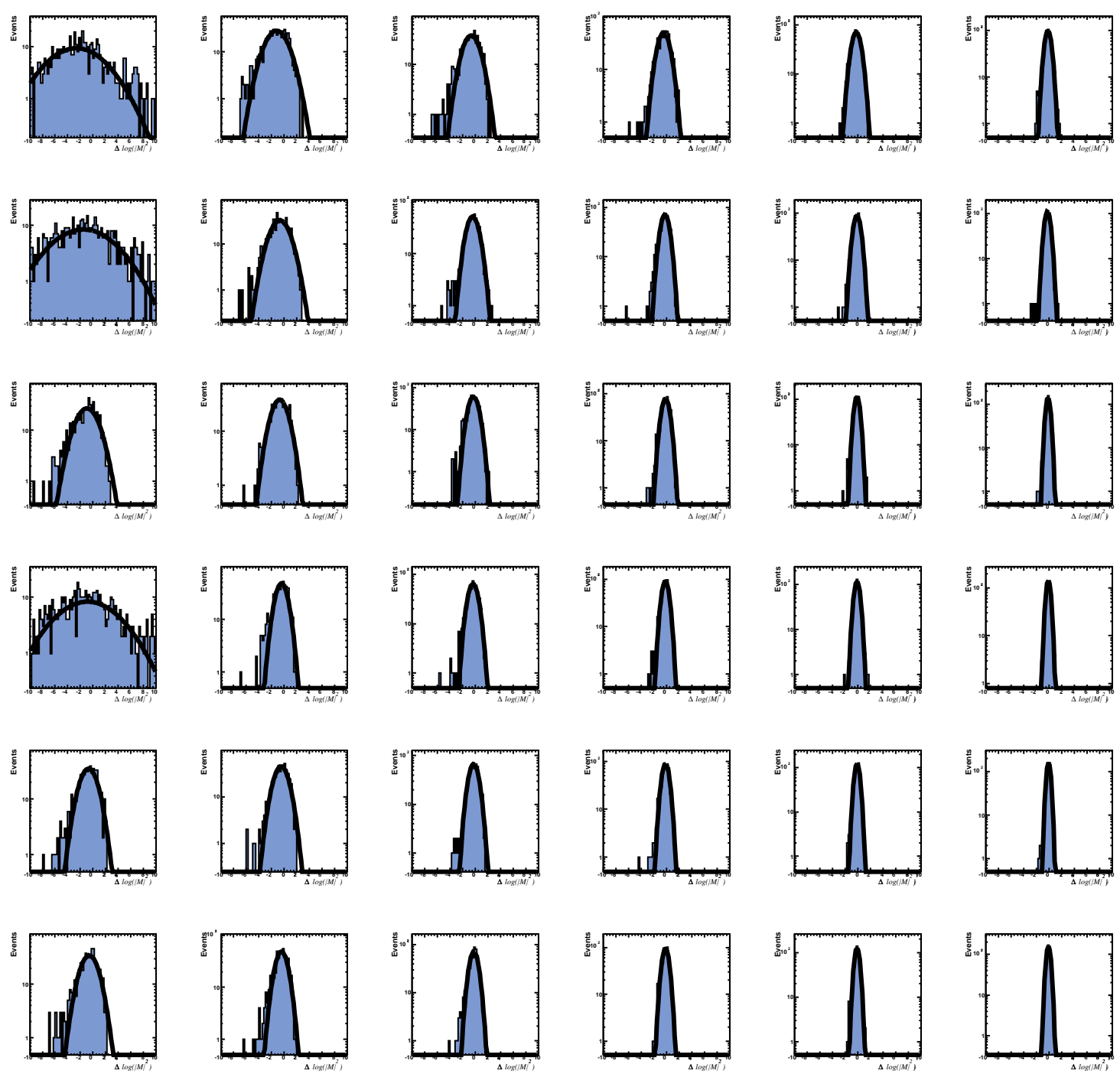

Figure 8.1: Variation in $\log \left(|M|^{2}\right)$ calculated with ALPGEN with an increasing number of terms in the spin and color sum. From the top, the number of spin terms sampled increases by powers of 2 from 1 to 32 . From the left, the number of color terms sampled increases by powers of 2 from 1 to 32 . Each plot represents many calculations of the matrix element for a particular particle configuration with a fixed number of samples in the spin and color sum. The $x$ axis of each plot is the variation in $\log \left(|M|^{2}\right)$ from the value found when using a large number of spin and color terms. The logarithmic $y$ axis is the number of calculations per bin. 


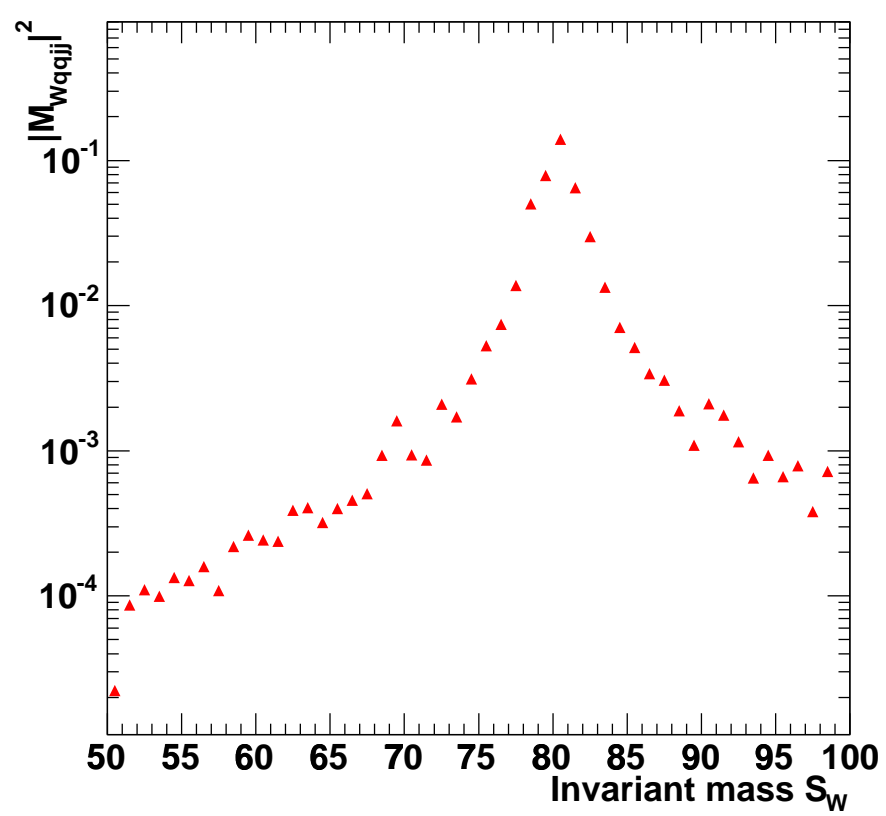

Figure 8.2: Variation of the squared ALPGEN matrix element for $p p \rightarrow W(\rightarrow l<n u)+$ $q q+j j$ as a function of the $l \nu$ invariant mass for a specific event.

$$
\frac{R M S\left(|M|^{2}\right)}{\operatorname{Mean}\left(|M|^{2}\right) \sqrt{N}}<\epsilon
$$

where $\epsilon$ is the convergence tolerance, set to $\epsilon=0.25$ for these calculations.

The calculation of the matrix element is well behaved; as an example, Figure 8.2 shows the variation of $\left|M_{W q q j j}\right|^{2}$ with the $l \nu$ invariant mass. It shows the expected strong peak at $M_{l \nu}=M_{W}$. The scatter from one mass point to the next gives a sense of the statistical uncertainty from the spin and color sampling.

\subsection{ALPGEN $W q q j j$ process}

As shown in Table 4.3 we expect backgrounds which can roughly be divided as $W+4 p$ mistags, $W+$ heavy flavor, QCD all-jet events, and small Electroweak backgrounds. As an approximation, we use matrix elements for just one of these processes. We calculate the background probability for the process 


$$
p \bar{p} \rightarrow W q q j j \rightarrow l \nu q q j j
$$

where the neutrino escapes without detection. In ALPGEN's terminology, $q$ here stands for a heavy flavor quark, either a $b$, or a $c$.

We expect that the probability based on this process will be reasonably effective, of course, for the $W+$ heavy flavor background, but also for the topologically very similar $W+4 p$ mistag background. We expect it to be less effective for QCD all-jet events and Electroweak di-boson events. And, perhaps single-top events will look more like $t \bar{t}$ than background through the lens of our $W+$ heavy flavor-based $P_{\text {Back }}$. This description turns out to be essentially true as we will describe later.

\subsubsection{Wqqjj: differential cross section}

Calculation of the differential cross-section for $W+4$ jet production requires integration of the matrix element over the phase space. In this case, the number of constraints from the measurement of the final state objects reduces the number of integrals to five.

We use the following notation: $q_{1}, q_{2}, q_{3}, q_{4}$ are the four-momenta of the final state partons which lead to the creation of jets, $L$ is the four-momenta of the final state lepton, $\nu$ the four-momentum of the final state neutrino and $p_{1}$ and $p_{2}$ are the four-momenta of the incoming partons.

The expression for the differential cross-section of this process is of the form:

$$
\begin{aligned}
d \sigma= & \sum_{a, b} \iint \frac{1}{(2 \pi)^{12}} \frac{1}{32} \frac{1}{E_{L} E_{\nu} E_{q_{1}} E_{q_{2}} E_{q_{3}} E_{q_{4}}} \\
& f\left(E_{q_{1}}, E_{j_{1}}\right) f\left(E_{q_{2}}, E_{j_{2}}\right) f\left(E_{q_{3}}, E_{j_{3}}\right) f\left(E_{q_{4}}, E_{j_{4}}\right) \\
& q_{1}^{R^{2}} \sin \theta_{q_{1}} q_{2}^{R^{2}} \sin \theta_{q_{2}} q_{3}^{R^{2}} \sin \theta_{q_{3}} q_{4}^{R^{2}} \sin \theta_{q_{4}} \\
& \delta\left(\phi_{q_{1}}-\phi_{j_{1}}\right) \delta\left(\theta_{q_{1}}-\theta_{j_{1}}\right) \delta\left(\phi_{q_{2}}-\phi_{j_{2}}\right) \delta\left(\theta_{q_{2}}-\theta_{j_{2}}\right) \\
& \delta\left(\phi_{q_{3}}-\phi_{j_{3}}\right) \delta\left(\theta_{q_{3}}-\theta_{j_{3}}\right) \delta\left(\phi_{q_{4}}-\phi_{j_{4}}\right) \delta\left(\theta_{q_{4}}-\theta_{j_{4}}\right) \\
& d q_{1}^{R} d \theta_{q_{1}} d \phi_{q_{1}} d q_{2}^{R} d \theta_{q_{2}} d \phi_{q_{2}} d q_{3}^{R} d \theta_{q_{3}} d \phi_{q_{3}} d q_{4}^{R} d \theta_{q_{4}} d \phi_{q_{4}} d^{3} \nu \\
& f_{\mathrm{PDF}}^{a}\left(\frac{p_{1}^{z}}{E_{\mathrm{beam}}}, Q^{2}\right) f_{\mathrm{PDF}}^{b}\left(\frac{p_{2}^{z}}{E_{\mathrm{beam}}}, Q^{2}\right) \frac{d p_{1}^{z} d p_{2}^{z}}{E_{\mathrm{beam}}^{2}} \\
& \delta^{4}\left(p_{1}+p_{2}-L_{1}-q_{1}-q_{2}-q_{3}-q_{4}\right) \frac{(2 \pi)^{4}}{4\left(p_{1}^{\mathrm{cm}} \sqrt{s}\right)}|\mathcal{M}|^{2} .
\end{aligned}
$$


In the above expression we have set the transverse components of incoming parton momenta to zero. We used this simplification also in the calculation of the signal probability and it is, in a sense, consistent with the leading order nature of our calculation. Of course, we expect this assumption to be violated in real collider data and also in realistic Monte Carlo.

So, with this collider approximation, $p_{1}^{0}=p_{1}^{z}$ and $p_{2}^{0}=p_{2}^{z}$. Integration over the $d p_{1}^{z} d p_{2}^{z}$ and $t$ and $z$ parts of the four-dimensional $\delta$-function result in the extra factor of $1 / 2$ and in the following substitution:

$$
\begin{gathered}
p_{1}^{z}=\frac{1}{2}\left(\left(L^{z}+q_{1}^{z}+q_{2}^{z}+q_{3}^{z}+q_{4}^{z}\right)+\left(L^{0}+q_{1}^{0}+q_{2}^{0}+q_{3}^{0}+q_{4}^{0}\right)\right) \\
p_{2}^{z}=\frac{1}{2}\left(\left(L^{z}+q_{1}^{z}+q_{2}^{z}+q_{3}^{z}+q_{4}^{z}\right)-\left(L^{0}+q_{1}^{0}+q_{2}^{0}+q_{3}^{0}+q_{4}^{0}\right)\right) .
\end{gathered}
$$

Evaluation of the integrals over $d^{3} q_{1} d^{3} q_{2}, d^{3} q_{3}, d^{3} q_{4}$ is performed in spherical coordinates, in order to take advantage of the angular $\delta$-functions. After the angular integrations, all $\phi_{q_{1,2,3,4}}$ and $\theta_{q_{1,2,3,4}}$ are replaced by the corresponding jet angles.

The entire expression for the differential cross-section is then:

$$
\begin{aligned}
& d \sigma=\sum_{a, b} \iint \frac{d q_{R_{1}} d q_{R_{2}} d q_{R_{3}} d q_{R_{4}} d \nu_{z}}{(2 \pi)^{12} 32 E_{L} E_{\nu} E_{q_{1}} E_{q_{2}} E_{q_{3}} E_{q_{4}}} \\
& f\left(E_{q_{1}}, E_{j_{1}}\right) f\left(E_{q_{2}}, E_{j_{2}}\right) f\left(E_{q_{3}}, E_{j_{3}}\right) f\left(E_{q_{4}}, E_{j_{4}}\right) q_{1}^{R^{2}} q_{2}^{R^{2}} q_{3}^{R^{2}} q_{4}^{R^{2}} \sin \theta_{j_{1}} \sin \theta_{j_{2}} \sin \theta_{j_{3}} \sin \theta_{j_{4}} \\
& f_{\mathrm{PDF}}^{a}\left(\frac{p_{1}^{z}}{E_{\mathrm{beam}}}, Q^{2}\right) f_{\mathrm{PDF}}^{b}\left(\frac{p_{2}^{z}}{E_{\mathrm{beam}}}, Q^{2}\right) \frac{(2 \pi)^{4}}{4\left(p_{1}^{\mathrm{cm}} \sqrt{s}\right)} \frac{|\mathcal{M}|^{2}}{2} .
\end{aligned}
$$

The flux factor $4\left(p_{1}^{\mathrm{cm}} \sqrt{s}\right)$ can be rewritten as $2\left|p_{1}^{z} p_{2}^{z}\right|$.

Converting to the language of Chapter $5, P_{b a c k}$ is $\frac{d \sigma}{\sigma}$ and the $P_{b a c k}$ term in Equa-

tion 5.8 is $\frac{d \sigma}{\sigma\langle\operatorname{Acc}(x)\rangle_{B a c k}}$. So, Equation 8.3 is our ALPGEN $P_{b a c k}$ apart from issues of normalization.

\subsubsection{Performance}

A test of the matrix element for $W q q j j$ using parton-level events is shown in Figure 8.3; the matrix element is able to separate $t \bar{t}$ events from $W q q j j$ events quite well. The separation at parton level is excellent, while applying a Gaussian resolution to the parton energies greatly diminishes the separation. One criteria for the performance of a background probability is simply that: a metric that returns a high value for a background event and a low value for a $t \bar{t}$ event. Then, the background event will be de-weighted in the 

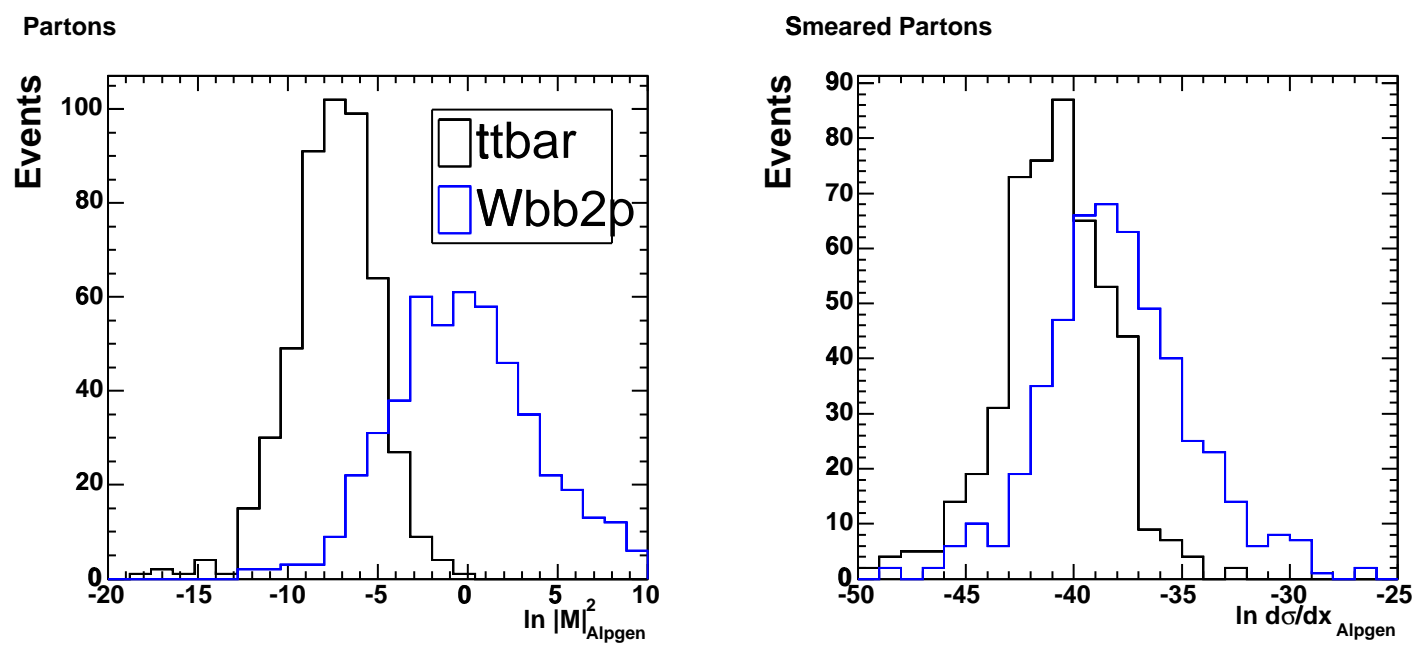

Figure 8.3: Left: evaluation of the squared matrix element for $p p \rightarrow W(\rightarrow l \nu)+q q+2$ jets for parton-level $t \bar{t}$ and $W+q q+2 p$ events. At parton level the separation between signal and background is quite good. Right: evaluation of the differential cross-section for $p p \rightarrow$ $W(\rightarrow l \nu)+q q+2 j e t s$ for parton-level events smeared with Gaussian transfer functions. Note that the separation between signal and background has already diminished.

overall likelihood and the well measured $t \bar{t}$ event, with a low background probability, will be relatively enhanced.

We also test the probability using fully simulated events. In Figure 8.4 we plot $P_{b a c k}$ for $t \bar{t}, W+4 p$, and $W b \bar{b}$ events. While the matrix element is that specifically of the $W q \bar{q}$ process we expect that the kinematics are very similar for all processes involving a $W$ and four jets $(W+4 p, W b \bar{b}, W c \bar{c}, W c)$. So, we expect $P_{b a c k}$ to do a good job describing these processes as well. And, in Figure 8.4 we see that the $P_{b a c k}$ distributions are indeed very similar for these two types of events. The separation seen here is comparable to that of the Gaussian smeared parton level events in Figure 8.3 suggesting that the energy resolution is the main factor in degrading the separation power.

In Figure 8.5 we suggest that, in a future version of this analysis, perhaps in a sample with no $b$ tags, a cut on $P_{b a c k}$ might productively be used as an event selection variable to enrich the sample. For example, we could retain $69 \%$ of $t \bar{t}$ events while removing $53 \%$ of $W+4 p$ events. 


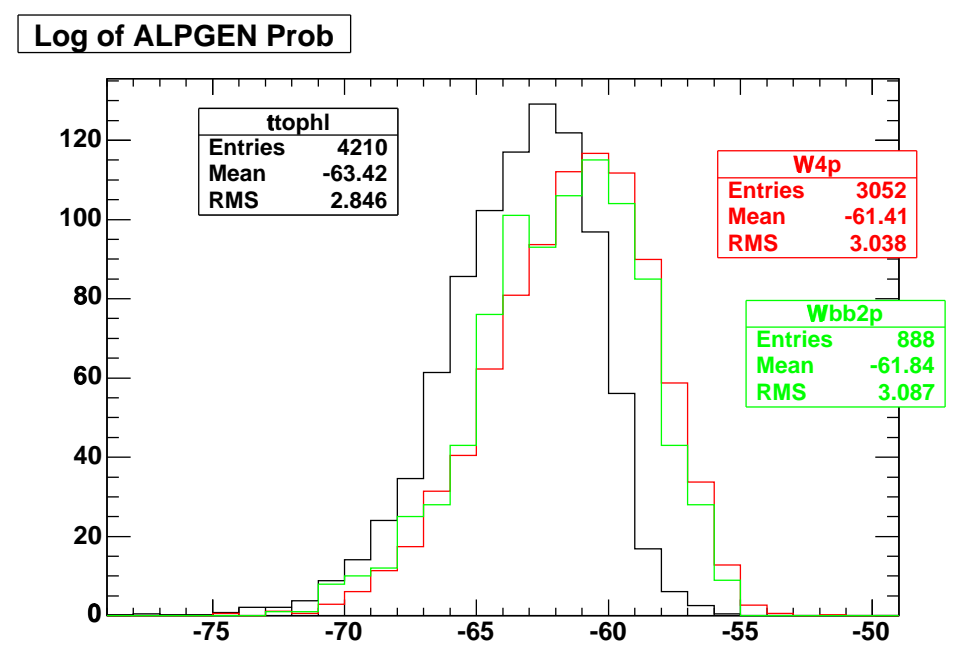

Figure 8.4: Log of the background differential cross section from ALPGEN for fully simulated HERWIG $178 \mathrm{GeV} / c^{2} t \bar{t}$ and ALPGEN+HERWIG $W+4 p$ and $W b \bar{b}$ samples. There is reasonably good separation between signal and background, and the $W+4 p$ and $W b \bar{b}$ samples behave in an extremely similar fashion.
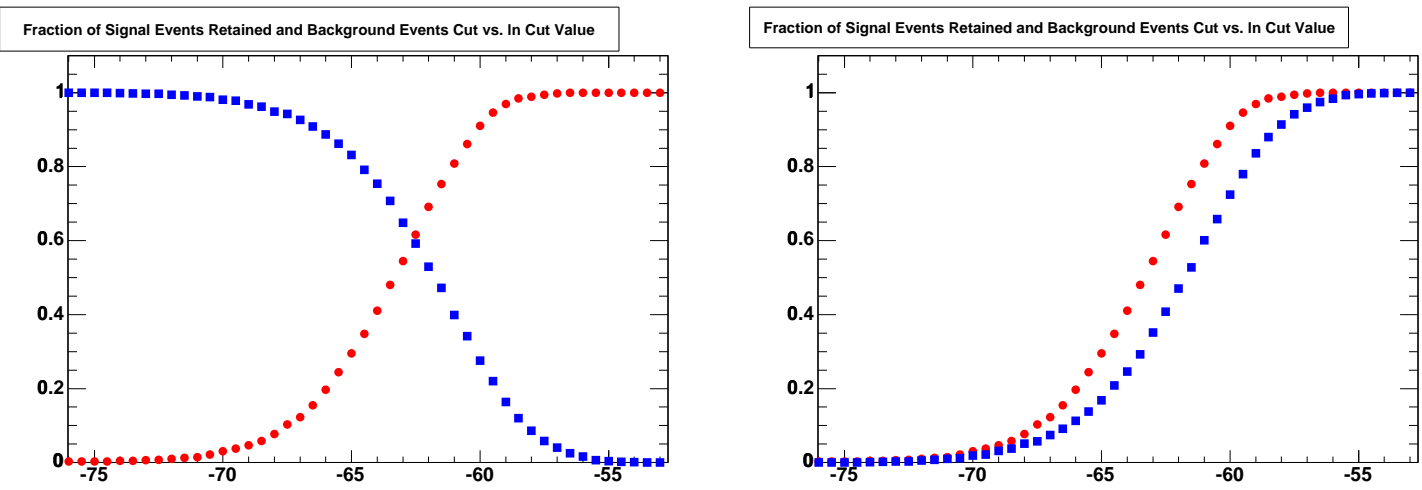

Figure 8.5: HERWIG $178 \mathrm{GeV} / c^{2} t \bar{t}$ (red circles) and ALPGEN+HERWIG $W+4 p$ (blue squares) processed with ALPGEN background probability. Left: We plot the fraction of $t \bar{t}$ events retained, and the fraction of $W+4 p$ events removed, as a function of $P_{b a c k}$ cut. We could keep $61.7 \%$ of $t \bar{t}$ while cutting $59 \%$ of $W+4 p$ or could keep $69 \%$ of $t \bar{t}$ while cutting $53 \%$ of $W+4 p$. Right: Or, we can rephrase that (looking at the plot on the right) and say that we can keep $61.7 \%$ of $t \bar{t}$ while keeping only $31 \%$ of the background. Here we plot the fraction of $t \bar{t}$ events retained and the fraction of $W+4 p$ events retained. 


\subsubsection{Normalization}

To use our ALPGEN $P_{\text {back }}$ in our overall likelihood (Equation 5.8) it needs to be normalized relative to $P_{t \bar{t}}$. Unlike $P_{t \bar{t}}$ there is no $m_{t}$-dependence to the $P_{b a c k}$ normalization, it is simply a number. One possibility for normalizing $P_{b a c k}$ would be to perform a direct integration, presumably by Monte Carlo, of $P_{b a c k}$ over all possible events. Unfortunately, this is a twenty dimensional integral and so computationally very difficult.

So, instead, we handle the relative normalization empirically. To do this we use Equation 5.8, our overall likelihood, in a somewhat different fashion than we will actually use on the data. Instead of fixing the signal fraction, $c_{1}$, according to our expectations, we perform a simultaneous two-dimensional fit for $m_{t}$ and $c_{1}$. In general, we do not expect this fit to return the actual fraction of signal events in the ensemble. Since the phase space of the $t \bar{t}$ and background events overlap, we expect, for example, that when we have only signal events, the fitted $c_{1}$ will still be less than 1.0. Likewise, for an ensemble of entirely background events the fitted $c_{1}$ will be greater than 0.0. So, as a compromise, we construct PE's of $50 \% t \bar{t}$ and $50 \%$ background events and tune the background normalization so that the fit returns $c_{1}=0.5$. The background events are selected from all of the ensembles that we expect our ALPGEN $P_{b a c k}$ to describe well, namely $W+4 p, W b \bar{b}, W c \bar{c}$, and $W c$. The number of events from each background is according to the fraction expected from Table 4.3. We also allow this empirical normalization to incorporate the background acceptance.

The empirical normalization we extract from this method is consistent with a preliminary, and fairly imprecise, normalization from direct 20-D Monte Carlo integration. And, it turns out that the actual 1-D fit that we will use on the data, where we fix $c_{1}$ in Equation 5.8, is not very sensitive to this relative normalization. Getting the relative normalization wrong is equivalent to using the wrong value of $c_{1}$ which, as we show in Section 11.7, does not greatly affect the measurement of $m_{t}$.

\subsection{W+Jets Probability using Leading Order VECBOS Ma- trix Element}

As a cross check to our ALPGEN based $P_{B a c k}$, we construct a probability density for $W+4$ jets events using a leading order matrix element from VECBOS. This leading order matrix element for $W$ production with $n$ associated jets $(n \leq 4)$ was calculated by $\mathrm{F}$. 
A. Berends, W. Giele, H. Kuijf, B. Tausk [53] and it is used to generate events as part of the VECBOS event generator. We pursued the VECBOS matrix element calculation because of its simple interface and the feature that it quickly and intrinsically performs a sum over all color and flavor possibilities. We use VECBOS version 3.0 with parameters tuned for CDF Run II.

In this section we describe the construction of our probability and our method of integration, and report on the performance of this VECBOS-based $P_{\text {Back }}$ for $W+$ jets and $t \bar{t}$ events. More details on the construction and testing of this probability can be found in $[75]$.

The $W+4$ jets probability density can be written as

$$
P_{\text {Back }}(\vec{x})=\sum_{p_{\nu}^{z}}^{2} \sum_{\text {comb }}^{24} \int\left|M_{W+4 j e t s}\right|^{2} \frac{f\left(\tilde{q}_{1}\right)}{\left|q_{1}\right|} \frac{f\left(\tilde{q}_{2}\right)}{\left|q_{2}\right|} \Phi_{6} d m_{W}^{2} \prod_{i=1}^{4} W_{j e t}\left(E_{i}^{y}, E_{i}^{x}\right) d p_{i},
$$

with six-particle phase space $\Phi_{6}$ as,

$$
\Phi_{6}=\frac{1}{2^{8}(2 \pi)^{14}} \frac{1}{E_{e}\left|E_{e} p_{z \nu}-p_{z e} E_{\nu}\right|} \prod_{i=1}^{4} \frac{E_{i}}{\left(p_{i}^{x}\right)^{2}},
$$

$M_{W+4 j e t s}$ as the $W+4$ jets matrix element from VECBOS, $f\left(\tilde{q}_{i}\right)$ the parton distribution function (PDF) to account for the unknown longitudinal momentum of colliding partons, and the transfer functions, $W(\vec{x}, \vec{y})$ to translate the measured jet energies to parton level energies as explained in Chapter 6.

The integration is reduced to 5 variables since we assume that the lepton momenta and jet angles are well measured. The parton energies and neutrino $p_{z}$ are not well measured, e.g. they have large experimental resolution, and therefore we need to integrate over them. The change in variables from the neutrino $p_{z}$ to $m_{W}^{2}$ results in kinematic solutions with two possibles values of $p_{z}$ for the neutrino, which we sum over. We also sum over all 24 possible jet-parton assignments.

Therefore, the background probability in Equation 8.5 includes four integrations over the large intervals of possible parton energies and one over a relatively narrow interval of the $W$ boson mass. The probability is sharply peaked around the $W$ pole mass so we need not consider such a large interval for this integral, in fact, we actually will approximate the $W$ Breit-Wigner as a delta function.

The integration process requires many evaluations of the matrix element and is very CPU intensive. We have developed a customized Monte Carlo method of integration 
utilizing our knowledge of the shape of the parton energy distributions from the transfer function. For a given event, we throw several random iterations of parton energies based on the measured jet energy from our transfer function. The $W$ boson mass is constrained to $80.4 \mathrm{GeV} / c^{2}$, and the transverse mass of the $e+\nu$ system is constrained to be less than this value. The reported background probability for an event is the average probability obtained from the Monte Carlo iterations.

Currently, we perform 400 iterations per event. This choice was determined by exploring how many iterations were necessary for reasonable convergence of the result, while considering also the amount of CPU used per event. The average CPU time for 400 iterations on a typical (e.g. $1 \mathrm{GHz}$ ) CPU is 13 minutes per event.

We evaluate the performance of our probability on fully simulated and reconstructed events passing the usual lepton+jets event selection criteria. That is, events that satisfy our full event selection with the exception of the $P_{t \bar{t}}$ cut. We compare the probability, $P_{\text {Back }}(\vec{x})$, using $t \bar{t}$ events from a HERWIG $t \bar{t}$ sample with $M_{t o p}=178 \mathrm{GeV} / c^{2}$ to the major backgrounds, $W+$ jets events and non- $W$ QCD. This comparison is shown in Figure 8.6. The figure contains simulated $W+4$ partons events from the electron channel, and the non- $W$ QCD events are the usual sample of non-isolated leptons in the side band of the signal region, from data. We observe a clear distinction between $t \bar{t}$ and the most significant background $(W+$ jets $)$ events. The result for non- $W$ QCD events is intermediate between $t \bar{t}$ and $W+$ jets, but there is still some .

In Figure 8.7 we see that fully simulated $W+4$ parton and $W b \bar{b}$ events behave in a very similar fashion when looking through the lens of our VECBOS background probability. And, there is in each case separation from $t \bar{t}$ events.

Figure 8.8 shows the discrimination efficiency of $t \bar{t}$ against $W+$ jets events as a function of a cut on the probability. For a selection cut on the logarithm of the probability of -56.2 we remove $68.3 \%$ of the $W+4$ jets events and $59.7 \%$ of the non- $W$ QCD events, while retaining $68.3 \%$ of $t \bar{t}$ events. So, the VECBOS background probability would actually be more effective than the ALPGEN calculation as a event selection variable, to discriminate signal from background. Presumably this is due not to the ALPGEN and VECBOS codes as we received them from their phenomenologist authors, but instead due to differences in the approximations we make in calculating the respective background probabilities based on these codes. And, as we will see, the difference disappears almost entirely when we use the two codes in the context of our full $m_{t}$ measurement. 


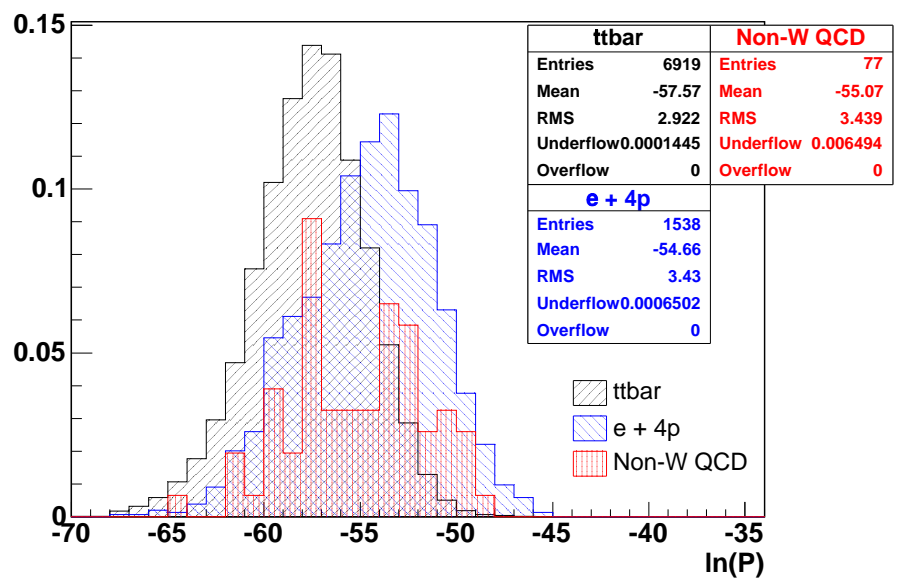

Figure 8.6: Distribution of VEBCOS probability density (Eq. 8.4) of fully simulated and reconstructed $W+$ jets, non- $W$ QCD and $t \bar{t}$ events $\left(M_{t o p}=178 \mathrm{GeV} / c^{2}\right)$. Note: the non- $W$ QCD events are scaled up by $50 \%$ for visibility.

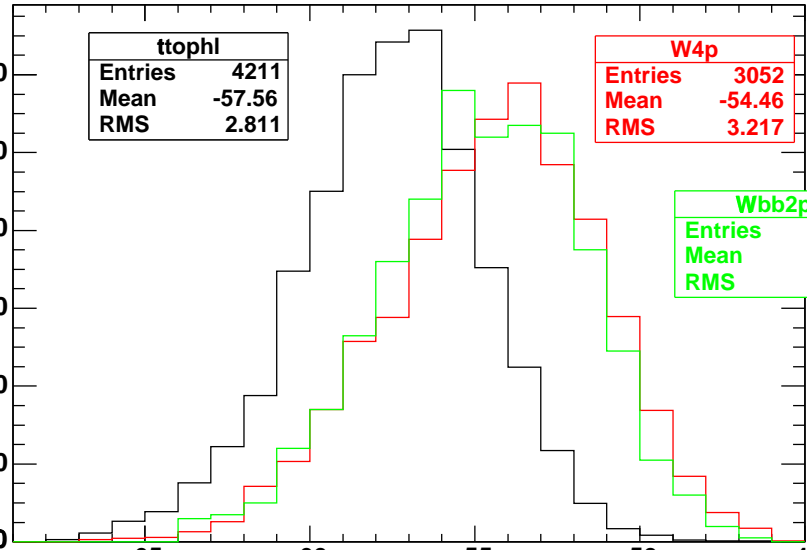




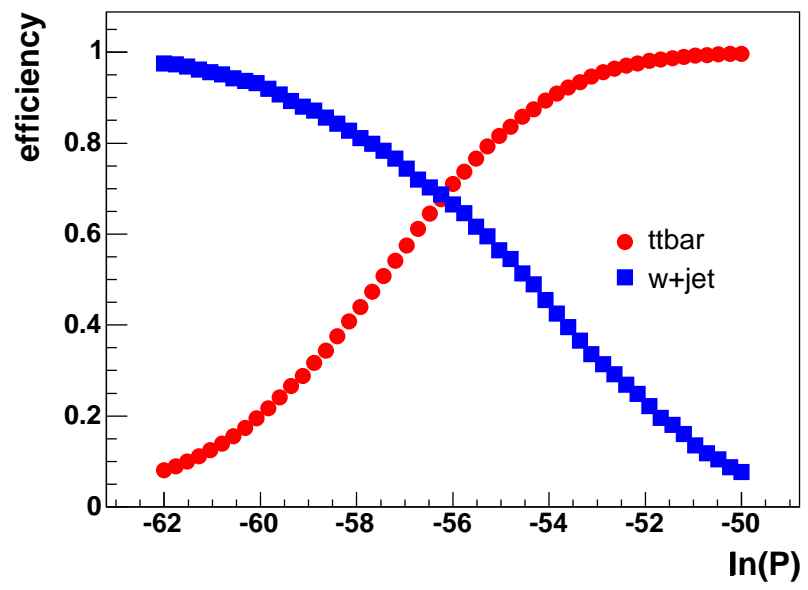

Figure 8.8: Discrimination between signal and background with the VEBCOS background probability. The samples are the same as in Fig 8.6 but the QCD background is not here included. We plot the fraction of $t \bar{t}$ events retained and the fraction of $W+$ jets events removed as a function of background probability cuts. So, this plot may be compared to the ALPGEN version which is the left-hand plot of Figure 8.5.

To use our VECBOS $P_{b a c k}$ in our overall likelihood, Equation 5.8 we need to ensure that it is normalized relative to $P_{t \bar{t}}$. We handle the normalization empirically, in the same fashion as we did for ALPGEN (see Section 8.3.3). The only difference is that we use fewer background samples for the VEBCOS tuning. We use $W b \bar{b}$ and $W+4 p$, weighted according to the expectations from Table 4.3.

\subsection{ALPGEN - VECBOS comparison}

In our current analysis VEBCOS serves as an important cross check to our default ALPGEN $P_{b a c k}$. It is clear from Figures 8.4 and 8.7 that if we were looking only for an event selection variable VECBOS would be a better choice. Perhaps because of differences in the integration routines, convergence criteria, and the like, the VEBCOS $P_{b a c k}$ does a better job of separating signal and background events through the one-dimensional lens of $P_{b a c k}$. However, this is only one indication of how the two $P_{b a c k}$ calculations would perform in the overall likelihood. As we will show below (in Section 9.1), the two codes give very similar results in a more realistic test of the overall $m_{t}$ resolution. For $m_{t}=178 \mathrm{GeV} / c^{2}$ pseudo-experiments constructed of 35.94 Poisson-mean $t \bar{t}$ and $8.67 W b \bar{b}$ and $W+4 p$ events 

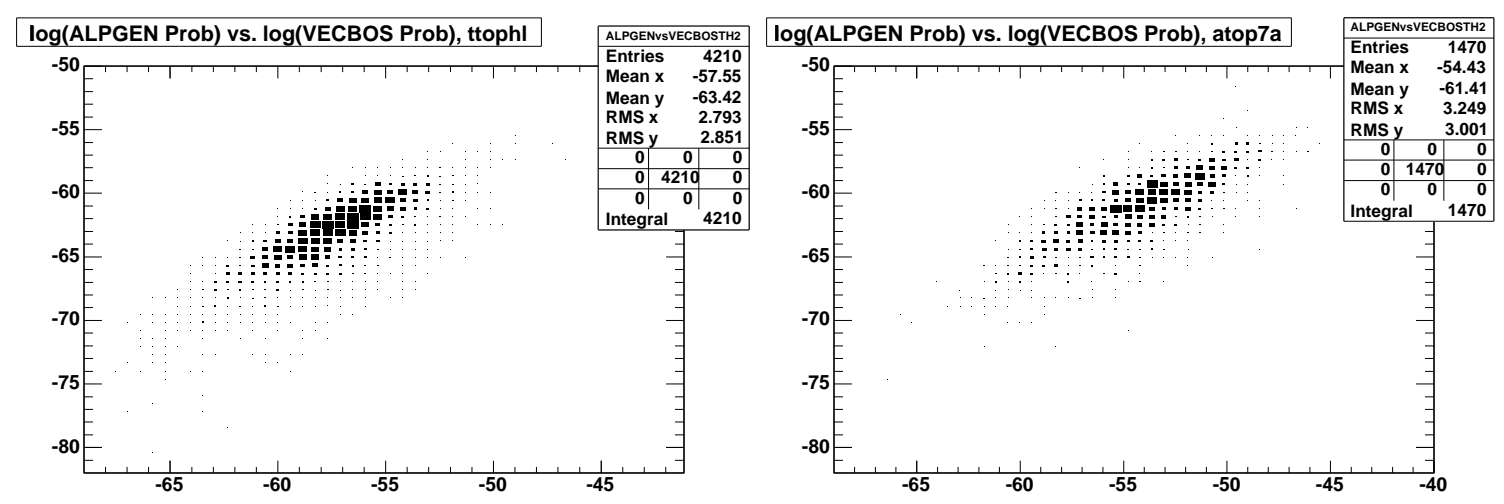

Figure 8.9: Log of ALPGEN probability vs. log of VECBOS probability for fully simulated $t \bar{t}$ (left) and $W+4$ parton, with the $W$ decaying to an electron (right), events.
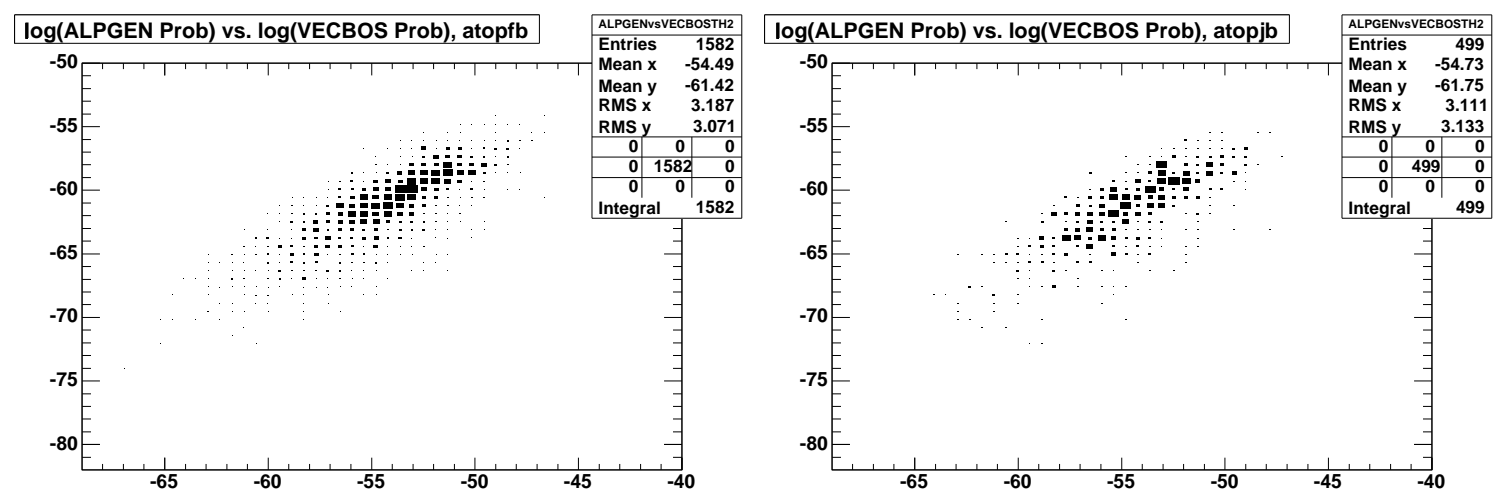

Figure 8.10: Log of ALPGEN probability vs. log of VECBOS probability for fully simulated $W+4$ parton events with the $W$ decaying to an electron (left) and $W b \bar{b}$ events with the $W$ decaying to a muon (right).

ALPGEN gives a mean expected error of $3.97 \mathrm{GeV} / c^{2}$ while VECBOS gives $3.98 \mathrm{GeV} / c^{2}$.

An interesting cross check of the robustness of each of the two, independent, $P_{b a c k}$ calculations is to compare their results on an event-by-event basis. Of course the two calculations are meant to encapsulate roughly the same physics, but they were developed independently and represent different choices at many points in the process of the calculation. In Figures 8.9-8.11 you can find plots of the unnormalized $P_{A L P G E N}$ vs. $P_{V E C B O S}$ for $t \bar{t}, W b \bar{b}$, and $W+4 p$ events. While there are outliers (and these are log-log plots, so the outliers are a significant distance from the bulk) there is a strong central region showing good event-by-event correlation between the two probabilities.

There are arguments to be made in favor of each of the ALPGEN and the VECBOS 


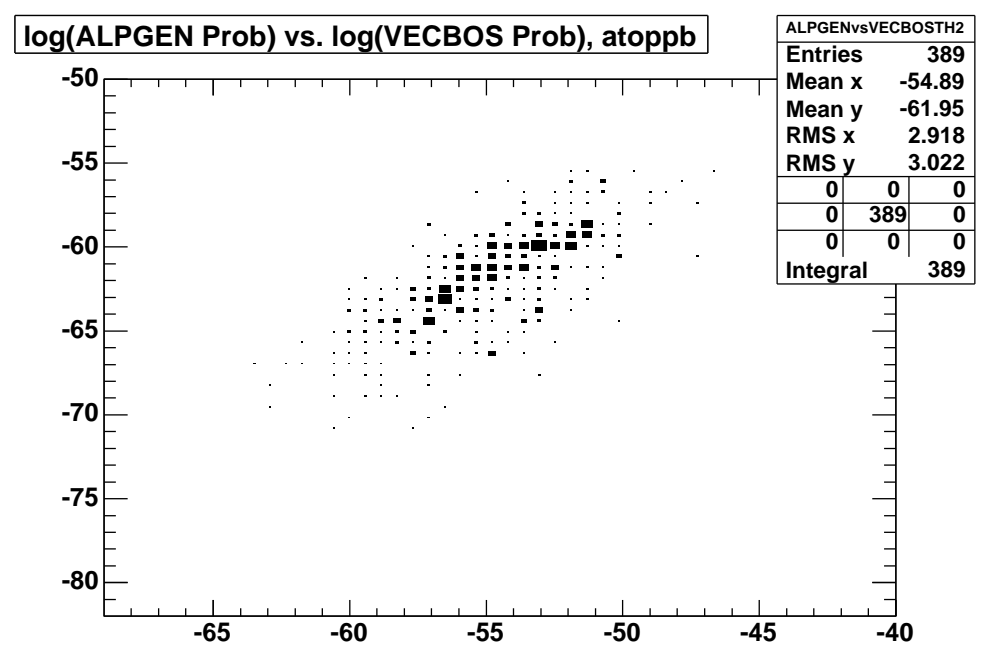

Figure 8.11: Log of ALPGEN probability vs. log of VECBOS probability for fully simulated $W b \bar{b}$ events where the $W$ decays to an electron.

$P_{\text {back }}$ calculations. The underlying ALPGEN code is actively supported by the authors and offers a wide variety of processes if we wish to consider adding additional background matrix elements. The VECBOS code has the advantage that the color, flavor, and spin sums are conducted automatically, and it seems to give better separation (in the 1-D $P_{b a c k}$ view) between signal and background events. Ultimately, empirical considerations lead us to choose ALPGEN as our default $P_{b a c k}$ calculation for this analysis. Our ALPGEN implementation runs significantly more quickly, and the development time-table for the ALPGEN implementation was such to allow a more timely $m_{t}$ analysis. It seems that, at least in their current incarnation, either code would give similar results in this $m_{t}$ analysis. 


\section{Chapter 9}

\section{Tests of Signal + Background Likelihood}

With the addition of $P_{b a c k}$ we can analyze pseudo-experiments using the full likelihood of Equation 5.8. We perform a one-dimensional fit to extract $m_{t}$, fixing $c_{1}$ at the expected value. We continue to characterize two different types of pseudo-experiments, those based upon a $6.1 \mathrm{pb} t \bar{t}$ cross section and those based on the 63 events we observe in data. Along the way we can make comparisons between the ALPGEN and VECBOS-based background probabilities, and explore the effects of modelled and unmodelled backgrounds. We also extract a mapping function to correct raw measured $m_{t}$ values and errors for both the $6.1 \mathrm{pb}$ and 63 event cases. This mapping function, or procedure, is necessary to extract unbiased results, with accurately estimated errors, from Monte Carlo pseudo-experiments, or from real collision data.

\subsection{Pseudo-Experiments for $\sigma_{t \bar{t}}=6.1 \mathrm{pb}$}

First, we handle the $6.1 \mathrm{pb}$ case, with 35.94 Poisson-mean $t \bar{t}$ events and backgrounds according to Table 4.3. Here we fix the signal fraction at $c_{1}=0.8056$, according to our expectations. The linearity plot can be found in Figure 9.1, the residuals (fitted $m_{t}$ - input $\left.m_{t}\right)$ in Figure 9.2, and the widths of pull distributions in Figure 9.3. In all cases we consider a range of $m_{t}$ values. Since we are now modelling the background we expect improved performance over the case of $t \bar{t}$ and background events in Chapter 7 .

The linearity is in very good agreement with slope 1.0. For low values of $m_{t}$ we 


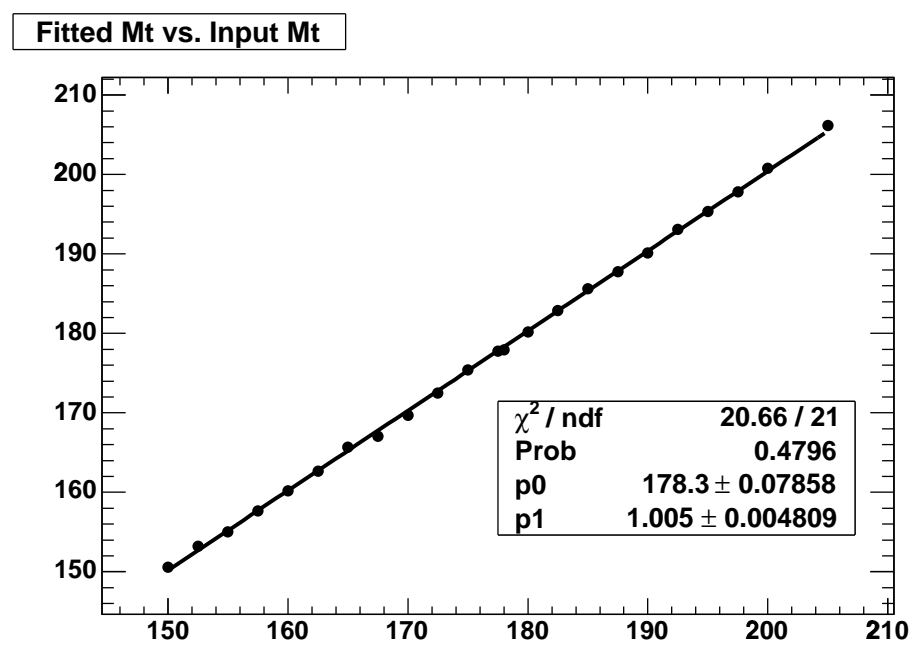

Figure 9.1: Fully simulated $t \bar{t}$ and background events analyzed with the full signal + background likelihood of Equation 5.8. Pseudo-experiments are of the $6.1 \mathrm{pb}$ type. The usual linearity test, fitted $m_{t}$ vs. input $m_{t}$. The fit is to $\mathrm{p} 0+\mathrm{p} 1 *$ (input $m_{t}-178 \mathrm{GeV} / c^{2}$ ). All axes are in $\mathrm{GeV} / c^{2}$.
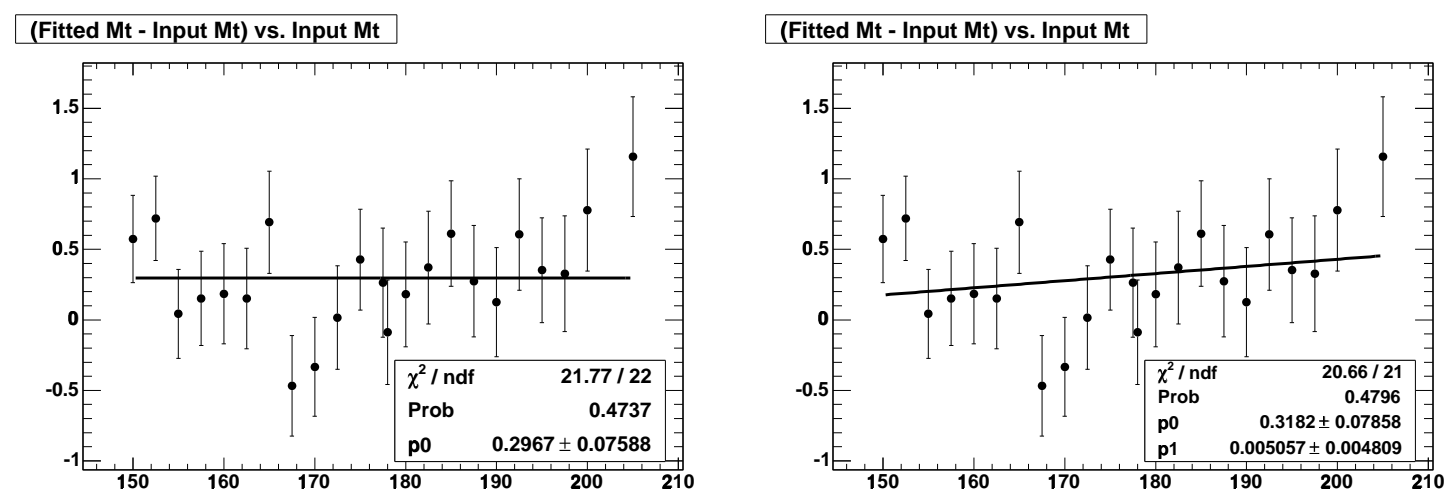

Figure 9.2: Fully simulated $t \bar{t}$ and background events analyzed with our full likelihood. Both plots are of (fitted $m_{t}$ - input $m_{t}$ ) vs. input $m_{t}$, the $m_{t}$ residuals. On the left is the flat fit that we use for our mapping function. On the right is an alternate linear fit to p0 + p1 * (input $\left.m_{t}-178\right)$. From the plot on the left we extract the mapping function that we use for $6.1 \mathrm{pb}$ PE's, a constant correction of $-0.2967 \mathrm{GeV} / c^{2}$. 


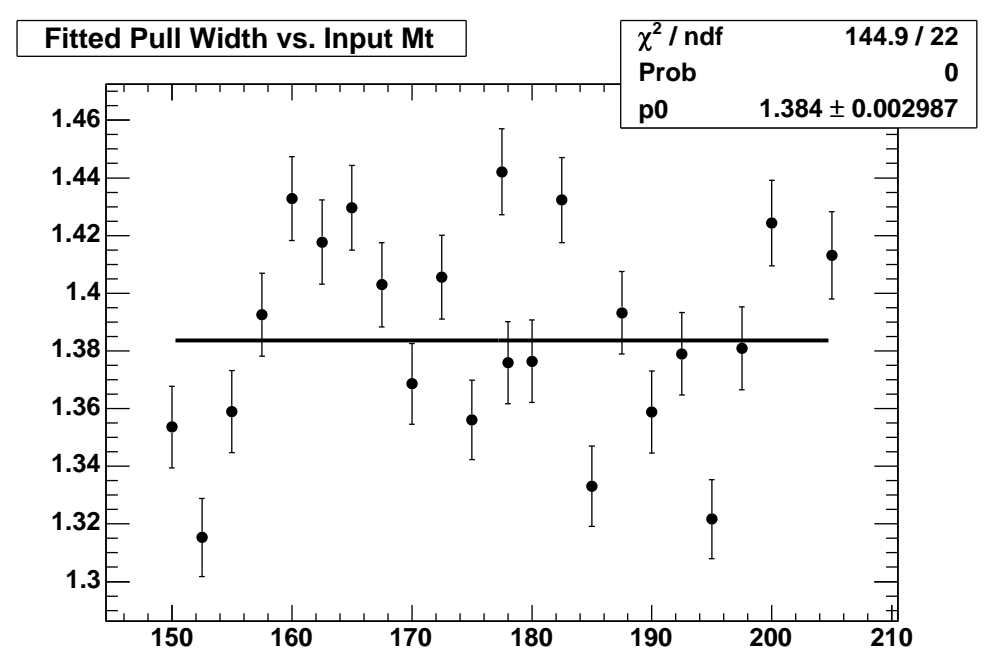

Figure 9.3: Fully simulated $t \bar{t}$ and background events analyzed with our full likelihood. Fitted widths of pulls vs. $m_{t}$ for resampled $6.1 \mathrm{pb}$ pseudo-experiments. From this plot we extract the smearing factor of 1.384 by which we multiply our estimated error, as part of our mapping function, for $6.1 \mathrm{pb}$ pseudo-experiments.

had seen a tendency to be pulled high, but those events which preferred higher top masses have apparently been ameliorated by the background probability. So, adding the background probability greatly improves the linearity of the $m_{t}$ measurement. To address any residual bias we extract a mapping function from Figure 9.2. We consider two possibilities, a correction independent of $m_{t}$ and a correction linear in $m_{t}$. The fit to a linear function has a slightly higher probability, but the fit to a flat function is entirely reasonable. So, as a mapping function for our 6.1-pb style pseudo-experiments we choose a constant function, $-0.2967 \mathrm{GeV} / c^{2}$. To make an unbiased measurement of $m_{t}$ we need to apply this small correction to the raw, naively estimated $m_{t}$, values that maximize the likelihood.

The pull widths, at 1.384, are significantly reduced from the case with unmodelled background (1.68), and are better even than the case with signal events and a signalonly likelihood (1.46). The background probability gives an outlet to $t \bar{t}$ events which are either poorly measured or otherwise inconsistent with our leading-order $t \bar{t}$ hypothesis and so improves the approximation even of our modelling of the signal events. As a part of our mapping procedure, we will multiply our naively estimated error by this factor of 1.384 , in order to accurately estimate our error on $m_{t}$.

The mean expected error at $178 \mathrm{GeV} / c^{2}$, taken from the fitted width of the $m_{t}$ distribution for that sample, is $4.03 \mathrm{GeV} / c^{2}$. So, relative to the case with unmodelled 
background $\left(4.10 \mathrm{GeV} / c^{2}\right)$ our resolution improves by about $2 \%$. But, relative to the 3.91 $\mathrm{GeV} / c^{2}$ mean expected error from the signal-only case we have still lost $3 \%$ resolution. We can compare also to a case where we include only those backgrounds which we expect to be well handled by our $W+$ heavy flavor background model. So, we also construct pseudoexperiments using the same number of background events as usual, but with only $W b \bar{b}$ and $W+4 p$ events. Thise case with only "well-modelled" backgrounds has a $m_{t}$ distribution with fitted width of $3.97 \mathrm{GeV} / c^{2}$. Some information is lost with the inclusion of even these "well-modelled" backgrounds - our parton-level background model with transfer functions does not accurately model fully simulated Monte Carlo (or real data) background events. And there is some irreducible component of the background, the phase space of background and $t \bar{t}$ events is expected to actually overlap.

Thus, including a matrix-element based background probability does improve our resolution. If we include background events in our pseudo-experiments and do not model them at all (or model them only with a mapping function based on Figure 7.10) our $m_{t}$ resolution degrades by about 5\%. By including the matrix-element based background probability we are able to recover about half of this loss.

Next, we rerun these same pseudo-experiments with the mapping function of Figures 9.2 and 9.3 applied, as a consistency check. That is, we shift the raw $m_{t}$ values downward by $0.2967 \mathrm{GeV} / \mathrm{c}^{2}$ and multiply the raw error estimate by 1.384 . The resulting linearity plot and residuals may be found in Figure 9.4 and look very good, the linearity consistent with unity and the residuals consistent with zero.

The calibrated pull widths may be found in Figure 9.5. The left hand plot there contains the errors as estimated with the methodology of Section 7.4. As we mentioned in that Section, it seems that we tend to underestimate the errors on pull widths when we use this resampling methodology. So, in the right hand plot we empirically inflate the errors until the $\chi^{2}$ probability is reasonable. Using this procedure, we get a better sense of what the error on the fitted mean of the pull widths is, and see that after our mapping function we are within about $1 \sigma$ of unity.

I should note that this inflation of errors due to resampling is a separate issue from that of the inflation of errors due to the non-unit $m_{t}$ pull widths. The former is an issue of central importance in the estimation of the experimental uncertainty on $m_{t}$. It is important to understand the pull width inflation factor, and we will explore this in greater detail in Section 12.1. The resampling inflation factor, as used for the right hand plot in Figure 9.5, 

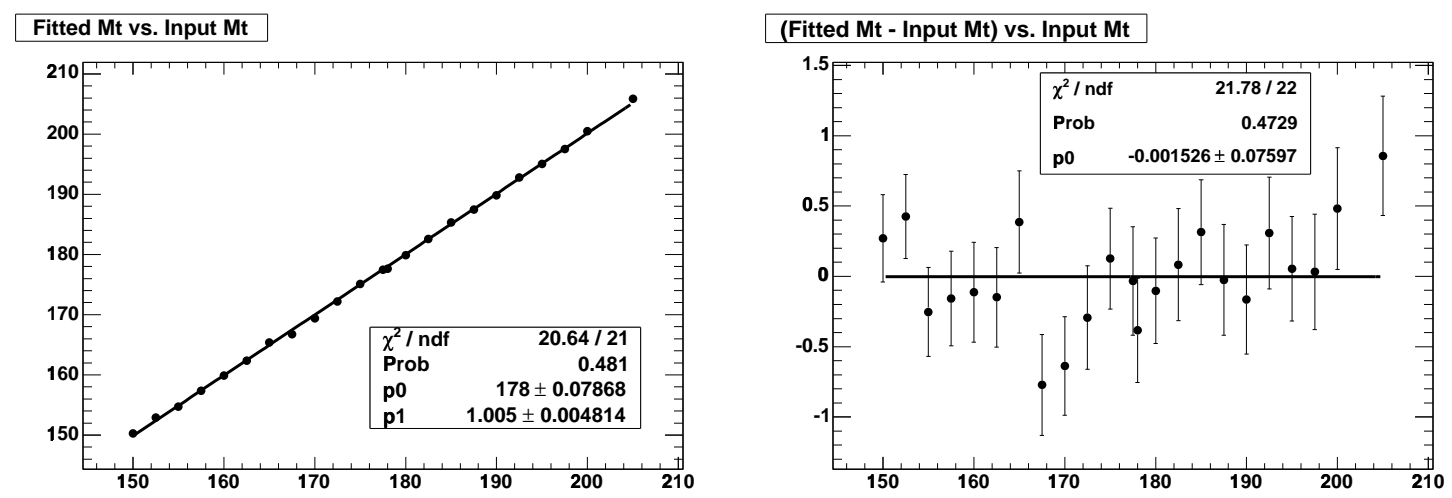

Figure 9.4: Fully simulated $t \bar{t}$ and background events analyzed with our full likelihood and mapping function. Pseudo-experiments of the $6.1 \mathrm{pb}$ style, 35.94 Poisson-mean signal events and backgrounds as per Table 4.3. On the left is the usual linearity test, fitted $m_{t}$ vs. input $m_{t}$. On the right is the same information plotted as (fitted $m_{t}$ - input $m_{t}$ ) vs. input $m_{t}$. The left-hand plot is fit to $\mathrm{p} 0+\mathrm{p} 1 *$ (input $m_{t}-178$ ) and the right-hand plot to a constant.
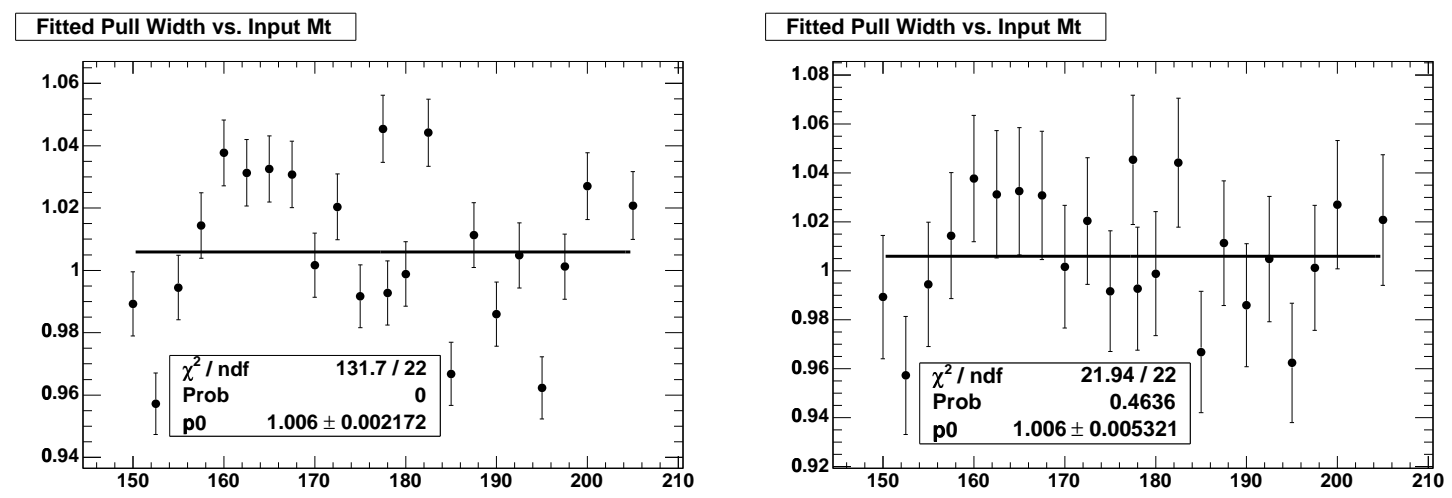

Figure 9.5: Fully simulated $t \bar{t}$ and background events analyzed with our full likelihood and mapping function. Pseudo-experiments of the $6.1 \mathrm{pb}$ style, 35.94 Poisson-mean signal events and backgrounds as per Table 4.3. Fitted widths of pulls from resampled pseudoexperiments. The left-hand plot has errors extracted directly from the fit to resampled pseudo-experiments. The right-hand plot has errors inflated by an empirical factor, as we seem to be underestimating the errors for quantities extracted from resampled pseudoexperiments. 


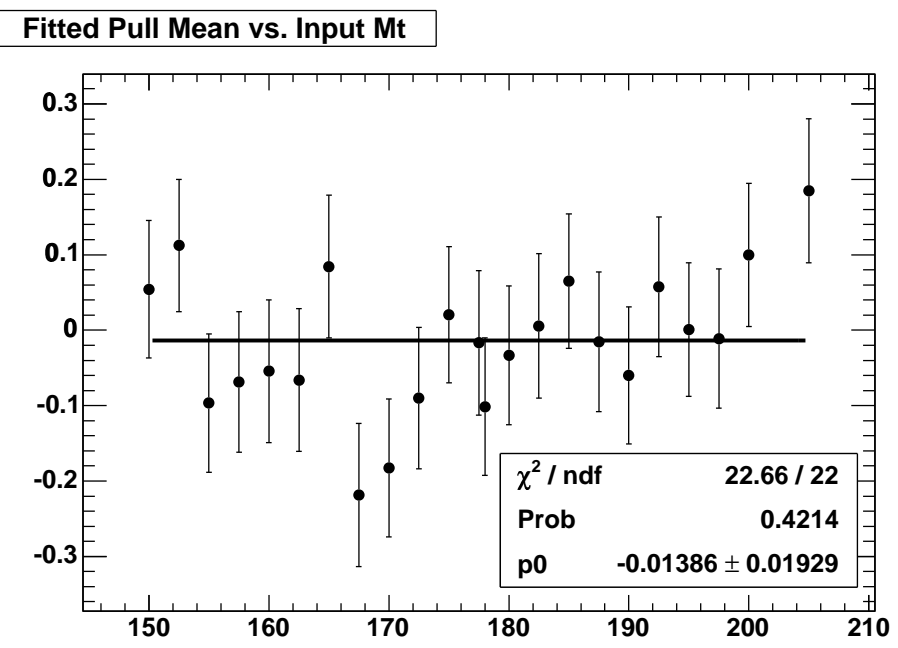

Figure 9.6: Fully simulated $t \bar{t}$ and background events analyzed with our full likelihood and mapping function. Pseudo-experiments of the $6.1 \mathrm{pb}$ style, 35.94 Poisson-mean signal events and backgrounds as per Table 4.3. Fitted means of pull distributions from resampled pseudo-experiments.

affects only those quantities that we extract from resampled pseudo-experiments. This includes especially the error on the width of pull distributions, but not the width of the pull distributions itself. So, it affects what conclusions we can draw about the uniformity of pull widths as a function of mass, but in no way directly enters into our final $m_{t}$ measurement or its errors. We know that resampled pseudo-experiments do not contain as much information as unresampled pseudo-experiments: they are by definition correlated. This resampling inflation factor suggests that we are perhaps resampling the pseudo-experiments too heavily, and introducing a non-trivial correlation. But, the only effect is to underestimate the statistical error on quantities like the width of pull distributions ${ }^{1}$.

For completeness, we also plot the fitted means of the pull distributions in Figure 9.6.

We also construct pseudo-experiments to compare the $m_{t}$ resolution of our analysis using our default ALPGEN-based $P_{b a c k}$ and the alternate VECBOS-based $P_{b a c k}$. Since we have a smaller number of VECBOS-processed samples available we use only the $W b \bar{b}$ and $W+4 p$ backgrounds for this comparison. Using 35.94 Poisson mean $t \bar{t}$ events and 8.67

\footnotetext{
${ }^{1}$ Since the resampling inflation factor is of relatively little consequence, we may not always note when it is applied. In general, when the $\chi^{2}$ probability of a plot of pull widths as a function of $m_{t}$ is reasonable, the inflation factor has been applied. Note that the plots of measured $m_{t}$ vs. input $m_{t}$ naturally have reasonable $\chi^{2}$ probabilities - they are not affected by resampling considerations.
} 
$W b \bar{b}$ and $W+4 p$ events weighted according to Table 4.3 we estimate the $m_{t}$ resolution by considering the fitted width of $m_{t}$ results from pseudo-experiments. We find that ALPGEN gives a resolution of $3.97 \mathrm{GeV} / c^{2}$ and VECBOS $3.98 \mathrm{GeV} / c^{2}$. So, the two give very similar results. We have already compared this $3.97 \mathrm{GeV} / c^{2}$ result to the $4.03 \mathrm{GeV} / c^{2}$ resolution for ALPGEN when including all seven expected background contributions. This suggests that the effect of poorly modelled background contributions (i.e. QCD, single top, EW) is at about the $1.5 \%$ level.

\subsection{Pseudo-Experiments for 63-Event Samples}

Now, we consider the other type of pseudo-experiment, the type from which we will extract a mapping function to use on the actual collision data and to estimate our systematics. We will continue to Poisson fluctuate our background contributions about the means from Table 4.3, now again using all seven expected background contributions. But, since we have 63 events in data, we will use 54.33 Poisson-mean $t \bar{t}$ events. And, here we fix the signal fraction as $c_{1}=0.8624$. For these pseudo-experiments the linearity plot can be found in Figure 9.7 and the residuals in Figure 9.8. In Figure 9.8 we present a flat fit as well as a linear fit. Since the $\chi^{2}$ probability for the flat fit is quite reasonable, that is, the distribution is consistent with being flat, we will use a constant mapping function of $-0.2755 \mathrm{GeV} / c^{2}$. However, since the linear fit has a higher $\chi^{2}$ probability we will consider the difference between the two as a systematic error (see Section 11.10).

The widths of the pull distributions may be found in Figure 9.9. From this plot we extract the factor of 1.356 by which we will multiply our error for 63-event pseudoexperiments as part of our mapping function, or procedure. This is a bit smaller than the 1.384 factor from the $6.1 \mathrm{pb}$ case, which is consistent with a general trend we have seen toward smaller pull widths with larger PE's. Presumably this is because, for smaller PE's, our assumption of a Gaussian likelihood distribution (and hence a parabolic log $L$ distribution) is not as good.

Then, as a consistency check, we also construct similar pseudo-experiments with our 63-event mapping function applied. Plots from these ensembles may be found in Figures 9.10, 9.11, and 9.12. These ensembles have had the $-0.2755 \mathrm{GeV} / c^{2} m_{t}$ correction and the multiplicative 1.356 error inflation factor applies. The pull widths, after mapping, are consistent with unity and the pull means with zero. So, after a modest correction to the 


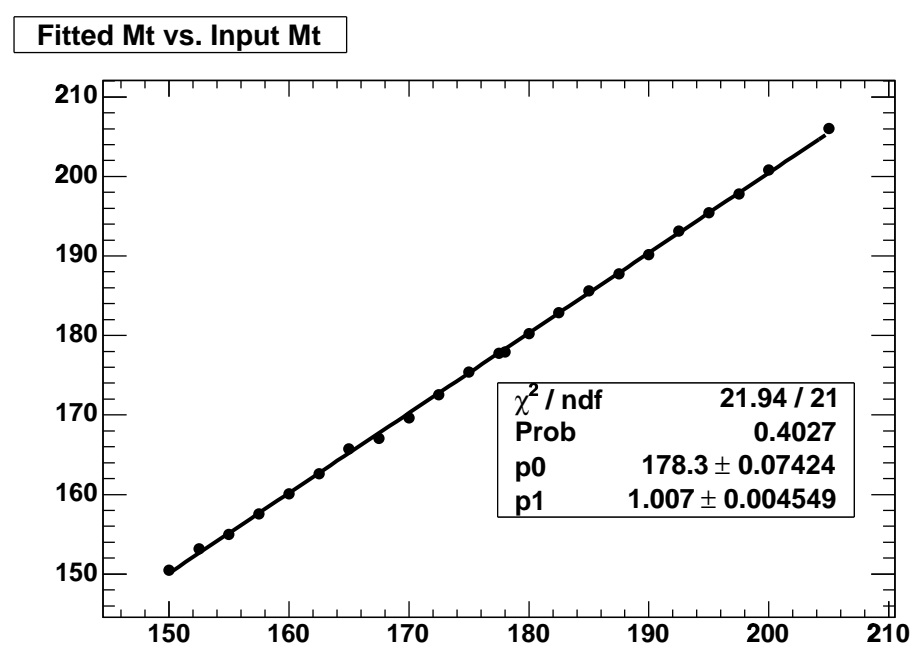

Figure 9.7: Fully simulated $t \bar{t}$ and background events analyzed with the full signal + background likelihood of Equation 5.8. Pseudo-experiments are of the 63-event type. The usual linearity test, fitted $m_{t}$ vs. input $m_{t}$. The fit is to $\mathrm{p} 0+\mathrm{p} 1 *$ (input $m_{t}-178 \mathrm{GeV} / c^{2}$ ). All axes are in $\mathrm{GeV} / c^{2}$.
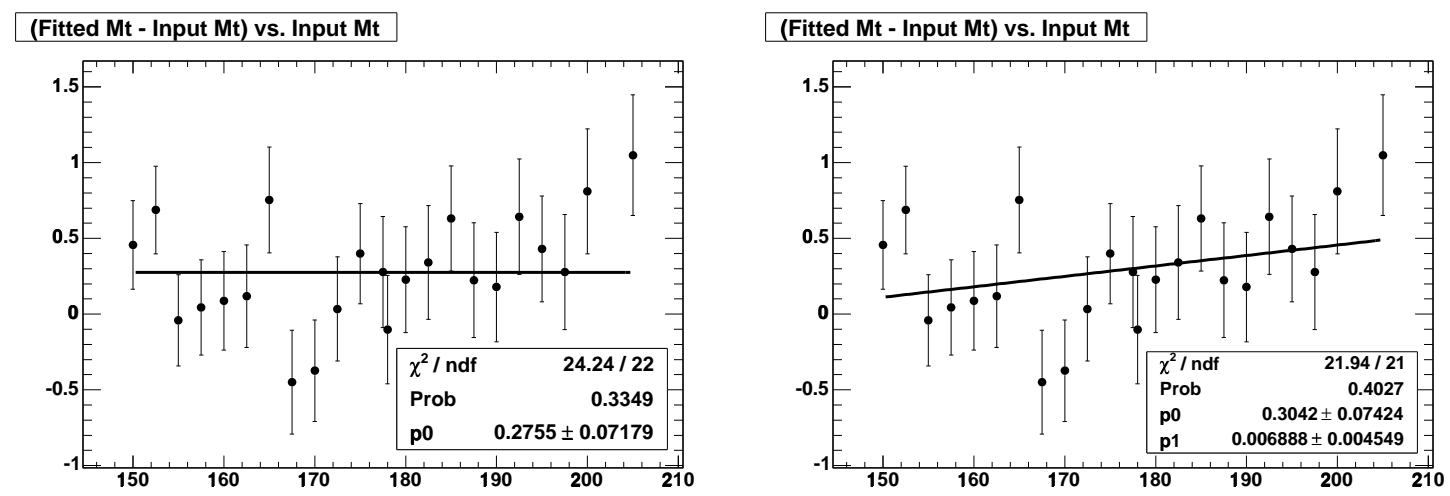

Figure 9.8: Fully simulated $t \bar{t}$ and background events analyzed with our full likelihood. Both plots are of (fitted $m_{t}$ - input $m_{t}$ ) vs. input $m_{t}$, the $m_{t}$ residuals. On the left is the flat fit that we use for our mapping function. On the right is an alternate linear fit to p0 + p1 * (input $\left.m_{t}-178\right)$. From the plot on the left we extract the mapping function that we use for 63 -event PE's, a constant correction of $-0.2755 \mathrm{GeV} / c^{2}$. 


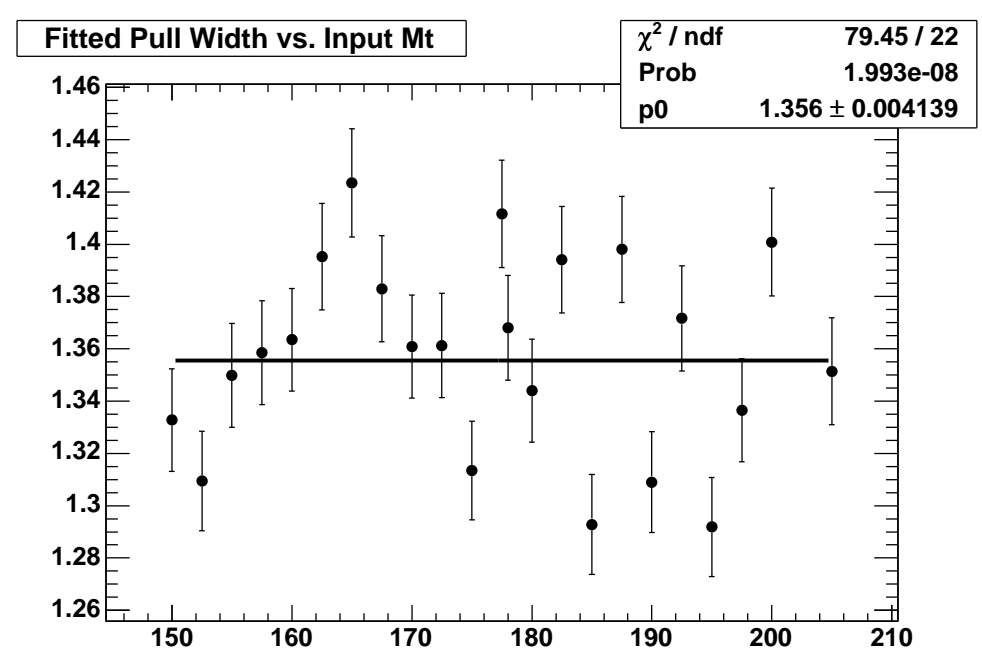

Figure 9.9: Fully simulated $t \bar{t}$ and background events analyzed with our full likelihood. Fitted widths of pulls vs. $m_{t}$ for resampled 63 -event pseudo-experiments. From this plot we extract the smearing factor of 1.356 by which we multiply our estimated error, as part of our mapping function, for 63-event pseudo-experiments.

measured $m_{t}$ and a more significant correction to the naively estimated error we have an unbiased measurement of $m_{t}$ with an accurately estimated error.

For this case, we also plot the mean of the estimated error distribution, as a function of $m_{t}$, in Figure 9.13. 

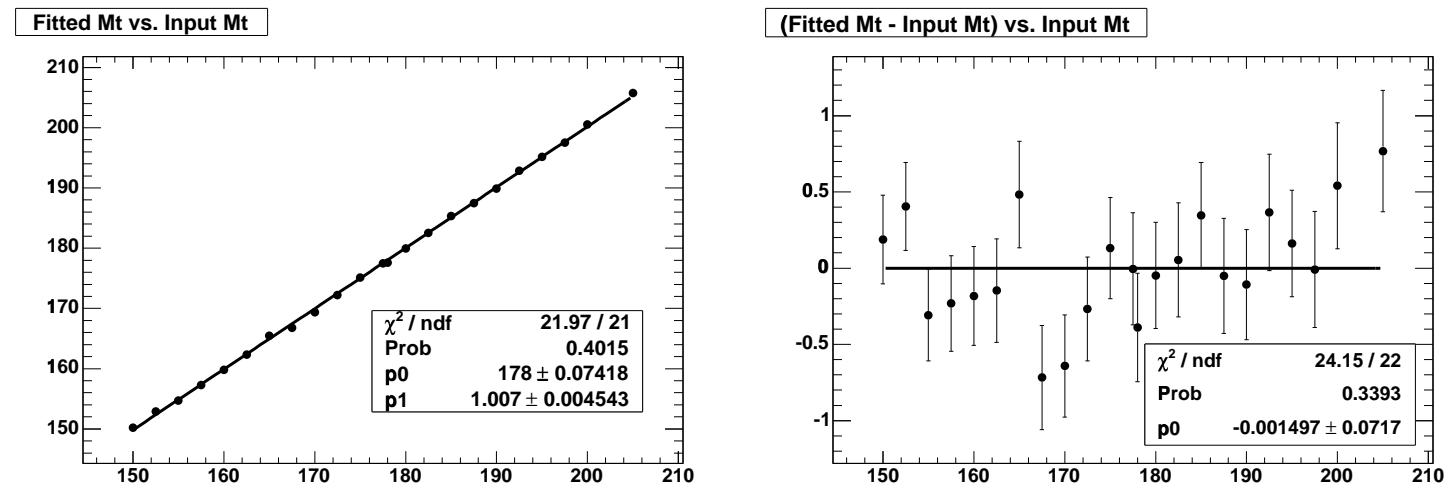

Figure 9.10: Fully simulated $t \bar{t}$ and background events analyzed with our full likelihood and mapping function. Pseudo-experiments of the 63-event style, 54.33 Poisson-mean signal events and backgrounds as per Table 4.3. On the left is the usual linearity test, fitted $m_{t}$ vs. input $m_{t}$. On the right is the same information plotted as (fitted $m_{t}$ - input $m_{t}$ ) vs. input $m_{t}$. The left-hand plot is fit to $00+\mathrm{p} 1 *$ (input $m_{t}-178$ ) and the right-hand plot to a constant.

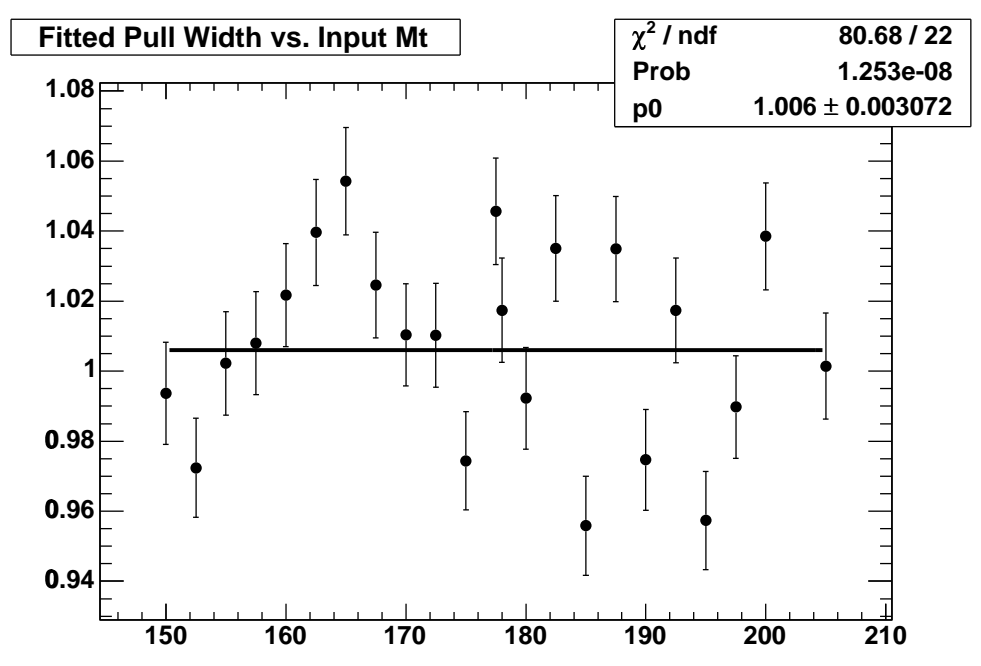

Figure 9.11: Fully simulated $t \bar{t}$ and background events analyzed with our full likelihood and mapping function. Pseudo-experiments of the 63-event style, 54.33 Poisson-mean signal events and backgrounds as per Table 4.3. Fitted widths of pulls from resampled pseudoexperiments. Error bars have been extracted directly from the fit to resampled pseudoexperiments, and not inflated as in other versions of this plot. 


\section{Fitted Pull Mean vs. Input Mt}

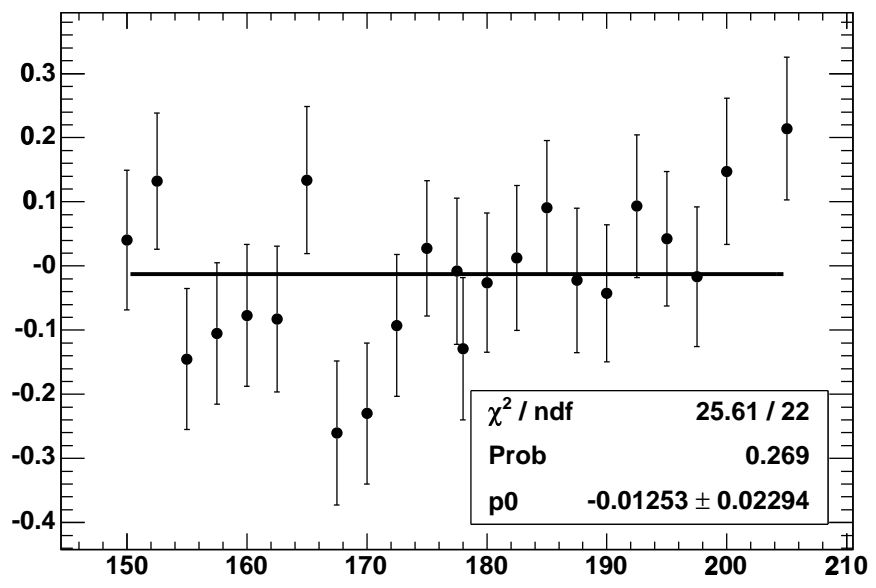

Figure 9.12: Fully simulated $t \bar{t}$ and background events analyzed with our full likelihood and mapping function. Pseudo-experiments of the 63-event style, 54.33 Poisson-mean signal events and backgrounds as per Table 4.3. Fitted means of pull distributions from resampled pseudo-experiments.

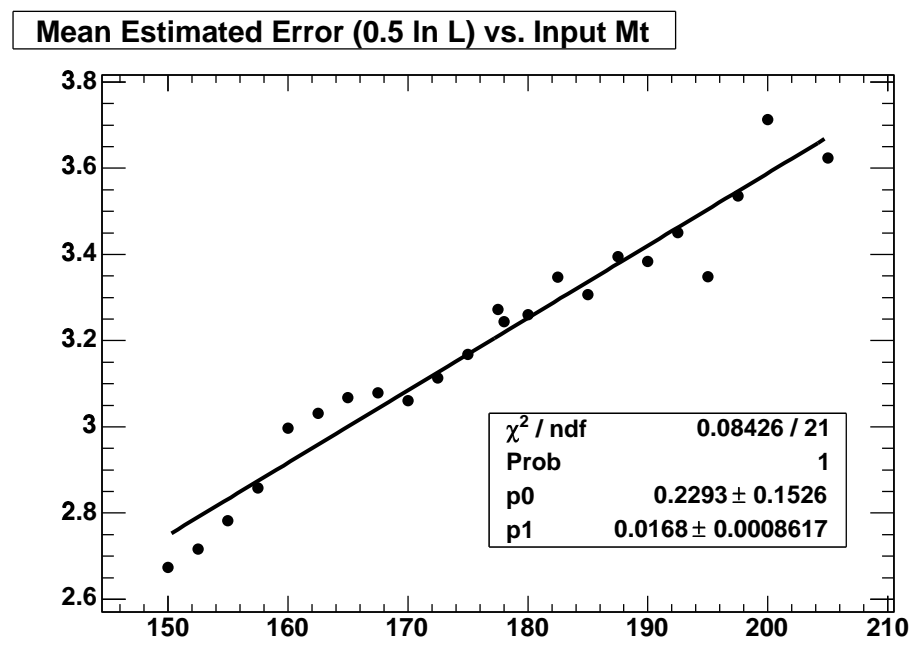

Figure 9.13: Fully simulated $t \bar{t}$ and background events analyzed with our full likelihood and mapping function. Pseudo-experiments of the 63-event style, 54.33 Poisson-mean signal events and backgrounds as per Table 4.3. Mean of estimated error distribution (i.e. mean expected error) as a function of $m_{t}$. This plot is after our mapping function has been applied, so the error is accurately estimated. 


\section{Chapter 10}

\section{Expected Error and Results from Data}

\section{$10.1 \quad$ Expected Error}

With the mapping functions established in Chapter 9 we are able to produce unbiased measurements of $m_{t}$ with accurately estimated errors for two types of pseudoexperiments: those applicable to the 63 events we observe in data, and those we would expect from a $6.1 \mathrm{pb} t \bar{t}$ cross section. To facilitate comparisons between methods, and to give a sense for the expected power of our method, we plot the estimated error from pseudo-

experiments at $m_{t}=178 \mathrm{GeV} / c^{2}$ for each of these two cases. We apply the mapping function appropriate to each case and in this chapter we always use pseudo-experiments with signal and all backgrounds, and analyze them with the full likelihood.

The $6.1 \mathrm{pb}$ case can be seen in Figure 10.1. There our mean expected error is 4.1 $\mathrm{GeV} / c^{2}$, and, crudely estimated, the median and most probable error are $4.0 \mathrm{GeV} / c^{2}$ and $3.8 \mathrm{GeV} / c^{2}$, respectively.

The 63-event case can be seen in Figure 10.2. There our mean expected error is $3.2 \mathrm{GeV} / c^{2}$, and, crudely estimated, the median and most probable error are $3.2 \mathrm{GeV} / c^{2}$ and $3.0 \mathrm{GeV} / c^{2}$, respectively.

We have already seen the mean expected error as a function of $m_{t}$ for 63 -event pseudo-experiments in Figure 9.13.

For comparison with the result from data, we also consider the 63-event cases for 


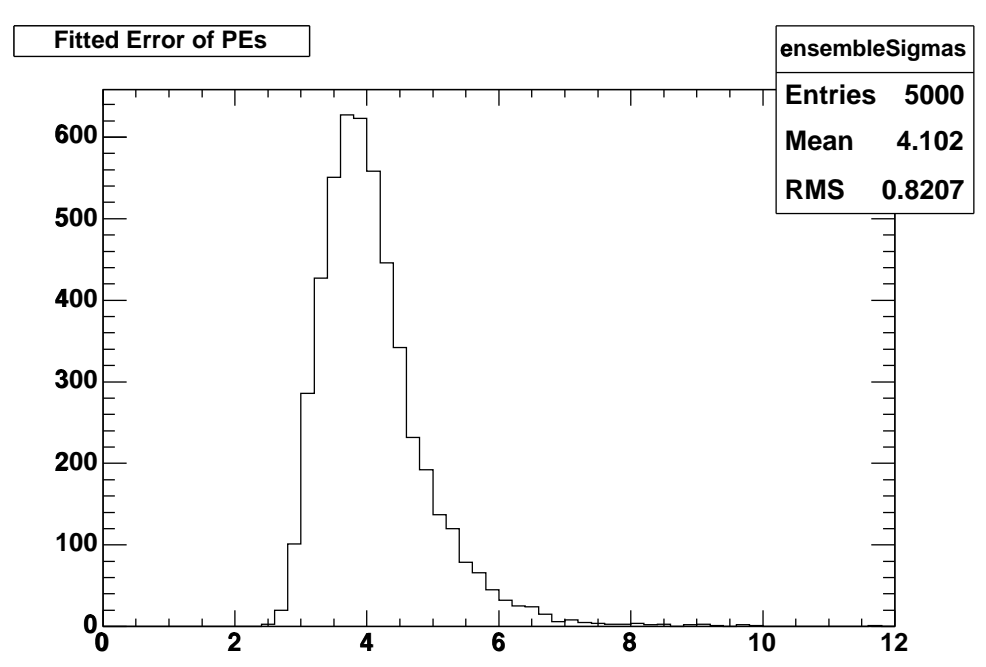

Figure 10.1: Expected error, from pseudo-experiments, of our measurement of $m_{t}$, assuming $m_{t}=178 \mathrm{GeV} / c^{2}$. Our mean expected error is $4.1 \mathrm{GeV} / c^{2}$, and, crudely estimated, the median and most probable error are $4.0 \mathrm{GeV} / c^{2}$ and $3.8 \mathrm{GeV} / c^{2}$, respectively. This is for the type of ensembles we expect with a $6.1 \mathrm{pb} t \bar{t}$ cross section.

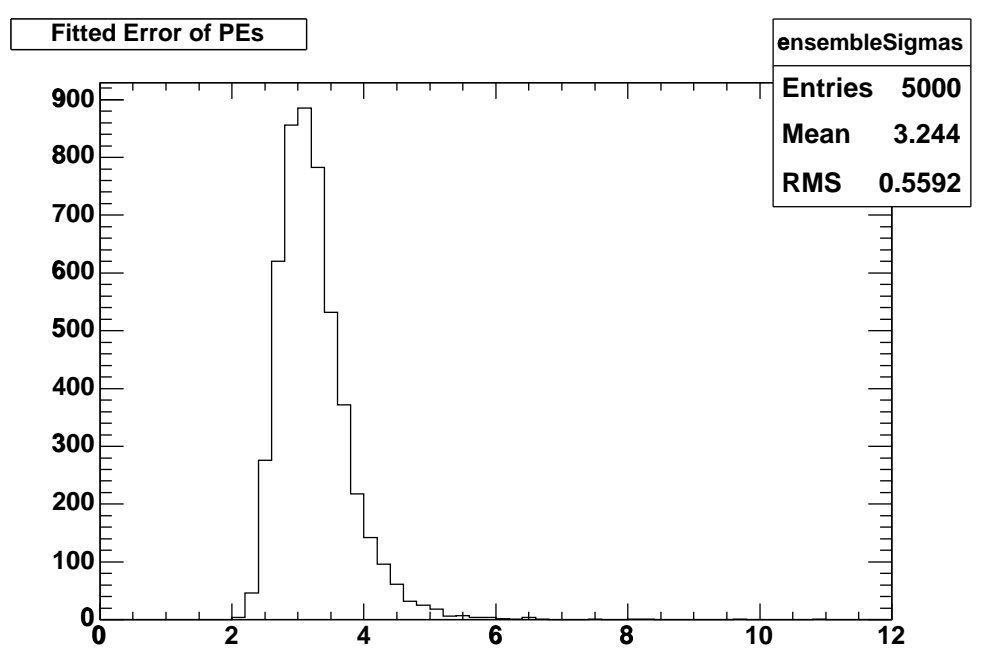

Figure 10.2: Expected error, from pseudo-experiments, of our measurement of $m_{t}$, assuming $m_{t}=178 \mathrm{GeV} / c^{2}$. Our mean expected error is $3.2 \mathrm{GeV} / c^{2}$, and, crudely estimated, the median and most probable error are $3.2 \mathrm{GeV} / c^{2}$ and $3.0 \mathrm{GeV} / c^{2}$, respectively. This is for the type of ensembles we expect based on the observed 63 events in data. 


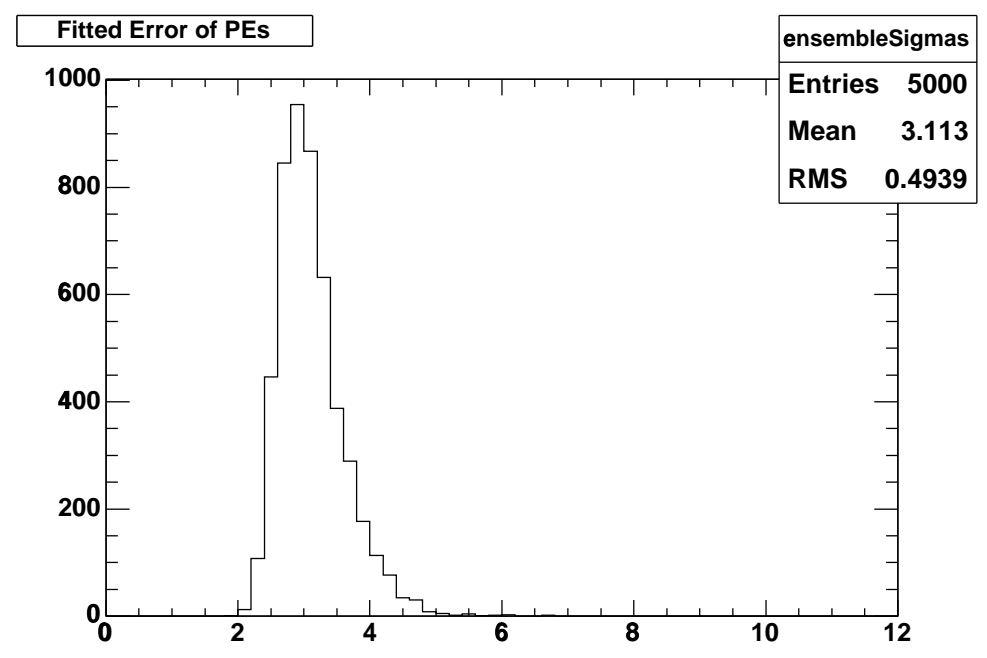

Figure 10.3: Expected error, from pseudo-experiments, of our measurement of $m_{t}$, assuming $m_{t}=172.5 \mathrm{GeV} / c^{2}$. Our mean expected error is $3.0 \mathrm{GeV} / c^{2}$, and the median expected error is $3.0 \mathrm{GeV} / c^{2}$. The expected error distribution has something of a broad peak, from 2.7 to $3.1 \mathrm{GeV} / c^{2}$, but we might estimate the most probable error as $2.9 \mathrm{GeV} / c^{2}$. This is for the type of ensembles we expect based on the observed 63 events in data, and the actual measurement in collider data can be compared to this distribution.

$m_{t}=172.5 \mathrm{GeV} / c^{2}$. This case can be seen in Figure 10.3. There our mean expected error is $3.1 \mathrm{GeV} / c^{2}$ and the median expected error is $3.0 \mathrm{GeV} / c^{2}$. The expected error distribution has something of a broad peak, from 2.7 to $3.1 \mathrm{GeV} / c^{2}$, but we might estimate the most probably error as $2.9 \mathrm{GeV} / c^{2}$.

\subsection{Results from Data}

The likelihood fit to the 63 events observed in $318 \mathrm{pb}^{-1}$ of data at CDF can be seen in Figures 10.4 and 10.5. These plots are after the mapping function has been applied. A list of the observed events in data may be found in Tables 4.1 and 4.2. The measured mass is $172.0 \pm 2.6$ (stat) $\mathrm{GeV} / c^{2}$.

Based on distributions of the expected error from pseudo-experiments, like that of Figure 10.3, we would expect to measure a smaller statistical error $11 \%$ of the time, when the top mass is $172.5 \mathrm{GeV} / c^{2}$. 


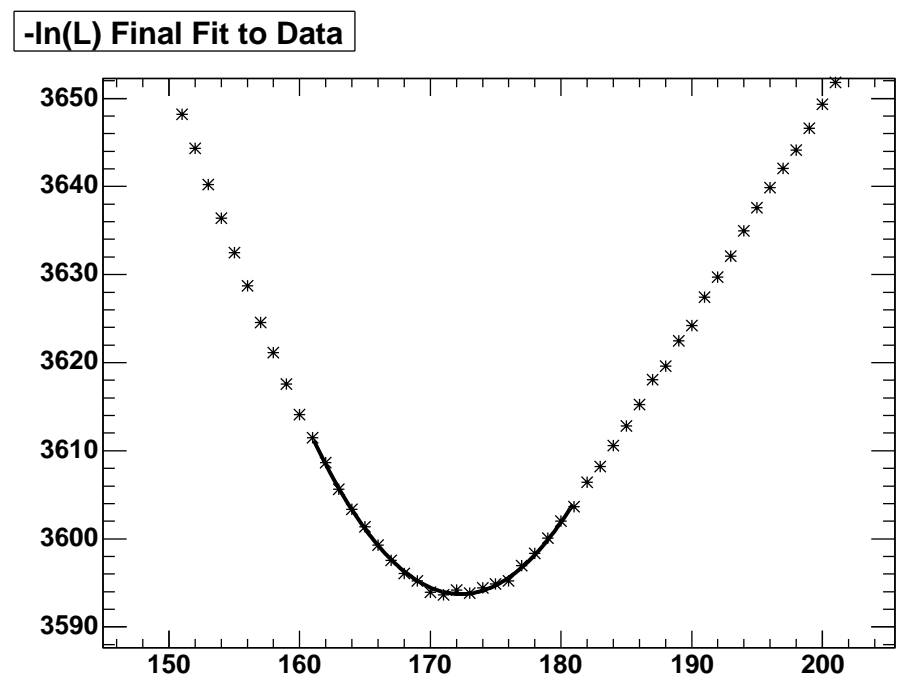

Figure 10.4: Likelihood fit to the 63 events observed in $318 \mathrm{pb}^{-1}$ of data at CDF. This is after the mapping function has been applied. The measured mass is $172.0 \pm 2.6$ (stat) $\mathrm{GeV} / c^{2}$.
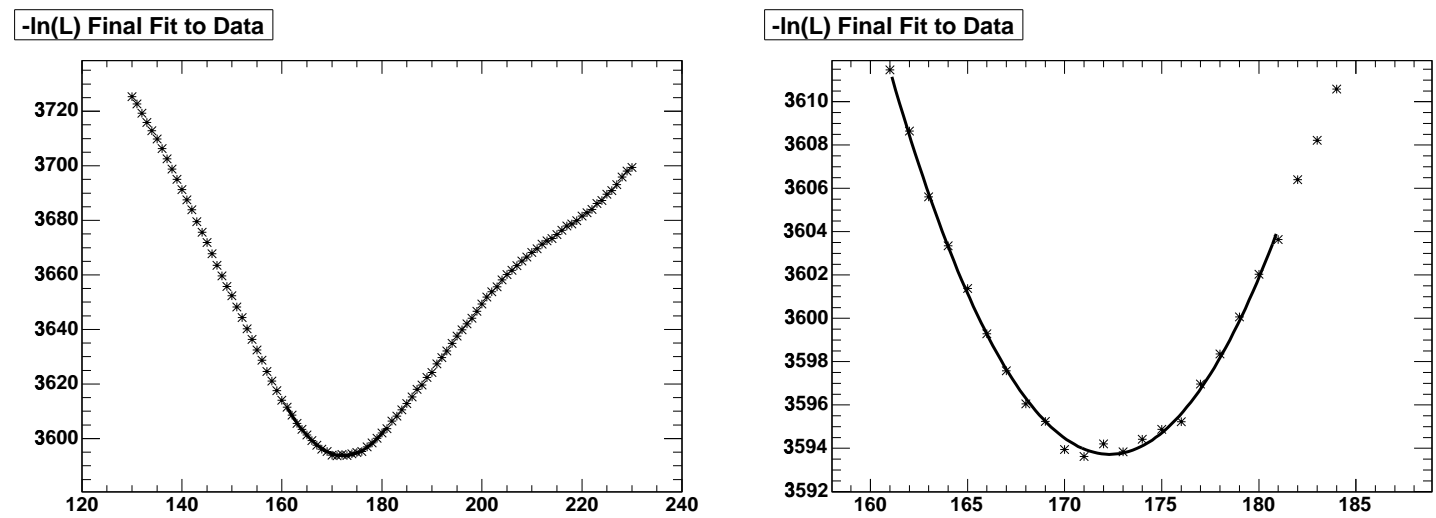

Figure 10.5: Alternative views of the likelihood fit to the 63 events observed in $318 \mathrm{pb}^{-1}$ of data at CDF. These are after the mapping function has been applied. The measured mass is $172.0 \pm 2.6$ (stat) $\mathrm{GeV} / c^{2}$. 


\section{Chapter 11}

\section{Systematic Uncertainty}

Our estimate of systematic errors is meant to include all those uncertainties which would survive in the limit of large statistics, that is all those errors which are not purely statistical. Since our method is calibrated against fully simulated Monte Carlo events we know that it works reliably, producing an unbiased $m_{t}$ measurement and an accurate estimation of the statistical error, for those events. So, the systematic uncertainty estimate codifies all possible differences between our fully simulated Monte Carlo events and actual data events.

Our procedure for estimating the magnitude of the effect of particular sources of systematic uncertainty reflects this. We leave our analysis machinery completely unchanged, and prepare samples of Monte Carlo events that reflect possible data-Monte Carlo uncertainties. In each case, we attempt to quantify an uncertainty that would reasonably cover a $\pm 1 \sigma$, that is a range within which we expect the truth to lie $68 \%$ of the time.

So, for example, possible differences in the jet energy scale between data and Monte Carlo are well studied. Monte Carlo samples are prepared with the jet energy scale systematically shifted, within an estimated $\pm 1 \sigma$ range, and are analyzed in the same way as the data events. So, we analyze 63-event pseudo-experiments with 50.58 Poissonmean signal events and 12.42 Poisson-mean background events as expected in the data. The analysis machinery is exactly that used for the data, as is the mapping procedure. The difference between the mean $m_{t}$ extracted from these systematically altered pseudoexperiments and the default Monte Carlo pseudo-experiments is taken as the systematic uncertainty associated with the jet energy scale. All systematics are estimated using Monte Carlo samples generated with $m_{t}=178 \mathrm{GeV} / c^{2}$. 
Many sources of possible systematic uncertainty affect a broad range of analyses at $\mathrm{CDF}$ and so they are handled uniformly across many analyses. For example, any analysis using jets is potentially affected by the uncertainty in the jet energy scale, and the effects are estimated in much the same way for all of these analyses. Because of this commonality across many CDF analyses we will not fully describe the estimation of these systematic uncertainties here. We will include a summary of these estimates below, emphasizing their effect on our analysis and providing appropriate references. These systematic effects for lepton + jets top mass analyses at CDF are summarized elsewhere in the literature [29].

\subsection{Jet Energy Scale}

Even with the latest innovations in jet energy scale measurements, including the in situ jet energy scale determination, the jet energy scale is the dominant source of systematic uncertainty for top mass measurements. Even though the jets forming the hadronic $W$ are relatively well constrained by the $W$ Breit-Wigner, the unconstrained $b$ jet allows an approximately linear dependence of $m_{t}$ on the jet energy scale. So, in many analyses including ours, a $1 \%$ uncertainty in the jet energy scale corresponds roughly to a $1 \mathrm{GeV} / c^{2}$ uncertainy in $m_{t}$.

The systematic uncertainty in the jet energy scale is determined using the same techniques as the corrections themselves (Section 4.4.2) and is described in detail elsewhere [59].

The systematic uncertainty in the $\eta$-dependent correction is estimated based upon the residual scatter in the di-jet and gamma-jet balancing after the correction has been applied, and based upon the differences observed between these two balancing techniques. In general the systematic uncertainties on the jet energy scale are based upon the limited statistics of those corrections extracted from data and on assumptions invoke when extracting the corrections. Systematic uncertainties are thus estimated for the multiple interactions correction and the "absolute correction" which corrects for calorimeter non-linearities. Even though we do not explicitly apply the generic CDF corrections for the underlying event (the residual $p$ and $\bar{p}$ fragments apart from the hard-scattering events) or for energy that falls outside the jet cone of radius 0.4 (the out-of-cone and splash-out corrections) we still must consider their systematic uncertainties. These systematic uncertainties reflect potential differences between data and Monte Carlo and so represent possible differences between our 


\begin{tabular}{|c|c|c|c|}
\hline Description & $-1 \sigma$ & $+1 \sigma$ & Systematic $\left(\mathrm{GeV} / c^{2}\right)$ \\
\hline$\eta$-Dependence & 177.35 & 178.33 & 0.49 \\
\hline Multiple Interactions & 177.78 & 177.85 & 0.04 \\
\hline Absolute Scale & 175.47 & 179.66 & 2.09 \\
\hline Underlying Event & 177.65 & 178.07 & 0.21 \\
\hline Out-of-Cone & 175.64 & 179.67 & 2.02 \\
\hline Splash-Out & 177.52 & 177.95 & 0.21 \\
\hline \multicolumn{3}{|c|}{ Sum of the Above } & 2.96 \\
\hline From TotalSys Method & 174.46 & 180.62 & 3.08 \\
\hline
\end{tabular}

Table 11.1: Estimated systematic error due to uncertainty in the jet energy scale. The total systematic is given as well as the breakdown into individual components. If we treated the samples as uncorrelated, the statistical error for each would be about $0.35 \mathrm{GeV} / c^{2}$. But, in reality they are highly correlated, all coming from the same underlying signal and background samples, and so we neglect the statistical error. We take a $3.08 \mathrm{GeV} / c^{2}$ systematic error.

transfer function, extracted from Monte Carlo, and the real data events.

For each of our signal and background samples we shift the jet energy scale by $\pm 1 \sigma$, where $\sigma$ is the estimated $68 \%$ confidence level of the source of systematic uncertainty, before our event selection is applied. Then, we analyze the events as usual and extract $m_{t}$. By default we apply all of the various individual sources of systematic uncertainty at the same time, and in the same direction. This reflects an overall uncertainty in the jet energy scale of approximately $3 \%$. We take half of the difference between the two sets $(+1 \sigma$ and $-1 \sigma$ as a systematic error. As a cross-check, we also check the uncertainty due to each individual level of the jet energy corrections. Results can be found in Table 11.1.

The systematic due to uncertainty on the multiple interactions correction is underestimated by our methods, since the default $t \bar{t}$ Monte Carlo underestimates the number of interactions per event. This effect was investigated in the context of the Dynamical Likelihood Method analysis by consider $t \bar{t}$ samples where two extra minimum bias events have been explicitly added to each $t \bar{t}$ event. In this way, they estimated the systematic to be 0.1 $\mathrm{GeV} / c^{2}[76]$, which we can safely neglect. Overall, we take a $3.08 \mathrm{GeV} / c^{2}$ systematic error due to the uncertainty in jet energy scale. 


\begin{tabular}{|c|c|}
\hline Sample & Measured $m_{t}\left(\mathrm{GeV} / c^{2}\right)$ \\
\hline PYTHIA & $177.44 \pm 0.36$ \\
\hline HERWIG & $177.61 \pm 0.37$ \\
\hline \hline Systematic & $0.2 \pm 0.5 \mathrm{GeV} / c^{2}$ \\
\hline
\end{tabular}

Table 11.2: Estimated systematic error due to differences in event generators and parton showering codes. Conservatively, we take a $0.5 \mathrm{GeV} / c^{2}$ systematic uncertainty.

\section{$11.2 \quad b$ Jet Energy Scale}

The jet energy scale uncertainties that we have just discussed are primarily focused on jets generically, meaning mostly jets originating from light quarks and gluons. There are additional potential sources of uncertainty that affect heavy flavor jets. Detailed studies of the $b$ jet energy scale uncertainties associated with uncertainties in heavy quark fragmentation, uncertainties semi-leptonic decays, and in color flow are summarized in elsewhere [77]. There is found a $0.2 \pm 0.4 \mathrm{GeV} / c^{2}$ uncertainty due to fragmentation, a $0.4 \%$ uncertainty on the b-jet energy scale due to uncertainty in the semi-leptonic branching fraction, and a $0.3 \%$ uncertainty on the $b$-jet energy scale due to uncertainties in the modelling of the color flow in $t \bar{t}$ events. As for that template-based analysis, in our analysis, to a good approximation, a $1 \%$ uncertainty in the jet energy scale corresponds to a $1 \mathrm{GeV} / c^{2}$ uncertainty in $m_{t}$. So, we add these three effects in quadrature and take a $0.6 \mathrm{GeV} / c^{2}$ systematic due to uncertainties specific to the $b$-jet energy scale.

\subsection{Generator}

At a given order of theory, especially leading order, the hard scattering process is relatively unambiguous. But, the fragmentation and hadronization process is much less so. At CDF this part of the simulation is handled by two parton shower codes, PYTHIA and HERWIG. HERWIG and PYTHIA employ different fragmentation and hadronization models and are also tuned somewhat differently to experimental data. We consider the difference in measured $m_{t}$ between HERWIG and PYTHIA $t \bar{t}$ samples as a systematic error. Results can be found in Table 11.2. The two are consistent with each other, but the comparison is dominated by the statistical error. Conservatively, we take a $0.5 \mathrm{GeV} / c^{2}$ systematic error due to the uncertainty in generator modelling of the underlying physics. 


\begin{tabular}{|c|c|}
\hline Source & Measured $m_{t}\left(\mathrm{GeV} / c^{2}\right)$ \\
\hline Less ISR & $177.34 \pm 0.34$ \\
\hline More ISR & $177.80 \pm 0.35$ \\
\hline Systematic & $0.4 \pm 0.5 \mathrm{GeV} / c^{2}$ \\
\hline \hline Less FSR & $177.73 \pm 0.34$ \\
\hline More FSR & $177.90 \pm 0.33$ \\
\hline \hline Systematic & $0.5 \pm 0.5 \mathrm{GeV} / c^{2}$ \\
\hline \hline PYTHIA Default & $177.44 \pm 0.36$ \\
\hline
\end{tabular}

Table 11.3: Estimated uncertainty due to initial- and final-state radiation. Since the $\pm 1 \sigma$ do not bracket the default sample, we take the largest difference between the $\pm 1 \sigma$ samples and the default as the systematic. So, we take $0.5 \mathrm{GeV} / c^{2}$ each for initial- and final-state radiation.

\subsection{Radiation}

We estimate the uncertainty due to the uncertainty in quantity and characteristics of initial and final state radiation by considering samples generated for this purpose [78]. The systematic uncertainty in the amount of initial-state radiation is driven by special studies of Drell-Yan events at CDF as a function of $p_{T}$. A hard gluon radiated by one of the initial-state quarks or gluons imparts additional transverse momentum to the entire system involved in the hard-scattering event. Samples are generated to bracket the experimental uncertainty, with more and less initial-state radiation than the default Monte Carlo. The samples with more and less final-state radiation were generated in consultation with the PYTHIA authors to generously bracket uncertainties in final-state radiation based on worldwide knowledge of such effects. All of the radiation systematic samples are from PYTHIA. Results can be found in Table 11.3. Since the $\pm 1 \sigma$ do not bracket the default sample, we take the largest difference between the $\pm 1 \sigma$ samples and the default as the systematic. These comparisons, unfortunately, have a statistical error comparable to the observed shift. We estimate our systematic uncertainties as $0.5 \mathrm{GeV} / c^{2}$ due to initial-state radiation and $0.5 \mathrm{GeV} / c^{2}$ due to final-state radiation. 


\begin{tabular}{|c|c|}
\hline Fraction of $g g$ events & Measured $m_{t}\left(\mathrm{GeV} / c^{2}\right)$ \\
\hline $0 \%$ & $177.41 \pm 0.37$ \\
\hline $5 \%$ & $177.61 \pm 0.37$ \\
\hline $10 \%$ & $177.83 \pm 0.53$ \\
\hline $15 \%$ & $177.97 \pm 0.65$ \\
\hline $20 \%$ & $178.23 \pm 0.67$ \\
\hline $25 \%$ & $178.44 \pm 0.74$ \\
\hline $100 \%$ & $181.61 \pm 1.51$ \\
\hline \hline Systematic & $0.4 \mathrm{GeV} / c^{2}$ \\
\hline
\end{tabular}

Table 11.4: Measured $m_{t}$ for samples with various $g g$ fractions. The quoted statistical errors assume the samples are independent, when in reality they are all drawn from the same sample of events. But, the precision is limited by the small number of $g g$ events available. Comparing the values at $5 \%$ and $15 \%$ we estimate a systematic uncertainty of $0.4 \mathrm{GeV} / c^{2}$.

\section{5 $t \bar{t}$ Production via Gluon Fusion $(g g)$ vs. Quark Anti- Quark Annihilation ( $q q)$}

In our default leading order $t \bar{t}$ Monte Carlo samples we expect, and observe, about $5 \%$ of events to be produced via gluon fusion. In the data, as in next-to-leading order calculations, we expect that the fraction is closer to $15 \%$. Our signal probability takes into account only $q q$ production and is then claibrated on $t \bar{t}$ Monte Carlo with the leading order $5 \% g g$ fraction. Having this fraction wrong in our calibration samples can potentially bias the $m_{t}$ measurement. So, we prepare samples with various fractions of $g g$ events.

Our results can be found in Table 11.4. Comparing the values at 5\% and 15\% we estimate a systematic uncertainty of $0.4 \mathrm{GeV} / c^{2}$.

This systematic, for the fraction of $g g$ events in the data, could be considered an example of a next-to-leading order systematic. Our signal probability uses a leading order signal probability, but this by itself does not introduce a systematic uncertainty since we calibrate this probability. The systematic is, instead, due to potential differences between our simulated samples and data. Here we have considered differences between $g g$ and $q q$ events. We expect that the difference between the leading order and next-to-leading order $t \bar{t}$ cross section is primarily a matter of total cross section and $g g$ vs. $q q$ fraction. Slight kinematic differences are also possible, and future CDF $m_{t}$ measurements will examine next-to-leading order $t \bar{t}$ samples generated with MC@NLO [79, 80] to address this question. 
Preliminary studies have shown no large difference with our default leading order samples, generated with HERWIG.

In the future we could consider incorporating a next-to-leading order signal probability to improve the precision of the measurement. But, this is computationally daunting.

\subsection{Uncertainties in Parton Density Functions}

In recent years, the groups that produce Parton Density Function (PDF) fits have made the job of quantitatively estimating the effects of uncertainties in PDF fits much easier. The CTEQ collaboration has identified 20 eigenvectors, corresponding to each component of their global fit to world data sets, and produced PDF's that correspond to a $+1 \sigma$ and a $-1 \sigma$ variation in the eigenvalue for each [81]. So, as well as their default leading order and next-to-leading order PDF's they have forty PDF's designed to facilitate the estimation of systematic error. These forty PDF's for systematic errors are fluctuations related to their sixth generation next-to-leading order PDF, CTEQ6M.

Generating fully simulated $t \bar{t}$ samples using each of these forty PDF's would be computationally daunting, so we instead use a reweighting technique. The reweighting technique begins with the PYTHIA version of our default Monte Carlo sample, based on the CTEQ5L PDF. We modify our pseudo-experiment to procedure to admit an event to a pseudo-experiment statistically, based on its weight for the PDF being considered. In this fashion, we can construct ensembles of pseudo-experiments which are very highly correlated, with the only difference being the frequency with which individual events appear in the pseudo-experiments. To take advantage of the the correlations, we produce a very large number of pseudo-experiments $(50,000)$ for each PDF.

Our results may be found in Figure 11.1. Set 0 is the default CTEQ5L, sets 1 and 2 are MRST72 and MRST75 [82], sets 3 and 4 are CTEQ6L and CTEQ6L1, set 5 is CTEQ6M, and sets $6-45$ are the $\pm 1 \sigma$ variations on the 20 different CTEQ6M eigenvectors. Set 6 is $+1 \sigma$ for eigenvector 1 , set 7 is $-1 \sigma$ for eigenvector 1 , and so on. We take half the difference between the two versions of each eigenvector, and sum in quadrature, for a total systematic of $0.08 \mathrm{GeV} / c^{2}$ due to the CTEQ6M variations.

CTEQ5L and MRST72 are two leading order PDF's constructed from similar experimental input, but with somewhat different methodology, and by separate sets of authors. They should produce similar results, but since we see a difference in Figure 11.1 


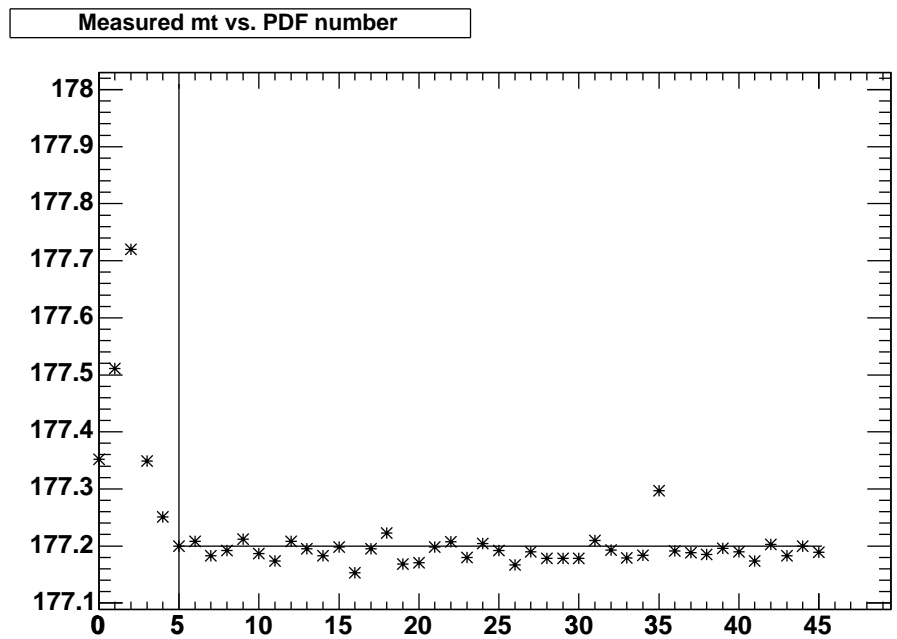

Figure 11.1: Measured $m_{t}$ for 46 different parton distribution functions (PDF's), compared using a reweighting technique. Set 0 is the default CTEQ5L, sets 1 and 2 are MRST72 and MRST75, sets 3 and 4 are CTEQ6L and CTEQ6L1, set 5 is CTEQ6M, and sets 6-45 are the $\pm 1 \sigma$ variations on the 20 different CTEQ6M eigenvectors. Set 6 is $+1 \sigma$ for eigenvector 1 , set 7 is $-1 \sigma$ for eigenvector 1 , and so on. We take half the difference between the two versions of each eigenvector, and sum in quadrature, for a total systematic of $0.08 \mathrm{GeV} / c^{2}$ due to the CTEQ6M variations. Comparing CTEQ5L to MRST72 we take a systematic of 0.16 $\mathrm{GeV} / c^{2}$, and comparing MRST72 and MRST75 take a systematic of $0.21 \mathrm{GeV} / c^{2}$. Adding these in quadrature, we take a total systematic uncertainty due to parton distribution function uncertainties of $0.3 \mathrm{GeV} / c^{2}$. 


\begin{tabular}{|c|c|}
\hline Source & Measured $m_{t}\left(\mathrm{GeV} / c^{2}\right)$ \\
\hline MRST72 $\left(\Lambda_{Q C D}=228 M e V\right)$ & $177.12 \pm 0.36$ \\
\hline MRST75 $\left(\Lambda_{Q C D}=300 M e V\right)$ & $177.32 \pm 0.33$ \\
\hline PYTHIA Default $($ CTEQ5L $)$ & $177.44 \pm 0.36$ \\
\hline
\end{tabular}

Table 11.5: Results from independently generated sets of fully simulated PYTHIA Monte Carlo events, generated with different parton distribution functions. The comparison is dominated by the statistical uncertainty. The fully simulated samples are consistent with each other, and also consistent with the results from the reweighting studies.

we take the difference, $0.16 \mathrm{GeV} / c^{2}$, as a systematic.

Finally, the 20 sets of CTEQ6M eigenvectors neglect what is often considered the most important uncertainty in extracting PDF's, that due to the uncertainty in $\Lambda_{Q C D}$. So, we also consider the difference between MRST72 $\left(\Lambda_{Q C D}=228 \mathrm{MeV}\right)$ and MRST75 $\left(\Lambda_{Q C D}=300 \mathrm{MeV}\right)$ as a systematic, $0.21 \mathrm{GeV} / c^{2}$.

Adding the three sources in quadrature, our total estimated uncertainty due to parton distribution functions is $0.3 \mathrm{GeV} / c^{2}$.

As a cross-check, we also consider the results from independently generated sets of fully simulated PYTHIA MC generated with CTEQ5L, MRST72, and MRST75. The results can be found in Table 11.5. This comparison is dominated by the statistical uncertainty, but is trivially consistent with the conclusions from the reweighting studies.

\subsection{Background Composition}

For the $b$ tagged lepton + jets sample the background normalization and composition are determined with good, but not infinite, precision. To estimate the uncertainty on $m_{t}$ we vary the amount of each background within its uncertainty. The central values and errors of each background normalization can be found in Table 4.3. In each case, the mean size of the pseudo-experiment is 63 events - the number of events we observe in data. For example, our default pseudo-experiments have 54.33 Poisson-mean $t \bar{t}$ events and Poisson-mean $1.59 b \bar{b}$ events. For the $W b \bar{b}+1 \sigma$ case we use $53.58 t \bar{t}$ events and $2.34 b \bar{b}$ events.

Results can be found in Table 11.6 where we also allow the total background normalization to float, with $\pm 1 \sigma$ values of 10.40 and 6.94 events (background fraction varying from $11.0 \%$ to $16.5 \%$ ). If we treat each case as statistically independent they 


\begin{tabular}{|c|c|c|c|}
\hline Background & $-1 \sigma$ & $+1 \sigma$ & Systematic $\left(\mathrm{GeV} / c^{2}\right)$ \\
\hline$W 4 p$ & 177.65 & 177.62 & 0.02 \\
\hline$W b \bar{b}$ & 177.64 & 177.59 & 0.04 \\
\hline QCD & 177.60 & 177.66 & 0.03 \\
\hline$W c \bar{c}$ & 177.65 & 177.63 & 0.02 \\
\hline$W c$ & 177.64 & 177.60 & 0.03 \\
\hline Single Top & 177.64 & 177.62 & 0.01 \\
\hline EW & 177.64 & 177.65 & 0.02 \\
\hline \hline Global Shift & 177.69 & 177.62 & 0.07 \\
\hline \hline Default & \multicolumn{3}{|c|}{177.63} \\
\hline
\end{tabular}

Table 11.6: Estimated systematic error due to uncertainties in the composition of the background contribution to the $t \bar{t}$ sample. For each line we have shifted the amount of a particular background by $\pm 1 \sigma$. We have also shifted the overall background normalization by $\pm 1 \sigma$ while holding the relative composition constant, for the "Global Shift" line. If we treat the cases as statistically independent they would typically have a statistical error of $0.3-0.4 \mathrm{GeV} / c^{2}$. However, they are highly correlated, taking very similar numbers of events from all of the sample samples. So, instead we neglect the statistical uncertainty on the individual entries. Conservatively, we take the maximum deviation between the $\pm 1 \sigma$ samples and the default as a systematic for each background. Adding them in quadrature we take an overall systematic due to background composition of $0.1 \mathrm{GeV} / c^{2}$.

would typically have a statistical error of $0.3-0.4 \mathrm{GeV} / c^{2}$. However, they are in fact highly correlated, taking very similar numbers of events from all of the same samples. So, instead we neglect the statistical uncertainty on the individual entries. As in the case of the PDF uncertainties (Chapter 11.6) we generate a very large number of pseudo-experiments to take advantage of the large correlations, and to help identify any small differences due to the composition of the background.

Conservatively, we take the maximum deviation between the $\pm 1 \sigma$ samples and the default as a systematic for each background. Adding them in quadrature we take an overall systematic due to background composition of $0.1 \mathrm{GeV} / c^{2}$.

\subsection{Background Modelling}

To give some sense of our sensitivity to how well our Monte Carlo background samples represent what is actually present in data we consider an extreme case. We generate pseudo-experiments where all of our 8.67 expected background events are selected from the same background sample, and compare these pseudo-experiments to the default case. 


\begin{tabular}{|c|c|}
\hline Background & Mass $\left(\mathrm{GeV} / c^{2}\right)$ \\
\hline$W 4 p$ & $177.58 \pm 0.37$ \\
\hline$W b \bar{b}$ & $177.59 \pm 0.37$ \\
\hline $\mathrm{QCD}$ & $177.94 \pm 1.1$ \\
\hline$W c \bar{c}$ & $177.50 \pm 0.57$ \\
\hline$W c$ & $177.46 \pm 0.62$ \\
\hline Single Top & $177.58+-0.36$ \\
\hline EW & $177.15+-1.0$ \\
\hline \hline Default & $177.61 \pm 0.37$ \\
\hline
\end{tabular}

Table 11.7: Pseudo-experiments where the total amount of expected background is drawn from individual background samples, instead of the usual mixture. The listed errors are statistical only. The signal events used in the various sets of pseudo-experiments are 100\% correlated, while the background events are, of course, not. We take a systematic uncertainty of $0.5 \mathrm{GeV} / c^{2}$, the largest difference between any of the individual tests and the default.

The results can be found in Table 11.7. The listed errors are statistical only, and assume the different sets of pseudo-experiments are statistically independent. In actuality, the signal events are highly correlated between sets of pseudo-experiments while the background events, of course, are not. The statistics for most of the samples could be improved by relaxing the $b$ tag requirement, but not, unfortunately, for the QCD sample, where we have already relaxed this requirement. The largest deviations are for the case where we consider all the background to have come from QCD or electroweak, each with a deviation of about $0.5 \mathrm{GeV} / c^{2}$. This test, of taking all of our background events from individual background samples, is certainly extreme. It assumes, for example, that our background single top sample no better a description of the single top events in data than it is of the electroweak or QCD events. Conservatively, we take a $0.5 \mathrm{GeV} / c^{2}$ systematic error due to uncertainties in background modelling.

\subsection{Monte Carlo Statistics}

The number of pseudo-experiments used to determine the error on the mean of $m_{t}$ distributions is based upon the available statistics in our $t \bar{t}$ samples, and we typically have about 78 exclusive $t \bar{t}$ pseudo-experiments available. However, with this estimation we do ignore the even more limited statistics available for one of our backgrounds, the non$W$ QCD sample which is taken from the set of non-isolated electron candidate events in 


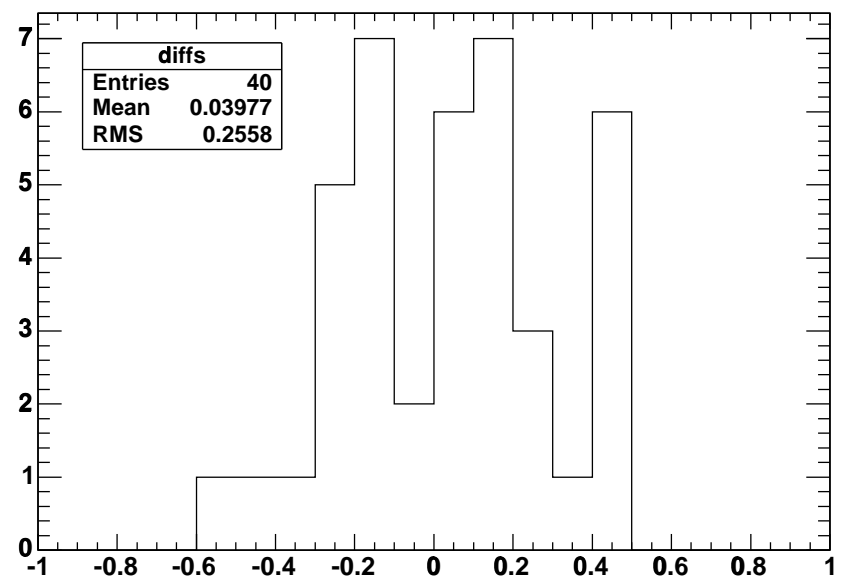

Figure 11.2: To estimate the effect of the limited size of our non- $W$ QCD sample we divide our sample into two exclusive sub-samples, and measure $m_{t}$ with an ensemble of pseudo-experiments from each. The difference between the mean $m_{t}$ of the two ensembles is plotted above. We repeat this procedure forty times. We take the RMS of this distribution, divided by $\sqrt{2}$ since our actual sample is twice the size of these sub-samples, as a systematic uncertainty of $0.2 \mathrm{GeV} / c^{2}$.

data. Here, we only have about 25 exclusive pseudo-experiments available. So, all of our estimations involve resampling of the QCD sample. And, in this sense the error quoted on the mean of $m_{t}$ distributions from pseudo-experiments is underestimated.

To estimate the consequences of this, we divide our QCD events in two and measure $m_{t}$ from an ensemble of pseudo-experiments using each exclusive set. We consider the difference between these two measurements. We repeat this procedure 40 times, with 40 different binary divisions. Our results can be found in Figure 11.2.

We take the RMS of this distribution as an estimate of the statistical uncertainty associated with using a QCD sample half the size of the one we use for our usual pseudoexperiments. So, we divide the RMS by $\sqrt{2}$ and estimate the uncertainty as $0.18 \mathrm{GeV} / c^{2}$. We take a $0.2 \mathrm{GeV} / c^{2}$ systematic due to the limited statistics of our non- $W$ QCD sample. Because of our resampling of QCD background events when we generate pseudo-experiments the error on the mean $m_{t}$ value extracted from any set of pseudo-experiments is underestimated by $0.2 \mathrm{GeV} / c^{2}$.

In the future, we should consider a new set of cuts when extracting our QCD sample from data, in order to have a more abundant sample. 


\subsection{Uncertainty on Mapping Function}

The uncertainty on the magnitude of our default, flat mapping function ${ }^{1}$ is only $70 \mathrm{MeV}$. But, since the $\chi^{2}$ probability for a linear mapping function is slightly better, we consider the difference between our default and the linear case as a systematic uncertainty. At the extreme masses of 150 and $205 \mathrm{GeV} / c^{2}$ the difference is -160 and $+210 \mathrm{MeV}$. So, we take a conservative systematic uncertainty of $0.2 \mathrm{GeV} / c^{2}$.

\section{$11.11 b$ Tagging Scale Factor Uncertainty}

The $b$ tagging scale factor, the ratio between the tagging rate in data and simulation, is assumed to be flat for all $b$ jet $E_{T}$. But, if the tagging rate in data is different in data in a way that depends on the $B$ jet energy it would bias our measurement. So, we consider the bounding cases of

$$
\epsilon^{+}=0.842+0.00118 \times E_{T}
$$

and

$$
\epsilon^{-}=0.977-0.00118 \times E_{T}
$$

where $\epsilon$ is the $b$ tagging scale rate, $E_{T}$ is the $b$ jet energy (before the transfer function or the absolute correction back to particle-jet level are applied). In no case is the scale factor considered to be larger than 1.00.

We estimate the systematic uncertainty due to such a variation with a similar reweighting technique to that we used for the PDF uncertainty (see Chapter 11.6. We assign each event a weight according to the $E_{T}$ of the tagged $b$ jets and generate a large ensemble of pseudo-experiments, from the same underlying samples, with the weighting applied. Our results can be found in Table 11.8. Taking half the difference between the two cases, we estimate a systematic uncertainty of $0.1 \mathrm{GeV} / c^{2}$.

\subsection{Summary of Systematic Errors}

See Table 11.9 for a summary of our systematic errors. The total systematic error is the sum in quadrature of the individual error estimates. We estimate the total systematic uncertainty on $m_{t}$ to be $3.3 \mathrm{GeV} / c^{2}$.

\footnotetext{
${ }^{1}$ Our mapping procedure is described in Chapter 9.2.
} 


\begin{tabular}{|c|c|}
\hline Sample & Measured $m_{t}\left(\mathrm{GeV} / c^{2}\right)$ \\
\hline$b$ tag scale factor increases with $E_{T}$ & 177.80 \\
\hline$b$ tag scale factor decreases with $E_{T}$ & 177.53 \\
\hline
\end{tabular}

Table 11.8: We estimate $m_{t}$ for cases in which we assume that the $b$ tag scale factor, the ratio between the $b$ tag efficiency in data and Monte Carlo simulation, increases or decreases with $E_{T}$. We take half the difference as a systematic, $0.1 \mathrm{GeV} / c^{2}$.

\begin{tabular}{|c|c|}
\hline Source of Systematic & Magnitude $\left(\mathrm{GeV} / c^{2}\right)$ \\
\hline Jet Energy Scale & 3.08 \\
\hline$b$ Jet Energy Scale & 0.6 \\
\hline Generator & $0.2 \pm 0.5$ \\
\hline ISR & $0.4 \pm 0.5$ \\
\hline FSR & $0.5 \pm 0.5$ \\
\hline Gluon Fraction & 0.4 \\
\hline PDF's & 0.3 \\
\hline Background Composition & 0.1 \\
\hline Background Modelling & 0.5 \\
\hline MC Statistics & 0.2 \\
\hline Mapping Function & 0.2 \\
\hline$b$ tag $E_{T}$ dependence & 0.1 \\
\hline \hline Total & $3.3 \mathrm{GeV} / c^{2}$ \\
\hline
\end{tabular}

Table 11.9: Summary of Systematic Errors 


\section{Chapter 12}

\section{Additional Checks}

\subsection{Understanding the Widths of Pull Distributions}

Since our likelihood uses leading-order matrix elements we know that it does not describe the complete picture of $t \bar{t}$ production and decay. For example, there is no allowance for initial- or final-state radiation in our matrix element. We make some additional assumptions in the kinematic reconstruction of $t \bar{t}$ production and decay based on selected quantities measured with our detector. We assume that the $p_{T}$ of the $t \bar{t}$ system is 0 and this, along with our assumption of no radiation, means that the $p_{T}$ of the neutrino is just the opposite of the sum of the $p_{T}$ 's of the four partons and the lepton. That is, under our assumptions, the $p_{T}$ of the neutrino exactly balances the $p_{T}$ of the other $t \bar{t}$ decay products. We use transfer functions to model the relation between parton and jet energies, but we assume that the jet angles correspond exactly to the parton angles. We assume that lepton angles and energies are well measured. Additionally, our signal probability ignores all $t \bar{t}$ decays except that to $e$ or $\mu+$ jets. So, in our likelihood we choose not to model dilepton and all-hadronic $t \bar{t}$ events, and events with $\tau$ 's ${ }^{1}$ Similarly we model only $q \bar{q}$ production and ignore the $g g$ initial-state.

We expect all of these assumptions to be violated, to varying degrees, in fully simulated events and in the real events observed by our detector. To the extent that these assumptions are violated our likelihood becomes only approximate: the probability encoded in the likelihood is not the real probability that nature (or our chain of matrix-

\footnotetext{
${ }^{1}$ Of course, dilepton, all-hadronic, and $\tau+$ jets events are heavily suppressed by our selection criteria, but they are present, to some extent, in our final data set.
} 
element generation, parton-showering, and full detector simulation) uses to generate the events. This means that we can not assume that our approximate likelihood will behave as we expect mathematically from the maximum likelihood method. In particular, the width of the pull distributions may be larger than unity and some bias of the mean may occur.

In principle it should be possible to model most of these additional effects in our likelihood. For example, we could attempt to address the issue of radiation by using a nextto-leading-order matrix element. Or, we could attempt to address the issue of measured jet angles not corresponding to parton angles (because of detector angular resolution as well as radiation and hadronization of the partons) by including transfer functions for the measured jet angles. We have addressed the least well measured component, jet energies, with transfer functions already. Some of these other omissions and approximations will be revisited, as potential upgrades, in future versions of this analysis.

But, while our likelihood remains approximate, that is while there are physical effects and processes that we choose not to model, we need to use fully simulated Monte Carlo events to study the effect of these approximations. Of course, these are exactly the studies we have described in Chapter 9 and they resulted in the mapping procedure that we use on data. This mapping procedure includes a modest inflation of the naively estimated error, multiplying it by a factor of 1.356 , and a constant correction applied to the naively estimated $m_{t}$, reducing it by $0.2755 \mathrm{GeV} / c^{2}$. After this mapping procedure our method produces an unbiased measurement of $m_{t}$ with accurately estimated errors.

But, even with this confidence, it would be helpful to understand in more detail the reasons for the 1.356 inflation factor of the naively estimated errors. So, we have carried out a large number of additional studies, attempting to enforce and relax our approximations and assumptions in a systematic way. In the limit that all of our assumptions are enforced in the events from which we construct pseudo-experiments, we expect a pull distribution with unit width. This can be arranged with parton-level events generated to enforce our assumptions, or with fully simulated events with very tight event selection cuts applied. As assumptions are broken, by allowing in a wider variety of events, the pull width should increase. If we relax our event selection beyond our default, for example, by allowing in events with five hard jets, the pull width should increase beyond even 1.356. And, indeed, this is what we observe.

As these pull studies were conducted at several different stages in our analysis, and for comparison with various other analyses, they use a few different pseudo-experiment 
methodologies. These studies use only $t \bar{t}$ events and use the signal-only likelihood (using $P_{t \bar{t}}$ but not $\left.P_{b a c k}\right)$. We use resampled pseudo-experiments to estimate the error on the width of the pull distributions, resampling each event up to a maximum of $\left[\left(N_{m c} / N_{p e}\right)^{2}\right] / 3$ times. Here $N_{m c}$ is the total number of events available and $N_{p e}$ is the number of events per pseudo-experiments. For fully simulated events the default event selection here is a lepton + jets pre-tagged event selection, similar to our default event selection but nor requiring a $b$ tag, and includes the $P_{t \bar{t}}$ cut to remove events that behave pathologically under our signal probability. This case will be the "default" case for these studies, and we observe the width of pull distributions in this case to be $1.440 \pm 0.021$.

For Tables 12.1 to 12.4 we construct pseudo-experiments with a Poisson mean of 78.2 events (about the expected number of events in $318 \mathrm{pb}^{-1}$, assuming a $t \bar{t}$ cross section of $8.0 \mathrm{pb}$, and with no $b$ tag requirement applied).

Table 12.1 shows the widths of pull distributions for various cases close to the parton-level events that we would expect to have unit pull widths. The first case uses specially generated PYTHIA $t \bar{t}$ events where radiation and hadronization have been turned off. To make these events compatible with our default $P_{t \bar{t}}$ we smear the quark energies with the same transfer function shape we then use to analyze the events. We expect these events to have unit pull widths and indeed they do. The next case uses events close to parton level but extracted from our default fully simulated (HERWIG) event samples. These events are taken from the so-called HEPG bank generated by HERWIG. They are approximately parton-level, but include the effects of radiation (certainly initial state, perhaps final-state also) and perhaps some residual effects of hadronization. The next two samples are variations of this HEPG sample. They are meant to explore how much of the 1.440 pull width with fully simulated events is due to energy uncertainty (for example imperfections in the transfer functions, or perhaps irreducible uncertainty in their application) and how much due to differences between the angles of jets and partons (which effect we choose not to model in $P_{t \bar{t}}$. Guaranteeing the correct energies, by taking them directly from the HEPG event, produces a result very close to unity, while the uncertainty in jet angles seems to be a more modest effect.

Table 12.2 shows the widths of pull distributions for various event selections related to jets. The first case is among the most restrictive selections that we have productively applied to a fully simulated sample. We require exactly four tight $\left(p_{T}>15, \eta<2.0\right.$ jets as usual, but also veto events with any loose jets $\left(p_{T}>8, \eta<2.0\right)$. We require the four 


\begin{tabular}{|c|c|c|c|}
\hline Sample & \# of Events & Pull Width & $\begin{array}{c}\text { Mean } \\
m_{t}\left(\mathrm{GeV} / c^{2}\right)\end{array}$ \\
\hline Parton level $\left(175 \mathrm{GeV} / c^{2}\right)$, TF smeared & 2986 & $1.022 \pm 0.033$ & $175.68 \pm 0.50$ \\
\hline $\begin{array}{c}\text { HEPG level from } \\
\text { default MC }\left(178 \mathrm{GeV} / c^{2}\right), \mathrm{TF} \text { smeared }\end{array}$ & 2956 & $1.138 \pm 0.039$ & $177.97 \pm 0.43$ \\
\hline $\begin{array}{c}\text { Jet angles from HEPG, } \\
\text { Jet energies from full simulation }\end{array}$ & 2951 & $1.118 \pm 0.037$ & $178.01 \pm 0.30$ \\
\hline $\begin{array}{c}\text { Jet angles from full simulation, } \\
\text { Jet energies TF smeared HEPG }\end{array}$ & 2954 & $1.043 \pm 0.038$ & $178.65 \pm 0.40$ \\
\hline
\end{tabular}

Table 12.1: Widths of pull distributions for various cases close to parton-level. The first line is from a custom-generated PYTHIA $175 \mathrm{GeV} / c^{2} t \bar{t}$ sample with radiation and hadronization turned off. It should satisfy most of the assumptions of our model. The second line uses HEPG level events from our default $t \bar{t}$ simulation $\left(178 \mathrm{GeV} / c^{2}\right.$ HERWIG) and includes, for example, the effect of hard gluonic initial state radiation - so, for example, the $P_{T}$ of the $t \bar{t}$ system is not 0 . The third and fourth samples are hybrids of HEPG level and fully simulated information. The third takes the angles for quarks from HEPG and their energies from full simulation. The fourth takes the jet angles from full simulation but for energies begins with the quark energies from HEPG and applies TF smearing.

tight jets to match the four partons from $t \bar{t}$ decay to within a cone of $\Delta R<0.15$. We also require the event to be an $e$ or $\mu+$ jets event with $\mathrm{MC}$ truth information. The one significant requirement that we have not applied, due to limited statistics, is to limit the $p_{T}$ of the initial state, to select events with little initial-state radiation. Even so, these events approach unit pull width. The other cases show how, as we relax our assumptions, the pull widths gradually approach the default case. We see that the single most effective cut is a tight matching requirement, which tends to guarantee angular correspondence between jets and partons, and probably also correlates with a generally parton-like event.

Table 12.3 shows the widths of pull distributions for various types of lepton information. We see that, while events with true $e$ 's have somewhat lower pull widths than $\mu$ 's or $\tau$ 's, using even the parton-level four-vector for the lepton has no large effect on the pull widths. As per our assumption in $P_{t \bar{t}}$, leptons are reasonably well measured objects. The one significant deviation in the pull widths is seen if we require at least one $\tau$ at parton level while allowing those di-lepton events which pass our event selection. This highlights the contribution of di-lepton events (where one of the jets, and the missing $E_{T}$, comes from $\tau$ 's or another non-parton-level source) which we do not expect to model well with our lepton + jets derived $P_{t \bar{t}}$.

A comparison without the $P_{t \bar{t}}$ cut is shown in Table 12.4. As we have previously 


\begin{tabular}{|c|c|c|c|}
\hline Sample & \# of Events & Pull Width & $\begin{array}{c}\text { Mean } \\
m_{t}\left(\mathrm{GeV} / c^{2}\right)\end{array}$ \\
\hline $\begin{array}{c}4 \text { tight jets, match 0.15, } \\
\text { ljets, no tau, no loose }\end{array}$ & 1568 & $1.081 \pm 0.042$ & $178.45 \pm 0.45$ \\
\hline 4 tight jets, match 0.15 & 2035 & $1.128 \pm 0.060$ & $178.37 \pm 0.51$ \\
\hline 4 tight jets, match 0.4 & 4220 & $1.188 \pm 0.029$ & $177.83 \pm 0.43$ \\
\hline 4 tight jets, no loose, ljets, no tau & 4069 & $1.298 \pm 0.035$ & $178.18 \pm 0.41$ \\
\hline 4 tight jets, no loose & 4652 & $1.303 \pm 0.028$ & $178.37 \pm 0.26$ \\
\hline 4 tight jets, ljet, no taus & 6450 & $1.407 \pm 0.022$ & $176.97 \pm 0.43$ \\
\hline 4 tight jets, ljets & 6896 & $1.405 \pm 0.021$ & $177.50 \pm 0.33$ \\
\hline 4 tight jets (default) & 7322 & $1.440 \pm 0.021$ & $177.77 \pm 0.35$ \\
\hline
\end{tabular}

Table 12.2: Widths of pull distributions for various event selections related to jets. The first line is for a rather strict event selection. We require the four tight jets to match the four partons within a cone of $\Delta R<0.15$, we require $e$ or $\mu+$ jets events identified from $\mathrm{MC}$ truth and we veto the event if there are any loose jets $\left(P_{T}>8, \eta<2.0\right)$. The second line is our default selection with only the $\Delta R<0.15$ matching requirement added. The third line is with a looser, $\Delta R<0.4$, matching requirement. The fourth line makes no matching requirement, but requires e or $\mu+$ jets and vetoes loose jets. The fifth line only vetoes loose jets. The sixth line allows loose jets, but requires e or $\mu+$ jets. The sixth line requires e, $\mu$, or $\tau+$ jets (eliminating dilepton and all-hadronic events). The last line is for our default event selection.

\begin{tabular}{|c|c|c|c|}
\hline Sample & \# of Events & Pull Width & $\begin{array}{c}\text { Mean } \\
m_{t}\left(\mathrm{GeV} / c^{2}\right)\end{array}$ \\
\hline 4 tight jets, ljets & 6896 & $1.405 \pm 0.021$ & $177.50 \pm 0.33$ \\
\hline 4 tight jets, ljets, HEPG e & 4013 & $1.396 \pm 0.036$ & $177.62 \pm 0.43$ \\
\hline 4 tight jets, ljets, HEPG mu & 2437 & $1.447 \pm 0.057$ & $177.12 \pm 0.72$ \\
\hline 4 tight jets, ljets, HEPG tau & 446 & $1.466 \pm 0.31$ & $178.36 \pm 0.99$ \\
\hline HEPG lepton, reconstructed jets & 2910 & $1.396 \pm 0.060$ & $177.25 \pm 0.34$ \\
\hline 4 tight jets (default) & 7322 & $1.440 \pm 0.021$ & $177.77 \pm 0.35$ \\
\hline 4 tight jets, one or more taus & 679 & $1.81 \pm 0.26$ & $178.2 \pm 1.2$ \\
\hline
\end{tabular}

Table 12.3: Widths of pull distributions for various lepton-related event selections. The first line is our default event selection with the additional requirement that the event be lepton $(e, \mu$, or $\tau)+$ jets at MC truth (i.e. HEPG) level. The next three lines are exclusive subsets of the first line, taking the lepton identification from HEPG level but using the reconstructed four-momentum. The fourth line uses the four-momentum from the HEPG bank. The fifth line is our default, included for comparison. And, the last line uses fourvectors from fully reconstructed simulation, but uses HEPG particle identification to require one or more $\tau$ 's (intentionally allowing what dilepton events pass our event selection). All lines except the fifth are subsets of the same 7500-event sample. The fifth is based on the first 3000-event subset of this. 


\begin{tabular}{|c|c|c|c|}
\hline Sample & \# of Events & Pull Width & $\begin{array}{c}\text { Mean } \\
m_{t}\left(\mathrm{GeV} / c^{2}\right)\end{array}$ \\
\hline $\begin{array}{c}\text { parton level }\left(175 \mathrm{GeV} / c^{2}\right), \\
10 \% \text { Gaussian smeared }\end{array}$ & 2976 & $1.091 \pm 0.039$ & $175.7 \pm 0.16$ \\
\hline parton level $\left(175 \mathrm{GeV} / c^{2}\right), \mathrm{TF}$ smeared & 2995 & $1.107 \pm 0.040$ & $176.2 \pm 0.54$ \\
\hline 4 tight jets (default) & 7468 & $1.653 \pm 0.025$ & $178.7 \pm 0.40$ \\
\hline
\end{tabular}

Table 12.4: Pull distributions for selected samples without the $P_{t \bar{t}}$ cut. Pulls are wider than the case with the $P_{t \bar{t}}$ cut by a relative factor of about $10 \%$.

discussed, removing this cut allows events to enter the sample that behave pathologically under the $P_{t \bar{t}}$ probability. These events affect the mean $m_{t}$ value and also increase the pull widths by a relative factor of about $10 \%$. Events where the jets are smeared by a Gaussian of $10 \%$ width behave in about the same way as events smeared by our realistic transfer function.

Tables 12.5 to 12.10 were constructed as a part of a collaboration-wide effort for matrix element-based (and other) top mass measurements at CDF to understand issues related to pull distributions. So, pseudo-experiments were constructed with common methodology for all measurements: somewhat different methodology than we have used otherwise. For these studies we construct pseudo-experiments with exactly 30 (not Poisson fluctuated) $t \bar{t}$ events from the same default HERWIG sample $\left(m_{t}=178 \mathrm{GeV} / c^{2}\right.$ that we normally use. We apply the lepton + jets pre-tagged event selection, check the MC truth information (i.e. HEPG) to require the lepton + jets decay channel, and require exactly four tight jets (unless otherwise specified). We also match $b$ jets and hadronic $W$ jets to within a cone of $\Delta R<0.4$ with the appropriate partons.

Table 12.5 shows some simple checks with HEPG parton-level events smeared where parton energies have been smeared with our realistic transfer function. We further split events into categories depending on the $p_{T}$ of the $t \bar{t}$ system, computed from MC truth parton-level quantities. Statistics our limited because of the many cuts, but there seems to be a trend. When we enforce low $p_{T}$, consistent with our assumption of no $p_{T}$ we see pulls consistent with unity. When we select events with large $p_{T}$ kicks from initial-state radiation we see larger pull widths.

Table 12.6 checks the effects from leptons. We see no large differences between measured and HEPG leptons, between $e$ 's and $\mu$ 's, nor between forward (PHX) and central (CEM) electrons. 


\begin{tabular}{|c|c|c|c|}
\hline Sample & \# of Events & Pull Width & $\begin{array}{c}\text { Mean } \\
m_{t}\left(\mathrm{GeV} / c^{2}\right)\end{array}$ \\
\hline $\begin{array}{c}\text { HEPG level from } \\
\text { default MC }\left(178 \mathrm{GeV} / c^{2}\right), \mathrm{TF} \text { smeared }\end{array}$ & 1611 & $1.142 \pm 0.027$ & $178.0 \pm 0.38$ \\
\hline $\begin{array}{c}\text { HEPG level from } \\
\text { default MC }\left(178 \mathrm{GeV} / c^{2}\right), \mathrm{TF} \text { smeared, } \\
p_{T}^{t \bar{t}}<10 \mathrm{GeV} / c^{2}\end{array}$ & 687 & $0.997 \pm 0.064$ & $177.4 \pm 0.50$ \\
\hline $\begin{array}{c}\mathrm{HEPG} \text { level from } \\
\text { default MC }\left(178 \mathrm{GeV} / c^{2}\right), \mathrm{TF} \text { smeared, } \\
10 \mathrm{GeV} / c<p_{T}^{t \bar{t}}<20 \mathrm{GeV} / c\end{array}$ & 513 & & \\
\hline $\begin{array}{c}\mathrm{HEPG} \text { level from } \\
\text { default } \mathrm{MC}\left(178 \mathrm{GeV} / c^{2}\right), \mathrm{TF} \text { smeared, } \\
p_{T}^{t \bar{t}}>20 \mathrm{GeV} / c\end{array}$ & & & \\
\hline
\end{tabular}

Table 12.5: Widths of pull distributions for parton-level (HEPG) events where the parton energies have been smeared with realistic transfer functions, for various amounts of initial-state radiation $\left(t \bar{t}\right.$ system $\left.p_{T}\right)$. Statistics are limited because of tight event selection requirements, but the trend is that when there is little radiation (as assumed in our $P_{t \bar{t}}$ calculation) the pull widths are better behaved.

\begin{tabular}{|c|c|c|c|}
\hline Sample & \# of Events & Pull Width & $\begin{array}{c}\text { Mean } \\
m_{t}\left(\mathrm{GeV} / c^{2}\right)\end{array}$ \\
\hline$e$ from HEPG - select on CEM & 837 & $1.189 \pm 0.057$ & $178.1 \pm 0.55$ \\
\hline$\mu$ from HEPG - select on CMUP, CMX & 567 & $1.211 \pm 0.064$ & $177.3 \pm 0.74$ \\
\hline$e$ measured - select on CEM & 1990 & $1.177 \pm 0.023$ & $178.0 \pm 0.37$ \\
\hline$\mu$ from measured - select on CMUP, CMX & 1493 & $1.229 \pm 0.031$ & $177.2 \pm 0.45$ \\
\hline$e$ measured - select on CEM+PHX & 2470 & $1.182 \pm 0.020$ & $177.6 \pm 0.33$ \\
\hline
\end{tabular}

Table 12.6: Widths of pull distributions for various types of lepton information. For the first two lines we use measured jet quantities and HEPG information for the $e$ and $\mu$. We also show the $e$ and $\mu$ channels separately. For the second two lines we use measured jet and measured lepton quantities. We also check for differences between forward (PHX) and central (CEM) electrons in the last line. 


\begin{tabular}{|c|c|c|c|}
\hline Sample & \# of Events & Pull Width & $\begin{array}{c}\text { Mean } \\
m_{t}\left(\mathrm{GeV} / c^{2}\right)\end{array}$ \\
\hline 4 jets matched 0.4 & 3963 & $1.191 \pm 0.012$ & $177.5 \pm 0.26$ \\
\hline 4 jets matched 0.4, no loose & 2928 & $1.177 \pm 0.016$ & $177.8 \pm 0 . x x$ \\
\hline 4 jets matched 0.15, no loose & 1568 & $1.145 \pm 0.031$ & $178.4 \pm 0.37$ \\
\hline 4 jets no matching, no loose & 4069 & $1.312 \pm 0.012$ & $177.7 \pm 0.30$ \\
\hline 4 jets no matching & 6450 & $1.470 \pm 0.008$ & $177.2 \pm 0.28$ \\
\hline
\end{tabular}

Table 12.7: Widths of pull distributions for various types of matching requirements. This is one way to check for effects from initial- and final-state radiation jets.

\begin{tabular}{|c|c|c|c|}
\hline Sample & \# of Events & Pull Width & $\begin{array}{c}\text { Mean } \\
m_{t}\left(\mathrm{GeV} / c^{2}\right)\end{array}$ \\
\hline $\begin{array}{c}4 \text { jets matched } 0.4 \\
p_{T}^{t \bar{t}}<10 \mathrm{GeV} / c\end{array}$ & 7457 & $1.168 \pm 0.006$ & $176.4 \pm 0.19$ \\
\hline $\begin{array}{c}4 \text { jets matched } 0.4 \\
10 \mathrm{GeV} / c<p_{T}^{t \bar{t}}<20 \mathrm{GeV} / c\end{array}$ & 7449 & $1.214 \pm 0.006$ & $177.7 \pm 0.20$ \\
\hline $\begin{array}{c}4 \text { jets matched } 0.4 \\
p_{T}^{t \bar{t}}>20 \mathrm{GeV} / c\end{array}$ & 6957 & $1.295 \pm 0.007$ & $179.3 \pm 0.23$ \\
\hline
\end{tabular}

Table 12.8: Widths of pull distributions in different bins of $p_{T}^{t \bar{t}}$ to check for radiation effects. This is similar to the case for transfer function-smeared partons in Table 12.5.

Tables 12.7 through 12.9 study the effects from extra jets, generally caused by initial- and final-state radiation. Table 12.7 looks at various matching requirements while Table 12.8 revisits the binned studies of $p_{T}^{t \bar{t}}$ but now with fully simulated events. Conclusions in both cases are the same as with previous similar studies. Table 12.9 considers various matching scenarios for events with four or more tight jets. Here we also consider the case of requiring separately matches for the $W$ jets and the $b$ jets. The theme is much the same that we have seen so far, enforcing our assumptions, especially better agreement between jets and partons, leads to pull distributions closer to unity. Here we see also that four events with more than four tight jets the pull widths are even worse than for the default case. These events are clearly not well-modelled by the leading order $t \bar{t}$ matrix element.

Table 12.10 checks for effects from jet combinatorics. We consider all twelve parton-jet combinations, or only the correct combination, the case with one or the other $b$ tagged, and the case with both $b$ 's tagged. We find that using only the correct combination reduces the pull width.

One summary of our pull width studies is shown in Figure 12.11. From our default 


\begin{tabular}{|c|c|c|c|}
\hline Sample & \# of Events & Pull Width & $\begin{array}{c}\text { Mean } \\
m_{t}\left(\mathrm{GeV} / c^{2}\right)\end{array}$ \\
\hline 4 jets matched 0.4 & 4010 & $1.346 \pm 0.013$ & $178.6 \pm 0.32$ \\
\hline 4 jets matched 0.4, no loose & 2917 & $1.234 \pm 0.016$ & $178.9 \pm 0 . x x$ \\
\hline 4 jets matched 0.15, no loose & 1419 & $1.205 \pm 0.032$ & $179.9 \pm 0.45$ \\
\hline no W jets matching & 4841 & $1.442 \pm 0.012$ & $178.8 \pm 0.33$ \\
\hline no b jets matching & 4724 & $1.421 \pm 0.012$ & $178.3 \pm 0.32$ \\
\hline no matching & 6401 & $1.594 \pm 0.010$ & $178.3 \pm 0.32$ \\
\hline
\end{tabular}

Table 12.9: Widths of pull distributions for events with four or more tight jets. We consider also the case of separately requiring matching for $W$ and $b$ jets. The case with no matching shows that when we relax the event selection requirements beyond our default, by allowing more than four tight jets, the pull widths do indeed grow even larger.

\begin{tabular}{|c|c|c|c|}
\hline Sample & \# of Events & Pull Width & $\begin{array}{c}\text { Mean } \\
m_{t}\left(\mathrm{GeV} / c^{2}\right)\end{array}$ \\
\hline 6 combinations, leptonic b & 5179 & $1.198 \pm 0.008$ & $177.7 \pm 0.22$ \\
\hline 6 combinations, hadronic b & 5164 & $1.17 \pm 0.01$ & $178.1 \pm 0.22$ \\
\hline 2 combinations & 5018 & $1.145 \pm 0.009$ & $178.1 \pm 0.19$ \\
\hline Right combination & 4621 & $1.116 \pm 0.009$ & $177.9 \pm 0.18$ \\
\hline All combinations & 3963 & $1.191 \pm 0.012$ & $177.5 \pm 0.26$ \\
\hline
\end{tabular}

Table 12.10: Widths of pull distributions for various jet combinations. We use the measured lepton information and then consider several scenarios. The first line assumes we have correctly tagged the leptonic $b$ and considers the remaining six combinatoric possibilities. Similarly the second line assumes knowledge of the identity of the hadronic $b$. The third line assumes two $b$ tags and just considers the case where we swap the two $b$ jets. Finally we consider using only the correct combination, and using all twelve combinations (our default). 


\begin{tabular}{|c|c|c|}
\hline Sample & Pull Width & Table \\
\hline$\geq 4$ tight jets & $1.594 \pm 0.010$ & 12.9 \\
\hline Default (exactly 4 tight jets) & $1.440 \pm 0.021$ & 12.2 \\
\hline 4 tight jets, no loose jets & $1.303 \pm 0.028$ & 12.2 \\
\hline 4 tight jets, no loose jets & & \\
with tight parton-jet matching $(\Delta R<0.15)$ & $1.128 \pm 0.060$ & 12.2 \\
\hline HEPG-level events & $1.142 \pm 0.027$ & 12.5 \\
\hline HEPG-level events with $p_{T}^{t t}<10 \mathrm{GeV} / c^{2}$ & $0.997 \pm 0.064$ & 12.5 \\
\hline Custom parton-level events & $1.022 \pm 0.033$ & 12.1 \\
\hline
\end{tabular}

Table 12.11: One summary of our pull width studies. The entries in this table are drawn from various tables in this chapter, and are not necessarily generated with the same methodology. So, this table should be taken as a sketch of an argument only, for further details refer to the individual tables. Overall the trend here is clear. As we enforce the assumptions we make in calculating $P_{t \bar{t}}$, either with fully simulated events, parton-level events, or custom-generated clean parton events, our pull widths approach unity. As we break those assumptions the pull widths increase.

pull width of 1.44 we see that further violating our assumptions, by allowing in a fifth hard jet, increases the pull width to 1.59. A pull width of approximately 1.15 can be obtained either with transfer function smeared HEPG events (parton-level events that underpin the fully simulated events) or from fully simulated events with tight event selection cuts applied. In general it seems that the pull widths are most affected by radiation and other additional calorimeter activity. These violate our assumptions that there is no radiation, that the jet angles correspond to the parton angles, that the $p_{T}$ of the $t \bar{t}$ system is zero, and that we can calculate the neutrino $p_{T}$ as the opposite of the sum of the other five $p_{T}$ 's (four quarks and a lepton) in our matrix element. Releasing the selection and allowing more radiation increases the pull widths. Requiring only four tight jets and no loose jets help reduce the pull widths. Also, we find the correct combination gives a pull width smaller than for all combinations. We observe that we are not sensitive to lepton type or mismeasurement.

One possible accounting of the blame for our 1.44 pull width might be as follow: $3 \%$ of our excess pull width is due to dilepton, all-hadronic, and $\tau$ events, $11 \%$ is due to loose jets, $22 \%$ is due to other physical effects that spoil our matching, and $8 \%$ is due to residual effects that don't significantly affect our matching, such as initial-state-radiation. 


\subsection{Results of Blind Samples}

This entire analysis has been conducted in the spirit of a blind analysis. Many of our calibrations at CDF rely, of course, on data samples (e.g. $\gamma$-jet balancing as a cross-check of the jet energy scale calibration). And, our background estimates are data driven as well. But, we did not run our $m_{t}$ fitting machinery on the $t \bar{t}$ event sample until the machinery was tested and calibrated on fully simulated events, approved by the CDF collaboration, and frozen. Only then did we look at the final sixty-three event lepton + jets dataset and unveil our $m_{t}$ result. The measurement was not, perhaps, blind in the strictest sense since other analyses (probably even other $m_{t}$ analyses) did look at the data sense in advance of our measurement. So, we knew there were no major problems with the data set.

As an additional confidence-building measure blind samples of fully simulated $t \bar{t}$ Monte Carlo were produced by the coordinators of the top physics group at CDF. Five different samples were prepared with their identities and masses concealed. We have constructed pseudo-experiments from these blinded $t \bar{t}$ samples and passed them through our analysis machinery in the same fashion as the data. For historical reasons, we construct the pseudo-experiments with 50.58 Poisson-mean signal events and 12.42 Poisson-mean background events. The parton-showering code used for each sample, and the residual (true mass subtracted from the fitted mass) were reported, but not the mass of the sample itself. The plotted residuals can be found in Figure 12.1. The 4th and 5th samples are from PYTHIA, which in our systematic studies seemed to be lower than our HERWIG default. Fitting the five residuals to a constant of $0 \mathrm{GeV} / c^{2}$ gives a $\chi^{2}$ probability of $23 \%$. So, the results from the blinded samples are consistent with showing no bias. 


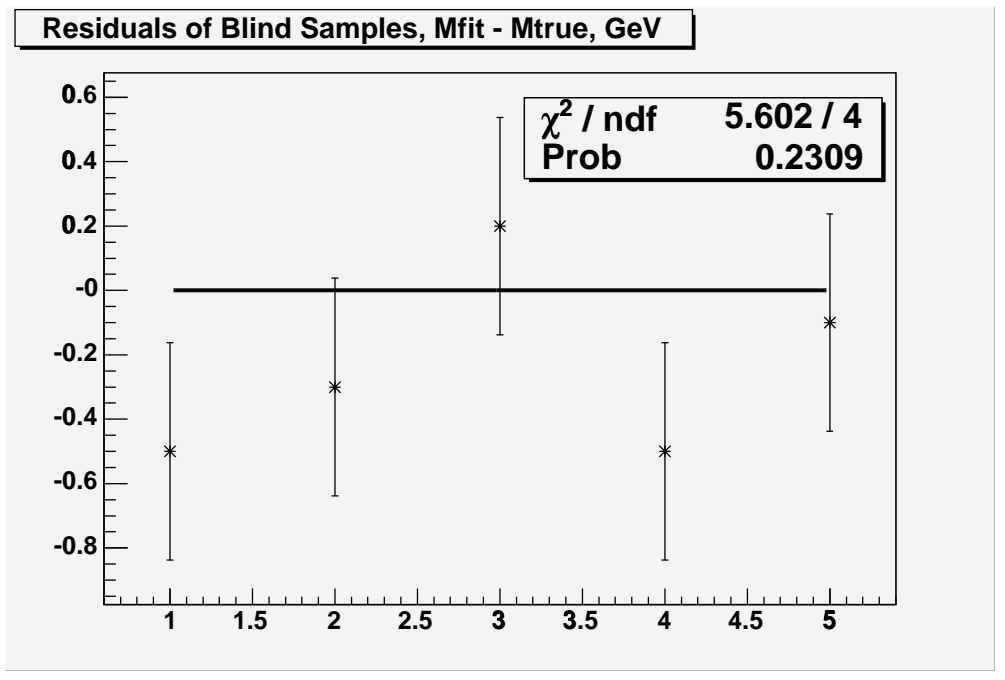

Figure 12.1: Residuals (in $\mathrm{GeV} / c^{2}$ ) of blinded $t \bar{t}$ samples plotted vs. sample number. Five samples of unknown $m_{t}$ and Monte Carlo generator were analyzed with our default machinery. The $m_{t}$ residuals, true mass subtracted from fitted mass, were revealed as well as the identity of the generator. The 4th and 5th samples are from PYTHIA, which in our systematic studies seemed to be lower than our HERWIG default. Fitting the five residuals to a constant of $0 \mathrm{GeV} / c^{2}$ gives a $\chi^{2}$ probability of $23 \%$. So, the results from the blinded samples are consistent with showing no bias. 


\section{Chapter 13}

\section{Conclusions}

We have performed a measurement of the mass of the top quark. $m_{t}$ is directly related to the top quark Yukawa coupling, a fundamental parameter of the Standard Model. And, through the program of precision electroweak physics, $m_{t}$ is intertwined with the mass of the putative Higgs boson, and will aid in the further development or overthrow of the Standard Model. Our event sample is selected from proton-antiproton collisions, at 1.96 $\mathrm{TeV}$ center-of-mass energy, observed with the CDF detector at Fermilab's Tevatron. We have considered a $318 \mathrm{pb}^{-1}$ dataset collected between March 2002 and August 2004. Our event selection requires one energetic lepton, large missing transverse energy, exactly four energetic jets, and at least one displaced vertex $b$ tag. Our analysis uses leading-order $t \bar{t}$ and background matrix elements along with parameterized parton showering to construct event-by-event likelihoods as a function of top quark mass. From the 63 events observed with the $318 \mathrm{pb}^{-1}$ dataset we extract a top quark mass of $172.0 \pm 2.6$ (stat) \pm 3.3 (syst) $\mathrm{GeV} / c^{2}$ from the joint likelihood. The mean expected statistical uncertainty is $3.2 \mathrm{GeV} / c^{2}$ for $m_{t}=178 \mathrm{GeV} / c^{2}$ and $3.1 \mathrm{GeV} / c^{2}$ for $m_{t}=172.5 \mathrm{GeV} / c^{2}$. The systematic error is dominated by the uncertainty of the jet energy scale.

Our method stands in the tradition of other top mass analyses performed with matrix element techniques such as the Dynamical Likelihood Method $m_{t}$ analysis at CDF [27] and the much heralded analysis of Tevatron Run I data at D0 [23]. At CDF it is the first matrix element method to use a background matrix element.

Of course, there are many possible improvements that could be made to this analysis, and will be considered for future iterations. Probably the most significant of these, in in situ jet energy scale calibration using the known mass of the $W$ decaying into jets, has 
already been incorporated into the next version of this analysis. But, there remain a large number of possible improvements. One which should be fairly straightforward is to address the under-utilization of $b$ tagging in combinatorics. For historical reasons this analysis has always considered all twelve possible jet-parton combinations (twelve because $P_{t \bar{t}}$ has already been symmetrized under exchange of the light quarks). But, when we have one $b$ tag we need only consider six, and only two when two $b$ tags are available. Incorporating this should improve the $m_{t}$ resolution by several percent. Another consideration that suggests a technical shortcoming in the current analysis is the $P_{t \bar{t}}$ cut. Probably there is some more elegant way to eliminate the pathology that this cut was designed to address, and perhaps more can be learned about the method by studying this symptom in greater detail. One final improvement, which should be relatively easy to implement, is a subdivision of the event sample. We know that the signal and background expectations are different in the sub-samples with one and two $b$ tags, and we should be able to modestly improve the $m_{t}$ resolution by making a measurement separately in these two samples, and then combining them. This will become more important as other sub-samples are added.

Another category of possible improvements is those that extend our data sample, increasing our acceptance. Our choice in which sample to begin with (lepton + jets, relatively tight event selection, exactly four jets, at least one $b$ tag, etc.) was motivated by those events which we expect to have the most information about $m_{t}$ and be the most consistent with the assumptions of our method. But, a more precise measurement of $m_{t}$ can probably be obtained by moving beyond this relatively limited sample of events. One obvious step is to include events with no $b$ tag, though they have a larger fraction of background events, and the background normalization is less well understood. We could also incorporate other methods of identifying $b$ jets such as jets tagged via soft leptons, or even make use of probabilistic $b$ taggers which have a natural affinity for a likelihood-based measurement such as ours. We could consider using electrons, muons, and jets in the more forward regions of our detector. We might also consider using softer jets, as other $m_{t}$ measurements have done. Events with more than four jets also come to mind, but these are considerably less trivial to incorporate. We might consider a next-to-leading order matrix element, perhaps necessarily with gluonic transfer functions as well as our light and $b$ transfer functions. But, this is daunting from a computational perspective as well as an intellectual one. Expanding the acceptance of our sample may improve our $m_{t}$ resolution and would certainly provide useful cross-checks of this measurement. 
The final category of improvements that I will discuss here relate to probing our assumptions, which would likely have the added effect of improving our understanding of the widths of our pull distributions. If we chose to enforce our assumptions more strictly, or to adopt methods with less restrictive assumptions, we would simply move the width closer to unity. Our most thoroughly broken assumption seems to be the connection between jets and partons. This could perhaps be addressed by incorporating an angular transfer function. Such a function would include a probability density that the angular positions of jets (or perhaps the opening angle between pairs of jets) differ from the parton case. Presumably this would incorporate additional integrations, which is computationally challenging. On a somewhat related tack, one could consider further extending the transfer functions. We have seen no evidence for a necessary $\eta$-dependence in our current functions, nor for an obvious need for background-specific or gluonic transfer functions. But, these studies could be reconsidered. Or, we could choose to be less reliant on the CDF collaboration-wide generic jet corrections and incorporate more of the detector effects (and their dependence on $\eta$, the electromagnetic fraction of the jet, etc.) into our transfer functions. Without extending our transfer functions, we might consider different possible parameterizations, or even nonparametric approach without an explicit fit. We could also consider modelling additional background processes with matrix elements, improving our modelling of the difficult QCD background, or incorporating a $g g$ matrix element in $P_{t \bar{t}}$. We have also seen the effect of our assumption that the $t \bar{t}$ system has no $p_{T}$. This can be addressed by allowing for such $p_{T}$, and the next version of this analysis in fact incorporates two additional integrals to make such an allowance.

To this date our measurement is third most precise $m_{t}$ measurement announced on a dataset of approximately $318 \mathrm{pb}^{-1}$. The single most precise measurement in the world, on any dataset, is the descendant of this analysis, and makes the important addition of an in situ jet energy scale calibration based on the mass of hadronically decaying $W$ bosons in the top quark decays. The latest measurements at CDF are always available electronically [21] as is the case for D0. So far in Run II D0's reported results have been less precise than those at CDF, but when measurements quote only their measured error, rather than also quoting their expected error, it is difficult to compare a priori. In the paragraphs above we have listed a host of possible improvements. With the most important of these, the in situ jet energy calibration, already implemented, contemporary $m_{t}$ measurements have become statistically dominated. So, improving the statistical resolution of the method, with ideas 
such as those outlined above, will be helpful. Of course, while the Tevatron continues to run, luminosity alone will improve the $m_{t}$ result, as shown in Figure 2.14. Another step, for those who continue active development of $m_{t}$ analyses at the Tevatron, will be to address the wide range of systematics (see Table 11.9) that compete after the jet energy scale. At the Large Hadron Collider the top mass measurement will be an important calibration of the jet energy scale and an important test of the new detectors. It will be interesting to see if precision $m_{t}$ measurements at the Large Hadron Collider ever pass from the Tevatron, or whether further improvements must await the International Linear Collider. 


\section{Bibliography}

[1] J. J. Thomson, "Cathode Rays,", Philosophical Magazine 44, 293 (1897).

[2] K. Kodama et al. [DONUT Collaboration], "Observation of Tau Neutrino Interactions," Phys. Lett. B 504, 218 (2001) [arXiv:hep-ex/0012035].

[3] F. Abe et al. [CDF Collaboration], "Evidence for Top Quark Production in anti-p p collisions at $\mathrm{s}^{* *}(1 / 2)=1.8-\mathrm{TeV}, "$ Phys. Rev. D 50, 2966 (1994).

[4] F. Abe et al. [CDF Collaboration], "Evidence for Top Quark Production in anti-p p collisions at s** $(1 / 2)=1.8-\mathrm{TeV}, "$ Phys. Rev. Lett. 73, 225 (1994) [arXiv:hep-ex/9405005].

[5] F. Abe et al. [CDF Collaboration], "Observation of Top Quark Production in anti-p p Collisions with the Collider Detector at Fermilab," Phys. Rev. Lett. 74, 2626 (1995) [arXiv:hep-ex/9503002].

[6] S. Abachi et al. [D0 Collaboration], "Observation of the Top Quark," Phys. Rev. Lett. 74, 2632 (1995) [arXiv:hep-ex/9503003].

[7] D. Chakraborty, J. Konigsberg and D. L. Rainwater, "Top-Quark Physics," Ann. Rev. Nucl. Part. Sci. 53, 301 (2003) [arXiv:hep-ph/0303092].

[8] S. Willenbrock, "Studying the top quark," Rev. Mod. Phys. 72, 1141 (2000) [arXiv:hepph/0008189].

[9] S. Willenbrock, "Thinking About Top within the Standard Model," arXiv:hep$\mathrm{ph} / 9905498$.

[10] E. H. Simmons, "Thinking About Top: Looking Outside The Standard Model," arXiv:hep-ph/9908511. 
[11] S. Willenbrock, "The Standard Model and the Top Quark," arXiv:hep-ph/0211067.

[12] M. Beneke et al., "Top Quark Physics," arXiv:hep-ph/0003033. Especially useful for discussion of top at LHC.

[13] E. W. N. Glover et al., "Top Quark Physics at Colliders," Acta Phys. Polon. B 35, 2671 (2004) [arXiv:hep-ph/0410110]. Especially useful for discussion of top at ILC.

[14] M. Cacciari, S. Frixione, M. L. Mangano, P. Nason and G. Ridolfi, "The t anti-t crosssection at $1.8-\mathrm{TeV}$ and $1.96-\mathrm{TeV}$ : A study of the systematics due to parton densities and scale dependence," JHEP 0404, 068 (2004) [arXiv:hep-ph/0303085].

[15] N. Kidonakis and R. Vogt, "Next-to-next-to-leading order soft-gluon corrections in top quark hadroproduction," Phys. Rev. D 68, 114014 (2003) [arXiv:hep-ph/0308222].

[16] R. Barate et al. [LEP Working Group for Higgs boson searches], "Search for the Standard model Higgs boson at LEP," Phys. Lett. B 565, 61 (2003) [arXiv:hep-ex/0306033].

[17] S. Eidelman et al. [Particle Data Group], "Review of particle physics," Phys. Lett. B 592, 1 (2004).

[18] The LEP Collaborations ALEPH, DELPHI, L3, OPAL, and the LEP Electroweak Working Group. "A Combination of Preliminary Electroweak Measurements and Constraints on the Standard Model: Prepared from Contributions of the LEP Experiments to the 2005 Summer Conferences," arXiv:hep-ex/0511027. Latest results available at http://lepewwg.web.cern.ch/LEPEWWG/.

[19] S. Heinemeyer, W. Hollik and G. Weiglein, "Electroweak precision observables in the minimal supersymmetric standard model,” Phys. Rept. 425, 265 (2006) [arXiv:hep$\mathrm{ph} / 0412214]$. arXiv.org version is somewhat out of date.

[20] C. T. Hill, "Topcolor assisted technicolor," Phys. Lett. B 345, 483 (1995) [arXiv:hep$\mathrm{ph} / 9411426]$.

C. T. Hill, "Topcolor: Top quark condensation in a gauge extension of the standard Phys. Lett. B 266, 419 (1991).

D. Kominis, "Topcolor: A dynamical approach to top-quark mass generation," arXiv:hep-ph/9605272. 
[21] The most recent top physics results, including top mass results, are available from D0 at http://www-d0.fnal.gov/Run2Physics/WWW/results/top.htm and from CDF at http://www-cdf.fnal.gov/physics/new/top/top.html.

[22] The CDF Collaboration, the D0 Collaboration, and the Tevatron Electroweak Working Group, "Combination of CDF and D0 Results on the Top-Quark mass," arXiv:hepex/0507091.

[23] V. M. Abazov et al. [D0 Collaboration], "A Precision Measurement of the Mass of the Top Quark," Nature 429, 638 (2004) [arXiv:hep-ex/0406031].

V. M. Abazov et al. [D0 Collaboration], "Helicity of the W boson in Lepton + Jets $\mathrm{t}$ anti-t events," Phys. Lett. B 617, 1 (2005) [arXiv:hep-ex/0404040].

[24] R. H. Dalitz and G. R. Goldstein, "Test of Analysis Method for Top-Antitop Production and Decay Events," Proc. Roy. Soc. Lond. A 455, 2803 (1999) [arXiv:hep-ph/9802249].

[25] K. Kondo, "Dynamical Likelihood Method For Reconstruction Of Events With Missing Momentum. 1: Method And Toy Models," J. Phys. Soc. Jap. 57, 4126 (1988).

K. Kondo, "Dynamical Likelihood Method for Reconstruction of Events with Missing Momentum. 2: Mass Spectra for $2 \rightarrow 2$ processes," J. Phys. Soc. Jap. 60, 836 (1991).

[26] A. Abulencia et al. [CDF Collaboration], "Precision top quark mass measurement in the lepton + jets topology in p anti-p collisions at $\mathrm{s}^{* *}(1 / 2)=1.96-\mathrm{TeV}$," Phys. Rev. Lett. 96, 022004 (2006) [arXiv:hep-ex/0510049].

[27] A. Abulencia et al. [CDF Collaboration], "Measurement of the Top Quark Mass with the Dynamical Likelihood Method using Lepton plus Jets Events with b-tags in p antip collisions at s** $(1 / 2)=1.96-\mathrm{TeV}, "$ arXiv:hep-ex/0512009. Submitted to Phys. Rev. D December 5, 2005.

[28] [CDF Collaboration], "Top Quark Mass Measurement from Dilepton Events at CDF II," arXiv:hep-ex/0512070. Submitted to Phys. Rev. Lett. December 22, 2005.

[29] A. Abulencia et al. [CDF Collaboration], "Top quark mass measurement using the template method in the lepton + jets channel at CDF II," arXiv:hep-ex/0510048. Phys. Rev. D 73, 032003 (2006) (35 pages) 
[30] Projections for CDF Top Mass Uncertainty in Run 2 - plot from March 2006 and taken from http://www-cdf.fnal.gov/physics/new/top/2006/CDFExtrap/

[31] Operations Department, Accelerator Division, Fermi National Accelerator Laboratory. "Rookie Books". http://www-bdnew.fnal.gov/operations/rookie_books/rbooks.html.

[32] D. Mohl, G. Petrucci, L. Thorndahl and S. Van Der Meer, "Physics And Technique Of Stochastic Cooling," Phys. Rept. 58, 73 (1980).

[33] F. Abe et al. [CDF Collaboration], "The CDF Detector: An Overview," Nucl. Instrum. Meth. A 271, 387 (1988).

[34] R. Blair et al. [CDF-II Collaboration], "The CDF-II detector: Technical design report," FERMILAB-PUB-96-390-E

[35] D. Acosta et al. [CDF Collaboration], "Measurement of the $\mathrm{J} / \mathrm{psi}$ meson and b-hadron production cross sections in $\mathrm{p}$ anti-p collisions at $\mathrm{s}^{* *}(1 / 2)=1960-\mathrm{GeV}$," Phys. Rev. D 71, 032001 (2005) [arXiv:hep-ex/0412071].

[36] A. Sill [CDF Collaboration], "CDF Run II silicon tracking projects," Nucl. Instrum. Meth. A 447, 1 (2000).

[37] L. Vacavant, "GEANT Description of the CDF Inner Tracker Passive Material", CDF Internal Note 5825 (2002).

[38] T. Affolder et al. [CDF Collaboration], "CDF Central Outer Tracker," Nucl. Instrum. Meth. A 526, 249 (2004).

[39] D. Acosta et al. [CDF-II Collaboration], "A Time-of-Flight Detector in CDF-II," Nucl. Instrum. Meth. A 518, 605 (2004).

[40] L. Balka et al. [CDF Collaboration], "The CDF Central Electromagnetic Calorimeter," Nucl. Instrum. Meth. A 267, 272 (1988).

[41] S. Bertolucci et al. [CDF Collaboration], "The CDF Central And Endwall Hadron Calorimeter," Nucl. Instrum. Meth. A 267, 301 (1988).

[42] M. G. Albrow et al. [CDF Collaboration], "The CDF plug upgrade electromagnetic calorimeter: test beam results," Nucl. Instrum. Meth. A 480, 524 (2002). 
[43] G. Ascoli et al., "CDF Central Muon Detector," Nucl. Instrum. Meth. A 268, 33 (1988).

[44] A. Artikov et al., "Design and construction of new central and forward muon counters for CDF II," Nucl. Instrum. Meth. A 538, 358 (2005) [arXiv:physics/0403079].

[45] D. Acosta et al. [CDF Collaboration], "The CDF Cherenkov luminosity monitor," Nucl. Instrum. Meth. A 461, 540 (2001).

[46] G. Corcella et al., "HERWIG 6: An event generator for Hadron Emission Reactions With Interfering Gluons (including supersymmetric processes)," JHEP 0101, 010 (2001) [arXiv:hep-ph/0011363].

[47] T. Sjostrand, P. Eden, C. Friberg, L. Lonnblad, G. Miu, S. Mrenna and E. Norrbin, "High-energy-physics event generation with PYTHIA 6.1," Comput. Phys. Commun. 135, 238 (2001) [arXiv:hep-ph/0010017].

[48] M. L. Mangano, M. Moretti, F. Piccinini, R. Pittau and A. D. Polosa, "ALPGEN, a generator for hard multiparton processes in hadronic collisions," JHEP 0307, 001 (2003) [arXiv:hep-ph/0206293].

[49] F. Maltoni and T. Stelzer, "MadEvent: Automatic event generation with MadGraph," JHEP 0302, 027 (2003) [arXiv:hep-ph/0208156].

T. Stelzer and W. F. Long, "Automatic generation of tree level helicity amplitudes," Comput. Phys. Commun. 81, 357 (1994) [arXiv:hep-ph/9401258].

H. Murayama, I. Watanabe and K. Hagiwara, "HELAS: HELicity amplitude subroutines for Feynman diagram evaluations," KEK-91-11

[50] E. Gerchtein and M. Paulini, "CDF Detector Simulation Framework and Performance," eConf C0303241, TUMT005 (2003) [arXiv:physics/0306031].

[51] R. Brun, F. Bruyant, M. Maire, A. C. McPherson and P. Zanarini, "Geant3," CERN$\mathrm{DD} / \mathrm{EE} / 84-1$

[52] G. Grindhammer, M. Rudowicz and S. Peters, "The Fast Simulation Of Electromagnetic And Hadronic Showers," Nucl. Instrum. Meth. A 290, 469 (1990). 
[53] F. A. Berends, H. Kuijf, B. Tausk and W. T. Giele, "On The Production Of A W And Jets At Hadron Colliders," Nucl. Phys. B 357, 32 (1991).

[54] V. M. Abazov et al. [D0 Collaboration], "Search for single top quark production in p anti-p collisions at s** $(1 / 2)=1.96-\mathrm{TeV}, "$ Phys. Lett. B 622, 265 (2005) [arXiv:hep$\mathrm{ex} / 0505063]$.

[55] D. Acosta et al. [CDF Collaboration], Phys. Rev. D 71, 012005 (2005) [arXiv:hep$\mathrm{ex} / 0410058]$.

[56] D. Acosta et al. [CDF Collaboration], "Measurement of the t anti-t production cross section in $\mathrm{p}$ anti-p collisions at $\mathrm{s}^{* *}(1 / 2)=1.96-\mathrm{TeV}$ using lepton + jets events with secondary vertex b-tagging," Phys. Rev. D 71, 052003 (2005) [arXiv:hep-ex/0410041].

[57] C. Hays et al., "The COT Pattern Recognition Algorithm and Offline Code", CDF Internal Note 6992 (April 2004).

[58] F. Abe et al. [CDF Collaboration], "The Topology of three jet events in anti-p p collisions at $\mathrm{S}^{* *}(1 / 2)=1.8-\mathrm{TeV}, "$ Phys. Rev. D 45, 1448 (1992).

[59] A. Bhatti et al., "Determination of the Jet Energy Scale at the Collider Detector at Fermilab," FERMILAB-PUB-05-470, arXiv:hep-ex/0510047 (2005). Submitted to Nucl. Instrum. Meth. A.

[60] S. Rappoccio, "Measurement of the $t \bar{t}$ Production Cross Section in $p \bar{p}$ Collisions at $\sqrt{s}=1.96$ TeV," Ph.D. Thesis, Harvard University (2005). Available as Public CDF Note 7894 and from http://www-cdf.fnal.gov/.

[61] C. Hill, J. Incandela, and C. Mills, "Electron Identification in Offline Release 5.3", CDF Internal Note 7309.

[62] V. Martin, "High-Pt Muon ID Cuts and Efficiencies for use with 5.3.1 Data and 5.3.3 MC", CDF Internal Note 7367 (March 2005).

[63] K. Bloom et al., "High pT Lepton ID Efficiency Scale Factor Studies in 4.11.1", CDF Internal Note 6858 (May 2004).

[64] H. Bachacou et al., "Preliminary Background Estimate of the SECVTX-tagged Lepton + Jets Sample", CDF Internal Note 7562 (March 2005). 
[65] A. Gibson, et al., "Parton-Jet Transfer Functions for the MEAT (Matrix Element Analysis Technique) $M_{t}$ Measurement", personal note (July 2004).

[66] F. Canelli, B. Mohr, and A. Gibson, "Jet Energy Transfer Functions in Lepton+Jets Events," CDF Internal Note 7715 (June 2005).

[67] J. Adelman, et al., "Generic Jet Scale Corrections for Run II," CDF Internal Note 7358 (February 2005).

[68] A. Bhatti, F. Canelli, L. Galtieri, B. Heinemann, "Out-of-Cone Corrections and their Systematic Uncertainties," CDF Internal Note 7449 (February 2005).

[69] J.-F. Arguin, P.K. Sinervo, "Revisiting the Top-Specific Jet Energy Corrections," CDF Internal Note 6404 (December 2003).

[70] M. F. Canelli, "Helicity of the W Boson in Single-Lepton ttbar events", Ph.D. Thesis, University of Rochester, Rochester, New York (2003). Also FERMILAB-THESIS-200322 .

[71] G. Mahlon, S. Parke, "Maximizing spin correlations in top quark pair production at the Tevatron", Phys.Lett.B. 411, 173-170 (1997).

[72] R.K. Ellis, W.J. Stirling, B.R. Weber, QCD and Collider Physics, Cambridge University Press (1996). Section 9.3.

[73] G. P. Lepage, "A New Algorithm for Adaptive Multidimensional Integration", Journal of Computational Physics 27, 192-203 (1978).

G. P. Lepage, "VEGAS: An Adaptive Multi-dimensional Integration Program", Cornell preprint CLNS 80-447, March 1980.

[74] M. Galassi, et al., GNU Scientific Library Reference Manual, 2nd Ed., ISBN 0954161734 .

[75] F. Canelli, et al., "Probability of W+Jets Events using Vecbos Matrix Element," CDF Note 7716, June 2005.

[76] K. Yorita, K. Kondo, "Update on the Measurement of Top Mass by DLM in the L+Jets", CDF Note 7642. 
[77] Jean-Francois Arguin, Pekka K. Sinervo, Si Xie, "Combined Measurement of the Top Quark Mass and the Jet Energy Scale in the Lepton+Jets Channel", CDF Note 7505. Section 7.1.

[78] Y.-K. Kim and U.-K. Yang, "Initial state gluon radiation studies on Drell-Yan data for top-pair production in hadron collider," CDF Note 6804, April 2004.

[79] S. Frixione and B. R. Webber, "Matching NLO QCD computations and parton shower simulations," JHEP 0206, 029 (2002) [arXiv:hep-ph/0204244].

[80] S. Frixione, P. Nason and B. R. Webber, "Matching NLO QCD and parton showers in heavy flavour production," JHEP 0308, 007 (2003) [arXiv:hep-ph/0305252].

[81] J. Pumplin, A. Belyaev, J. Huston, D. Stump and W. K. Tung, "Parton distributions and the strong coupling: CTEQ6AB PDFs," JHEP 0602, 032 (2006) [arXiv:hepph/0512167].

[82] A. D. Martin, R. G. Roberts, W. J. Stirling and R. S. Thorne, "Parton distributions and the LHC: W and Z production," Eur. Phys. J. C 14, 133 (2000) [arXiv:hepph/9907231]. 